\title{
UNIVERSITY OF SOUTHAMPTON
}

\section{Control Issues and \\ Low Back Pain}

\section{Lisa Carol Roberts}

A thesis submitted for the degree of Doctor of Philosophy

Faculty of Social Sciences Department of Social Work Studies

\author{
July 1999
}




\title{
UNIVERSITY OF SOUTHAMPTON
}

\author{
ABSTRACT \\ FACULTY OF SOCIAL SCIENCES \\ SOCIAL WORK STUDIES \\ Doctor of Philosophy \\ CONTROL ISSUES AND LOW BACK PAIN \\ by Lisa Carol Roberts
}

Acute low back pain is a commonly occurring symptom that can impact considerably upon a person's life. It is poorly defined, difficult to classify and challenging to measure. However, due to the high prevalence rates of the symptom, cited in the epidemiological literature, and the costs incurred by individuals, health services and society in general, it is vital that clients are encouraged to take more responsibility for their health.

This thesis is concerned with perceptions of control in people with acute low back pain. It is about how they respond to this symptom and the way it impacts upon their lives. The underpinning literature is drawn from both medical and social science research, as the work crosses faculty boundaries. Problems are addressed from a physiotherapeutic and social science perspective, as links are formed between the different disciplines.

From this literature, a significant theoretical development was the creation of a framework, which enabled the systematic review of existing outcome measures. A number of instruments, relevant to clients with acute low back pain, were reviewed using this framework, for the dimensions of control, function, pain and anxiety. The findings were used to inform the selection of outcome measures in this research.

Four studies were then undertaken, all linked to studying clients with acute low back pain. The first study was a survey of general practitioners in Southampton and the New Forest, which identified their strategies for managing clients with acute low back pain. The second study focused on clients' experiences and was a randomized controlled trial, which tested the effectiveness of an information leaflet, designed to encourage clients to take more responsibility for their own health.

The third study primarily focused on clients' perceptions of control over time, addressing the question of how these perceptions change during a one-year period. Finally, since recruitment of clients by their doctors was problematic throughout this research, a follow-up survey was undertaken with these health professionals to establish the reasons for this.

The results of these studies are used to discuss the importance of clients' perceptions of control and the implications for clinical practise. 


\section{Acknowledgements}

- Firstly I am deeply grateful to my supervisors - Judith Chapman, Frances Sheldon and Hazel Osborn for their encouragement, enthusiasm and guidance. Their constructive feedback has been greatly appreciated.

- Special thanks are due to my colleagues in the 'Back Home' team - Judith Chapman, Paul Little, Ted Cantrell, John Langridge and Ruth Pickering, from whom I have learned a great deal.

- Thanks are also due to all the clients who took part in this research and shared their experiences, and to the G.Ps who referred them. Without these individuals, this work would not have been possible.

- Throughout this research, the staff at the School of Occupational Therapy and Physiotherapy at the University of Southampton have been a great source of advice and inspiration which has been much appreciated, as has the support shown by colleagues in the Physiotherapy Department at Southampton General Hospital.

- Statistical advice was sought from Sue High in the Department of Social Statistics at the University of Southampton.

- The University of Southampton, N.H.S. Executive (Research and Development Directorate), St. Thomas' Association, Chartered Society of Physiotherapy and Arthritis and Rheumatism Council are gratefully acknowledged for their financial contributions. 


\section{Dedication}

To Paul:

With love and thanks for all his support, assistance and pearls of wisdom. 


\section{Contents}

Chapter one: Introduction............................................................................ 1

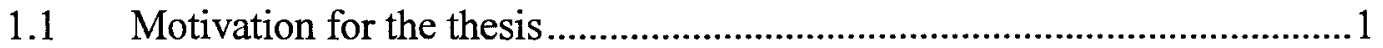

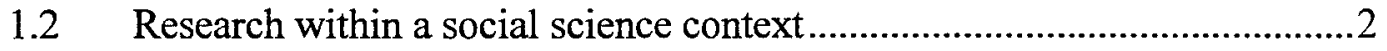

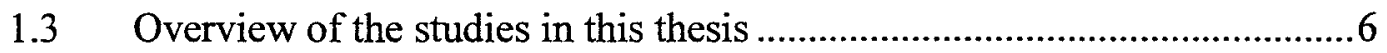

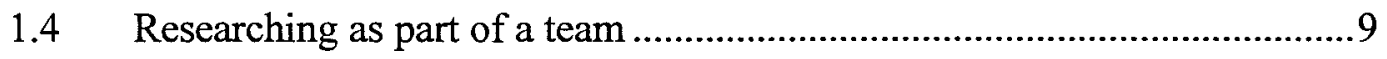

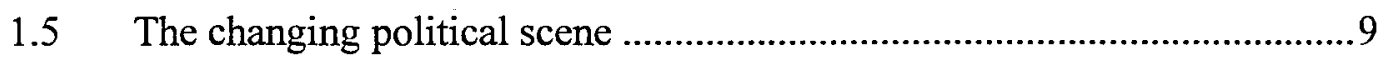

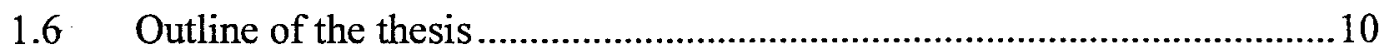

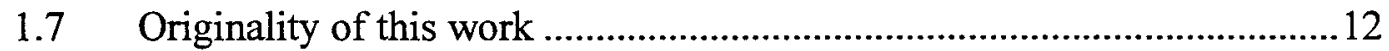

1.8 Publications accompanying this research ...............................................13

1.9 Summary of the chapter ........................................................................ 13

Chapter two: Review of the literature............................................... 14

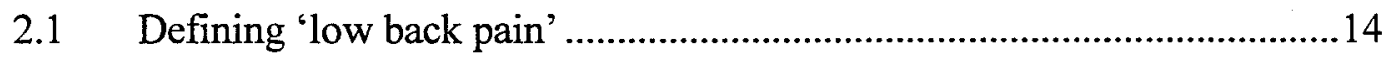

2.1.1 Site boundaries

2.1.2 Symptoms

2.1.3 Exclusion criteria

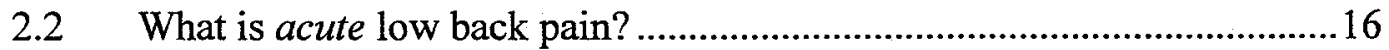

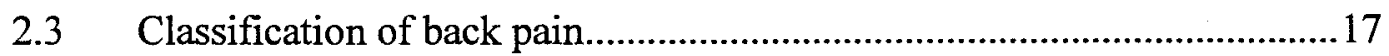

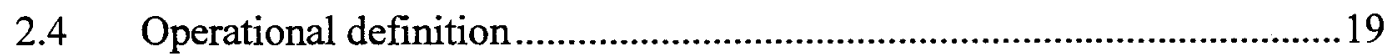

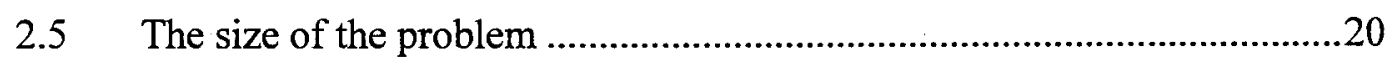

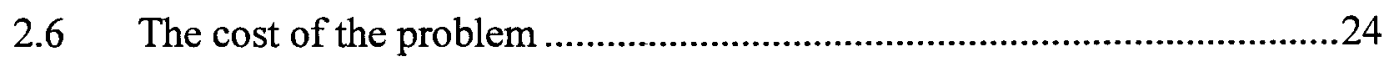

2.7 The natural history of low back pain ...................................................28

$2.8 \quad$ Recurrences of low back pain episodes ..................................................30

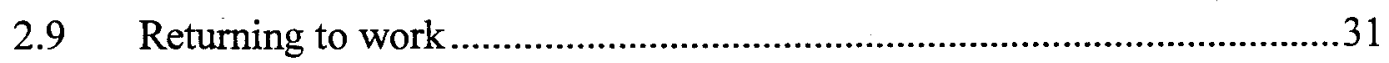

2.10 Conclusions from the back pain literature .............................................31

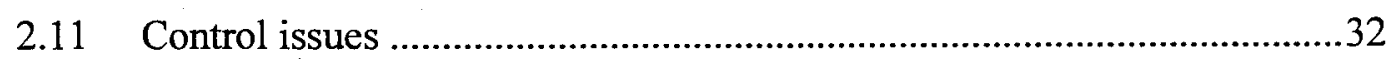

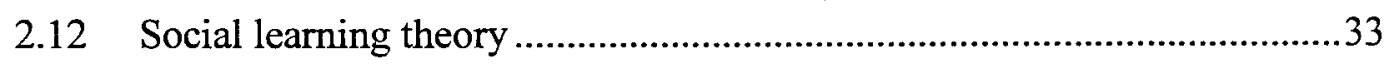

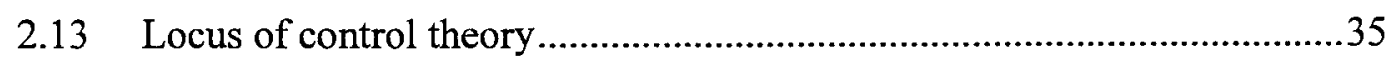

2.13.1 Self-efficacy

2.14 Variations in the locus of control construct.............................................42

2.14.1 Gender

2.14.2 Age

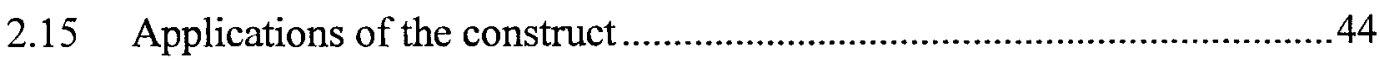

2.15.1 General health 
2.15.2 Information and health promotion

2.15.3 Pain

2.15.4 Chronicity of pain

2.15.5 Low back pain

2.15.6 Treatment for low back pain

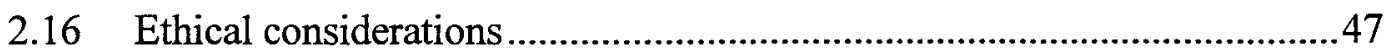

2.17 Limitations of the construct ..........................................................4

2.17.1 Limitations of the theory

2.17.2 Limitations of the measurement

2.17.3 Limitations of the research

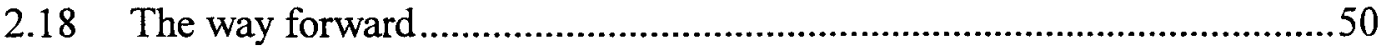

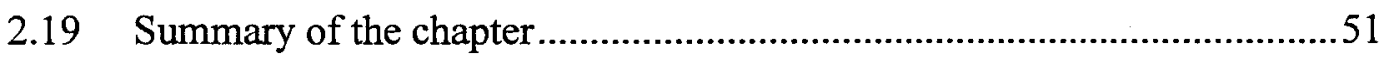

Chapter three: The G.P. questionnaire ................................................... 52

3.1 Acute back pain in general practice .......................................................52

3.1.1 Why do people consult their G.P.?

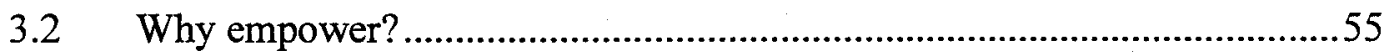

3.3 The pilot G.P. questionnaire ..............................................................57

3.3.1 Methods

3.3.2 The G.P. population

3.3.3 Procedures

3.3.4 Results of the G.P. pilot study

3.3.5 Reliability of the pilot questionnaire

3.3.6 Evaluation of the pilot questionnaire

3.4 Development of the final questionnaire 63

3.4.1 The question of danger signs

3.4.2 What evidence is there for danger signs (or 'red flags'?)

3.4.3 The question of $\mathrm{x}$-rays

3.4.4 What guidelines are there for referring clients for $\mathrm{x}$-rays?

3.4.5 Changes to the questionnaire following the pilot

3.4.6 Validity of the questionnaire

3.4.7 The final G.P. questionnaire

3.5 Results of the final G.P. questionnaire

3.5.1 Demographic details about the sample

3.5.2 How satisfied were G.Ps?

3.5.3 How do G.Ps assess?

3.6 How do G.Ps empower their clients? 
3.6.1 Advice about sleeping / mattresses

3.6.2 Advice about driving

3.6.3 Advice about specific back exercises

3.6.4 Advice about general fitness

3.6.5 Advice about sitting

3.6.6 Advice about lifting

3.6.7 Advice about gardening

3.6.8 The use of leaflets

3.6.9 What treatment modalities do G.Ps offer?

3.6.10 Why do G.Ps refer clients for x-rays?

3.6.11 Following clients up after consultation

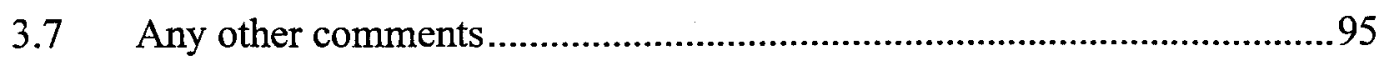

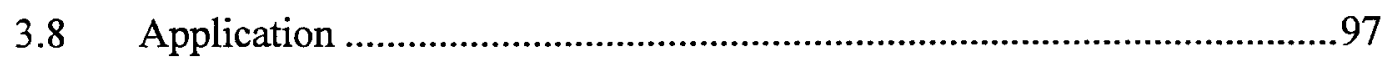

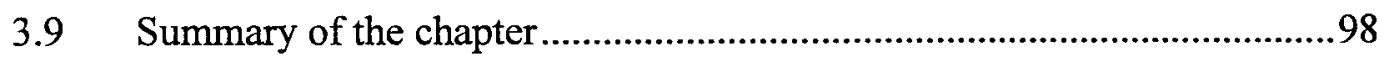

Chapter four: Measurement................................................................ 99

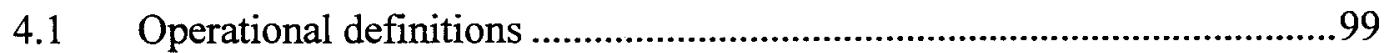

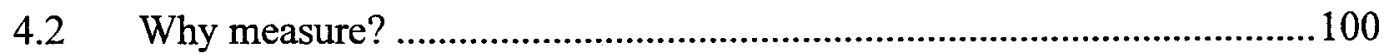

4.3 Are self-report measures appropriate? ................................................ 101

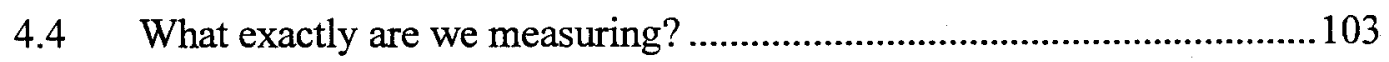

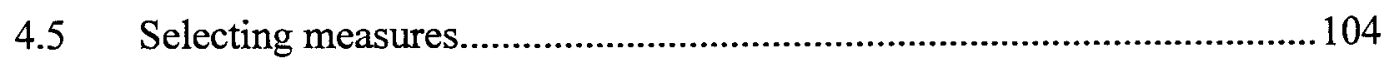

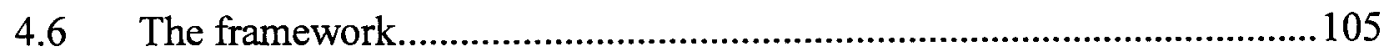

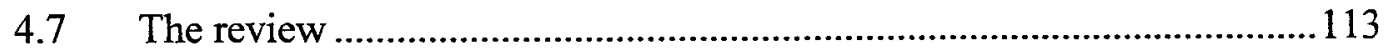

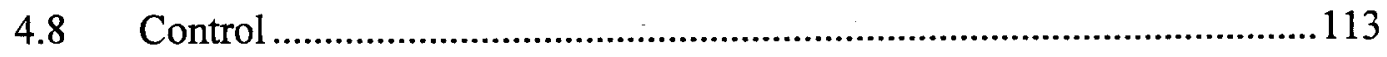

4.8.1 Rotter's I-E Locus of Control Scale

4.8.2 Multidimensional Health Locus of Control Scale

4.8.3 Perceived Control of Diabetes Scales

4.9 Function

4.9.1 Low Back Outcome Score

4.9.2 Waddell Disability Index

4.9.3 Oswestry Low Back Pain Disability Scale

4.9.4 Disability Questionnaire (Roland and Morris)

4.9.5 SF36

4.9.6 Sickness Impact Profile

4.9.7 Nottingham Health Profile

4.9.8 Aberdeen Low Back Pain Scale

4.10 Pain 
4.10.1 McGill Pain Questionnaire

4.10.2 Visual Analogue Scale

4.10.3 Numerical Rating Scale

4.10.4 Simple Descriptive Scale

4.11 Anxiety 131

4.11.1 State-Trait Anxiety Inventory

4.11.2 Short-form state scale of the State-Trait Anxiety Inventory

4.11.3 Hospital Anxiety and Depression Scale

4.11.4 Leeds Scales

4.11.5 Anxiety Thermometer

4.11.6 Multiple Affective Adjective Check List

4.12 Selecting measures for the 'Back Home' study.

4.12.1 Selection of control measures

4.12.2 Selection of function measure

4.12.3 Selection of pain measures

4.12.4 Selection of anxiety measure

4.12.5 Selection outcome measures in context

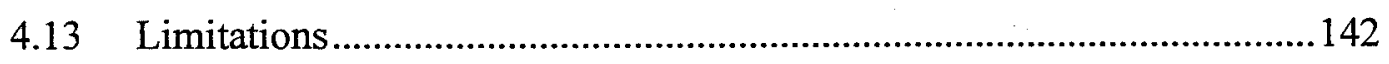

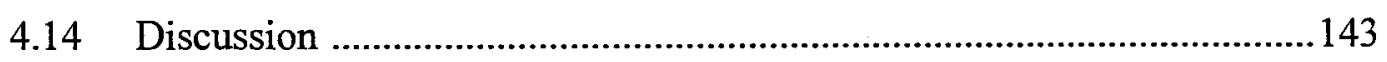

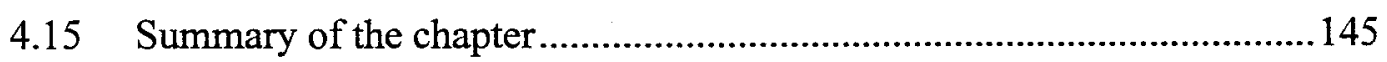

Chapter five: The 'Back Home' study........................................ 146

5.1 Why educate clients in health issues?................................................. 146

5.1.1 Ethical considerations

5.1.2 Health education issues

5.1.3 Barriers to client education

5.1.4 Why use a leaflet?

5.2 Aims of the 'Back Home' study .......................................................... 153

5.3 Development of the 'Back Home' leaflet .............................................. 154

5.3.1 Stage 1

5.3.2 Stage 2

5.3.3 Stage 3

5.3.4 Stage 4

5.3.5 Stage 5

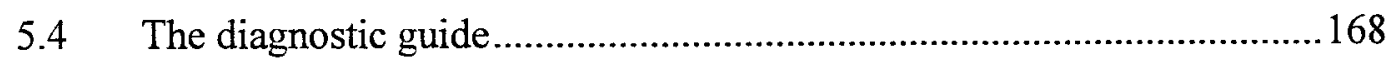

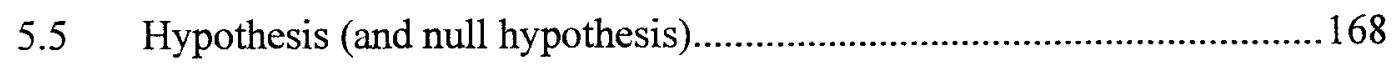

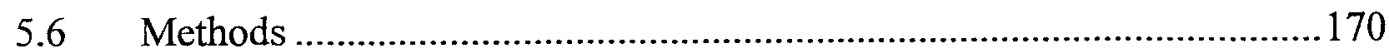


5.6.1 Study design

5.6.2 Ethical approval

5.6.3 GP recruitment

5.6.4 Randomization

5.6.5 Inclusion / exclusion criteria

5.6.6 The intervention

5.6.7 Client recruitment and consent

5.6.8 The sample size

5.6.9 Assessments

5.6.10 Administration

5.7 Outcome measures.

5.7.1 Knowledge measurement

5.7.2 Attitude measurement

5.7.3 Satisfaction measurement

5.7.4 Behaviour measurement

5.8 Client pilot study 180

5.8.1 Aims of the pilot study

5.8.2 The client population

5.8.3 Methods

5.8.4 Results of the client pilot study

5.9 Findings from the 'Back Home' study ............................................... 182

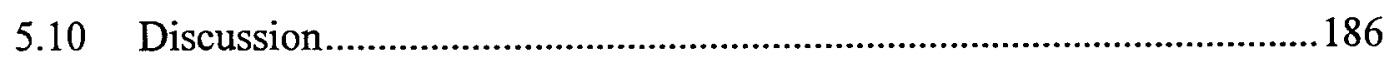

5.10.1 Sources of bias

5.10.2 Limitations of the study

5.11 Summary of the chapter.

Chapter six: Comparison of the M.H.L.C. (forms A \& C) ......... 193

6.1 Discovery of the condition-specific M.H.L.C. (form C) ......................... 193

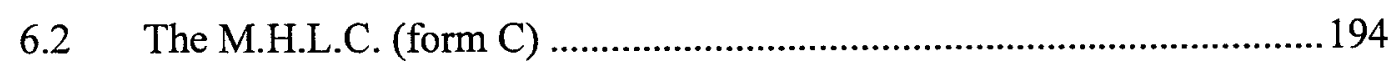

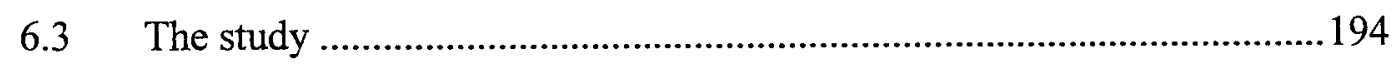

6.3.1 Results from assessment one

6.3.2 Results from assessment three

6.3.3 Results from assessment four

6.3.4 Results from assessment five

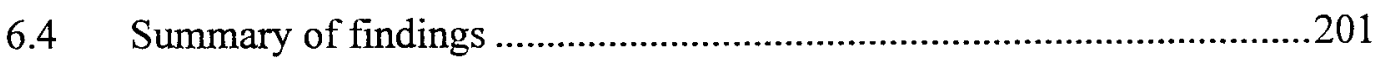

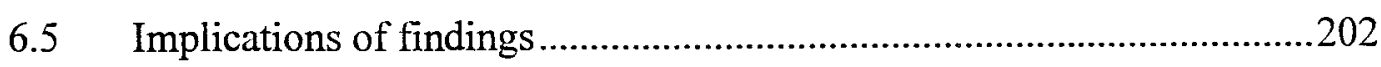

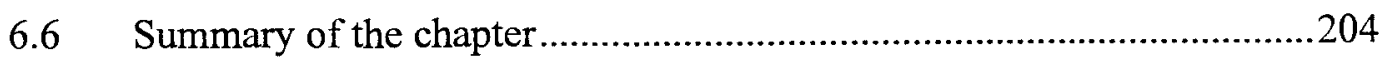




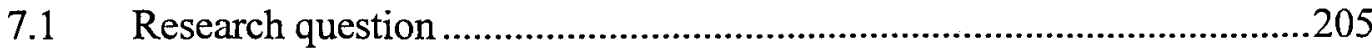

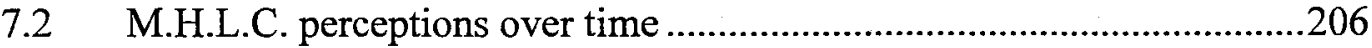

7.2.1 Influence of gender on M.H.L.C. perceptions

7.2.2 Influence of age on M.H.L.C. perceptions

7.2.3 Summary of M.H.L.C. perceptions over time

7.3 Perceived control perceptions over time...................................................215

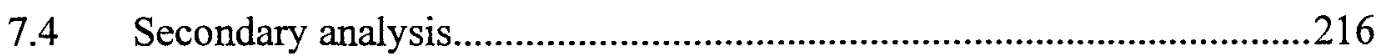

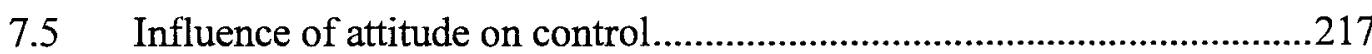

7.5.1 Attitude measurement

7.5.2 Analysis of attitude responses

7.5.3 Discussion of the results

7.5.4 Effect of attitude on perceptions of control

7.6 Influence of anxiety on control

7.6.1 Changes in clients' levels of anxiety over time

7.6.2 The effect of the 'Back Home' leaflet on clients' levels of anxiety

7.6.3 Association between clients' anxiety and perceptions of control

7.7 Satisfaction .226

7.7.1 Changes in clients' satisfaction levels over time

7.7.2 The effect of the 'Back Home' leaflet on clients' satisfaction

7.7.3 Association between clients' satisfaction and perceptions of control

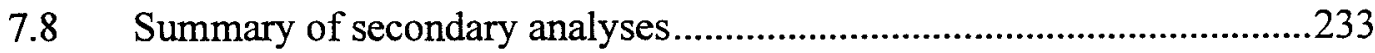

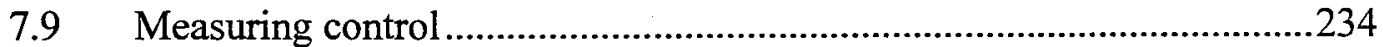

7.10 Summary of the chapter ..........................................................................235

Chapter eight: Following-up the G.Ps............................................. 236

8.1 The size of the recruitment problem .......................................................236

8.2 Following-up the original G.P. questionnaire.........................................237

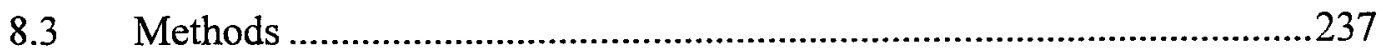

8.3.1 The G.P. follow-up questionnaire

8.3.2 The participating G.Ps

8.3.3 Scoring the questionnaire

8.4 Results of the questionnaire

8.4.1 Unprompted reasons for poor recruitment of clients

8.4.2 Prompted reasons for poor recruitment of clients

8.4.3 Any other comments

8.5 The implications of these findings for future research 
8.5.1 The composition of the research team

8.5.2 The use of incentives

8.6 Summary of the chapter.

Chapter nine: Overview........................................................................ 248

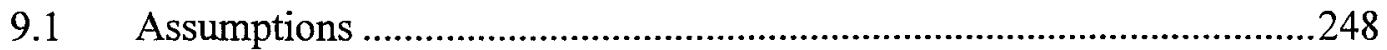

9.2 Key issues arising from the research .................................................250

9.2.1 Control issues

9.2.2 Outcome measures

9.2.3 Perceived control over health

9.3 Tensions.

9.3.1 The role of the researcher

9.3.2 Teamwork

9.3.3 Safety issues

9.3.4 Pragmatism versus idealism

9.3.5 Quantity of data collection

9.3.6 Negotiating an exit

9.3.7 Cross-faculty research

9.3.8 Issues of part-time study

9.4 Limitations

9.4.1 Generalizability

9.4.2 Level of randomization

9.4.3 Sample size

9.4.4 Inability to follow-up non-responders

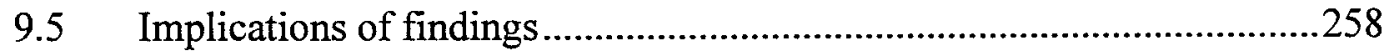

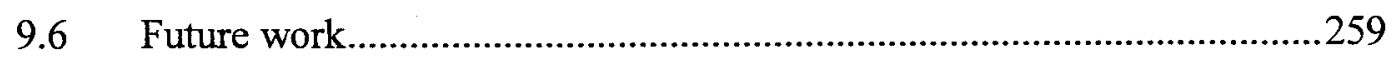

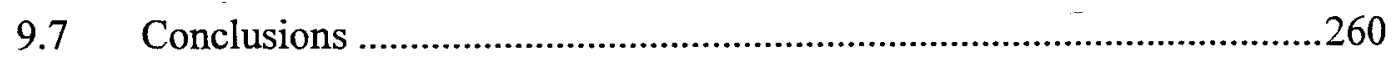

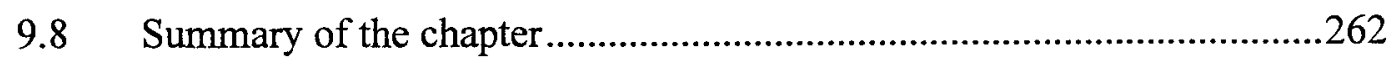

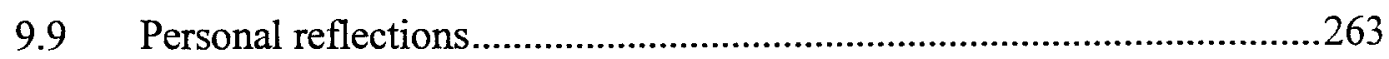

\section{References}

\section{Appendices}




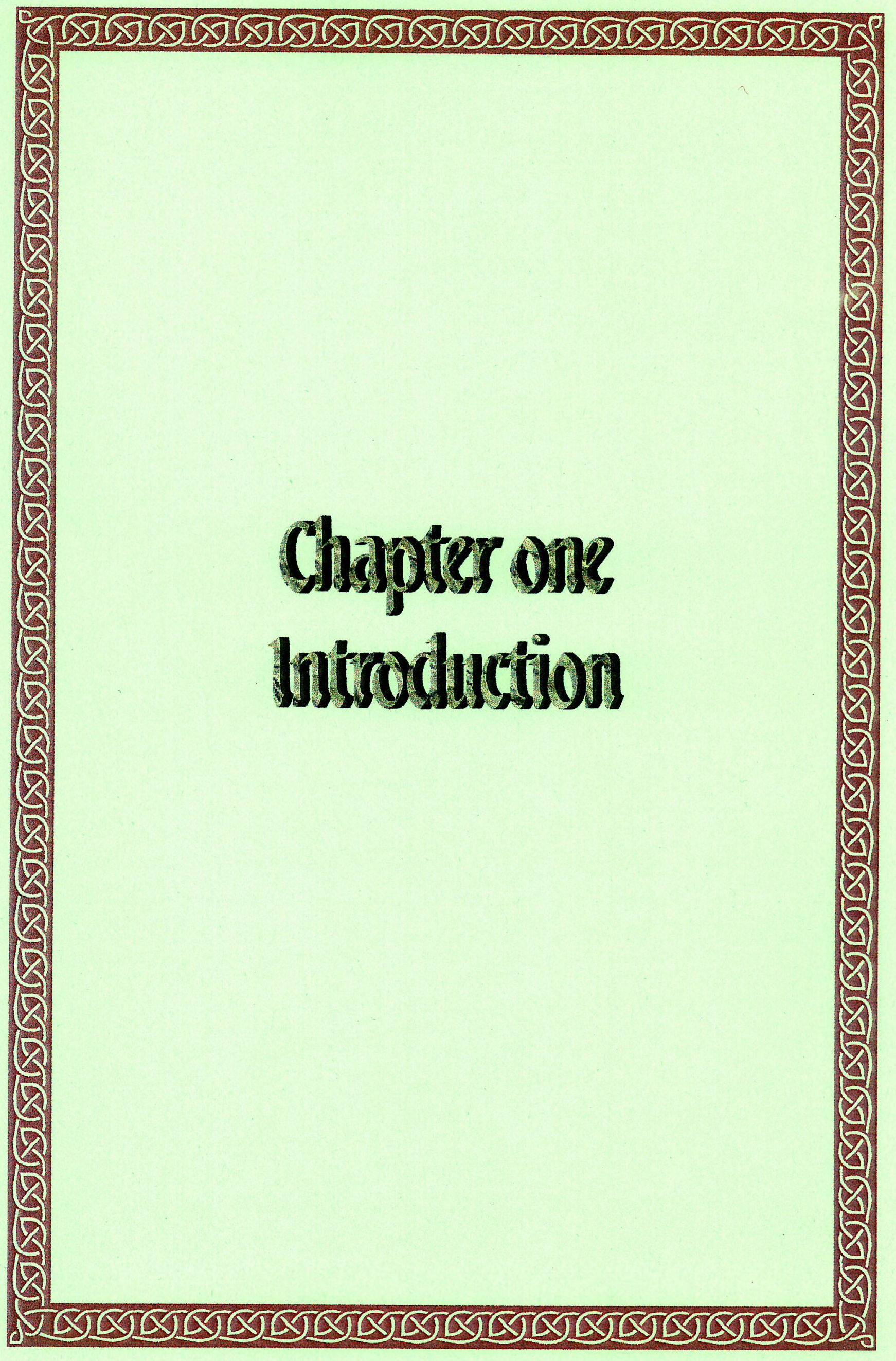




\section{Chapter one: Introduction}

Acute low back pain is a commonly occurring symptom that can impact considerably upon a person's life. It has both physical and psychosocial aspects, which could be influenced by a person's attitude towards their pain. This thesis is about how people respond to this common symptom and whether they perceive themselves able to influence their back pain episode.

In this introduction, the motivation for the research is discussed, its context, and the way the thesis is actually structured, including a brief summary of the content of each chapter. Accompanying the chapter (in appendices 1.1 and 1.2) there is a brief résumé of the major publications and presentations to date, that have directly resulted from this work.

\subsection{Motivation for the thesis}

In 1993, as a chartered physiotherapist treating musculoskeletal outpatients, it became apparent that clients' responses to treatment were not just influenced by their signs and symptoms, together with the abilities of the therapist - there was something more. Clinically it appeared that clients' perceptions of their symptoms and their attitude towards them, were important factors. At this stage, it was merely an observation.

Nowhere was this more apparent, than in our newly-established Backschool (a series of two early evening sessions offered as an adjunct to clients' treatment), which addressed simple biomechanics, ergonomics, advice and exercises, designed to help clients manage their symptoms themselves. Some participants at these sessions appeared highly motivated to take an active role in their rehabilitation - in others, this trend was either more subtle, or non-existent. What was interesting as a clinician, was to consider what difference (if any), these variations in attitudes might make to clients' recovery from their symptoms. Therefore, this mere observation was starting to have clinical significance, and the first stage was to evaluate clients' perceptions of the usefulness of the Backschool, and how they were using its content (or not).

Meanwhile, funding had been secured from the Research and Development Directorate (then, the Wessex Regional Health Authority), to investigate the effectiveness of a leaflet 
in the management of acute low back pain. This work provided an opportunity to explore perceptions of control in this client group, and therefore, it was possible to investigate these issues alongside the work on the client-information leaflet.

\subsection{Research within a social science context}

Before outlining the content of this thesis, it is necessary to consider the context in which the research has occurred.

Traditionally, owing to its close links with the medical profession, physiotherapy has adopted a 'strictly biomedical model'(1). This model was based on the principle that 'disease and disability arise from deviations in normal physiology and does not take into account psychology and human behaviour ${ }^{(1)}$. It is a reductionist model, which aims for specificity of diagnosis and management strategy.

The philosophy underpinning medical thinking is often described as a product of modernity: a notion which 'places man at the centre' and perceives him as a 'rational being $^{\prime(2)}$. The guiding principles of modernity can be thought of as a belief in progress, technical expertise, order and values that are universal ${ }^{(3)}$, and Howe defines the characteristics of the modern age as discipline and progress, order and change ${ }^{(4)}$. Modern thinkers have been described as quoting Bacon - knowledge is power ${ }^{(2)}$ - and thus gleaning coherent and integrated knowledge is fundamental to this way of thinking.

According to Biggs, medicine and nursing have drawn heavily on technical and scientific knowledge "to justify their expert status ${ }^{\text {(3) }}$ and this can be applied to physiotherapy, a profession closely allied to medicine, which is grounded in technical knowledge, and seeks an emerging scientific evidence-base.

More recently, the importance of psychological processes has been recognized in medicine (and physiotherapy), and the biomedical model has been superceded by a biopsychosocial model of medicine, which, according to Klaber Moffett, 'puts equal emphasis on biological, psychological and environmental factors in the interpretation of illness and pain ${ }^{(1)}$. This approach is more liberating than the former biomedical model, although it is also more challenging to research, as it is more complex. 
Specific to back pain, Waddell has proposed a biopsychosocial model for the clinical presentation of assessment of low back pain and disability ${ }^{(5)}$, which considered pain within the context of clients' attitudes and beliefs, psychological distress, illness behaviour and the social environment ${ }^{(5)}$. He advocates treating low back pain as a human illness (defined as the total experience of disease as perceived by the patient), rather than as a disease (or active pathology) $)^{(6,7)}$. It is important that assessments of clients with low back pain consider its holistic nature - they are not considering an anatomical structure such as a disc or zygapophyseal joint, nor a pathological process such as a fracture or degenerate spine, but a person experiencing low back pain.

Waddell's biopsychosocial model has been represented diagrammatically, as shown in figure 1.1. Although this model has its limitations, e.g. it has been criticized for suggesting that back pain starts as a physical problem and all the psychosocial changes develop secondarily ${ }^{(\text {Reilly in 5) }}$, it is useful as it integrates physical findings, psychological and social issues.

\section{Figure 1.1: A biopsychosocial model of low back disability ${ }^{(5)}$}

Reproduced with kind permission from Her Majesty's Stationery Office

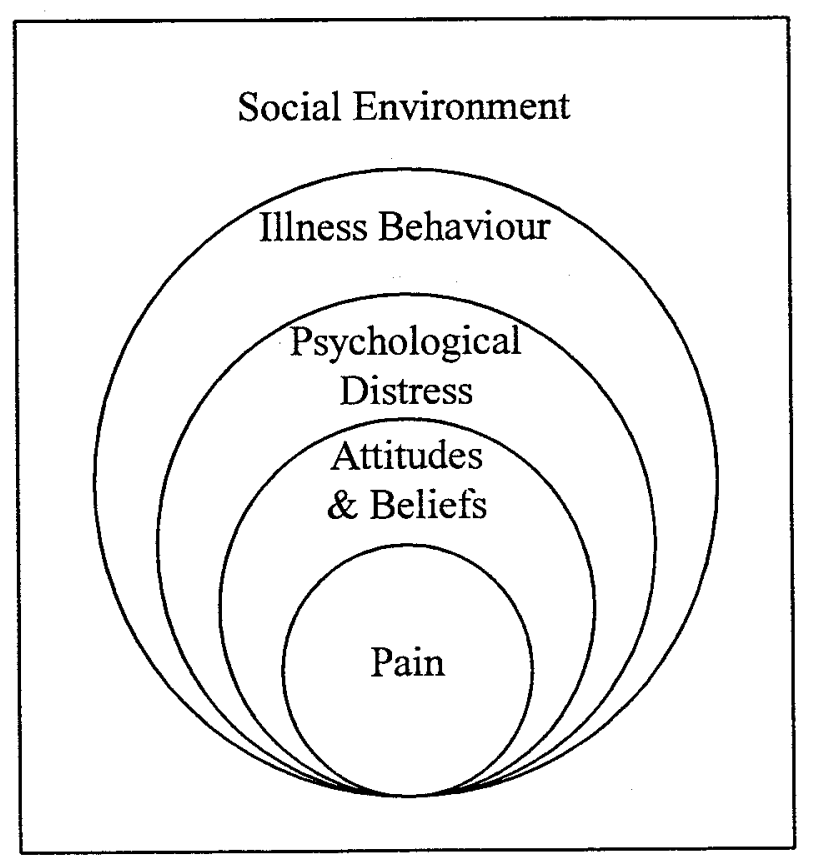


Waddell suggests that patients and health professionals tend to see back pain 'as if through a series of filters'(5), and it is vital that the whole picture is considered, not just the filtered version, when trying to study human response to back pain.

Attempting to grasp the complexities of back pain from a physiotherapy perspective (which incorporated the biopsychosocial model), although helpful, had resulted in a limited understanding of the subject. Many questions remained unanswered, such as whether clients' perceptions of their signs and symptoms affected their treatment progress?

Therefore, to try and gain a wider understanding of the human response to back pain, it seemed appropriate to turn to the social sciences for a fresh approach to this issue. This did not appear to have been previously done, specifically for acute low back pain, linking physiotherapy and social science.

Social science has been defined as '... the study of human behaviour by scientific $\operatorname{methods}^{(8)}$, and while the natural sciences seek to understand nature, the social sciences aim to explain people, society and the human condition ${ }^{(4)}$.

According to Elmer Barnes, the social sciences were 'created by the industrial revolution' which he described as the greatest transformation in the history of humanity ${ }^{(8)}$. Perhaps surprisingly, the individual disciplines that are sited within social sciences are not consistent, for example history is sometimes classified as a social science, whereas at other times it is considered part of the 'Humanities'(8). This suggests that the organization of the social sciences is 'largely a matter of convenience ... rather than a reflection of intrinsic differences in subject matter ${ }^{(8)}$.

Within the social sciences there were two disciplines which were particularly pertinent to studying control issues and back pain: social work studies and health psychology.

Howe claims that it was 'within the nineteenth century's growing ideas about the relationship of the individual to society that social work formed', and it has been interested in explaining and changing society ${ }^{(4)}$. He advocates that the 'essential' character of social work formed under modernity's identification of 'universal standards of truth, goodness and beauty applied to the self and society ${ }^{\text {(4) }}$ and it has been closely 
identified with notions of university and equity ${ }^{(3)}$. However, this discipline has progressed and may now be perceived as 'post-modern'.

According to one dictionary definition, postmodernism denotes a movement 'reacting against modern tendencies, especially by drawing attention to former conventions ${ }^{\text {(9) }}$. The term post modern indicates that it comes into existence after modernity ${ }^{(2)}$, but there is no agreement about when the world actually entered its postmodern phase $\mathrm{e}^{(4)}$. Nietzsche's writings in the last century, which rejected the idea that any one system of belief could ever reveal the whole truth, are sometimes cited as the beginnings of a postmodern world $^{(4)}$. However, some authors debate whether postmodernity is actually a break with modernity, or merely its continuation ${ }^{(2)}$.

Postmodern thought is characterized by a loss of belief in an objective world ${ }^{(2)}$, accepting the unpredictability that is inherent in the world ${ }^{(10)}$. It contrasts with modernity, which attempted to legislate what is the truth, whereas according to Bauman, in the postmodern world, the truth can only be interpreted ${ }^{(11)}$. Kvale purports that postmodern thought goes beyond the cognitive and scientific domain to also include the 'ethical and aesthetic domains of life in reason'(2). This is useful for studying human behaviour, where little can be truly known, whereas much can be interpreted.

Postmodernism marks a shift towards a more fluid state of affairs ${ }^{(3)}$ than was understood in the modern world. The hub of postmodern life strategy is not identity building, but the avoidance of being fixed ${ }^{(11)}$. As Bauman says, modernity was built in steel, whereas postmodernity is built in biodegradable plastic ${ }^{(11)}$.

The role of the social scientist in the postmodern world is, according to Howe, 'not to cure, control and legislate according to alleged universal standards but to interpret and understand one world and present it to another ${ }^{(4)}$. He goes further to say that if being in a critical, self-reflexive, de-centred and deconstructive state of mind captures the mood of postmodernity, then social work might also be said to be in a postmodern $\operatorname{mood}^{(4)}$.

This contrasts with the discipline of psychology, which has been described as a "project of modernity ${ }^{\prime(2)}$. Psychology does not sit comfortably with the fluidity and unpredictability of postmodernity and indeed, Kvale has described 'postmodern psychology' as a contradiction in terms ${ }^{(2)}$. 
Like psychology, physiotherapy is perhaps more akin to the modernists view of the world than the postmodern view. It is a pragmatic, clinical profession, striving for an underpinning scientific evidence-base, which requires rigor and reliability, rather than unpredictability. Therefore, in bridging the gap between physiotherapy and social science, although initially, it felt more 'comfortable' to side with the discipline of psychology in wanting to gain a wider understanding of the human response to back pain, it was recognized that this was more likely to be achieved by taking a postmodern stance, which could be pursued through the discipline of social work studies. Here, the experience of studying human behaviour could usefully complement physiotherapy practice and research. This new approach was somewhat daunting.

Therefore, this work deliberately spans professional and faculty boundaries, which has been reflected by joint supervision (and examination) across both physiotherapy and social science disciplines. An example of this cross-linking can be seen in the literature review (in chapter two), where much of the epidemiology of acute low back pain is summarized from the 'medical' literature, whereas the theory underpinning the control concepts arises from the social sciences.

Bridging such gaps between faculties is in keeping with current thinking about 'good practice', which, according to the Open University, involves flexibility of role and roleboundaries and working across organizational structures ${ }^{(10)}$. This needs sophisticated interpersonal skills ${ }^{(10)}$ and it is here that social work and physiotherapy can really complement each other, especially since at the heart of both professions, is involvement with people.

\subsection{Overview of the studies in this thesis}

Having purported that this research crosses professional and faculty boundaries, this section outlines the four main studies in this thesis. (The specific content of each chapter is addressed later, in section 1.6.)

Overall, the methods chosen are essentially quantitative, arising from the medical perspective, which, as already discussed, is underpinned by a philosophy of modernity. It is futile to debate which is preferable - qualitative or quantitative approaches, since as 
Gale says, both can be seen as appropriate at different stages in the research process ${ }^{(12)}$. What is particularly pertinent, is how the methods are executed.

It can be seen in chapter two that the existing knowledge base on psychosocial issues is limited for people with acute low back pain. Therefore, quantitative methods can be employed to provide relatively simple information covering a wide focus on this topic, which could prove both a useful overview and foundation upon which to base further research.

Using a systematic, quantitative approach, such as questionnaires, ensures that identical material is considered by all clients and the possibility of omitting topics is limited, as the questionnaires are completed in the presence of the researcher.

Although such methods do enable copious data to be collected, data management is relatively straightforward and secondary analyses can be performed. In this work for example, it was possible to explore the influence of attitude, anxiety and satisfaction on aspects of control, discussed in chapter seven.

It is also important when selecting methods to consider the effect of the researcher on the data collected. According to Hicks, the researcher in quantitative research is deemed 'detached and objective', whereas in more qualitative research, they become more 'enmeshed and integral with the people being studied' ${ }^{\text {'(13) }}$. This is an over-simplification, since in this research, by going into peoples' homes, interacting with them and overseeing the data collection, it was impossible to be 'detached'. This tension is discussed in section 9.3.1. In addition, because self-report outcome measures were predominantly used in this research, this notion of objectivity must be questionable.

Despite the obvious advantages of choosing quantitative methods, there are several disadvantages. Firstly the data collected are usually more superficial than data gained from qualitative approaches, where the researcher has more freedom to discuss particular issues in more depth, in response to what clients say. In addition, a standardized questionnaire assumes that all participants can read and write and is difficult to adapt to different client abilities. This is a disadvantage when compared with an interview for example, where virtually all participants can communicate verbally and thus express themselves according to their own ability. 
Apart from these technical issues, completing multiple questionnaires can be timeconsuming, tedious and repetitive, which must be considered in determining methods of data collection.

On balance, due to the limited literature published on control issues in people with acute low back pain and the constraints of working alongside a funded research project, quantitative methods were chosen for this work, despite their limitations. In addition, more qualitative aspects, such as semi-structured interviews and field notes were used to give the data depth and they emerge frequently throughout the forthcoming chapters.

In this thesis, the first study, described a survey of all G.Ps in Southampton and the New Forest to identify their current strategies for managing clients with acute low back pain. It was clear from this work that G.Ps potentially had considerable opportunity to influence whether (or not) clients feel empowered by the actions or advice of their G.P., to manage their back pain. This influence potentially arose from assessment practices, investigations, advice, treatment, or even negotiating an exit from the consultation.

Having considered the experience of back pain from the G.Ps' perspective, the second study focused on the clients' experiences. This work necessitated developing an information leaflet (called 'Back Home') which was designed to encourage clients to take more responsibility for their own health. The effectiveness of this leaflet was evaluated in a randomized controlled trial, where clients were followed-up, by home visit, within two days of their initial visit to the G.P., at two weeks and at three months.

Thirdly, in order to specifically address clients' perceptions of control in this population, further home visits were undertaken at six and twelve months, which created an opportunity to study changes in clients' perceptions of control over time. In total, 272 home visits were completed by the author (plus two postal and one assessment of a client in the physiotherapy department), between June 1994 and December 1997.

As discussed in chapter five, the recruitment of clients into the study was considerably slower than estimates from earlier pilot work. Therefore, in order to investigate this mismatch between G.Ps' estimates of potentially suitable clients and the reality of the number of clients recruited into this study, a further survey was undertaken with G.Ps. The findings of this work are reported, together with a discussion of the implications for funding future studies that necessitate recruiting clients from G.Ps' routine surgeries. 
In addition to these four main studies, the thesis includes a study comparing a conditionspecific outcome measure (the Multidimensional Health Locus of Control - form C) ${ }^{(14)}$, with its more general version (form A), used to measure control issues in clients with acute low back pain.

\subsection{Researching as part of a team}

The G.P. survey, the randomized controlled trial evaluating the 'Back Home' leaflet and G.P. follow-up study were planned by the research team which, in addition to the author, comprised: a lecturer in physiotherapy; consultant rheumatologist; general practitioner; manager of physiotherapy services; and statistician.

Early in the research process, team members interests were established, which enabled 'ownership' of writing specific aspects of the research. My specific contributions were: the measurement of acute low back pain (discussed in chapter four); the control issues (addressed in chapters six and seven), which included the comparison study; and the theory underpinning danger signs for acute low back pain (described in chapter three).

The process of actually doing research as part of a multi-professional team is addressed in section 9.3.2, which also includes some reflective thoughts on the whole experience of researching clients with acute low back pain.

\subsection{The changing political scene}

In putting the work in this thesis into context, it is important to address changes in the political scene regarding back pain, which occurred during the course of the research.

In the final stages of this research, the literature includes many different models and clinical guidelines for both physical and psychosocial factors. However, at the onset of this work, this knowledge was not available, and therefore much work had to be done to determine clinical standards for managing clients with acute low back pain. At that time, only one set of national guidelines was published, which were the findings of the Quebec Task Force on Spinal Disorders, from $1987^{(15)}$. The management of low back pain in the United Kingdom was in a state of disarray. Aware of the spiralling reported disability 
and cost of this symptom, the Government recognized the need to research back pain in the Health of the Nation document ${ }^{(16)}$, before any national targets could be set.

In the midst of this disarray (in 1994), the Agency for Health Care Policy Research (A.H.C.P.R.) published the American clinical guidelines for acute low back pain ${ }^{(5,17)}$. These guidelines contained 'the most thorough review ever performed of the scientific evidence on back pain up to $1992^{\prime(5)}$.

The first U.K. guidelines were developed by the Clinical Standards Advisory Group (C.S.A.G.), and were published in December $1994^{(5,18)}$, with the purpose of reviewing the scientific evidence on effective treatments for acute low back pain ${ }^{(5)}$. The second edition of the U.K. guidelines was developed by the Royal College of General Practitioners, and was published in September $1996^{(5,19)}$. Since then, the New Zealand Acute Low Back Pain Guide has been written ${ }^{(20)}$, which places stronger emphasis on psychosocial factors and the prevention of chronic pain and disability ${ }^{(5)}$, something we had already recognized.

In addition to the development of clinical guidelines, changes in health service organization (such as G.P. fund-holding), pressure on orthopaedic waiting lists and demand for complementary therapy on the N.H.S. 'led to questions about the effective delivery for services for patients with low back pain'(21).

Thus, there is no better time for researching the effectiveness of interventions in clients experiencing back pain. In addition, physiotherapists are also moving into primary care in an extended practitioner role, and are now often physically located within a G.P. practice. Therefore, it is paramount that clinical practice is evidence-based and will stand the rigors of inter-professional and academic scrutiny.

As the studies in this thesis draw to a close, there is even greater recognition of the importance of the initial consultation for clients with acute low back pain, and as yet, the impact of the U.K. clinical guidelines has not been reported.

\subsection{Outline of the thesis}

Having discussed the motivation of this thesis, and the research in context, a brief summary of each chapter is included as an overview. Since the research comprises four 
separate studies, the thesis has been structured so that each study forms a distinct chapter. (It was considered that one chapter covering the methods of all the studies for example, would be unwieldy.) Therefore the chapters are as follows:

Chapter two considers the problem of back pain - its definition, classification, epidemiology, cost, natural history and its recurrence. As already mentioned, this material is derived mainly from the 'medical' literature. Then, issues of control are addressed (primarily from the social sciences literature), including the locus of control construct, perceived control, self-efficacy and the influence of gender and age on the locus of control concept. Limitations of the theory, its measurement and its application to research are highlighted.

Chapter three describes a survey of G.Ps in Southampton and the New Forest, which aimed to identify their current treatment strategies for the management of acute low back pain. This work was undertaken prior to the release of the C.S.A.G. report on the management of back pain ${ }^{(18)}$, and the clinical guidelines produced by the Royal College of General Practitioners ${ }^{(19)}$, and was therefore at a time when G.Ps' management strategies were often developed individually. The focus of the analysis is on six aspects of empowerment i.e. opportunities that G.Ps have to influence clients (by their actions and advice).

Chapter four addresses the issue of measuring acute low back pain and it proposes a framework, which would allow the systematic review of existing outcome measures. Once this framework is outlined, a critical review of instruments for measuring control, function, pain and anxiety is presented, which includes the rationale for the selection of outcome measures chosen in this research.

Chapter five includes the detail of the development (in five stages) of the 'Back Home' leaflet, and its subsequent evaluation in a randomized controlled trial. Participating clients were followed-up by home visit for three months, and the analysis primarily focuses on changes in clients' knowledge, attitude, behaviour and function.

Chapter six describes a simple comparison study of the 'new', condition-specific outcome measure - the Multidimensional Health Locus of Control (form C) ${ }^{(14)}$, with the more general, established, form $\mathrm{A}^{(22)}$. 
Chapter seven then uses this 'new' instrument to explore clients' perceptions of control. The analysis primarily focuses on changes in perceptions of control over a period of one year, and then explores the influence of the 'Back Home' leaflet on these control perceptions, and age and gender influences. The chapter also includes a broader analysis, which investigates the influence of attitude, anxiety and satisfaction on clients' perceptions of control.

Chapter eight describes a small, questionnaire survey, which follows up the poor recruitment of clients into the 'Back Home' study, and establishes G.Ps' perceptions of why this occurred. The implications of these findings are then discussed, in relation to funding issues for future research in primary care, using similar methods.

Chapter nine finally draws together the research strands discussed in the preceding chapters, and reflects on the tensions that occurred within the research process, as well as the limitations of the studies undertaken. The chapter considers the implications of the research findings and summarizes the conclusions from the four studies outlined in chapters three, five, seven and eight, suggesting ways of further developing these research ideas.

The references for the whole thesis are included at the end of the work, arranged by chapter.

\subsection{Originality of this work}

Having considered the overall structure of this thesis, it is important to consider its clinical relevance.

As far as could be determined, this was the first prospective study of clients perceptions of control in a population of people with acute low back pain, followed over a one year period. It could be considered part of an era of medical research involving clients with acute low back pain in which psychosocial factors are considered alongside more traditional biomedical factors.

In addition, at the onset of this work, there was no reported comparison study of the condition-specific M.H.L.C. (form C) with its earlier versions for clients with acute low 
back pain, and this needed to be rectified if the instrument was to be used as an outcome measure in this research, and subsequent low back pain studies.

Although many journals and books existed which summarized outcome measures, this was the first such critique of instruments for use in a study of clients with acute low back pain. This perhaps is the single, most important contribution to the body of knowledge, of this piece of research. Had such a summary of outcome measures been available at the onset of this work, this would have vastly simplified the selection of appropriate instruments, by highlighting the strengths and weaknesses of each tool. Publication of this work is a priority on completion of this degree.

\subsection{Publications accompanying this research}

To date, a number of articles and reports have been published. These are listed in appendix 1.1. In addition, aspects of this research have been presented at five national or international conferences. Details of these are presented in appendix 1.2.

\subsection{Summary of the chapter}

- The motivation for this thesis has a clinical grounding, initially stemming from clients' attitudes, previously demonstrated in a Backschool.

- In ascertaining a wider understanding of the human response to back pain, it was necessary to cross professional and faculty boundaries, thus approaching the problem of acute low back pain from both a physiotherapy and social science perspective.

- As far as can be determined, this is the first prospective study of clients perceptions of control in a population of people with acute low back pain, followed over a one year period. 


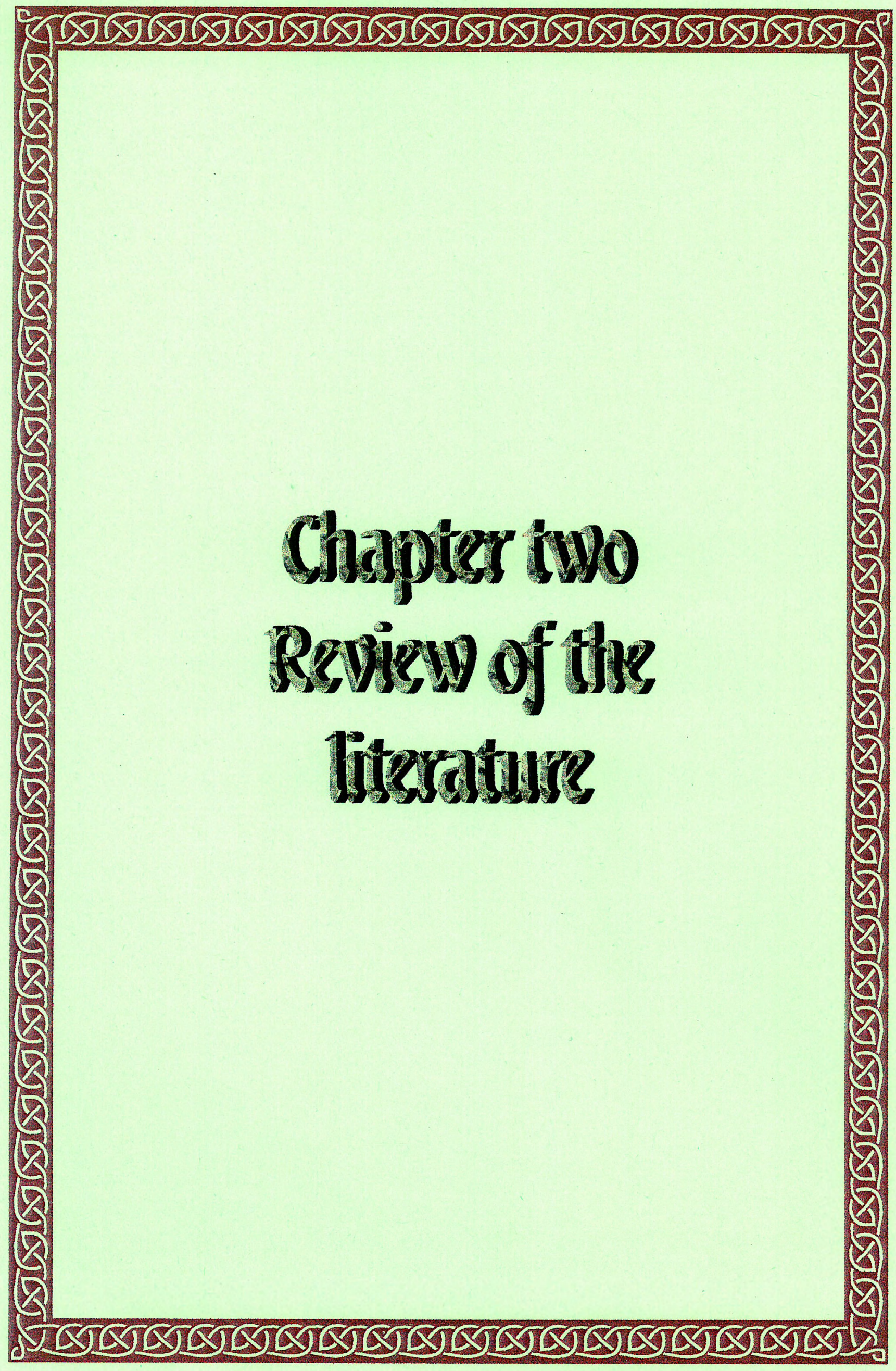




\section{Chapter Two: Review of the Literature}

This chapter reviews the literature published on acute low back pain and control issues. The information about back pain, including its definition, epidemiology, natural history and cost to society is taken primarily from the 'medical' and public health literature. By contrast, the literature surrounding control issues, including illness beliefs, the locus of control construct and its applications is taken mainly from the psychological and psychosocial literature.

It is perhaps surprising that from the two vast fields of knowledge (low back pain and locus of control), the reported area of common ground is minimal. The chapter draws together these two fields and outlines the need for closer integration of medical and psychosocial research, particularly in professions such as physiotherapy, where the whole client must be considered, not just their medical or psychosocial well-being.

\subsection{Defining 'low back pain'}

Low back pain is a symptom - it is not a disease entity ${ }^{(1)}$, and it is reported to be experienced by $50-80 \%$ of us at some time in our lives ${ }^{(1,2,3,4,5)}$. The reason that this reported lifetime experience is so variable is due to differences in the demographic variables, definitions and methods of the samples studied. This is further discussed in section 2.5 .

Perhaps surprisingly, there is no universal, standardized definition of what constitutes 'low back pain' and some authors such as Walsh, do not seem convinced that this can be readily achieved, arguing that any definition 'must be arbitrary', in view of our uncertainty of the pathogenesis ${ }^{(1)}$.

Despite this less-than-optimistic view, in Scandinavia, Kuorinka et al. have attempted to standardize the definition of 'low back trouble' but their definition has not been widely adopted in the literature. They define 'low back trouble' as an 'ache, pain or discomfort in the shaded area [of a diagram] whether or not it extends from there to one or both legs (sciatica) $^{(6)}$. However, alongside the diagram, Kuorinka et al. state that 'limits are not sharply defined ${ }^{(6)}$, which is clearly unacceptable since in any standardization, the 
anatomical limits must be clear and precise. Presumably this lack of precision has contributed to the Scandinavian definition not being widely adopted.

At the very least, any definition of low back pain should include the anatomical site boundaries, symptoms under review and any exclusion criteria. These are now considered.

\subsubsection{Site boundaries}

The majority of researchers investigating aspects of back pain fail to specify the anatomical boundaries of the area they are studying, thus rendering it impossible to make detailed comparisons between many studies.

When anatomical boundaries are stated, considerable variation exists. Perhaps the most commonly cited low back pain area is from the lowest ribs superiorly to the gluteal folds inferiorly $^{(7,8)}$, although by comparison, Bergquist-Ullman defines the site boundaries as 'the lower lumbar area or in the paravertebral region [as illustrated in a diagram] $]^{\text {(9) }}$ whilst Magora outlines an area below the $10^{\text {th }}$ thoracic vertebra ${ }^{(10)}$. Less specifically, Valkenburg and Haanen argue that the lumbar region cannot be studied in isolation from other parts of the spinal column, and that in obtaining 'a comprehensive view', they include the shoulders ${ }^{(11)}$.

Meanwhile, some authorities such as the Quebec Task Force for Spinal Disorders (a team comprising clinicians, health professionals and methodologists), have attempted to overcome arbitrary site boundaries by including the whole area, categorizing 'spinal disorders' as musculoskeletal complaints relating to the entire spine (excluding 'multiple injuries in a given accident' $)^{(12)}$. Therefore this team do not attempt to define low back pain specifically. Other definitions of 'back pain' as a whole include the area between the gluteal folds and vertebra prominens ${ }^{(7)}$ (at the base of the neck) ${ }^{(13)}$.

Despite this controversy in anatomical boundaries, the site boundaries of the area studied in this thesis are defined in section 2.4 , in conjunction with other aspects.

\subsubsection{Symptoms}

Having purported that low back pain is a symptom, it is important that any definition includes a description of what sensations are included within the anatomical boundaries. 
Therefore it is necessary to ascertain whether the study is just considering sensations of discomfort or pain, or whether spasm, tenderness, paraesthesia, anaesthesia, or 'cramp', are also valid inclusion criteria. If the symptoms to be included are not specified, it might be presumed that every sensation that is perceived by a participating client would be included in the study, which may not be intended. Once again, without this specificity of definition, comparisons between studies become meaningless.

For the purposes of this thesis, pain was the only aspect under review. Any other sensations mentioned by clients were recorded in the field notes for completeness, but were not specifically measured or analysed.

\subsubsection{Exclusion criteria}

Like the anatomical boundaries and symptoms, specific exclusion criteria are rarely defined in low back pain studies. Even when they are stated, they are often poorly defined and non-standardized, for example Biering-Sørensen excludes pain 'in relation to menstruation'(14), but does not specify whether this includes pre-menstrual low back pain, and Nagi et al. exclude low back pain arising from pregnancy ${ }^{(15)}$, but do not define whether this includes post-partum low back pain.

Another issue which is often unclear in studies of low back pain, surrounds the inclusion of thoracic pain: According to $\mathrm{Horal}^{(16)}$ and Nohejl et al. ${ }^{(17)}$ the lifetime incidence of thoracic spinal pain is around $20 \%$, meanwhile Hult estimates that about $4-5 \%$ of the population reports thoracic pain without low back pain ${ }^{(18)}$. Therefore, in epidemiological studies, if it remains unclear whether thoracic pain and low back pain are included in incidence and prevalence estimates, this can result in confusion and inaccuracies, (particularly if the anatomical boundaries are not stated).

\subsection{What is acute low back pain?}

Having considered what constitutes low back pain, a further classification is frequently used in the literature, based on the duration of symptoms. Various terms are used, for example, Cassidy and Wedge describe transient, acute, recurrent and chronic categories in their 'taxonomy of low back pain'(19). Some researchers include a 'subacute' category, 
while others such as Burton ${ }^{(20)}$ prefer the term 'subchronic' to subacute. Neither the terms used, nor their definitions are consistent.

The Quebec Task Force on Spinal Disorders adopted the definition of acute pain as being 'restricted to 7 days duration'; subacute 'from 7 days to 7 weeks'; and chronic pain as 'lasting more than 7 weeks' ${ }^{(12)}$. However, once again, these definitions are not universal, and such labels may not reflect the dynamic variation that exists within each category. As an illustration, acute low back pain of one-day duration may be vastly different to acute low back pain of six days duration, even in the same individual. As an alternative to defining acute back pain as lasting seven days, Magora refers to pain lasting three days or more ${ }^{(10)}$, whilst others specify the duration as less than three months $s^{(21,22,23)}$ - a considerable variation in time scales, especially considering the natural history of resolution of this symptom, which is discussed in section 2.7 .

Despite inconsistencies in terminology, in order for meaningful comparisons to be made in research, it must be clear what is being studied, and therefore any definition of low back pain must include a time-scale. It may be preferable to state the duration, rather than rely on arbitrary labels such as 'acute' and 'chronic', which are often wrongly assumed to be consistent within the literature. This suggestion may not be universally popular, as some authors argue that it is important to differentiate between acute, recurrent and chronic presentations, because evidence suggests that treating chronic back pain as if it were an episode of acute back pain can result in perpetuation of disability ${ }^{(24)}$.

An alternative to specifying the reported duration of symptoms, is expressing any sickness absence e.g. The Quebec Task Force for Spinal Disorders define 'the proportion of workers who were compensated with absence from work of at least one day for a spinal disorder' ${ }^{\prime(12)}$. This has the advantage of indicating the duration that a client's function was impaired (although it is not a particularly accurate outcome measure), but it fails to record this duration for clients such as retired clients and home-makers, who are not working, and therefore not entitled to sickness remuneration.

\subsection{Classification of back pain}

If the definition of 'acute low back pain' appears unclear, the aetiology and mechanisms of injury resulting in this symptom are even less well understood. Complex spinal 
anatomy, physiology and biomechanics challenge researchers and the non-specificity of many examination and diagnostic procedures further tests our understanding of low back pain $^{(1)}$.

Within the literature, opinion is divided as to whether it is possible to 'diagnose' and hence classify low back pain, based on this clinical diagnosis. This has resulted in a 'multiplicity of subclassification schemes'(25), where at one end of the spectrum, some believe that it is usually not possible (on clinical grounds) to assign a firm diagnosis to the symptom of low back pain ${ }^{(26,27,28)}$. Many of these authors consider that all such cases should be categorized simply as 'non-specific low back pain'. Here, the pain could arise from an acute muscle strain, damage to ligaments, intervertebral or pelvic joints etc. ${ }^{(29)}$. Fry argues that it is beyond the abilities of most 'normal clinicians' to be able to differentiate between these possible lesions ${ }^{(29)}$, whereas Klaber Moffett et al. attempt to quantify it, estimating that 'probably only about $15 \%$ of all cases of reported back pain can be definitely and reliably diagnosed ${ }^{\prime(13)}$. At the other end of the spectrum, Frymoyer et al. more optimistically contest that a proportion of cases, perhaps up to $50 \%$, can be reliably diagnosed ${ }^{(30)}$. However, this appears to be a minority view.

In keeping with this minority view that it is possible to classify back pain, some attempts have been made to produce elaborate subclassifications of non-specific low back pain $^{(31,32,33)}$. (For a comprehensive review of classification systems for back pain see Riddle $^{(34)}$.) However, such systems, according to Walsh, have not demonstrated that they reflect pathological processes, nor can they be reproducibly diagnosed by other observers $^{(1)}$. Walsh qualifies this opinion by saying the unsatisfactory attempts at classification are epitomized by the gold standard of classification, the I.C.D. (International Classification of Diseases), which describes in excess of 30 conditions which may result in back pain, with about 30 more that might include back pain, which suggests that low back pain classification is complex, poorly standardized and remains highly contentious within the literature ${ }^{(1)}$.

Despite this controversy, trends can be seen in some aspects of classification. For example, it is reported that over $90 \%$ of all clinically significant lower limb radiculopathy (due to disc herniation) involves the L5 or S1 nerve root at the L4/5 or L5/S1 disc level ${ }^{(22)}$, although precisely what other structures are involved cannot readily 
be determined clinically. Thus attempts at classification based on underlying pathology may be, at best, only partially accurate.

Interesting developments in the classification debate come from the Clinical Standards Advisory Group (C.S.A.G.) in the United Kingdom ${ }^{(35)}$ and the Agency for Health Care Policy and Research (A.H.C.P.R.) in the United States of America (U.S.A.) ${ }^{(36)}$, who have both attempted to classify low back pain using a triage system. Using the C.S.A.G. triage of: possible serious spinal pathology; nerve root pain; and simple backache ${ }^{(35)}$, the vast majority of clients form part of the 'simple backache' group, although this term has been criticized as this symptom is anything but 'simple'. Researchers have called for subgroups of this triage category ${ }^{(37)}$, arguing that due to the heterogeneity within this group, research findings are often inconclusive or contradictory ${ }^{(37)}$. To date this quest for subgroups has not resulted in universally accepted categories.

In addition to the obvious difficulties of classifying the physical component of low back pain, Deyo and Tsui-Wu state that cultural, social and economic factors may also influence the perception of pain and the response to $\mathrm{it}^{(3)}$. Valkenburg and Haanen go as far as describing the back as the organ system through which humans express their psychological burdens, when subconsciously they feel it necessary to do so ${ }^{(11)}$. This further adds to the challenge of studying low back pain, and perhaps it is little wonder that attempts at classifying back pain have to date been relatively unsuccessful.

Indeed there is a wider philosophical debate about whether such classifications should be undertaken at all, for example Nachemson advises against 'labelling patients' as he claims that this 'makes them ill'(38). He further warns clinicians that 'we are accelerating our ability to diagnose and treat clinically totally irrelevant pathology ${ }^{(38)}$. Surely this argument should be resolved before the details of any classification system are discussed?

\subsection{Operational definition}

Despite the complexities of defining and classifying back pain, it was necessary to have a working definition for the purpose of this thesis, so that it was clear exactly what was being studied. 
Therefore, considering the points discussed in sections $2.1-2.3$, acute low back pain was defined as an area bounded by the $12^{\text {th }}$ thoracic vertebra and $12^{\text {th }}$ ribs superiorly, gluteal folds inferiorly and contours of the trunk laterally. The duration of this symptom (pain), was defined by a client taking at least three days at home (off work) or equivalent, if they were not employed.

No attempt was made to classify any underlying pathology. The duration of 'at least three days' off work or equivalent was determined by the author and research team. This figure, although arbitrary, was chosen to ensure that the most minor episodes of back pain were excluded.

\subsection{The size of the problem}

Back pain is one of the most common and costly problems confronting health care providers and insurers ${ }^{(39)}$. To try and ascertain the size of the problem, some clues can be found in the epidemiological literature.

Epidemiology is the science concerned with the study of the frequency and distribution of disease in different populations and the various factors that influence these variables $^{(19)}$. In this literature, a number of different epidemiological measures are reported $^{(5)}$. Some studies investigate whether clients currently have back pain (point prevalence), some ask whether the client has experienced back pain over a period of time e.g. one year (period prevalence), whilst others seek to determine whether a client has ever had back pain (lifetime prevalence) - although some, incorrectly term this 'lifetime incidence $^{\text {(18,40)}}$.

Prevalence data do have their limitations. For example, cases with longer durations tend to be over-represented ${ }^{(5)}$, whilst cross-sectional surveys (point prevalence) can be misleading as they only give a snapshot of what is actually happening within a population. Meanwhile period prevalence and lifetime prevalence both rely on clients' memories, and all three types of prevalence can be affected by the exact wording of the questions asked. If, for example, prevalence data are collected at the end of August based on back pain in the previous three months, then those who have back pain aggravated by cold weather may give negative responses ${ }^{(7)}$. If, on the other hand, a retrospective enquiry covers a period of a calendar year, some episodes may well be 
forgotten ${ }^{(7)}$. In either case, such prevalence data often result in underestimation of the true occurrence of back pain.

Although prevalence data can be useful if interpreted with caution, a more robust epidemiological measure exists - incidence rates. These record the frequency of new episodes in a population per unit time ${ }^{(5)}$. Unfortunately they are not as commonly reported as prevalence data, presumably because they are best recorded by prospective studies which are usually more expensive and time-consuming to undertake than crosssectional surveys.

From the epidemiological literature, several studies have explored the occurrence of back pain. Table 2.1 summarizes the extremes of these incidence and prevalence rates reported in the literature for lifetime, point, two-week and one-month durations. In addition to the range of epidemiological examples shown in the table, one-year prevalence figures have been cited (in Denmark), as 43-48\% for males (aged 30,40,50 and 60 years) and $39-54 \%$ in similarly aged females ${ }^{(14)}$. Frymoyer et al. documented prevalence data over a three-year period by sampling 3929 medical records and identified that $11 \%$ of males and $9.5 \%$ of females reported low back pain during this period ${ }^{(47)}$. However, under-representation is common using this method, firstly, because it only samples people who have consulted a medical practitioner (unlike in the general population surveys) and secondly, because multiple complaints are often not documented individually at the consultation, hence the under-representation.

Findings from the studies shown in table 2.1 vary considerably as a result of differing definitions of low back pain, different ages and sexes of populations studied, variations in locations, methods of data collection, and the inclusion and exclusion criteria used. 


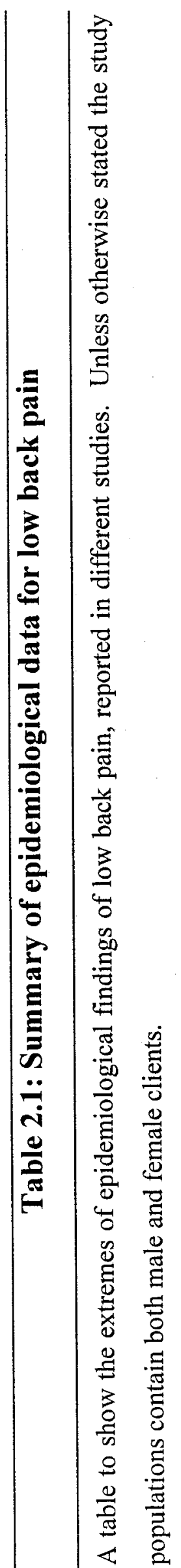

\begin{tabular}{|c|c|c|c|c|c|}
\hline \multirow{4}{*}{ 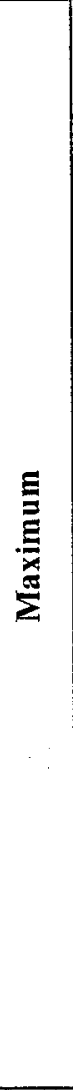 } & 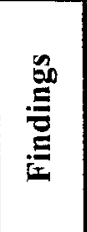 & 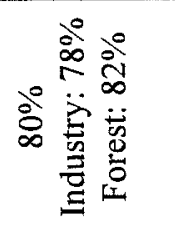 & 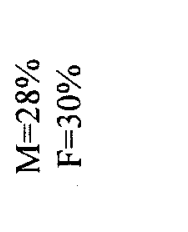 & $\stackrel{\partial}{\stackrel{0}{v}}$ & $\stackrel{\circ}{\stackrel{\circ}{m}}$ \\
\hline & 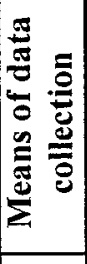 & 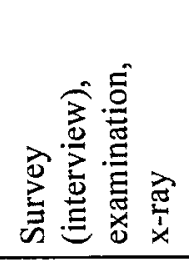 & 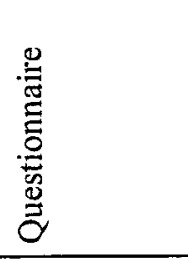 & . & 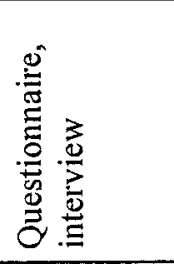 \\
\hline & 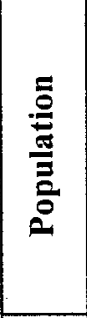 & 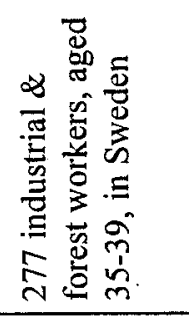 & 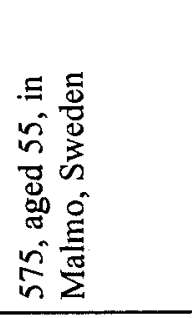 & 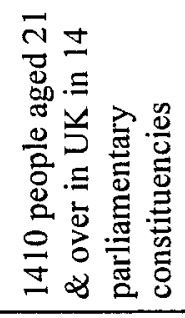 & 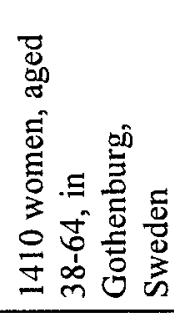 \\
\hline & 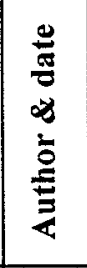 & 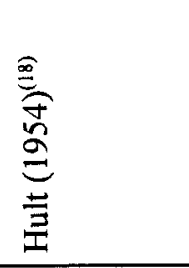 & 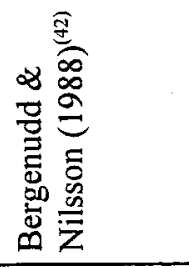 & 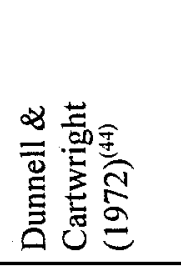 & 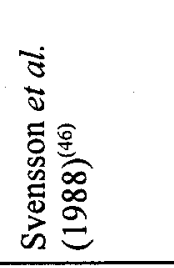 \\
\hline \multirow{4}{*}{$\stackrel{\Xi}{\stackrel{E}{E}}$} & 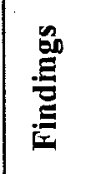 & 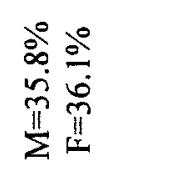 & 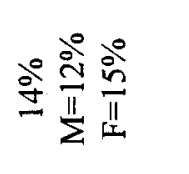 & 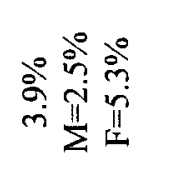 & 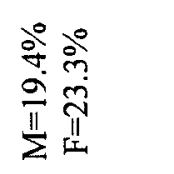 \\
\hline & 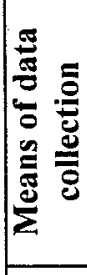 & 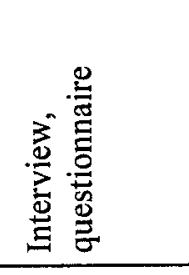 & 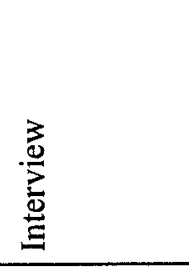 & 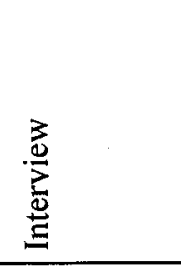 & 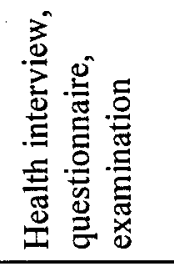 \\
\hline & 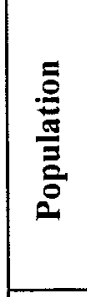 & 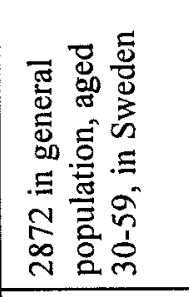 & 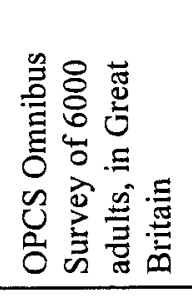 & 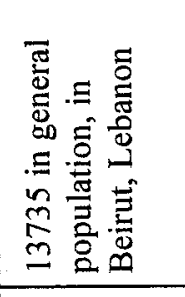 & 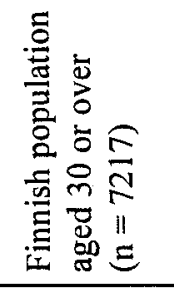 \\
\hline & 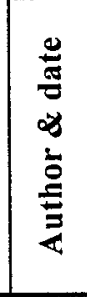 & 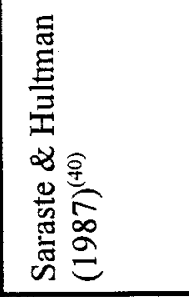 & 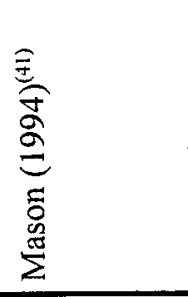 & 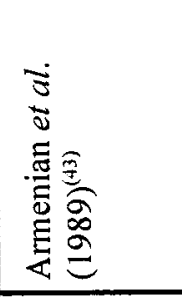 & 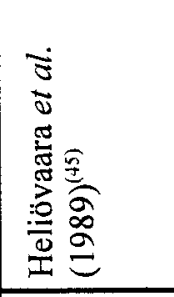 \\
\hline & & 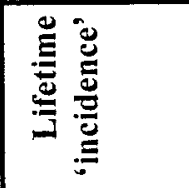 & 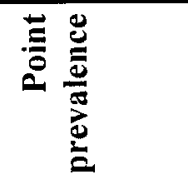 & 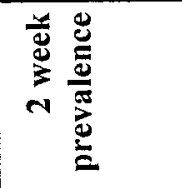 & 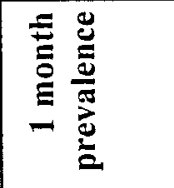 \\
\hline
\end{tabular}


The C.S.A.G. report has summarized epidemiological findings, stating that overall rates for point prevalence for back pain $=14-30 \%$, period prevalence (one-month) $=30-40 \%$ and in a lifetime $=60 \%{ }^{(48)}$. In practical terms, this makes back pain the second leading symptom that prompts visits to medical practitioners, (the leading symptom being respiratory) ${ }^{(3,23)}$. In G.P. surveys of morbidity in England and Wales, there is marked variation in consultation for back symptoms, which is attributed to differences in client behaviour once the symptoms have developed ${ }^{(49)}$. This is discussed further in section 3.1. Although the occurrence of low back pain is widely published and is reported not to have changed over time ${ }^{(50)}$, what is particularly concerning is the trend in increasing disability associated with back pain. During the past twenty years, the prevalence of disabling back pain for which benefits are paid has risen exponentially ${ }^{(51)}$. Evidence for this can be seen by comparing Sickness and Invalidity Benefit for back incapacity in Great Britain between 1978/9 and 1991/2, which indicates an increase of $208.5 \%$ over this period. By comparison, for all other musculoskeletal incapacities during the same period, the increase is $136.4 \%$, whilst respiratory diseases have decreased by $24.8 \%{ }^{(52)}$. The percentage rise in benefits claimed due to back pain is considerably greater than for other medical conditions and symptoms. This is not unique to the U.K. - it is a problem throughout the Western world. Perhaps the most striking statistic comes from Sweden where the permanent disability rate has increased by $6000 \%$ between the 1950 s and $1980 \mathrm{~s}^{(38)}$, a truly remarkable increase.

Much of this increase in disability associated with back pain is "probably due to an altered reaction to the problem, with increases in sick certification and state benefit perhaps reflecting patients' and doctors' expectations, concerns by employers, and social and medico-legal pressures ${ }^{\prime(53)}$. Characteristically employers will not take workers back until they are 'completely well' which usually means free of any pain ${ }^{(54)}$. This highly cautious approach not only has implications for employers and primary care management of back pain, but also impacts across all back pain services. For example, Nachemson says 'Even after surgery for disc hernia in the 50 s we all had $95 \%$ [of patients] returned to work. In 1990 we were down to $75 \%$ for the same operation. And the reason is that something has happened to society ${ }^{\text {(38) }}($ sic $)$. Of course, it could be influenced by other factors such as surgical technique, materials used and post-surgical regimes, which Nachemson does not consider. Nevertheless, whatever the reasons for these results, the 
implications for treating and managing this symptom are far-reaching, as are the cost implications.

\subsection{The cost of the problem}

According to Cassidy and Wedge, low back pain is usually the result of a benign process and is self-limiting in the majority of cases, but the sheer numbers of those affected are of major concern ${ }^{(19)}$. In addition, low back pain frequently affects people during their 'productive years'(2). The cost implications of this epidemic will now be considered globally and specifically.

The costs incurred for treating back pain can accumulate from many different sources. Obviously there are costs incurred from visits to general practitioners, plus any prescribed medication (e.g. analgesics, non-steroidal anti-inflammatory drugs, muscle relaxants, sleeping tablets, anti-depressants etc.). Additional costs may accrue from $\mathrm{x}$-rays, scans, blood tests, and referrals to out-patient physiotherapists, chiropractors, osteopaths, or consultants (who may be in orthopaedic, rheumatology, or neurosurgery departments, or pain clinics). Other costs may arise from accident and emergency attendances, in-patient episodes of care, and possible surgery, (which will incur after-care and rehabilitation costs).

As well as these costs to the National Health Service and private sector, there may be considerable financial burden on the individual, with costs arising from: pharmacological products (both prescription costs and over-the-counter medications); private treatments (e.g. manual and complementary therapies); alterations in lifestyle, (e.g. convenience foods, possible private domestic help, modified modes of transport such as taxis), all of which involve additional expense. Furthermore, some clients may seek other means of analgesia including alcohol, tobacco, or illegal substances, at considerable personal expense.

The cost of back pain does not stop with the expenditure for treatment already identified. Further financial burden arises from sickness benefits, loss of earnings, replacing wages and lost productivity, (both from in-patient and out-patient appointments). There are also costs incurred by occupational health teams, and costs to local government (including home helps, social work and meals on wheels $)^{(13)}$. Referrals for second opinions and 
medico-legal costs further increase this sum, and so it is not difficult to see why back pain has been reported to be such a major financial burden on society. Incidentally, this is only considering the costs of back pain once it has occurred, it takes no account of any costs incurred in trying to prevent back pain happening in the first place.

There have been two attempts in the literature to quantify the burden of back pain in the U.K. ${ }^{(13,35)}$, both of which need updating, and should be interpreted with caution, as they have some inaccuracies and omissions. The main findings are summarized in table 2.2.

\section{Table 2.2 Summary of the annual perceived costs of back pain}

Comparison of the two literature sources, which have costed the financial burden of back pain to society. The figures shown are expressed in $£$ million, unless otherwise stated.

\begin{tabular}{|l|c|c|}
\hline & $\begin{array}{c}\text { Centre for Health } \\
\text { Economics (1995) }\end{array}$ & $\begin{array}{c}\text { Clinical Standards } \\
\text { Advisory Group (1994) })^{(35)}\end{array}$ \\
\hline G.P. consultations & $67.3-99.4$ & 130 \\
\hline Prescribed drugs & $12.3-33.5$ & 48 \\
\hline Out-patient clinic visits & $12.3-24.3$ & 72 \\
\hline Physical therapy* & $24-36$ & 63 \\
\hline In-patient bed days & $117.4-122.4$ & 106 \\
\hline A \& E attendances & - & 17 \\
\hline X-rays & $26.7-60.1$ & 35 \\
\hline Private medical consultations & - & 144 \\
\hline Private physical therapy & 127 & 18 \\
\hline Over the counter medicines & $18-36$ & 3.8 billion \\
\hline Working days lost & - & 1.4 billion \\
\hline D.S.S. benefits & - & - \\
\hline Day cases (e.g. epidurals) & $5.3-7$ & - \\
\hline
\end{tabular}

* denotes costs for physiotherapy, chiropractic and osteopathy.

From the table, it is clear that differences exist between the two estimates, presumably arising from differences in the sources of literature used, data collection, analyses and interpretation of the findings. For example, estimates of the cost of medications are lower in the Centre for Health Economics (C.H.E.) figure than Clinical Standards 
Advisory Group (C.S.A.G.), since the former only includes four drugs: ibuprofen; cocodamol/coproxamol; Naproxen; and Voltarol. Clearly may other drugs are prescribed for back pain episodes, including anti-depressants, muscle relaxants etc., which are considered in the C.S.A.G. report, hence the higher estimate.

The C.H.E. data are derived from a literature review of Medline, Social Science Citations, PsychLit and AMED databases ${ }^{(13)}$, although the key words and years covered were not stated. This information was embellished with 'surveys for the estimation of the cost of G.P. consultations and physiotherapy', information obtained from clinicians, providers and purchasers ${ }^{(13)}$. Information was gathered on the 'direct costs' of back pain, and then 'adjusted to $1992 / 3$ prices', presumably to ensure that estimates were consistent and recent at the time the report was written. It is worth noting that the report does not address the 'indirect costs' of back pain such as production losses. ${ }^{(13)}$, which can be considerable. As an indication, in one study, medical costs accounted for only $33 \%$ of the total workers compensation costs, with the rest going towards disability payments ${ }^{(19)}$. Of the $67 \%$ disability payments, $22 \%$ (of the total) were temporary disability costs, whilst $45 \%$ were permanent ${ }^{(19)}$. However, it must be said that this was an American study, and as yet, the disability and compensation costs in the U.K. have not reached the levels reported in the American literature.

In contrast, the C.S.A.G. report was based on four main sources of information: 'a review of epidemiological evidence'; an 'up-to-date survey of 6000 adults in Britain'; a 'comprehensive study of GP and health care use by 8000 adults in the Manchester area'(sic); and 'DSS statistics on Sickness and Invalidity Benefit over a 40 year period'(sic) ${ }^{(35)}$. The C.S.A.G. authors have criticized the C.H.E. review for being 'based on a limited review of the epidemiological evidence'(35).

What is emerging from these two reviews is that little current, comprehensive evidence has been reported from U.K. populations, and thus comparisons with populations in other countries are limited due to differences in health care systems and recompense for incapacity resulting from back pain. Consequently, the authors of the C.H.E. report consider it is not possible to place an exact figure on the cost to society ${ }^{(13)}$. Regrettably, to date no study has attempted to estimate the cost of back pain to the individual. 
In trying to ascertain information about the cost of back pain in smaller, (more manageable) populations, figures have been calculated for work lost due to low back pain in individual organisations. For example, in an Eastman Kodak Plant in Rochester, New York, it was found that over a ten year period, $45 \%$ of 'heavy handlers' and $35 \%$ of 'sedentary workers' visited the medical department because of low back pain. The time lost to the organisation amounted to four hours for every employee per year. This figure was second only to the hours lost from upper respiratory tract infections ${ }^{(55)}$.

The Industrial Society (in the C.H.E. report) includes information about how absence due to back pain is perceived by managers. Managers perceive back problems to be the third most commonly reported reason (given by employees) for absence, after colds/influenza and stomach upsets/food poisoning ${ }^{(13)}$. What is particularly interesting is when the same managers were asked what they thought the real reasons were for absence, back pain moved down to sixth place on the list ${ }^{(13)}$. This suggests that managers are sceptical, believing that malingering is a common practice, although this is not born out in the literature ${ }^{(13)}$. It is also not clear where this 'malingering' image has originated from, nor how it has become such an apparently common perception amongst managers.

One particularly striking finding from the literature is that the cost of chronic back pain (usually defined as being in excess of six months duration), is responsible for the majority of the total costs attributed to low back pain. In a study of back injury claims at the Boeing Company in Washington, $10 \%$ of the 900 back injury claims over a fifteen month period accounted for $79 \%$ of the total back injury costs ${ }^{(19)}$. This is consistent with the findings from the Quebec Task Force for Spinal Disorders who revealed that the $7.4 \%$ of employees who were absent from work for six months or more accounted for $75.6 \%$ of the total compensation costs for spinal disorders at the Quebec Workers Compensation Board ${ }^{(12)}$.

The amount of economic loss resulting from low back pain justifies the expenditure of research money to tackle this problem ${ }^{(1)}$. It is essential that factors are identified which predispose 'acute' and 'subacute' low back pain to becoming 'chronic', thus minimizing the enormous drain on finite resources that chronic back pain causes. 


\subsection{The natural history of low back pain}

Having examined how frequently back pain occurs and the financial implications, it is important to consider the natural history of this symptom, partly to help understand the likely prognosis (if no interventions were made), and then to help clinicians focus on the particular point when their intervention is likely to be most effective. A number of pertinent questions arise, including: whether back pain is self-limiting?; how soon does the pain settle?; and how soon are clients able to return to full function?

Among the general population, it is reported that little is known about the precise natural history of low back pain ${ }^{(11)}$. Certainly many people will know others who have experienced this symptom, and may therefore be influenced by these experiences.

From the medical literature, many studies report the natural history of back pain. In addition, generalizations about its natural history of resolution appear in most clinical guidelines $^{(12,22,35)}$. This started in 1987 with the Quebec Task Force for Spinal Disorders, who reported that $74 \%$ of compensable back pain resolves within four weeks and $87 \%$ within three months ${ }^{(12)}$. The authors conclude that in only $5 \%$ of cases, back pain persists for six months or more ${ }^{(12)}$. Until recently, such reports of this rapid natural history of resolution were relatively invariant across several countries ${ }^{(5)}$. Indeed, some authors cite even more optimistic findings, such as Cassidy and Wedge, who report that $90 \%$ of clients with acute low back pain improve after two months, and describe the percentage of people with symptoms declining to $2-3 \%$ after six months ${ }^{(19)}$. By one year, this figure further decreases to $1 \%{ }^{(19)}$.

However, such favourable findings give way to recent, more concerning evidence, such as the C.S.A.G. report (in 1994), which recognizes that the earlier estimates of the rate of recovery were 'over-optimistic and over-emphasised return to work' ${ }^{\text {(35) }}$ (sic). In these guidelines, the authors conclude that $50 \%$ of back pain episodes settle 'more or less completely' within four weeks, but $15-20 \%$ of clients continue to show some symptoms for at least one year ${ }^{(35)}$. Nevertheless, the focus of the report is still very positive: 'Backache has a good natural recovery rate. There is a $90 \%$ probability that an attack will settle, at least sufficient to return to work within six weeks ${ }^{(35)}$. Although the authors advocate a clear tendency towards natural recovery, they warn that this should not be grounds for complacency ${ }^{(35)}$. 
More recently, Croft et al. (in 1998), specifically challenged an earlier claim that $90 \%$ of episodes of low back pain in general practice have resolved within one month ${ }^{(51)}$. In a prospective study of 490 clients (in two general practices) who presented with low back pain in a 12 month period, only $21 \%$ of clients were 'completely recovered' in terms of pain and disability by three months, and at 12 month follow-up, this proportion had only marginally improved to $25 \%{ }^{(51)}$. The authors conclude that clients have stopped consulting with symptoms within three months, although 'most will still be experiencing low back pain and related disability one year after consultation'(51).

There is a considerable shift that has occurred from estimating that $74 \%$ of low back pain episodes resolve within four weeks, a decade ago, to $75 \%$ not being completely recovered by 12 months, now. There could be several reasons for this. Firstly, it could be that the natural history of back pain is changing and people are now more likely to experience symptoms for longer. Anecdotally, this could be due to psychosocial factors such as stress, or lifestyle factors such as taking less physical exercise or changing working practices. It may be that as a result of changing lifestyle factors, when injury occurs in the spine, the associated tissue changes are greater, and hence take longer to restore full function. For example, in clinical practice, clients with neck pain often report that periods of prolonged computer use, and stressful work schedules, results in localized tenderness in the upper fibres of the trapezius muscle. Stress can be a factor that activates localized trigger points in the muscle, which either initially causes, or increases existing pain. It is possible that similar mechanisms exist in the lumbar spine, which result in low back pain.

Secondly, these differences in reported natural history could be due to differences in methods and sources of data. For example in the Quebec study, two sources of data were used from the Quebec Workers' Compensation Board - computerized files and clinical records ${ }^{(12)}$. The level of agreement between these two sources was only $63.5 \%$, i.e. $36.5 \%$ of compensated claims related to the back and neck were misclassified in the computerized files, thus under-estimating epidemiological findings ${ }^{(12)}$. In addition, the outcome measure reported in this study was return to work, but this is not a measure of resolution of symptoms. By comparison, Croft et al. specifically defined resolution of pain as scoring 0 or 1 on a visual analogue scale, and resolution of disability as scoring $>90 \%$ on the Hanover back pain daily activity schedule, which asks about the ease of 
performing 12 everyday activities in the previous week ${ }^{(51)}$. Although it is not clear how these definitions were established, they are a more sensitive outcome measures than whether or not clients have returned to work, and thus are likely to classify fewer clients as asymptomatic, which is reflected in the figures.

Thirdly, there may be differences in clients' levels of acceptance of pain and disability over a period of time. As medicine and science advance, people are likely to have a greater expectation of a cure, and thus more readily voice their residual symptoms when this expected cure has not occurred. Anecdotally, from clinical experience, sadly advances in the management of low back pain have not kept pace with advances in clients' expectations and demands.

Nevertheless, from reviewing the natural history of low back pain, it is evident that back pain has a 'good natural recovery rate'(35), although this can still mean considerable discomfort and dysfunction for a person who is experiencing it. It is perhaps not surprising that only $25 \%$ of clients in Croft et al.'s study were 'completely recovered' by 12 months $^{(51)}$, when the healing time-scale proposed by Evans ${ }^{(56)}$, for soft tissue injuries is considered. After all, these injuries form the majority of back pain episodes, and Evans states that remodelling of new collagen, following inflammation and healing, continues for six months or $\mathrm{so}^{(56)}$. Therefore, how can earlier estimates of natural resolution (such as the Quebec Task Force who estimated that $74 \%$ of episodes resolve within four weeks ${ }^{(12)}$ ), be accurate, based on a physiological argument? As Evans says 'Healing cannot be accelerated. But if we know the basic mechanisms of repair, we can tell patients how to avoid delayed or poor recovery' $(\text { sic })^{(56)}$.

\subsection{Recurrences of low back pain episodes}

Although it has been reported that back pain has a good natural recovery rate ${ }^{(35)}$, it is important to ascertain its rate of recurrence. This is not straight-forward, as it can be difficult to define precisely when an episode starts and ends, or when further symptoms constitute a new recurrence rather than a continuation of symptoms ${ }^{(48)}$. For a summary of recurrence rates, see the C.S.A.G. Epidemiological Review (1994), which concludes that '70\% of people who ever experience an attack of back pain will suffer three or more recurrences but recurrences may tend to settle over several years ${ }^{\text {(48) }}$. 
The New Zealand guidelines advocate treating recurrent episodes in a similar way to acute low back pain episodes ${ }^{(22)}$, although this may not be appropriate as it cannot be assumed that the psychosocial factors in acute and recurrent pain are necessarily similar, such as clients' expectations of recovery, attitudes towards self-help and seeking medical care. These aspects must be considered alongside any physical symptoms.

\subsection{Returning to work}

Within the medical literature, there has been great emphasis on getting people back to work before they are completely asymptomatic, because it has been reported that once someone with backache is off work for six months, they have only a $50 \%$ chance of returning to their previous job ${ }^{(35)}$. Worse still, 'once they are off work for two years, or have lost their job because of back pain, ... they will have great difficulty ever returning to any form of work' ${ }^{(35)}$.

In the light of the recent findings about the natural history of back pain, encouraging clients to resume work before they are completely asymptomatic is justified, otherwise, with $75 \%$ still not 'completely recovered' after one year ${ }^{(51)}$, the majority of these may not return to their previous job, which has considerable financial implications for the individual, their employer and society as a whole.

In addition, with support from their employers, many people with resolving back pain can resume at least some aspects of their job. Managers must not discourage workers from resuming activities with some residual symptoms, for fear of litigation. Greater understanding of back pain and closer links with health professions (preferably through occupational health departments), would facilitate this process, and enable clients to return to work.

\subsection{Conclusions from the back pain literature}

So far, this chapter has considered the importance of defining acute low back pain, so that findings from research studies can be meaningfully utilized.

From sections 2.5 and 2.6, it is evident that low back pain is common, costly and the disability associated with it is rising exponentially. Indeed, Waddell describes back pain 
as a ' $20^{\text {th }}$ century medical disaster' ${ }^{\text {'(57) }}$. Hence research which investigates this symptom and aims to reduce the misery and disability associated with it, is much needed.

From the reports on the natural history of resolution, it is clear that many episodes of low back pain improve considerably within the first four weeks, but we then need to address why some clients' back pain apparently resolves, whilst in others, this does not appear to happen. Perhaps some of the answers may lie in the psychological make-up of the individual? Some of these issues will now be explored in the remainder of this chapter.

\subsection{Control issues}

There are many different perceptions of health and illness, and many proposed underpinning concepts. For decades, social scientists have constructed models and theories to help explain different notions of control, such as locus of control, perceived control, self-efficacy and health beliefs.

One term that often appears in the psychosocial literature is personal control, which has been studied for more than half a century ${ }^{(58)}$. It is claimed to be one of the most important ways in which people differ from each other ${ }^{(58)}$, and is 'the belief that it is possible to respond in some way, to influence the aversiveness of an event ${ }^{\text {(59) }}$. It is therefore both a cause and a consequence of the way people respond to their environment ${ }^{(58)}$.

The concept is not set in stone, but can be influenced by factors such as success and failure $^{(58)}$. It is a concept whose links with health are well established ${ }^{(58)}$, since it is important to understand whether clients experiencing acute low back pain feel they have any personal control over their symptoms i.e. 'Will it hurt less if I can control it?'(Thompson in 59). If clients perceive themselves to have little control over their symptoms, how does their rate of recovery compare to those clients who perceive themselves as having more personal control? Such information could be useful in giving clients advice, treatment and estimating the likely prognosis of their symptoms. 


\subsection{Social learning theory}

Before looking at some of the constructs of personal control, it is worth considering the origins of this work. One important theory, which has had a major impact in this field, is the social learning theory $-\mathrm{a}$ 'personality' theory, originally developed to try and explain human behaviour in complex social situations ${ }^{(60)}$.

Some (incorrectly) attribute this theory to Bandura ${ }^{(62)}$ who is reported to have renamed the theory 'social cognitive theory' ${ }^{(63)}$, but it was in fact Rotter who outlined much of the original concept many years earlier, in the early 1950s.

Although it is considered a theory of personality, Rotter et al. recognize that there is no entity called 'personality', but that there are simply many different constructions about the nature of a person, that lead to different definitions of personality ${ }^{(60)}$.

Social learning theory was an important development as it attempted to integrate reinforcement and cognitive theories ${ }^{(60)}$. It provided a tentative set of principles to account for complex human behaviour that differed from these earlier conceptual systems. In recognizing this development, Rotter et al. state 'In many ways, conceptual systems are like tinted glass. Each provides a view of the world which is slightly different from the other. Change your glasses and the world changes. Everybody has a look through the glasses of some conceptual system in order to view reality. It is difficult but important to remember that one is not viewing reality directly but only through the intervening conceptual system ${ }^{,(60)}$.

Social learning theory uses an historic approach (since its authors maintain that to understand a personality, it is necessary to investigate antecedent events in a person's life $\left.{ }^{(60)}\right)$. Using this approach, the theory implies that individuals have a choice in how they will behave, and before deciding on a particular action, they must consider 'both their valuation of the outcome (reinforcement value) and their estimation of the likelihood or probability of its occurring (expectancy) ${ }^{,(61)}$.

In Rotter's theory, essentially there are four basic components that are utilized in the prediction of behaviour: behaviour potential; expectancy; reinforcement value (where a reinforcement is defined as anything that reduces a need $\left.{ }^{(60)}\right)$; and the psychological 
situation, which all contribute to predicting the cognitive processes that are related to behaviour $^{(60)}$.

Behaviour potential is a relative concept, which considers the likelihood of a person's behaviour occurring in a given situation with a certain set of circumstances. The behaviours may be directly observed or implicit ${ }^{(60)}$. For example, the behaviour potential of a person experiencing an acute episode of low back pain might consider the likelihood that the person uses a lumbar support in the small of their back when sitting. This concept would also include implicit behaviours such as rationalizing the need for this behaviour and considering other alternatives.

Expectancy is defined as the probability held by the individual that a particular reinforcement will occur as a function of a specific behaviour on their part in a specific situation $^{(60)}$. In the case of the person experiencing back pain, the expectancy could be the probability that the back pain will lessen when a lumbar support is used in sitting.

Rotter defines the reinforcement value as 'the degree of the person's preference for that reinforcement to occur if the possibilities of occurrence of all alternatives were equal ${ }^{(60)}$. This relative term refers to a preference, and in the case of the person with back pain, the reinforcement value of them taking their jumper off and rolling it up in the small of their back for support, may be compared with the activity of standing up, if both behaviours were equally possible.

The 'psychological situation' recognizes that behaviour does not occur in a vacuum, and that a person continuously reacts to aspects of their external and internal environment ${ }^{(60)}$. As Rotter et al. acknowledge: "While it may be true that person A is generally more aggressive than person $B$, nonetheless, there can arise many occasions on which person $B$ behaves more aggressively than does person $\mathrm{A}^{(60)}$.

Rotter defines these four basic concepts and attempts to link them in order to predict behaviour and the internal or cognitive processes related to behaviour ${ }^{(60)}$. The key to the theory is trying to identify when one form of behaviour is chosen over another in a specific, complex situation. Rotter et al. acknowledge that the goal of social learning theory is not to provide the 'facts' of personality, but, rather to provide a vehicle for the conceptualization of the facts ${ }^{(60)}$. 
It has been argued that the strength of this theory lies in its value as a method of analysis of psychological problems in personality and related areas ${ }^{(60)}$. However, as with any psychological theory, there are shortcomings. For example, Leventhal et al. criticize the social learning theory for attempting 'to explain the process underlying choices in the absence of knowledge of the constituents of the choice process ${ }^{\text {(64) }}$. In this criticism though, Leventhal does not make it clear whether he thinks this was an oversight, an assumption or an over-simplification made by Rotter during this early work. Another criticism of this theory is that its complexity may hinder analysis and interpretation. It also fails to account for the origin of private opinions that are not socially acceptable ${ }^{(101)}$, and it does not discriminate between desirable and undesirable learning ${ }^{(101)}$. It does however, focus on the social aspects of learning, acknowledging the complexity of the interaction between a person and their environment ${ }^{(101)}$, which is a useful foundation on which to build psychological constructs.

\subsection{Locus of control theory}

From the social learning theory and personal control work, many psychological constructs have evolved, such as locus of control and self-efficacy. These constructs have been developed to try and explain perceptions of health, and many theorists and researchers support the notion that 'the state of one's psyche influences one's physical health and the state of one's physical health influences one's psyche ${ }^{9(65)}$. This also affects how beliefs relate to behaviour - a vital concept when considering control issues.

Therefore the physical and psychosocial aspects of a person are inter-linked and thus cannot be studied as two separate entities. This is particularly important with low back pain, since many emotions are associated with this symptom, most commonly: fear; anxiety; increased body awareness; anger; and depression ${ }^{(66)}$. It may be that the emotional stages which clients with low back pain experience, are similar to the five stages proposed by Kübler-Ross i.e. 1) denial 2) anger 3) bargaining 4) depression and 5) acceptance ${ }^{(67)}$. Originally this work was written around the topic of dying and although low back pain is not life-threatening (except in very rare circumstances), from clinical experience, these emotional stages are commonly seen in clients, especially if their symptoms are long-standing. 
Some clients use the primary defence mechanism of denial (particularly if there are degenerative or age-related changes) in their spine. Anger may be focused towards the client's body, or the person or circumstances which they consider may have caused or worsened their symptoms. Sometimes this anger may manifest itself against health professionals, or medicine in general, if clients perceive that nothing can be done. According to Ruark, bargaining consists of 'an effort to retain the illusion of control in a situation in which one is powerless ${ }^{\text {(67) }}$, and this stage may involve negotiations about treatment options. In Kübler-Ross' fourth stage, depression, clients may experience mood swings, which may arise from a sense of loss of function and self-reflection on what the client used to be able to do. They may progress to the final stages of accepting their symptoms, however this is not a one-way passage through the five stages, the client may still show signs of the other stages at any time.

There is a cycle of interactions between such emotions and the physical signs and symptoms which affects clients' behaviour and as Leventhal and Crouch state, 'the behaviours that we adopt to maintain health and avoid and control disease reflect our medical and psychosocial histories ${ }^{(68)}$. Therefore these behaviours are both complex to understand and challenging to measure, and perhaps reflect the reasons why a 'cure' for acute low back pain has not been found.

Locus of control, a theoretical construct which evolved from the social learning theory, has attracted much attention as it 'has shown promise as a determinant of behaviour ${ }^{\text {(69) }}$. The construct depicts the degree to which a person believes that he possesses or lacks the power to control what happens in life circumstances ${ }^{(70)}$.

Individuals who believe that what happens to them is primarily due to their own actions or attributes are characterized as having an internal locus of control while those who believe that what happens to them is principally because of luck, fate, chance or other (powerful) individuals, or is unpredictable because of the complexity of the situation are considered to have an external locus of control ${ }^{(61)}$.

Although people with an internal locus of control do not necessarily differ from those with an external locus of control in either intelligence or learning achievements, many claims are made about the effects of internality. 
In two reviews, Arakelian ${ }^{(61)}$ and Strickland ${ }^{(71)}$ have summarized these claims, some of which relate to personality characteristics, some to health issues, whilst others relate to information processing. Examples of apparent personality attributes of people with an internal locus of control (when compared with more external tendencies) include: more motivation $^{(61)}$; greater trust in strangers ${ }^{(61)}$; more willing to approach, use and benefit from assistance from authority figures ${ }^{(61)}$; likely to exhibit denial when confronted with a threat ${ }^{(61)}$; usually better able to forego immediate rewards in favour of more valued, longterm goals ${ }^{(61)}$. In relation to health, people with an internal locus of control are apparently: more likely to engage in preventive activities such as dental care, contraception and inoculations against influenza ${ }^{(71)}$; more likely to have positive attitudes towards physical exercise and cardiovascular fitness ${ }^{(71)}$; better liked and more persuasive, and have thus been shown to influence health care professionals' decisions ${ }^{(61)}$. They are also more likely to: collect information about disease and health maintenance when alerted to possible hazards $e$. . hypertension $^{(71)}$; be successful in initial mastery of health information $^{(61)}$; and better able to extract relevant information or situational cues and use these data in effective problem solving ${ }^{(61)}$.

The belief in internal or external control is thought to be a relatively stable personality characteristic $^{(69)}$, which is often portrayed as a variable concept that exists on a continuum $^{(61)}$. Paulhus and Christie portray Rotter's construct as unidimensional, simply represented as:

Rotter model( ${ }^{(/ 2)}$

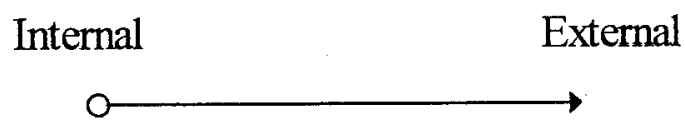

The belief in internal or external control as a relatively stable personality characteristic, comes from the notion that locus of control is a 'generalised expectancy' i.e. something that a person carries from one situation to the next $\mathrm{t}^{(73)}$. Therefore, Wallston has described it as "more trait-like than state-like and is therefore akin to a "personality" dimension' $^{\text {'(73) }}$. However this notion has been challenged by researchers, and some of the arguments are now considered. 
People are not fixed at one point along the continuum as 'internals' or 'externals', it depends on a particular situation. It may be that some individuals feel particularly empowered and perceive themselves as being in control of some aspects of their life, for example finances, whereas they may not perceive themselves in control of other aspects, such as health.

In the social science literature, little emphasis has been placed on situational factors that may (temporarily) affect a person's expectancies regarding locus of control ${ }^{(74)}$. Smith hypothesized that in times of a crisis, clients would be overwhelmed by external factors in their lives, and would initially be more externally oriented than a similar group of clients not experiencing a crisis ${ }^{(74)}$. He proposed that the crisis group would then show a significant shift towards the internal end of the dimension during the period of crisis resolution, whilst the non-crisis group would show no such shift ${ }^{(74)}$. In a study of 30 crisis clients and 30 non-crisis clients (where the crisis group consisted of clients who presented at a neuropsychiatric emergency centre because of 'acute life crisis'), the results supported this hypothesis, suggesting that the locus of control construct is transient, and can be affected by situations or circumstances ${ }^{(74)}$. However, the study used Rotter's I-E scale, and thus considered the locus of control construct as unidimensional, which has since has been superceded by the notion of a multidimensional construct (see below). It would be interesting if Smith's study was repeated using multidimensional locus of control outcome measures.

Like Smith's work with crisis clients, McCreary and Turner suggest that the locus of control construct is a transient attribution for clients in pain, affected by personal and social factors, as opposed to a consistent disposition across situations (trait) ${ }^{(75)}$. However, this view is not universal, since for example Aasen believes the locus of control construct to be relatively stable over time and place ${ }^{(76,77)}$.

In addition, even for the same situation, a person's locus of control can change over time by introducing new experiences that alter previous patterns of success and failure ${ }^{(61)}$. Indeed Rotter et al. state that generalized expectancies such as locus of control orientation are particularly predictive in novel situations, but as the person gains experience in specific situations, the predictive power of generalized expectancies decreases $^{(78)}$. 
Keefe and Brown concur with this view, arguing that the locus of control construct moves with time ${ }^{(\mathrm{in} 79)}$. They suggest that as clients with pain proceed from the acute to the prechronic (subacute), and finally the chronic phases of a pain problem, their perceptions of ability to control pain decrease $\left.{ }^{(i n} 79\right)$. It is interesting to consider whether this occurs with all chronic symptoms and indeed what happens in conditions with a rapid natural history of resolution, such as acute low back pain.

In addition to an individual's locus of control perceptions changing over time, it has been questioned whether the views of society in general have remained constant - indeed, Ponto questions the relevance of the normative values for Rotter's I-E Scale (which were established in 1966), to a student population in whom she was measuring locus of control and satisfaction ${ }^{(80)}$. To date, little work is reported in this area.

Despite Rotter's development of the locus of control construct from the social learning theory, his unidimensional model was criticized for being too simplistic, and so Levenson added a new dimension by differentiating between two types of external orientation. She identified a belief in the basic unordered and random nature of the world (chance), which was distinct from a belief in basic order and predictability of the world which was coupled with the expectancy that others are in control (powerful others) ${ }^{(81)}$. This can be shown diagrammatically:

\section{Levenson's model(1/)}

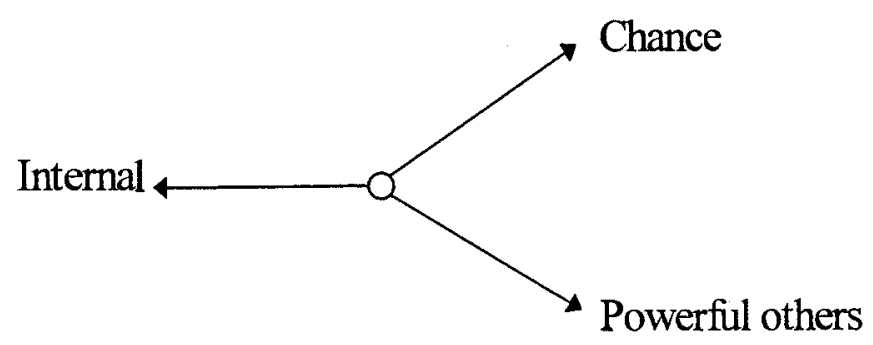

Now the locus of control construct was deemed multidimensional, and has been widely used in the field of health. The experience of personal control has vast implications for human behaviour ${ }^{(82)}$, and the general hypothesis behind health locus of control is that people who feel in control of their own health are likely to engage in healthy behaviour 
and follow the advice and recommendations of health authorities and agencies, whereas those who do not feel able to control their own health, will be less likely to do $\mathrm{so}^{(83)}$.

Specific behaviours where locus of control is relevant include seeking information, taking medication, making and keeping physician appointments, maintaining a diet, giving up smoking ${ }^{(84)}$ and seeking 'alternative therapy' $(\text { sic })^{(59)}$. Internals generally show more positive behaviours in each of these areas, but contradictory evidence exists (for a review see Wallston and Wallston) ${ }^{(84)}$.

Despite the development of the multidimensional construct, it cannot just be assumed that this automatically supercedes the unidimensional construct, indeed McCreary and Turner challenge whether it is better to use the multidimensional or unidimensional to measure the locus of control construct ${ }^{(75)}$. They conclude that 'Rather than searching for the "correct" measure, it may be important to use both procedures because in some populations and circumstances this dimension appears as a bipolar continuum, but not in other population / situation combinations ${ }^{3(75)}$. However they make no attempt to distinguish when the unidimensional approach might be more pertinent, and when the multidimensional.

\subsubsection{Self-efficacy}

Before considering specific applications of locus of control theory, distinctions must be made between outcome expectancies as measured by locus of control and behavioural expectancies or self-efficacy, a term first coined by Bandura ${ }^{(85)}$. This can be described as 'the sense that one's actions are effective and meaningful'(62). Individuals may feel responsible for their own health (internal locus of control), but at the same time perceive themselves to be incapable of performing the required activities (low self-efficacy) ${ }^{(86)}$. Therefore it is not enough to possess the relevant skills to enable a person to cope, the person must believe that they have them and are capable of applying them as needed ${ }^{(87)}$. This has been shown to have some practical benefit, since across a number of studies of experimentally-induced acute pain, higher beliefs in self-efficacy were associated with better coping ${ }^{(59)}$, but it must be remembered that there are important psychological and emotional differences between experimentally-induced pain and pain arising from a realworld, clinical source, and thus the interpretation of these two pain types are not directly comparable. 
It has been suggested that self-efficacy beliefs may go some way towards explaining the variability between a client's skill level and his or her performance outside the clinic ${ }^{(88)}$. In a study of 100 clients with chronic low back pain (of non-cancer origin), of 9.7 years mean duration, Strong examined which variables could predict a client's perceived functional status at the time of their admission to a pain management programme in Brisbane $^{(88)}$. The author concludes that the results 'have some important implications for the treatment of clients with chronic low back pain', claiming that the study found the contribution of self-efficacy, depression, reinterpreting coping strategies and gender were each significant in predicting the client's scores on the Pain Disability Index ${ }^{(88)}$. However, these findings should be interpreted with considerable caution, as there is evidence of 'data dredging' with the 12 outcome measures used which casts doubt on the analysis. This, coupled with the low correlation coefficients and small numbers of clients involved, renders the author's conclusions highly questionable.

As well as possibly shedding light on variation in clients' skill level and performance, self-efficacy may have a role in predicting outcomes of treatment - indeed Wallston is reported to advocate that beliefs about self-efficacy may be even more important than beliefs about locus of control in predicting these outcomes ${ }^{(i n 5)}$.

Despite these advantages, a number of shortcomings of the self-efficacy notion have been identified, including the notion that measuring self-efficacy depends upon a person's ability to consciously introspect ${ }^{(59)}$, and even if people are capable of making these judgements, they may only be able to do so in certain situations ${ }^{(59)}$. Furthermore, measurements of self-efficacy may be no more than statements of intention ${ }^{(59)}$, as they are not monitoring actual performance. Finally, there has been some debate about pain tolerance with this construct, since the predictions of self-efficacy theory contradict those of learned helplessness, and to address this, Bandura has argued that pain endurance is achieved through different mechanisms, "but this debate has not yet been resolved" ${ }^{(59)}$. Comparison and development of these various control constructs continues, and it is clear that there is no one construct that is universally adopted in the literature. 


\subsection{Variations in the locus of control construct}

Before looking at the locus of control construct in relation to back pain, it is important to look first at the trends that exist in relation to gender and age, as these may influence study findings. These two aspects will now be considered.

\subsubsection{Gender}

In a study comparing health control attitudes between clients with chronic low back pain in the U.S.A. with those in New Zealand, women in both countries rated themselves as having less personal control over health than men, using the Health Locus of Control Scale $^{(89)}$. Various reasons were proposed for this, including that 'aggression, activity, dominance, and self-esteem may be higher in males' while 'women may have been more vulnerable to a helplessness ... such as that presented by the experience of chronic pain ${ }^{\prime(89)}$. However such a bold statement assumes that both genders are equally honest in their self-assessment, and that they have comparable ability to evaluate their own personal control, which surely must be questionable.

In addition other factors may interact with perceptions of control, which again may be different between genders. For example Wallston and Wallston suggest that there may be an interaction between attributions to powerful others and level of interpersonal trust. They argue that individuals with a strong belief in powerful others and a tendency to distrust others might experience conflict when asked about psychological symptoms ${ }^{(i n}{ }^{90)}$. If this is the case, it cannot be assumed that males and females will necessarily experience the same degree of interpersonal trust, and so this could therefore influence perceived control. Clearly the debate about gender and perceived control is not as simplistic as Tait et al. ${ }^{(89)}$ suggest. This is considered in relation to the research in this thesis in section 7.2.1.

\subsubsection{Age}

According to Leventhal and Crouch, symptoms in the elderly are often slow to develop, less severe and longer lasting ${ }^{(68)}$. They appear against a more complex background of somatic sensations, and older people may have difficulty distinguishing illness specific symptoms from those attributed to normal ageing ${ }^{(68)}$. 
It is reported that age has been found to be related to control beliefs, but the findings have not been consistent ${ }^{(91)}$. In Smith's study involving crisis and non-crisis clients, previously mentioned, it was hypothesized that the magnitude of change from externality towards (relative) internality would be negatively related to age ${ }^{(74)}$. This was based on the assumption that stability of expectancies is a function of age $\mathrm{e}^{(74)}$, but the findings of this study, despite the small numbers of clients in each group $(n=30)$, did not support this hypothesis ${ }^{(74)}$. In addition, it must be remembered that the clients from the crisis group presented at a neuropsychiatric emergency centre, which may not be representative of the general population. Had the data been collected using a multidimensional measure rather than the unidimensional, it would have been interesting to compare the relative swing away from externality in the chance and powerful others subscales, in relation to age.

In contrast, Härkäpää et al. undertook a study where 459 clients with chronic and recurrent low back pain were surveyed and examined. Younger subjects (and males) were reported to be more internal in their beliefs ${ }^{(91)}$.

Likewise, in a study of 160 clients referred to a pain management centre with chronic pain of mean duration 5.3 years $^{(92)}$, younger male clients reported a stronger internal attributional style, whereas older male clients relied heavily on chance and powerful other factors ${ }^{(92)}$. The authors go further in saying clients who endorsed high scores on all three orientations in the Multidimensional Health Locus of Control Scale 'were likely to be older ${ }^{\text {(92) }}$. Unfortunately the authors offer no suggestions for why this might occur, and it does seem surprising. Perhaps it could occur as a result of using Likert scales where a minimum score equates with 'strongly disagree' and a maximum score equate with 'strongly agree'. This might suggest that older clients are generally more inclined to agree with statements and therefore be less likely to challenge them than younger clients? If this were the case, then this has implications for using the Multidimensional Health Locus of Control Scales across wide age ranges, as it may not truly be measuring locus of control orientation, but instead, an element of 'agreement' with a series of statements, which would be a serious methodological flaw. Nevertheless, these trends in locus of control applications need to be considered when interpreting the results of studies that are based on this construct. 


\subsection{Applications of the construct}

Having briefly considered the impact of gender and age, it is worth looking at some of the applications of the locus of control construct which are relevant to the studies in this thesis. Thus, this section focuses on aspects of pain, low back pain and health education. There are of course many other applications of this construct which are not addressed here.

Before reviewing these individual application, it is pertinent to consider how the locus of control construct relates to the broader issue of empowerment. Empowerment, addressed in section 3.2, is defined as '... the notion of people having power to take action to control and enhance their own lives, and the processes of enabling them to do so ${ }^{\text {(93) }}$. The links between an internal locus of control and empowerment are obvious, since they both share the notion of people actively taking control and enhancing their own lives. This is particularly pertinent in health issues, where professionals may wish to empower clients to take an active role, and become partners in their health care. One way of achieving this might be by maximizing clients' internal locus of control. This is discussed further in chapter five, when G.Ps give clients an information leaflet with an empowering message that the leaflet will enable them to be in control of their own back pain.

\subsubsection{General health}

When considering the locus of control construct, illness and hospitalization are seen as externalizing events that can be modified by emphasizing self-reliance in health care ${ }^{(61,77)}$. Walding suggests that those who are identified as having an internal locus of control are more likely to cope effectively with their anxiety and thus experience less pain ${ }^{(77)}$. However, such a view assumes that an individual can actually cope with their symptoms and exhibit a degree of control, which may not always be the case. If a person who demonstrates a more internal orientation subsequently feels unable to influence their situation, potentially this could be devastating for them as they may not have previously developed coping mechanisms for dealing with situations that are 'beyond their control'. 


\subsubsection{Information and health promotion}

In 1962 Seeman and Evans reported the findings of a significant piece of research involving white, male clients who were hospitalized with tuberculosis (TB $)^{(94)}$. Clients were asked to complete an Alienation (powerlessness) Scale, which was an early I-E measure. Clients were matched for socio-economic and educational backgrounds, for health and hospital histories. Those with a more internal orientation as determined on the Alienation Scale, knew more about their TB than their matched externals. However, despite this superior level of knowledge, these internal clients expressed beliefs of being significantly less satisfied with the information they had received from health care professionals than their matched counterparts ${ }^{(94)}$. This was an important finding which emphasized the need to try and address clients' expectations and understand their beliefs.

\subsubsection{Pain}

It has been proposed that studying locus of control orientation may be particularly relevant to the experience of persisting pain ${ }^{(79)}$, since clients with chronic pain who have an external locus of control may employ a different set of pain coping strategies to clients with a more internal locus of control ${ }^{(79)}$.

As well as pain perceptions, the locus of control construct has important implications in management of pain. For example, patient-controlled analgesia (P.C.A.), which is sometimes used post-operatively, is an interesting treatment to study as it has great potential to be influenced by the locus of control construct.

P.C.A. approximates a negative 'feedback loop' where a pain stimulus $\rightarrow$ a client response (self-administration of analgesia) $\rightarrow$ subsequent reinforcement (i.e. analgesia) ${ }^{(95)}$. In a study of female clients undergoing abdominal gynaecological procedures, those with an external locus of control (measured on the Multidimensional Health Locus of Control Scale) reported higher levels of pain and greater dissatisfaction with P.C.A., whereas an internal locus of control was predictive of lower pain scores and increased satisfaction ${ }^{(95)}$.

It is not clear from this work whether all clients were similarly instructed in the P.C.A. modality. Presumably they were, and if so, this has important implications for the administration of this treatment, since perhaps those clients with a more external locus of 
control orientation need a more detailed and structured approach to enable them to understand the philosophy of P.C.A. and its application. This 'internality training' might enhance their involvement in the treatment, and thus improve compliance and satisfaction. Such findings would highlight the need for tailoring informed consent and client involvement in treatment regimes according to personality and psychological factors such as locus of control orientation - an interesting concept that clearly warrants further research.

\subsubsection{Chronicity of pain}

Surprisingly few researchers report on the effect of chronicity of symptoms on perceptions of control. Härkäpää et al. report on a sample of clients aged 35-54 years, where the average time since the first symptoms of low back pain was 14.6 years for 289 males and 13.2 years for 170 females $^{(91)}$. In this population, a long duration of back pain was inversely related to an internal locus of control and the authors conclude that the longer the pain had persisted and the more severe it was, the stronger were the feelings of helplessness ${ }^{(91)}$. This is not surprising, since this long after the onset of symptoms, it is unlikely that many clients will demonstrate strong beliefs in health care professionals (powerful others).

\subsubsection{Low back pain}

According to Skevington, a group of people who had attended a back pain clinic for treatment 4-5 years ago (but were no longer being treated as outpatients), and who were still in pain with unclear physical diagnoses, believed the world was controlled more by chance than powerful others ${ }^{(96)}$. This contrasts with Skevington's earlier comment that

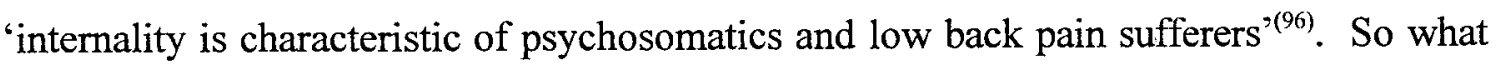
appears to be happening? Perhaps as the chronic low back pain persists, the client's locus of control orientation becomes more external (which is consistent with the earlier ideas $^{(61,77)}$ about illness being an externalizing event. However, if the 'powerful others' have been unsuccessful in diagnosing or treating their symptoms, clients then may move towards a chance orientation. This may then result in the strong feelings of helplessness that Härkäpää et al. describe ${ }^{(91)}$. 


\subsubsection{Treatment for low back pain}

In Härkäpää et al.'s study of clients with chronic and recurrent low back pain, not surprisingly, back surgery was related to stronger external beliefs. This might be expected, because as the authors propose, those with stronger beliefs in medical expertise (i.e. powerful others) are 'probably more willing to submit to back surgery as a treatment of their problem ${ }^{\prime(91)}$. However, what is not known is how such clients' views and locus of control orientations were influenced by the experience of back surgery: As Härkäpää et al. state, it is possible that in the course of hospital treatment, external beliefs are reinforced, and higher externality is the result of an iatrogenic process ${ }^{(91)}$.

\subsection{Ethical considerations}

It is interesting that despite the hundreds of papers that discuss aspects of locus of control, few consider the ethical implications that are inherent within this construct. For example, clients have rights to make informed decisions about their health care, which means that they have the potential to make 'wrong decisions' as perceived by health care professionals. That is the client's choice, but if they do make choices which do not coincide with what the health care professionals perceive as appropriate, then clearly this could be difficult, particularly for the professional who is likely to feel the tension between giving the client autonomy to make an informed decision, whilst simultaneously fulfilling their own obligations and duties of care.

In many ways, the health care professional will probably find this situation more difficult than the client, since according to Montbriand and Liang, it would appear that 'patients believe they have control at all times and do not even consider any of their health care actions as non-compliant ${ }^{(97)}$. Clearly research and treatment programmes involving the locus of control construct need to give this issue due consideration, and enable health care professionals to develop this uncomfortable and apparently conflicting role.

\subsection{Limitations of the construct}

Having looked at some of the applications of the locus of control construct, it is essential to explore the limitations of this work. 
To date the locus of control construct has not lived up to its promise of practical utility $^{(69)}$. It was originally expected to be a useful predictor, although the predictive value of locus of control alone has not been shown to be strong ${ }^{(69)}$.

There is much literature that reports findings of research with the locus of control construct as disappointing, but what is not clear is why the construct has not lived up to its expectations. Is it the construct itself that is where the problem lies? Is it the measurement tools that attempt to measure it? Or does the problem lie with the quality of the research undertaken to test the construct? Each of these will now be considered.

\subsubsection{Limitation of the theory}

There is much debate over whether the locus of control construct has increased our understanding and ability to explain, predict and control events. ${ }^{(61)}$.

The literature on the construct has developed considerably in the late 1970s and 1980s, although in the last decade, this rate of progress has slowed considerably, and criticism of the construct has become more widespread. Whilst on paper, the locus of control construct seems both important and relevant, other predictors, such as health values and beliefs appear to have a more important impact ${ }^{(69)}$.

Waddell and Main suggest that clients' beliefs and ability to cope, play an important role in low back pain and disability ${ }^{(98)}$. They emphasize the importance of overcoming harmful beliefs, improving coping abilities and establishing clients' beliefs about treatment ${ }^{(98)}$, concluding that 'We should have more faith in the power of human thought and in our patients' own capabilities, ${ }^{\text {(98) }}$.

Wallston himself, who has contributed so much to the locus of control literature has challenged the whole notion of locus of control in a paper aptly titled 'Hocus-pocus, the focus isn't strictly on locus ${ }^{\text {(73) }}$. In this paper, he reiterates all the facets of the social learning theory i.e. that it is 'an expectancy-value theory, not simply a theory about expectancies'. This means that besides the expectancy that a person's behaviour will lead to a particular outcome in that situation, the value of the outcome to the person must also be considered, - which is the part, so often ignored ${ }^{(73)}$.

Although as Wallston reflects, the internal-external distinction 'struck the right responsive note for the times', ideas have changed and the body of knowledge has 
progressed. At the start of the thesis work, working with the construct was timely and relevant. In recent years, the locus of control construct has been labelled 'a relatively small portion of the larger and more important construct, perceived control over health $^{\text {(73) }}$. Even so, it suffices to say that the construct has its limitations.

One criticism with the construct is that it is difficult to know the degree to which respondents are actually expressing locus of control beliefs, and to what extent these are coloured by respondents' attempts to present themselves in a favourable light ${ }^{(71)}$. Strickland argues this issue may be particularly important in situations with strong social demand characteristics, such as treatment programmes for people involved with substance abuse, where for example, responses may influence length of stay ${ }^{(71)}$.

The preceding discussion has identified some potentially serious limitations that need to be addressed, before the real value of the locus of control construct can be established, however large a part it contributes to the 'perceived control over health' literature.

\subsubsection{Limitations of the measurement}

Apart from the limitations within the locus of control theory, it is likely that there are considerable flaws in the measurement scales that are used to attempt to measure this construct. These issues are discussed in detail in chapter four, which includes a critical review of outcome measures for measuring control in clients with acute low back pain.

\subsubsection{Limitations of the research}

It has been said that the problem may be in the research itself, not in the construct ${ }^{(69)}$. However, it is highly likely that problems exist with both. For example, researchers have failed to consider the multidimensional nature of the construct, and the value of the reinforcer is frequently ignored ${ }^{(69)}$. In addition, we know precious little about how people select a coping procedure and how they decide if it merits long-term commitment. As Leventhal et al. say, 'the reasons for this ignorance are simple: the literature has been dominated by factor analytic approaches that attempt to generate factors describing how people cope with their emotions and ignore how people go about selecting procedures for problem management, how they define the efficacy of particular procedures and the dimensions along which they generalize in applying old procedures to new problems ${ }^{\text {(64) }}$. 
In addition to the inaccuracies in the way the construct is used, there are many practical problems that have hindered research using the locus of control construct, such as small sample sizes ${ }^{(69)}$. Furthermore, most studies are correlational in design ${ }^{(69)}$ and there is a need for more experimental studies ${ }^{(61)}$, since 'the focus is not on manipulating the individual's locus of control and examining the effects on behaviour, but rather on manipulating behaviours and examining the effects on locus of control ${ }^{(69)}$. Having considered the limitations in study design, then the quality of the research is diminished by using instruments that suffer from reliability and validity problems ${ }^{(69)}$, as discussed in chapter four.

Although these limitations need to be considered, few can be addressed completely satisfactorily when researching with this construct, which has slowed the progress of developing the evidence-base for this work.

\subsection{The way forward}

Surprising, from the vast amount of published literature which addresses aspects of acute low back pain, and all that has been written about aspects of control, the number of studies that look at both acute low back pain and control issues is minimal.

One possible reason for this is that much of the back pain literature is published in the 'medical' archives, whereas the control issues form much of the 'social science' literature, and to date, relatively few efforts have been made to cross these artificial boundaries which was discussed in chapter one. It is for positive reasons that these links need to be made between these two fields, and not because of negative aspects, such as dissatisfaction with the traditional medical model.

There is much to be gained from amalgamating medical and social science literature. It may be that the two fields are explored for their similarities. For example, Leventhal identified that illness beliefs are structured around five themes: identity (what is it?); time-line (how long will it last?); cause (what caused it?); consequences (how will it affect me?); cure / control (can it be controlled or cured?) ${ }^{(99)}$. Not surprisingly, these overlap with the four main aspects of the medical model: i.e. recognizing patterns of illness behaviour (signs \& symptoms); inferring underlying pathology (diagnosis); relating physical therapy to underlying pathology (treatment); and the expectation of the 
illness behaviour to improve (cure $)^{(100)}$. It may be that the two fields of medical and social science literature are explored, not for their similarities, but for their differences, such as the way information is imparted to an individual.

The work in this thesis spans both the medical and social science literature. It attempts to take the reductionist diagnosis of acute low back pain and explore it from a psychosocial perspective. Although this is a liberating prospect, it also liberates all the limitations that are inherent in both fields of literature, and magnifies these too, further adding to the challenge of studying acute low back pain.

\subsection{Summary of the chapter}

- For the purpose of this thesis, acute low back pain was defined as an area bounded by the $12^{\text {th }}$ thoracic vertebra and $12^{\text {th }}$ ribs superiorly, gluteal folds inferiorly and contours of the trunk laterally. The duration of pain, was defined by a client taking at least three days at home (off work) or equivalent, if they were not employed.

- Despite the rapid, natural history of resolution for the majority of back pain episodes, low back pain is costly, both to the client and to society. These costs are not just financial, sometimes involving considerable life changes, such as loss of employment and self-esteem.

- Clients' perceived control over their health is a vitally important concept. As yet, it cannot be easily defined and measured, but it is a developing concept, which has important implications for any research involving low back pain, whether it is from a 'medical' or 'social science' perspective. If clients perceive themselves as able to influence their symptoms, and hence exert a degree of control, this can have important physical and psychological benefits, in their road to recovery. 


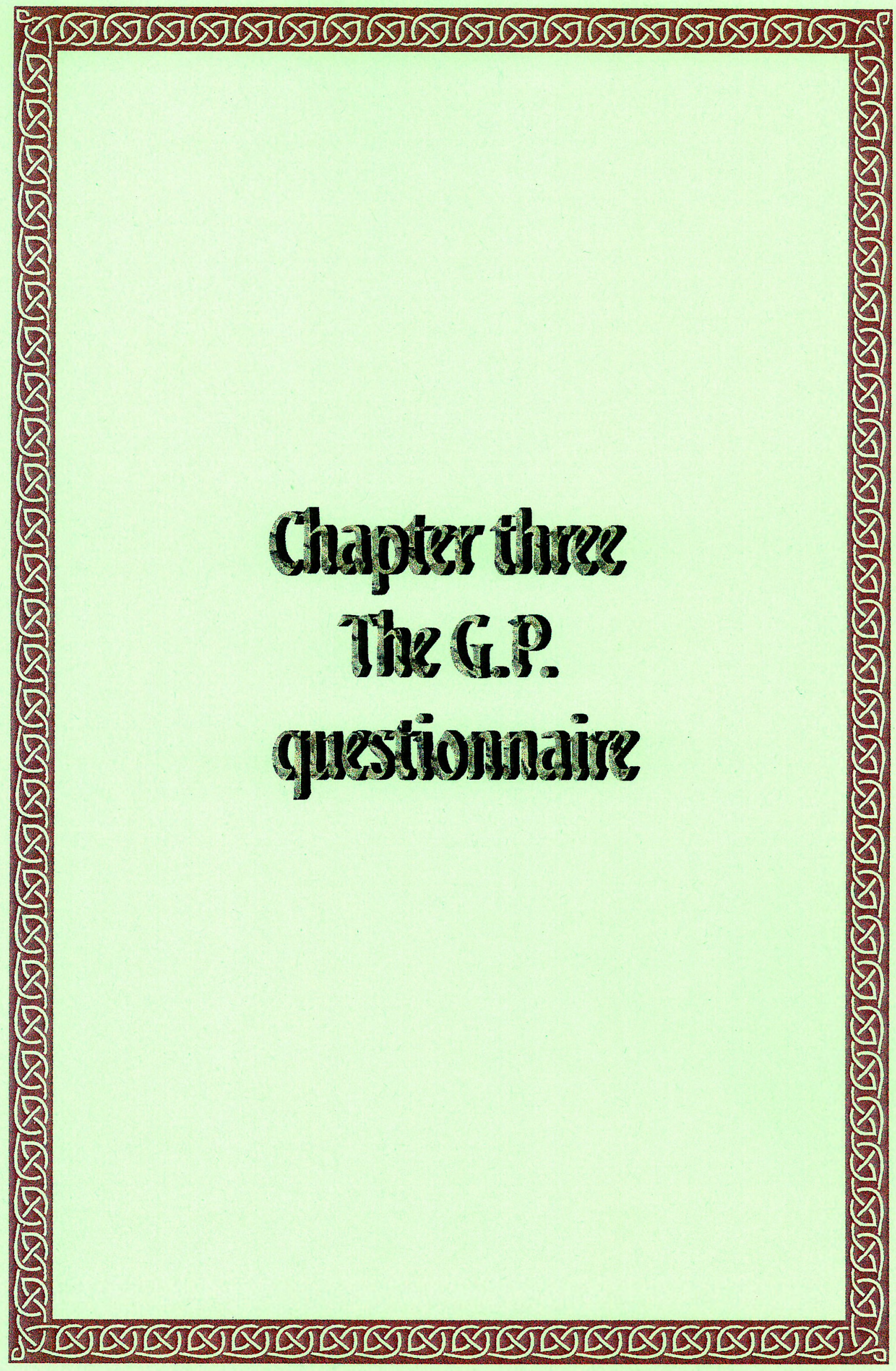




\section{Chapter three: The G.P. Questionnaire}

Chapter two has shown that acute low back pain is a common symptom. In attempting to understand more about its management, in 1993 we obtained funding to develop and test the effectiveness of a leaflet for clients, and produce a diagnostic guide for G.Ps to assist them in their clinical assessment. However, before any work could be done on designing the leaflet or diagnostic guide, it was first necessary to establish the current strategies used by G.Ps for the management of acute low back pain. (This was prior to the publication of the national guidelines determined by the Clinical Standards Advisory Group report ${ }^{(1)}$.)

This chapter describes the development of a questionnaire to establish these strategies, and explores why consulting a G.P. has considerable influence on whether (or not) clients feel empowered, by the actions or advice of their doctor, to manage their back pain. As Deyo states ${ }^{(2)}$ 'Back pain's power to inflict misery is great, but that power is usually transient'. The first, and often only, health professional consulted is usually the G.P. whose behaviour therefore, is crucial in the empowerment process.

\subsection{Acute back pain in general practice}

Before considering why people consult their G.P. with back pain, it is worth establishing how often this occurs. Not everyone with back pain consults their G.P. - indeed, Cherkin et al. ${ }^{(3)}$ state that most episodes of low back pain do not result in visits to health professionals, although they provide no evidence to support this claim. Likewise this issue, although acknowledged in the C.S.A.G. report, is not quantified ${ }^{(1)}$. Walsh however, has estimated that between $40 \%$ and $75 \%$ of those who have low back pain, visit a doctor ${ }^{(4)}$.

It is estimated that in Britain, there are approximately 14-15 million G.P. consultations each year for back pain ${ }^{(1)}$. In the 1993 Office of Population Censuses and Survey ${ }^{(5)}$, it was reported that $16.0 \%$ of the adult population consulted their G.P. with back pain and so crudely, a G.P. serving an average population of around 1900 clients, (range 1700- 
$2100)^{\dagger}$, might expect to see about 300 clients each year presenting with back pain. This estimate will naturally include some people presenting with recurrent episodes of pain, some with chronic back pain, whilst others present with a new episode of acute back pain. Nevertheless, this represents a significant proportion of G.Ps' workload - some 7 or 8 people every working week, for every doctor in the country.

\subsubsection{Why do people consult their G.P?}

According to Ingham and Miller, the most obvious reason for a person to visit their G.P. is that they believe they have a health problem ${ }^{(6)}$. As Cameron et al. ${ }^{(7)}$ state, 'The opportunity to disclose one's concerns, to receive reassurances that one's symptoms are benign, and to receive treatments makes seeking medical care a comforting choice'. Therefore, not surprisingly, consultations with a G.P. (as opposed to other health professionals) are the most common encounters in primary care ${ }^{(8)}$.

So why do some people consult their G.P. while others try over-the-counter medications, seek natural remedies, or use the support of friends and family? Perhaps it relates to clients' expectations: - some may seek attention, a prescription, or an examination and reassurances that nothing serious is wrong; some may see their G.P. as a gate-keeper to further referrals, such as a consultant or diagnostic tests; some may seek their G.P. expecting information and advice; whilst others may have expectations of actual treatment, such as acupuncture or manipulation. These expectations will be influenced by previous experiences (either personal or involving friends or family). Occasionally, clients may consult a G.P. to ask their opinion prior to self-referring to complementary therapists such as homeopaths or faith healers, or to request contact details for any such practitioners.

Some clients may consult their G.P. with the expectation of gaining a label for their symptom, which may also result in a sickness certificate, or which may be perceived as endorsing a claim e.g. for medico-legal purposes. 'Legitimation' of illness, either for work purposes or to family and friends has been shown to be an important factor in attending the doctor ${ }^{(9)}$. Although this finding was in a randomized trial of clients with

\footnotetext{
+ Source: Southampton and South West Hants Health Authority (personal communication) 1998
} 
sore throats, it would seem likely that this could be an important factor for clients consulting a G.P. with acute low back pain, particularly because there are often no visible external signs that a person is experiencing back pain. It may be that individuals are seeking permission to say to their employer and / or family that they need to reduce their workload, at least temporarily.

Overall the role played by social and demographic factors is minor compared with that of symptom severity ${ }^{(6)}$, although amongst these influences, 'the most firmly established factors in the literature include sex differences', according to Briscoe ${ }^{(10)}$.

It is reported that women generally have more contacts (for all causes) with their doctor than men, particularly associated with milder forms of illness and disability ${ }^{(10)}$. However, this is disputed for low back pain, where the sex ratio for consultations is closer to one ${ }^{(4)}$. When it was reported that men consult less often, it was claimed that they have a marked tendency to consult when in need of a medical certificate ${ }^{(10)}$. Briscoe ${ }^{(10)}$ goes further to 'suggest that women are psychologically predisposed to make use of G.Ps more effectively than men', but did not expand on this claim. Clearly this is a controversial topic, which is worthy of further research.

The literature also suggests that marital status also influences a person's likelihood of consulting a doctor ${ }^{(10)}$, for example widowed, separated or divorced women are reported to be 'more likely to consult when compared to other groups who have symptoms of identical severity $^{(6)}$, although the authors do not specify who the people were in the other groups. Thus it is impossible to establish whether this increase in frequency of consultation is related to age, living alone or with others, social networks etc. and hence the original claim about marital status lacks substantiation.

In addition to personal factors affecting consultation rates, environmental factors have also been reported. Walsh et al. describe geographical variation in rates of general practice consultation for low back pain in Britain ${ }^{(11)}$. They undertook a cross-sectional survey of seven British towns and one rural district, although the authors do not report how the locations were chosen. The geographical differences in overall lifetime and oneyear period prevalence were small, but there were considerable variations in the threshold for consulting a G.P. for low back pain within the last year, ranging from $8.3 \%$ in St. Austell and $8.4 \%$ in Dorking, to $18.3 \%$ in Arbroath and $23.5 \%$ in Peterlee ${ }^{(11)}$. After 
allowing for age, sex, social class and disability, people with low back pain in the northern towns of Peterlee and Arbroath were three to four times more likely to seek advice from their G.P. than those in St. Austell and Dorking. The authors highlight possible differences in the perceived benefits of medical consultation or in the accessibility of alternative sources of advice such as chiropractors and acupuncturists ${ }^{(1)}$. It would have been interesting to compare social networks and influences in these different locations to see whether there were any variations, but unfortunately such psychosocial factors were not considered.

Clearly the decision whether to consult a G.P. with medical symptoms such as acute low back pain is both complex and multi-factorial.

\subsection{Why empower?}

Having considered whether people do consult their G.P. with acute low back pain (and why), the next stage was to explore what happens at the initial consultation. The G.P. is able to exert considerable influence on whether (or not) clients feel enabled to take an active role in their own health care - i.e. a process of empowerment.

As an example, a G.P. may instruct a client in how to ease their symptoms, prescribe medication and tell the client when to return to work and whether/ when to re-attend the surgery. In such circumstances, the client is likely to take a relatively passive role in their recovery. If, by contrast, the G.P. enters into a process of negotiation, discussing pertinent lifestyle factors and enables the client to make informed choices about their management, how and when to return to work etc., the client is thus empowered and able to take a relatively active role. So, empowerment is relevant to primary care and it is important to consider what impact it may have on client care.

In chapter two, empowerment was described as '... the notion of people having power to take action to control and enhance their own lives and the processes of enabling them to do so'(12). It implies a redistribution of power in favour of the client with an emphasis on their independence ${ }^{(13)}$. However, opinion is divided on the mechanics of this issue. One group of general practitioners state that 'The empowering process does not result in the patient gaining power while the doctor loses it, but as the patient is empowered by the 
doctor, so the doctor is equally empowered by the ability to care, support and assist the patient $^{{ }^{(14)}}$. They describe the empowerment process as a symbiosis ${ }^{(14)}$.

This idealistic view is not shared by Taylor et al. who suggest that 'Empowerment involves giving more power to users over decisions and hence it probably involves taking power away from service providers ${ }^{\text {(in } 13)}$. Both of these opposing views apparently ignore what Clifton describes as the '... basic point of the empowerment movement that the professional has no right to control the client in the first place ${ }^{\text {(15) }}$. In addition, it does not automatically follow that empowering clients is beneficial. There are some clients who '... don't want to be empowered to improve their own health ... (and) who don't want to make the choices we tell them are good for them ${ }^{(15)}$. It is important that such clients are not coerced into the empowerment process.

From this controversy, it can be deduced that empowerment is a mutual process and both the doctor and client must want to achieve $i^{(14)}$. Each needs knowledge, communication skills and confidence ${ }^{(14)}$. Should the client wish to be empowered, possible benefits might include more active involvement in their own health care ${ }^{(16)}$, and the possibility of many more clients being treated closer to home with self-directed interventions ${ }^{(16)}$. Troop et al. propose that this will free clinicians' time and skill to focus on the more severely ill clients ${ }^{(16)}$, thus channelling resources, which will presumably benefit the clinician and service as a whole.

Client education is a good example of empowerment as it is a process where power is handed back to the client ${ }^{(17)}$. It has been argued that clients 'have a right to know about all aspects of their illness' and that this knowledge must be the foundation for any therapeutic alliance between health professionals and clients ${ }^{(18)}$.

In a small, but in-depth, qualitative study of clients with low back pain in general practice, Skelton et al. found that although some general practitioners recognized the importance of client education, they blamed clients for 'its assumed failure as a management strategy', perceiving that clients failed to retain information given to them in a consultation, and that they lacked motivation to change their behaviour and take responsibility for their back pain ${ }^{(19)}$. Interestingly, in the same study, when clients were asked their views, they considered the information they were given by their doctor to be '... both academic and theoretical in its detachment from specific real-life situations ${ }^{(19)}$. 
The one-way flow of information from G.Ps (as 'experts') to clients (as 'passive recipients of knowledge') encouraged client dependency and discouraged the development of self-reliant attitudes ${ }^{(19)}$. Evidently, both relevant content and an appropriate mode of delivery of client education are vitally important - one, without the other, is useless.

Clearly, G.Ps have tremendous scope to empower, or indeed disempower, their clients by what they say, how they say it, and any action they may take resulting from the consultation. However, at the start of the work in this thesis, little was reported in the literature about how G.Ps empower clients with acute low back pain and the strategies they adopt for managing this symptom.

\subsection{The pilot G.P. questionnaire}

In order to determine the current strategies for managing clients with acute low back pain, the research team (consisting of a consultant rheumatologist, research physiotherapist, general practitioner, physiotherapy manager, lecturer in physiotherapy and statistician), developed a pilot postal questionnaire, based on our clinical experience.

Throughout this work, we chose to use the term 'patients' rather than 'clients', since in previous correspondence with some G.Ps, using the term 'clients' was viewed unfavourably. In this chapter, the terms are used interchangeably, depending whether the context involves direct G.P. contact.

\subsubsection{Methods}

The $3 \frac{1}{2}$ page G.P. pilot postal questionnaire, printed on 'chamois' coloured paper to distinguish it from the many other white documents likely to be on a doctor's desk, contained 39 questions. It covered many aspects of back pain consultations including: details of the assessment; advice given about everyday activities (the categories chosen were modelled on existing client-information leaflets and clinical experience); treatment strategies; referrals for $\mathrm{x}$-rays and consultations; and demographic information about the G.Ps themselves (see appendix 3.1). All aspects of a client consultation were considered, and no weighting of individual questions was used. This was because the purpose of the pilot questionnaire was to collect information from the G.Ps themselves about how they 
manage clients with this symptom, i.e. adding detail to our basic questionnaire, and ensuring that we were asking the 'right' questions. It was anticipated that the questionnaire would take $10-15$ minutes to complete.

The question styles varied including questions that were dichotomous, forced-choice, open, estimated percentages, a visual analogue scale, and two questions ascertaining demographic details about the G.P. population.

\subsubsection{The G.P. population}

The chosen pilot population was 31 G.Ps in Salisbury, i.e. those in city-centre practices, identified from a Family Health Services Authority (F.H.S.A.) practitioner listing. This avoided using the sample that would be approached in the main study, (Southampton and the New Forest).

It was assumed that there were no differences in the management of acute low back pain between the area chosen for the pilot and final G.P. surveys since both sites were in the same health region and had similar geographical location (which was considered important in view of the findings discussed in section 3.1). The similarities between the populations chosen for the pilot and final study (i.e. predominantly urban, in the same health region, in a similar general location, with comparable numbers of G.Ps in the practices) outweighed the differences (such as size of the city, precise location) and thus this assumption was made. Ideally, it would have been preferable to use a small sample of the final study population for the pilot study in case this assumption was invalid, but this was not feasible, due to risk of contamination and a desire to maximize the number of G.Ps available for the final survey. Demographic characteristics of the pilot G.P. population are discussed in 3.3 .4

\subsubsection{Procedures}

Practitioners were approached by letter and asked to give their ideas about 'managing acute back pain (first 3 weeks, severe, new episode)' using the pilot questionnaire. The responses were coded for confidentiality, to enable follow-up reminders to be sent. A stamped addressed envelope was included for their reply. Non-responders were followed-up once, by letter, at two weeks. 
In order to establish test-retest reliability for the questionnaire, it was re-mailed to the same 31 G.Ps two weeks later, a time-scale deemed appropriate by Streiner and Norman ${ }^{(20)}$, with a request to complete it a second time and evaluate the questionnaire using a brief evaluation form (see appendix 3.2). Again, non-responders were followedup once by letter two weeks after the second mailing.

\subsubsection{Results of the G.P. pilot study}

From this pilot group of 31 G.Ps, 22 returned the first copy of the questionnaire (response rate $=71 \%$ ). The demographic characteristics of the pilot population are summarized in table 3.1 .

\section{Table 3.1: Demographic characteristics of the pilot G.P. population}

Results summarizing the demographic characteristics of the G.Ps in Salisbury who responded to the pilot questionnaire $(n=20$, as two failed to complete this section):

\begin{tabular}{|l|l|l||}
\hline $\begin{array}{l}\text { How many years have you been } \\
\text { practising as a G.P? }\end{array}$ & $\begin{array}{l}\text { range }=3-32 \text { years } \\
\text { mean }=14.5 \text { years } \\
\text { median }=13.5 \text { years }\end{array}$ \\
\hline $\begin{array}{l}\text { Have you completed any post-graduate } \\
\text { courses on the management of acute low } \\
\text { back pain? }\end{array}$ & $\begin{aligned} & \text { yes }=7 \text { duration range }=1-10 \text { days } \\
& \text { mean duration }=5.1 \text { days } \\
& \text { median duration }=5 \text { days }\end{aligned}$ \\
\hline
\end{tabular}

In order to investigate whether the duration of practise influences whether G.Ps have completed any relevant post-graduate training courses, a chi-squared test can be done if the duration of practising as a G.P. is analysed as a binary variable (i.e. $<14.5$ years [the mean] or $\geq 14.5$ years). The result $\left(\chi^{2}=0.449\right.$ with $\left.\mathrm{df}=1\right)$, is not significant. Thus it does not follow that G.Ps in the pilot study who have been in general practice longer are more likely to have undertaken post-graduate training in the management of acute low back pain, than their more recently qualified colleagues.

Of course there are other ways of keeping abreast of new developments within back pain management besides attending post-graduate courses, such as reading journals and research reports, but it is particularly difficult for a G.P. to keep abreast of recent advances in all aspects of general practice medicine. 
Having established a few details about the practitioners themselves, it was necessary to ascertain how many clients the G.Ps estimated they saw per month with acute low back pain, since this information would be useful in understanding the relevance of back pain to G.Ps in preparation for the main survey, and for considering potential recruitment rates for the 'Back Home' study. The results are summarized in table 3.2.

\section{Table 3.2: G.Ps' estimates of monthly acute back pain consultations}

Results from the pilot study question requesting G.Ps to estimate the number of patients they see per month with acute low back pain defined as 'first 3 weeks, severe, new episode':

\begin{tabular}{|l|c|c|c|c|c|c|}
\hline Range & 0 & $1-5$ & $6-10$ & $11-15$ & $16-20$ & $>20$ \\
\hline Seen per month & 0 & 3 & 10 & 7 & 0 & 1 \\
\hline
\end{tabular}

In addition to these estimates, one G.P. included a more detailed account of their consultation pattern over the previous year ${ }^{(21)}$ and his one-year figures were:

\begin{tabular}{|lcl|}
\hline All back pain symptoms & 517 & (=43 per month) \\
Back pain alone & 384 & $(=32$ per month $)$ \\
Back pain + sciatica & 77 & \\
Referred for x-ray & 41 \\
New acute episodes & $60 \%$ \\
Recurrent episodes & $20 \%$ \\
\hline
\end{tabular}

This analysis was undertaken without a specific definition of what constitutes acute low back pain, which therefore limits these findings. Presumably 'all back pain symptoms' includes those of non-musculoskeletal origin, e.g. renal disease, viruses etc., and involves multiple body areas e.g. inflammatory arthropathies. Nevertheless, the incidence of 'back pain alone' compares well to the estimates cited in section 3.1.

As the purpose of the pilot study was to inform the final questionnaire, the only other results from the G.P. pilot questionnaire which will be discussed in this chapter are the danger signs question (see 3.4.1), and the issue of referral for x-ray (see 3.4.3). 


\subsubsection{Reliability of the pilot questionnaire}

The response rate for the second mailing $=52 \%(\mathrm{n}=16)$, and overall, the reliability of the questions was variable. At best, test-retest reliability was $100 \%$ (for giving advice about lifting, and testing reflexes in a first examination), and at worst, (when estimating percentages of patients referred to see a rheumatologist or physiotherapist), only four G.Ps gave similar answers (one estimate even went from $10 \%$ to $98 \%$ in two weeks). Clearly the question format was important.

Generally, the questions demonstrating poorest reliability were those with an open format, e.g. the danger signs question, where reliability could be tested in only 11 respondents $^{(22)}$, or those questions which involved estimating percentages (with the exception of estimating percentages of patients who are referred for an x-ray, where no repeated answer varied by more than 10\%). Estimates of referrals to other health professionals were found to be less reliable than estimates for $x$-ray referrals, for example only four G.Ps accurately repeated their estimates of referrals to a rheumatologist, whereas others differed by $20 \%$ and one even differed by $80 \%$.

It was not just these questions which were unreliable. Surprising, even a question such as 'How many years have you been practising as a G.P?', which might be perceived as relatively factual, resulted in four responses differing by two or more years (two differed by two years, and two responses by three years). In three of the four cases, the higher estimate was made in the first response to the questionnaire. This suggests that responses were not carefully considered. Changes made to the final questionnaire following the pilot are discussed in section 3.4.5.

\subsubsection{Evaluation of the pilot questionnaire}

It was important to ascertain their opinions of the questionnaire itself, and so participants were also asked to complete a brief evaluation form. This consisted of six questions with a four point Likert scale response, one question asking whether any issues were omitted, details of the time taken to complete the G.P. questionnaire, and space for any additional comments (see appendix 3.2). The evaluation form was based on the 'Tell us what you think' format devised by Krilyk and Eyles ${ }^{(23)}$. Seventeen responses were received, summarized in table 3.3. 


\section{Table 3.3: Evaluation of the pilot questionnaire}

Results from the forms evaluating the G.P. pilot questionnaire, completed by 17 G.Ps .

\begin{tabular}{|c|c|c|c|c|c|}
\hline & $\begin{array}{c}\text { Strongly } \\
\text { agree }\end{array}$ & Agree & Disagree & $\begin{array}{l}\text { Strongly } \\
\text { disagree }\end{array}$ & $\begin{array}{l}\text { Omitted } \\
\text { question }\end{array}$ \\
\hline Easy to follow & 6 & 11 & 0 & 0 & 0 \\
\hline Relevant to my current practise & 4 & 12 & 1 & 0 & 0 \\
\hline Well constructed & 2 & 11 & 2 & 0 & 2 \\
\hline Too time consuming & 2 & 2 & 11 & 2 & 0 \\
\hline Easy to complete & 2 & 15 & 0 & 0 & 0 \\
\hline Too detailed & 1 & 2 & 12 & 2 & 0 \\
\hline
\end{tabular}

From the results, the mean reported time taken to complete the pilot questionnaire was 8.2 minutes (range $3-12$ minutes).

It can be seen that the majority of G.Ps who completed the evaluation form found the pilot questionnaire easy to follow, relevant to their clinical practise, well constructed, easy to complete and not too time consuming. However, this evaluation form was relatively crude and therefore the comments were particularly important to consider, especially since it is likely that the G.Ps who evaluated the questionnaire had the most polarized views.

Eight practitioners considered that the questionnaire covered all aspects of their current management of acute low back pain, whilst eight did not, and one omitted this question. A total of 11 issues were identified as 'omissions': epidurals; acupressure / acupuncture; manipulation; home visits; 'recurrence in vulnerable patients'; 'work implications for chronic sufferers'; 'malingerers'; trigger factors; 'ergonomic advice and long-term management'; rectal examination; and blood tests. Some of these issues, such as acupuncture and manipulation, were considered important aspects of G.P. management of acute low back pain and so were included in the final questionnaire. Meanwhile, other issues were considered more pertinent to chronic low back pain (such as malingering, long-term management) and so were not included. 
Among the ten comments made in the 'additional comments' section were two comments relating to the questionnaire construction (from the two G.Ps who did not consider the questionnaire to be well constructed):

\section{'Difficult to answer questions about \% of patients receiving drugs / referrals - I doubt the reliability of the replies' \\ 'Confused as to whether acute localized back pain or acute root irritation was being assessed'.}

Both comments were substantiated by the pilot study findings, and so amendments were made to the final questionnaire (see 3.4.5).

There was a range of other 'additional comments', both positive and negative. Examples include:

'This is one amongst many similar requests for my time. I have much sympathy with those G.Ps who routinely file such unpaid work requests in the bin!'

'Anything that focuses attention on this problem is for the good. Hospitals are generally disinterested in it'.

It was helpful to have a spectrum of views in preparation for approaching G.Ps with the main questionnaire.

\subsection{Development of the final questionnaire}

Some responses in the pilot questionnaire raised issues and challenged our assumptions about the evidence-base for danger signs (i.e. clinical signs indicating possible serious spinal pathology) and x-ray referrals. Therefore, before the final questionnaire could be used, it needed further developmental work.

\subsubsection{The question of danger signs}

Included in the pilot questionnaire was an open question: 'What signs and symptoms do you consider are "danger signs" for a patient with acute low back pain i.e. those which would necessitate immediate hospital attention?' This question was asked in an open format to elicit free responses, although generally there were few items identified in the 
responses, containing little detail. The 49 responses, (mean 2.2 per G.P.), were assigned to 21 categories. The top 8 responses (i.e. signs or symptoms selected by more than one G.P.) can be seen in table 3.4 .

One difficulty in categorizing data in this way is that it could be argued that "problems with sphincter control' may also be deemed 'bladder / bowel disturbances'. If the sphincter under consideration is either the anal or bladder sphincters (vesicae or urethrae) $)^{(24)}$ then this is true, but this cannot automatically be assumed, and so the categories are treated as discrete entities.

\section{Table 3.4: G.Ps' perceptions of danger signs and symptoms}

Results from the 8 most frequent responses in the pilot questionnaire asking G.Ps to identify what they considered to be danger signs and symptoms for a patient with acute low back pain.

\begin{tabular}{|l|c|}
\hline & Responses \\
\hline Signs/symptoms of bladder/bowel disturbances & 10 \\
\hline Problems with sphincter control & 8 \\
\hline Severe/disabling pain & 6 \\
\hline Gross neurological complications & 3 \\
\hline Obvious root signs & 3 \\
\hline Objective signs & 2 \\
\hline Paralysis & 2 \\
\hline Progressive weakness / loss of reflexes & 2 \\
\hline
\end{tabular}

Having sought opinions from the G.Ps as to what they considered danger signs and symptoms, the next stage was to search the literature to ascertain the reported evidencebase for these signs.

Excluding the presence of these danger signs in people with acute low back pain is perhaps the most important aspect of a G.P. consultation. Thus, following a careful examination, advising a client that tests for serious spinal pathology are negative, can be both reassuring and empowering. It is a fundamental part of clinical assessment, which could significantly affect clients' perceptions of their control of their back pain. 
However, it assumes that the literature about detecting these signs is accurate, disseminated to practitioners and able to be implemented in clinical practise. The first stage is to review the existing literature.

In an attempt to ensure that no major danger signs had been omitted by the pilot G.P. population, the inter-disciplinary research team (comprising clinicians from rheumatology, general practice, physiotherapy), also identified a list of potential danger signs / symptoms, and further opinions were sought from medical colleagues in the Orthopaedics, and Accident and Emergency Departments and the Wessex Neurological Centre, at Southampton University Hospitals Trust. Additional potential signs and symptoms included: extensor plantar response; night pain; and bilateral leg signs. Once this final list of potential danger signs was established, a literature review was then undertaken.

\subsubsection{What evidence is there for danger signs (or 'red flags'?)}

Reviewing the literature revealed a surprisingly poor evidence-base for danger signs. The reported studies were primarily either individual case histories or studies with few subjects, and often lacked clinical details.

From this literature review and further consultation with medical colleagues previously identified, a hierarchy of danger signs was devised, in preparation for the final G.P. questionnaire.

In this hierarchy, some signs, usually reported in the literature to be indicative of serious spinal pathology, we termed 'probable danger signs'. When evidence was more controversial, we termed them 'possible danger signs', and when no little or no evidence could be found, they were deemed 'probably not danger signs': 


\begin{tabular}{|ll|}
\hline 1. 'probable danger signs' & $\begin{array}{l}\text { i.e. extensor plantar response } \\
\text { neurological signs at multiple levels } \\
\text { saddle anaesthesia }\end{array}$ \\
2. 'possible danger signs' & $\begin{array}{l}\text { i.e. [constant] night pain } \\
\text { bilateral leg signs }\end{array}$ \\
3. 'probably not danger signs' & $\begin{array}{l}\text { i.e. severe local back pain } \\
\text { loss of reflex at one level } \\
\text { unilateral sciatic symptoms below the knee. }\end{array}$ \\
\hline
\end{tabular}

This list was not intended to be definitive, but aimed to address symptoms that may be present in the absence of any notable history such as trauma, previous history of carcinoma, systemic steroids etc. Having identified these eight signs and symptoms, the underpinning evidence base is now considered.

\section{'Probable danger signs'}

i) Extensor plantar response: The plantar response has been described as the most important reflex in the body and yet the one most frequently misinterpreted ${ }^{(25)}$. The phenomenon was first described in 1896 by Joseph Babinski, a Polish neurologist, working in a syphilitic ward in Paris ${ }^{(26)}$. He observed that stroking the lateral aspect of the foot facilitated 'dorsiflexion of the big toe in patients with meningovascular syphilis affecting the corticospinal tract ${ }^{(26)}$, and went on to describe a sign, evoked by nociceptive stimulus that consisted of dorsiflexion of the toes and ankle, with flexion at the knee and hip $^{(27)}$. In 1903 Babinski added abduction or fanning of the toes to his original description, but noted that this was not a constant component ${ }^{(27)}$ : Babinski found fanning of the toes occasionally occurred in healthy people, and was often found in patients with early pyramidal tract lesions before the plantar response became extensor ${ }^{(28)}$.

The presence of an extensor response in clinical practise indicates a disturbance in function of the pyramidal system and this notion is supported by clinical and experimental agreement ${ }^{(25)}$. However, the presence of the sign does not necessarily indicate a lesion of the pyramidal tract, and indeed there is some debate over whether the pyramidal tract is responsible for the response or whether other descending fibre systems may be involved ${ }^{(26)}$. Even Babinski is reported to say ${ }^{(i n}$ 29) 'This sign, although it indicates 
the presence of a pyramidal system disturbance, does not denote its severity', and Macgregor ${ }^{(29)}$ comments on his use of the term pyramidal system as opposed to pyramidal tract, although he does not discuss whether this is a translation, (since Babinski's papers were originally written in French).

The extensor plantar response does not give any indication of the degree of damage ${ }^{(28)}$, and may also be found in some groups of people who are not experiencing dysfunction of the pyramidal tract including: people in a coma ${ }^{(25)}$; infants (due to immaturity of the pyramidal tracts) ${ }^{(28)}$; elderly clients with psychiatric disease ${ }^{(30)}$; hypoglycaemia ${ }^{(26,31)}$; post ictally $^{(26)}$; and even in people who are in a very deep sleep ${ }^{(26)}$. If no response is elicited on testing, this may be due to either the client not being relaxed or if they are cold ${ }^{(25)}$. It can also be difficult interpreting the sign if the soles of the feet are particularly tender or sensitive $^{(28)}$.

ii) Neurological signs at multiple levels: The evidence for neurological signs at multiple levels being indicative of likely serious spinal pathology, is based on knowledge of anatomy and pathology. Herniated discs are sometimes cited as mimicking extraosseous spinal tumours ${ }^{(32)}$, and Epstein ${ }^{(\text {in }}{ }^{32)}$ determined that approximately $1 \%$ of clients presenting with symptoms consistent with disc herniation have intraspinal tumours.

Unfortunately many studies reported in the literature are not specific in describing the details of the clinical signs present in their clients. For example Levy et al. report that $30 \%$ of their series of 66 patients with neurofibromas had 'motor dysfunction with weakness', but it is unclear as to the extent of these signs and how they were measured $^{(33)}$. Likewise, Delamarter et al. report a series of 29 clients with 'primary osseous thoracic and lumbar vertebral neoplasms' over a 17 year period, and state that 'sixteen patients had demonstrable neurologic deficits, including motor weakness in eight, sensory changes in six, lower extremity spasticity in six, abnormal deep tendon reflexes in six, and bowel and bladder incontinence in three ${ }^{(34)}$. Such descriptions still omit detail of the levels involved, even though it is clear that for bowel and bladder incontinence, multiple levels will be affected, as the nerves supplying the bladder form the vesical plexus ${ }^{(24)}$. 
Despite the non-specific reports cited, some evidence of neurological signs at multiple levels being indicative of possible serious spinal pathology does exist. Nayernouri describes a series of 20 clients with neurilemomas, where nine clients had 'back pain and bilateral sciatica with signs implicating two or more roots of the cauda equina'(35). Donaldson et al. reviewed a group of 15 clients with 'symptomatic metastatic melanoma to the spine' seven of whom presented with neurological findings: 'Most patients had multiple spinal level involvement with an average of 3.1 levels per patient (range $1-9$ levels) ${ }^{(36)}$.

iii) Saddle anaesthesia: In texts describing clinical examination of the spine, a positive finding of saddle anaesthesia 'makes immediate surgical referral imperative ${ }^{\text {(37) }}$, as an 'urgent decompression may be indicated' ${ }^{(38)}$. Dinning and Schaeffer ${ }^{(39)}$ state that permanent bladder and rectal dysfunction will result if the compression of the cauda equina is not speedily and effectively relieved. In such texts, saddle anaesthesia is described as a feature of 'cauda equina syndrome', (along with loss of rectal sphincter tone, bilateral leg weakness or reflex loss $)^{(40)}$. However, in many case histories of clients with spinal tumours resulting in symptoms of cauda equina compression, saddle anaesthesia is surprisingly rarely reported. Three possible reasons for this may be: i) incomplete records (as many are reported in retrospective series of case histories); ii) because the focus of reporting has been on clinical signs rather than the clients' symptoms; or iii) when details are included, they are nebulous e.g. Levy et al. describe 'bowel or bladder difficulties'(33), giving no indication of the nature of these difficulties. It is often assumed that the only urinary symptom clinicians need to be particularly vigilant for is urinary retention, but Grieve defines 'sphincter problems' as 'loss of control, retention, hesitancy, urgency or a sense of incomplete evacuation ${ }^{(38)}$.

Despite these issues, some evidence that saddle anaesthesia is indicative of serious spinal pathology has been reported, e.g. Matthew and Todd state that 'sensory disturbance in the saddle (perineum and buttocks) region occurs in both intramedullary and extramedullary tumours and invariably accompanies a disturbance in sphincter function'(41). $^{\text {, }}$ 


\section{'Possible danger signs'}

i) (Constant) night pain: If clients describe their pain as 'constant', it is important to establish what happens to the diurnal pain pattern, including at night, because surely, for pain to be described as truly 'constant', this must also include night pain? A number of studies report case histories of clients with spinal tumours, who sometimes describe their pain as being worse at night ${ }^{(34,35)}$. However, night pain may not always be present when serious pathology exists. For example, Boriani et al. report the findings from a series of 30 clients with osteoblastomas of the spine where all 30 clients complained of 'continuous local pain, with only four noting an increase in pain at night' ${ }^{(42)}$. Likewise in nine clients with non-malignant intraspinal tumours at the Texas Back Institute, only three complained of night pain and / or pain that increased in the supine position ${ }^{(32)}$.

Clinicians must establish whether any reported night pain occurs alone or is associated with other signs and symptoms and it is vital to exclude environmental factors such as the client's bed, and pathological processes such as inflammation, arthritis etc. Having eliminated these issues, taking a clear history of painful trigger factors should help determine whether the pain reported is due to a 'mechanical' cause, or whether serious spinal pathology should be suspected.

It is highly probable that night pain (or truly constant pain) constitutes possible serious spinal pathology, and Nayernouri particularly describes 'night pain relieved by walking' as an important sign to watch for $^{(35)}$, as do Matthew and Todd ${ }^{(41)}$. Delamarter et al. conclude that 'constant pain unresponsive to conservative therapy, night pain, and pain enhanced by laying supine, are suggestive of a neoplastic process ${ }^{{ }^{(34)}}$.

ii) Bilateral leg signs: Many studies fail to specify whether reported signs are bilateral. Case reports, although often less generalizable than clinical studies, may yield more detailed information. Guyer et al. describe a case report ${ }^{(32)}$ in which a 33 year old client reported recurrent low back pain and unilateral leg pain but after a long automobile drive, she reported low back pain and bilateral leg pain, then three days later noted 'weakness in her lower legs and urinary hesitancy'. Emergency myelogram revealed a complete block from T11 to L3, and at surgery this was found to be due to an ependymoma ${ }^{(\hat{2} 2)}$. The authors also report a second case this time in a 37 year old client where one of the 
features mentioned was progressive weakness of both legs, which was due to a neurolemmoma ${ }^{(32)}$.

In the light of such evidence, serious spinal pathology could be present in a client presenting with bilateral leg signs, hence its classification as a 'possible danger sign'.

\section{'Probably not danger signs'}

Evidence for loss of reflex at one level or unilateral sciatic symptom below the knee were not reported in any of the case histories or studies reviewed. Hence they were classified as 'probably not danger signs' in our hierarchy, and used to intersperse with the other 'probable' and 'possible' danger signs. Likewise, severe local back pain was also placed in this category, because although it was sometimes described as a feature of the cases presented $^{(32,36)}$, as Delamarter et al. state, it '... is not diagnostic and can be associated with other spinal disorders ${ }^{\text {(34) }}$. Thus, it is the details about the pain that are more important, such as its aggravating and easing factors, diurnal patterns etc.

Therefore, the danger signs question which formed part of the final G.P. questionnaire was amended to read: 'What signs and symptoms do you feel justify immediate hospital advice for a patient with acute low back pain?'

Yes No

a) Severe local back pain

b) Bilateral leg signs

c) Loss of reflex at one level

d) Extensor plantar response

e) Constant night pain

f) Unilateral sciatic symptoms below the knee

g) Saddle anaesthesia

h) Neurological signs at multiple levels

Using such a format required G.Ps to consider each sign or symptom individually and make a simple 'yes / no' decision. The eight items were not arranged in a hierarchical order, to avoid cueing the G.P. responses. 


\subsubsection{The question of $x$-rays}

Like aspects of the client examination, whether or not a G.P. decides to refer a client for an x-ray, can be an important factor in the empowerment process. If the G.P. dictates to a client that they must have an x-ray, this could be perceived as disempowering. However, explaining the advantages and disadvantages of such a referral and reaching a decision in conjunction with the client, could be deemed a more empowering approach. Therefore, in order to help understand whether G.Ps may empower clients with the use of $\mathrm{x}$-rays, it was necessary to explore the underlying reasons why a doctor might refer a client with acute low back pain for an $\mathrm{x}$-ray in the first place.

In the pilot study, G.Ps were asked an open question: 'What criteria do you use when deciding whether x-rays are appropriate for a patient with acute low back pain?' A total of 45 comments were made (mean 2.1 per G.P.), which were assigned to 25 categories. The top 9 responses (i.e. criteria selected by more than one G.P.) can be seen:

\section{Table 3.5: Pilot study G.Ps' top 9 criteria for requesting $x$-rays}

Results from the pilot questionnaire asking G.Ps to identify what criteria they used when deciding whether $\mathrm{x}$-rays are appropriate for a patient with acute low back pain.

\begin{tabular}{|l|c|}
\hline & Responses \\
\hline Worried about other pathology & 5 \\
\hline Past medical history of carcinoma ${ }^{\dagger}$ & 5 \\
\hline Patients' age & $4+2^{\ddagger}$ \\
\hline Persistent pain & 3 \\
\hline History of trauma & 2 \\
\hline When the patient insists! & 2 \\
\hline Objective neurological signs & 2 \\
\hline Recurrent acute episodes & 2 \\
\hline Worried about possibility of spondylolisthesis & 2 \\
\hline
\end{tabular}


Reasons given by individual practitioners for referring a client to have an $\mathrm{x}$-ray included 'medical' reasons, e.g. 'atypical pain', 'chronic generalized $O A$ ', as well as 'social' reasons, e.g. 'trying to satisfy the patients' wants!', 'if doubts!' etc. Such openness, expressed in the 'social' reasons, surprised the research team.

Having sought opinions from the G.Ps as to why they refer clients for x-rays, the next stage was to review the guidelines that exist for clinical practise to see what reasons they advocated for requesting $x$-rays.

\subsubsection{What guidelines are there for referring clients for $x$-rays?}

$\mathrm{X}$-rays of the lumbar spine account for $3 \%$ of all $\mathrm{x}$-rays ${ }^{(1)}$, and they are also used 'extensively' by chiropractors in the private sector ${ }^{(43)}$. In 1995 the Royal College of Radiologists (R.C.R.) revised their guidelines ${ }^{(44)}$ (and again in 1998) ${ }^{(45)}$, in an attempt to reduce the number of routine $x$-rays used in the management of acute low back pain ${ }^{(44)}$, since G.Ps order back $x$-rays in $20 \%$ of patients ${ }^{(1)}$. The most recent guidelines incorporate a greater number of danger signs that justify the use of imaging techniques $^{(45)}$. Specifically the guidelines recommended that no 'routine' $\mathrm{X}$-rays are taken for acute low back pain with no adverse features (danger signs), since 'Acute back pain is usually due to conditions which cannot be diagnosed on plain XR (osteoporotic collapse an exception) ${ }^{(45)}$. It has been estimated that if the R.C.R. guidelines were followed, the number of lumbosacral spine $\mathrm{x}$-rays would be reduced by $50 \%{ }^{(1)}$.

Evans and Richards summarized three reports on the selective use of spinal $x$-rays in investigating low back pain ${ }^{(43)}$, although the R.C.R. guidelines quoted are the 1993 edition $^{(46)}$, not the third edition, revised in 1995. It is also unclear why the C.S.A.G. ${ }^{(1)}$ and Agency for Health Care Policy and Research (A.H.C.P.R.) ${ }^{(47)}$ guidelines were not included. The summary ${ }^{(43)}$ simplified in table 3.6, does have some omissions. For example, Deyo also advocated 'findings suggestive of ankylosing spondylitis' and 'seeking compensation' as reasons for the selective use of spinal x-rays in investigating low back pain ${ }^{(40)}$, which were not included in Evans and Richard's table ${ }^{(43)}$ and the Quebec Task Force also cite recurrent spinal disorders ${ }^{(48)}$. 


\section{Table 3.6: Recommendations on the selective use of spinal $x$-rays in} investigating low back pain (after Evans and Richards 1996) ${ }^{(43)}$

A table showing the differences between the recommendations of three sources regarding the use of spinal $x$-rays in clients with low back pain. In addition to the differences listed, all three sources advocated the use of x-rays with a history of significant trauma, when significant abnormal neurological signs are evident on examination, or if there is a justified suspicion of malignancy or infection on history and examination.

\begin{tabular}{||l|c|c|c||}
\hline \multicolumn{1}{|c|}{ Indication } & \multicolumn{1}{|c|}{$\begin{array}{c}\text { Royal College of } \\
\text { Radiologists }\end{array}$} & $\begin{array}{c}\text { Quebec Task } \\
\text { Force }^{(48)}\end{array}$ & Deyo $^{(40)}$ \\
\hline Age (years) & $\begin{array}{l}>60 \text { years with suspected } \\
\text { vertebral collapse and } \\
\text { 'younger patients' }\end{array}$ & $>50$ or $<20$ & $>50$ or $<20$ \\
\hline $\begin{array}{l}\text { History of intravenous } \\
\text { drug abuse }\end{array}$ & no no & no & yes* \\
\hline Use of corticosteroids & \multicolumn{1}{|c|}{ no } & yes \\
\hline $\begin{array}{l}\text { Period after which } \\
\text { pain has not improved } \\
\text { or is worsening }\end{array}$ & $\begin{array}{l}\text { After 6 weeks in acute pain. } \\
\text { (The C.S.A.G. management } \\
\text { guidelines stipulate 'at } \\
\text { 6-weeks if specifically } \\
\text { indicated') }\end{array}$ & After 4 weeks & $\begin{array}{l}\text { After 2-3 } \\
\text { weeks }\end{array}$ \\
\hline
\end{tabular}

Key: $*=$ Due to risk of spinal infection.

More recently, the Royal College of General Practitioners (R.C.G.P.) Clinical Guidelines for the Management of Acute Low Back Pain ${ }^{(49)}$ stated 'plain X-rays are not recommended for routine evaluation ... within the first month of symptoms unless a red flag is noted on clinical examination'. Plain x-rays are recommended for ruling out fractures if there is any recent significant trauma (any age), recent mild trauma (patient over age 50), history of prolonged steroid use, osteoporosis, or patient over age $70^{(49)}$. The guidelines also advocate plain x-rays in combination with full blood count and erythrocyte sedimentation rate in ruling out tumour or infection in clients with "prior cancer or recent infection, fever over 100F, IV drug abuse, prolonged steroid use, low back pain worse with rest, unexplained weight loss ${ }^{\text {(49) }}($ sic $)$.

So, when a G.P. is consulted by a client with acute low back pain, they need to consider not only the clinical guidelines when deciding whether to refer the client for an x-ray, but also any likely benefit from this $x$-ray, the dangers of this invasive technique, its cost and 
the pressure on the local radiography department. The decision can therefore be complex.

$\mathrm{X}$-rays may be of limited use in acute low back pain. Deyo reports a poor relationship of many abnormal findings to symptoms ${ }^{(40)}$. In addition all soft tissues are radio-translucent and thus are not visible on x-ray. Therefore there seems little point in referring a client for $x$-ray if one of these tissues is the suspected cause of their pain. It is also important to remember that serious pathology can exist in the presence of normal $\mathrm{x}$-rays as it takes time for such disease processes to produce bony destruction. False negative x-rays are common in the early stages of both tumour and infection ${ }^{(1)}$. Indeed Rogers reports that spine bone deposits do not usually show on plain x-ray until $50 \%$ of trabecular bone is damaged $^{(50)}$.

After deciding whether an $\mathrm{x}$-ray will be clinically useful, the value of plain $\mathrm{x}$-rays must then be balanced against the radiation exposure involved ${ }^{(51)}$. The effective dose for the lumbar spine is calculated as 2.4 milliSieverts, which according to the Royal College of Radiologists, is equivalent to 14 months of natural background radiation ${ }^{(44)}$. Every radiation exposure carries a one in 80000 risk per $\mathrm{mSv}$ of inducing a fatal cancer ${ }^{(52)}$, and due to the levels of radiation, Halpin et al. ${ }^{(52)}$ statistically estimate that $\mathrm{x}$-rays of the lumbar spine could cause 19 radiation deaths each year in Britain. This therefore, has ethical implications in gaining informed consent from the client as the potential dangers (however unlikely) should to be stated. There are also responsibilities for the environment, since it is reported that 'Medical sources are by far the largest artificial cause of ionising radiation, representing $12 \%$ of the background radiation in the United Kingdom, over ten times the radiation due to radioactive discharges'(52).

Assuming that the x-ray will be useful and the client has given informed consent (and is aware of the dangers of radiation), the next stage for the G.P. is to decide on the number of views that are required. As many as five views (anterior-posterior (A.P.), lateral, two oblique views and a coned lateral view of the L5/S1 level) have been advocated in some establishments in the U.S.A. ${ }^{(43)}$, although the A.H.C.P.R. guidelines recommend against routine oblique views ${ }^{(47)}$. An A.P. and lateral view remain the views of choice in the United Kingdom (U.K.) ${ }^{(43)}$. Deyo reports that from 2397 consecutive lumbosacral spine examinations, 'In only one case was a diagnosis apparent only on the oblique views', and 
the case was a client with a (benign) osteoid osteoma ${ }^{(40)}$. Restricting the number of views taken will also help minimize the cost and the amount of radiation a client receives, and hence the likelihood of adverse effects such as radiation-induced carcinoma. Even when the number of views has been chosen and the x-rays taken, there is then the added problem that even highly experienced radiologists interpret the same x-rays differently, leading to uncertainty and even inappropriate treatment ${ }^{(2)}$.

According to Papageorgiou et $a$ lin $^{\text {(in } 53)}, 13 \%$ of G.P. consultations result in a radiology

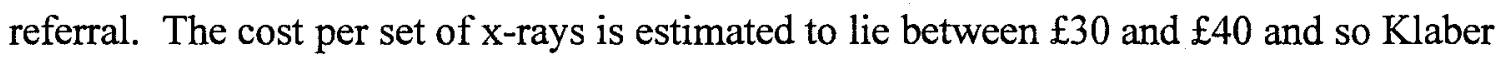
Moffett et al. estimate that the costs of radiographic spinal examination is between $£ 26.7$ million and $£ 60.1$ million annually ${ }^{(53)}$. Note that this figure does include the costs of out-patient radiology too, although the authors argue that the '... double counting ... is probably more than counter balanced by the extra cost of other much more expensive radiological examinations such as Computerized Tomography (CT) and Magnetic Resonance Imaging (MRI) which are sometimes used for non-specific mechanical low back pain ${ }^{\text {(53). }}$. Therefore this figure does not represent the true cost of $\mathrm{x}$-rays alone in primary care. However, it is clear that $x$-rays are costly, invasive and over-prescribed in primary care, suggesting a mismatch between clinical guidelines and actual clinical practise.

\subsubsection{Changes to the questionnaire following the pilot}

From the findings of the G.P. pilot questionnaire in Salisbury, the evaluation forms and the literature reviews, the main questionnaire was then finalized.

The order of questioning in the pilot study was revised to reflect a conventional pattern of a client consultation based on the advice of the G.P. in the research team i.e. assessment questions, advice, sickness certification, any additional investigations, interventions, and plans for following the client up.

Questions were omitted where the management was uncontroversial, such as in the use of analgesia (where only six G.Ps reported using analgesia in fewer than $90 \%$ of patients), or if the management strategy, such as bed rest, depended upon a number of factors such as the severity of pain, presence of muscle spasm or radicular signs ${ }^{(22)}$. 
Apart from the changes to the danger signs and x-ray questions already discussed, other amendments were made to the final questionnaire including:

- clarifying whether G.Ps give any specific advice regarding 'exercise', by dividing 'exercise' into 'specific back exercises' and 'general fitness'

- identifying sickness certification in two categories depending on whether the client presented with 'back pain alone' (somatic pain) or 'root signs' (radicular pain)

- adding a request for information about surgery intervals (i.e. duration of appointment time per client)

- adding questions about acupuncture and manipulation treatments actually performed by the G.P.

- using a four point Likert scale to identify frequency of empowering clients to take responsibility for their follow-up appointment(s)

- removing unreliable questions e.g: estimated percentages of referrals to other health professionals; description of prognosis; and estimates of the number of clients seen each month presenting with acute low back pain

- removing the question asking how G.Ps would respond if people ask how long it will take to recover. The most common response was 'it depends' (x5). Other responses included 'I do not know', 'unpredictable but mostly soon', and various unreliable specified times, from one-day to six weeks duration.

These modifications were discussed and agreed with a group of three G.Ps who were particularly interested in the study and who had already completed the questionnaires twice, to ensure face validity was sought from G.Ps (similar to the target population).

\subsubsection{Validity of the questionnaire}

It was only possible to establish face validity for the final questionnaire from the G.Ps participating in the pilot study and the multi-disciplinary research team - a pragmatic situation rather than an ideal one. This was because in 1993, there were no 'gold standard' clinical guidelines in the U.K. for establishing general practice management of 
acute low back pain, and therefore no measures of criterion validity (discussed further in section 4.6). However, a total of 28 people contributed to the face validity of the final questionnaire (the 22 G.Ps from the pilot study plus the six research team members), resulting in its final format (see appendix 3.3).

\subsubsection{The final G.P. questionnaire}

Following these revisions, the final questionnaire consisted of a mixture of 14 open, nominal, ordinal and ratio questions. The issues covered included: the use of leaflets; aspects of routine acute low back pain assessment; what G.Ps consider to be danger signs (indicating possible serious spinal pathology); advice given to clients; sickness certification; requests for $\mathrm{x}$-rays; manipulation; acupuncture; following clients up; demographic details; plus a visual analogue scale to ascertain how satisfied G.Ps felt with their current approach to the management of acute low back pain.

As in the pilot study, the questionnaire was printed on 'chamois' coloured paper and coded to enable follow-up reminders to be sent, (but the code was known only to the researcher). This final questionnaire was then sent to all G.Ps in Southampton and the New Forest $(\mathrm{n}=236)$, with an introductory letter about the study. The participants were identified from one health district in a F.H.S.A. practitioner listing of the former south and west region, and included all G.P. practices in the area (except two, deemed atypical, which were linked to the University of Southampton). A stamped addressed envelope was included for their reply. As in the pilot study, non-responders were followed-up initially by letter at two weeks. However, if there was still no reply, a further follow-up letter was sent at one month.

\subsection{Results of the final G.P. questionnaire}

The response rate was 59/97 (61\%) in the New Forest, 107/139 (77\%) in Southampton, giving a total of $166 / 236(70 \%)$. There were no obvious differences between responders and non-responders in the number of practice partners (median $=5$ for both), being in a training practice ( $48 \%$ and $47 \%$ respectively), or geographical location ${ }^{(22)}$. The urban or rural nature of each practice was assessed by using O.P.C.S. Classification ('wholly urban' practices $72 \%$ and $64 \%$ respectively $)^{(22)}$. 
A summary of the descriptive data can be found in appendix 3.4. The only results that will be addressed in this chapter are those relating to possible empowerment issues and demographic details of the G.P. population.

\subsubsection{Demographic details about the sample}

The results from asking participating G.Ps the duration they had been practising as a G.P., together with their completion of any post-graduate courses on the management of acute low back pain, are summarized in table 3.7 .

\section{Table 3.7: Demographic characteristics of the G.P. population}

Results summarizing the demographic characteristics of the G.Ps in Southampton and the New Forest who responded to the final questionnaire $(n=166)$ :

\begin{tabular}{|c|c|c|c|}
\hline $\begin{array}{l}\text { How many years have you been } \\
\text { practising as a G.P? }\end{array}$ & $\begin{array}{l}\text { range } \\
\text { mean } \\
\text { median }\end{array}$ & $\begin{array}{l}=1-37 \text { years } \\
=14.3 \text { years } \\
=14 \text { years }\end{array}$ & \\
\hline $\begin{array}{l}\text { Have you completed any post-graduate } \\
\text { courses on the management of acute low } \\
\text { back pain? }\end{array}$ & $\begin{array}{c}\text { yes }=53 \\
(32 \%)\end{array}$ & $\begin{array}{l}\text { duration range } \\
\text { mean duration } \\
\text { median duration }\end{array}$ & $\begin{array}{l}=1-16^{\dagger} \text { days } \\
=4.4 \text { days } \\
=5 \text { days }\end{array}$ \\
\hline
\end{tabular}

tOne G.P. cited their postgraduate course duration as 13 months, but it was unclear whether this was fulltime or not, and so to avoid skewing the mean, this was excluded from the analysis.

As in the pilot study, to investigate whether practitioners who are more experienced in general practice are more likely to have completed any relevant post-graduate training courses, a chi-squared test can be done if the duration of practising as a G.P. is analysed as a binary variable (i.e. $<14.3$ years [the mean] or $\geq 14.3$ years). The result $\left(\chi^{2}=4.221\right.$ with $\mathrm{df}=1$ ) is significant at the 0.05 level, thus suggesting that G.Ps who have been practising longer are more likely to have undertaken post-graduate training in the management of acute low back pain than their more recently qualified colleagues. This finding differs from the smaller, pilot study.

In addition to experience and training, another important consideration is the time a G.P. is able to spend with each client. It may be perceived that G.Ps with longer appointment times in their clinics may offer more thorough assessments and advice to their clients, 
and so it was necessary to consider surgery intervals. The results are summarized in table 3.8 .

\section{Table 3.8: Surgery interval results from the final G.P. questionnaire}

Results from the final questionnaire asking G.Ps to identify their consultation interval in routine surgeries $(n=166)$.

\begin{tabular}{|l|ll|}
\hline What is your consultation interval in & range $=5-15$ minutes \\
routine surgeries? & mean $=9.1$ minutes \\
& median $=10$ minutes \\
& mode $=10$ minutes $(\mathrm{n}=102)$ \\
\hline
\end{tabular}

Since $61 \%$ of G.Ps $(n=102)$, had identical surgery intervals of ten minutes, the considerable variations in assessment and advice given were not due to the length of appointments alone. This is important when considering what advice is offered to clients and is discussed further in section 3.6.

\subsubsection{How satisfied were G.Ps?}

Generally doctors reported themselves as being not very satisfied with their current approach to managing back pain, with one third of them $(n=55 / 165)$ rating their satisfaction with their back pain management as 4 out of 10 or less on a visual analogue scale (where $0=$ totally unsatisfied and $10=$ totally satisfied). The median satisfaction score $=5.1^{(22)}$, the range was $1.0-8.5$ and the mean $=5.2$.

Possible reasons for this might include a perceived lack of training, conflicting reports of research findings, a rapid natural history of resolution, no known cure, the high prevalence of back pain among the general population, high recurrence rates, and previous experience of managing clients with acute low back pain.

\subsubsection{How do G.Ps assess?}

As suggested in section 3.2 the G.P. has great potential to empower a client through aspects of their clinical examination. For example, clinical tests could be undertaken and feedback given to the client to indicate that no serious spinal pathology is likely, and thus further discussion between doctor and client could then include possible self-help management strategies, if this seemed appropriate. 
Assessment of clients by G.Ps comprises three essential elements, firstly eliminating any possible serious spinal pathology (danger signs), and then taking a verbal history, followed by a physical examination. Regarding the danger signs question specifically, the results were as shown in table 3.9 .

\section{Table 3.9: Danger signs results from the final G.P. questionnaire}

Results from the final questionnaire asking G.Ps to identify whether they considered the following 8 items to be danger signs for a patient with acute low back pain. The results show the numbers and percentages who responded 'yes' to each item. When the total response is less than 166, this signifies some G.Ps omitted this item.

\begin{tabular}{||l|l|c|c||}
\hline \multirow{4}{*}{} & & $\begin{array}{c}\text { Yes } \\
(\mathbf{n})\end{array}$ & $\begin{array}{c}\text { Yes } \\
(\%)\end{array}$ \\
\hline 'Probable danger signs' & Extensor plantar response & $83 / 150$ & 55 \\
\cline { 2 - 4 } & Neurological signs at multiple levels & $135 / 159$ & 85 \\
\cline { 2 - 4 } & Saddle anaesthesia & $147 / 157$ & 94 \\
\hline 'Possible danger signs' & [Constant] night pain & $47 / 150$ & 31 \\
\cline { 2 - 4 } & Bilateral leg signs & $84 / 157$ & 54 \\
\hline 'Probably not danger signs' & Severe local back pain & $12 / 156$ & 8 \\
\cline { 2 - 4 } & Loss of reflex at one level & $16 / 152$ & 11 \\
\cline { 2 - 4 } & Unilateral sciatic symptoms below the knee. & $4 / 152$ & 3 \\
\hline
\end{tabular}

From these results it can be seen that the G.Ps in our survey considered 'saddle anaesthesia' to be the danger sign of most concern and 'unilateral sciatic symptoms below the knee' to be of least concern.

It is encouraging that our three 'probable danger signs' coincided as the three signs G.Ps considered most likely to be worthy of seeking immediate hospital advice for a client with acute low back pain. However, the proportion of doctors who did not consider these as danger signs was surprisingly high.

One possible explanation for this could be that G.Ps feel confident in their management of patients with acute low back pain, although this seems unlikely from their dissatisfaction scores recorded using the visual analogue scales, (although measuring 
satisfaction is not necessarily an indication of confidence). Other possible explanations for these results could be that G.Ps underestimate the significance of these clinical signs, or that they are aware of the controversies surrounding the literature and favour not seeking immediate hospital advice. Since the introduction of clinical guidelines in the U.K. ${ }^{(1,49)}$, it would be interesting to reassess this issue to see whether there is now greater awareness of danger signs. Further work is planned following this thesis.

When comparing our hierarchy of danger signs with the C.S.A.G. report ${ }^{(1)}$, the latter does not specifically mention extensor plantar response or night pain, both of which featured strongly with the Southampton practitioners, (although constant, progressive and nonmechanical pain was identified as a symptom which suggests possible serious spinal patholog $\left.y^{(1)}\right)$. Evidence from the literature suggests that it would be prudent to add these two signs and symptoms to the list outlined in the C.S.A.G. report.

Having considered the elimination of any possible serious spinal pathology, the results of the routine physical tests that G.Ps claimed to perform in a first examination of a client with acute low back pain are shown in table 3.10.

\section{Table 3.10: Physical examination results from the G.P. questionnaire} Results from asking G.Ps to identify which tests they routinely perform in a first examination of a patient with acute low back pain. The results show the numbers and percentages who responded 'yes' to each item. When the total response is less than 166 , this signifies some G.Ps omitted this item.

\begin{tabular}{|l|c|c||}
\hline & $\begin{array}{l}\text { Yes } \\
\text { (n) }\end{array}$ & $\begin{array}{l}\text { Yes } \\
(\%)\end{array}$ \\
\hline Straight leg raising & $147 / 163$ & 90 \\
\hline Reflexes & $117 / 161$ & 73 \\
\hline Muscle weakness & $64 / 156$ & 41 \\
\hline Sensation & $70 / 158$ & 44 \\
\hline Palpation of the spine & $156 / 163$ & 96 \\
\hline
\end{tabular}

From these results, palpation of the spine is routinely performed most commonly by G.Ps whereas muscle weakness is tested least often. This is interesting because clinical guidelines recommend a diagnostic triage: eliminating serious spinal pathology, and 
then distinguishing a nerve root problem from 'simple backache'(1). To clinically diagnose radicular symptoms due to nerve root compression, Bogduk ${ }^{(54)}$ states, the 'radicular pain must be accompanied by other features of nerve compression: numbness, weakness, or paraesthesia'. This can be determined by testing sensation and muscle weakness, the two least-commonly performed tests by G.Ps in clinical practice.

It would be interesting to establish why practitioners do not usually attempt to clinically diagnose radicular pain. Whilst there are little or no published reports as to why this is so, possible reasons might include:

- not considering this an important differentiation, despite the recommendations of clinical guidelines

- being unaware of this aspect of clinical diagnosis

- perhaps they think that giving the pathology such a label will not change their management of the client

- possibly being conscious of not wanting to promote illness behaviour (especially if this further assessment will not change their management strategy).

\subsection{How do G.Ps empower their clients?}

Apart from potentially empowering clients through their assessment and elimination of serious spinal pathology, G.Ps have considerable potential to influence whether (or not) clients feel empowered, by any advice that they may offer. The content of the advice is important, to ensure that it is relevant to the client and their lifestyle. It is also worth considering how this advice is delivered. If it is 'prescribed' then this will do little to empower the client. By contrast, if the advice is negotiated, or alternatives are offered, then the client is empowered to make decisions and take a more active role in their rehabilitation.

The first stage in investigating whether G.Ps empower their clients by the advice they offer is identifying the topics and the frequency of advice given. (The manner of delivery was not specifically addressed in this thesis.) In other work, Evans and Richards ${ }^{(43)}$ stated that $37 \%$ of patients consulting their G.P. for low back pain received 
advice, but they have not attempted to categorize the advice given. In our study G.Ps were asked to identify any advice that they claimed to offer in seven categories. A summary of the results can be seen in table 3.11 .

\section{Table 3.11: Specific advice given by G.Ps}

Results from asking 166 G.Ps to specify any advice they might give to a patient with acute low back pain. The results show the numbers and percentages of G.Ps who responded 'yes' to each item. When the total response is less than 166 , this signifies some G.Ps omitted this item. The ordering of these items mimics the wording of the question (i.e. random) to minimize cueing of the G.P. responses.

\begin{tabular}{||l|c|c||}
\hline & $\begin{array}{c}\text { Yes } \\
(\mathbf{n})\end{array}$ & $\begin{array}{c}\text { Yes } \\
(\%)\end{array}$ \\
\hline Sleeping / mattress & $148 / 163$ & 91 \\
\hline Driving & $108 / 164$ & 66 \\
\hline Specific back exercises & $95 / 163$ & 58 \\
\hline General fitness & $105 / 158$ & 66 \\
\hline Sitting & $134 / 162$ & 83 \\
\hline Lifting & $159 / 164$ & 97 \\
\hline Gardening & $82 / 158$ & 52 \\
\hline
\end{tabular}

Shortage of time has been cited as a major factor in general practitioners' failure to realise their potential in health promotion ${ }^{(8)}$. In their study of 10 general practices with appointment lengths of $7 \frac{1}{2}$ minutes or less, Wilson et al. found that increasing the consultation times to ten minutes caused an increase in some aspects of health promotion $^{(8)}$. However, this work was in all aspects of primary care and no such study has ever been reported for low back pain. In our study, only 58 G.Ps (35\%) had less than 10-minute appointment times, and using Wilson's arguments this should have been sufficient time to include at least some aspects of health promotion ${ }^{(8)}$. It was encouraging to see that only one G.P. claimed to offer no advice in any of the seven categories identified. The next stage was to explore what specific details G.Ps had recorded in the questionnaire i.e. what advice did they actually give in clinical practise? 
It has been shown that appropriate information and advice can reduce anxiety and improve clients' satisfaction with care ${ }^{(49)}$. This is important, since anxiety acts as a barrier to communication ${ }^{(55)}$, which may therefore prevent clients understanding or remembering what has been said. Buchanan reports that between $7 \%$ and $53 \%$ of patients claim not to have understood what their doctors have told them ${ }^{(55)}$. Even assuming clients have understood what their G.P. told them, giving advice is not just an easy option for the health professional, as one G.P. in our survey said:

'I feel that due to patient expectations and demands, the patient expects the doctor or sometimes the physiotherapist to cure their back problem. Advice re: exercise and general health are not easily accepted as it means the patient him/herself needs to make an effort. Also with general pressures on jobs, patients cannot always do the right things for their backs or they risk losing the job'.

The C.S.A.G. report advocates clients should be given '... simple, practical advice on how to avoid excessive loads on the back while performing everyday activities. Advice should be tailored to the patient's individual life and work ${ }^{(1)}$. The A.H.C.P.R. guidelines $^{(47)}$ ‘... define a paradigm shift away from focusing care exclusively on the pain and toward helping patients improve activity tolerance'. However, it must be remembered that at the time of undertaking the G.P. survey (in 1993) the C.S.A.G. ${ }^{(1)}$, R.C.G.P. ${ }^{(4)}$, A.C.C. ${ }^{(51)}$ and A.H.C.P.R. ${ }^{(47)}$ clinical guidelines were not yet published. Incidentally, even though they are now published and in circulation, the majority of these guidelines offer little or no specific advice about sleeping / mattresses, driving, sitting, lifting or gardening.

Much advice given by G.Ps is presumably based on common sense, since the evidencebase for these issues is surprisingly small. This apparent paucity of evidence can undermine a doctor's confidence e.g. one G.P. in our study commented they were 'Not happy with how to advise patients'. The Back Book ${ }^{(56)}$, which the authors claim is 'based on the latest medical evidence', does contain some advice, but much of it appears anecdotal as opposed to research-based, and again it was not available at the time of our survey. The results of our survey are shown in tables $3.12-3.18$. 


\subsubsection{Advice about sleeping / mattresses}

Regarding sleeping / mattresses, a total of 130 G.Ps claimed to give specific advice, while a further 18 said they gave advice, but failed to include any details. The responses given have been analysed into five categories, and summarized in table 3.12 .

\section{Table 3.12: Specific advice given by G.Ps about sleeping / mattresses}

Results from asking 166 G.Ps to specify any advice they might give to a patient with acute low back pain about sleeping / mattresses.

\begin{tabular}{||l|c|}
\hline Advice re: sleeping / mattresses & Number of G.Ps giving advice \\
\hline Firmness of mattress / bed & 92 \\
\hline Positioning & 21 \\
\hline Aspects of the bed e.g. height & 8 \\
\hline Use of pillows / neck support & 7 \\
\hline Other tips e.g. lumbar roll & 2 \\
\hline
\end{tabular}

Overwhelmingly, the issue of firmness of the mattress or bed was most commonly addressed ( $71 \%$ of responses), presumably with advice similar to the Back Book ${ }^{(56)}$, which states '... some people prefer a firm mattress - or try boards beneath your mattress', but if the client lives alone, getting the latter into position may not be feasible with back pain.

Perhaps the most useful advice offered is how to test the firmness of the bed, since

'What's firm to someone of 8 stones won't be much good to another of 16 stones' ${ }^{(57)}$. To test the firmness of the bed, clients are advised to lie supine and slip the flat of their hand into the lumbar lordosis. The hand should slip in quite easily, but the mattress should be '... pushing up to fill the gap'. If the gap remains, the bed is considered too hard. If on the other hand, the person sinks in '... too far and finds it awkward to turn', then the mattress is deemed too soft ${ }^{(57)}$. Unfortunately this information appears to be anecdotal as opposed to research-based, nevertheless it is a start, offering clients practical advice on helping them to decide what appears 'right' for them. 


\subsubsection{Advice about driving}

Eighty-nine practitioners claimed to give advice about driving whilst 19 more were nonspecific. The majority of comments focused on minimizing this activity where possible, but if it has to be undertaken, then how to modify the drivers' position. Few comments $(n=9)$ suggested adjustments to the car seat, and these were placed in a separate category to modifying the drivers' position as it was unclear whether these were rapidly attainable adjustments, such as a lumbar roll, or moving the seat forward, or longer-term solutions such as changing the car seat (and therefore the car).

\section{Table 3.13: Specific advice given by G.Ps about driving}

Results from asking 166 G.Ps to specify any advice they might give to a patient with acute low back pain about driving.

\begin{tabular}{|l|c|}
\hline Advice re: driving & Number of G.Ps giving advice \\
\hline Modify your position & 38 \\
\hline Warnings e.g. avoid / reduce & 38 \\
\hline Adjustments to the car seat & 9 \\
\hline Other $\boldsymbol{e}$. g. regular extension exercises & 4 \\
\hline
\end{tabular}

The Back Book recommends '... adjust your seat from time to time' and '... try a folded towel in the small of your back ${ }^{\text {(56) }}$. More comprehensive details are included in the National Back Pain Association leaflet 'Better backs for drivers' ${ }^{\text {'(58), }}$, although again, the evidence-base for the content of this leaflet is unclear.

\subsubsection{Advice about specific back exercises}

There was considerable debate with advising specific back exercises as this topic is controversial. In our survey, 76 practitioners gave details of their advice, whilst 19 more claimed to give advice, but omitted any details.

Because 21 doctors delegated responsibility for selecting exercises to physiotherapists or to clients themselves (via books), and 11 gave a general answer saying it depended on the stage and diagnosis, only 36 G.Ps recommended specific exercises. Exercises recommended included either flexion, extension, lateral flexion, abdominal exercises or 
hanging, (since it was unclear whether 'strengthening', 'mobility' and 'fitness' were specific or general).

\section{Table 3.14: Specific advice given by G.Ps about specific back exercises}

Results from asking 166 G.Ps to specify any advice they might give to a patient with acute low back pain about specific back exercises.

\begin{tabular}{||l|c|}
\hline Advice re: specific back exercises & Number of G.Ps giving advice \\
\hline Extension & 25 \\
\hline Refer $e . g$. to physiotherapy, books etc. & 21 \\
\hline Depends on stage and diagnosis & 11 \\
\hline Other $e . g$. abdominal exercises, hanging & 5 \\
\hline Flexion & 5 \\
\hline Mobility & 3 \\
\hline Fitness & 3 \\
\hline Strengthening & 2 \\
\hline Lateral flexion & 1 \\
\hline
\end{tabular}

There is still much controversy surrounding specific back exercises, as Deyo says 'No single exercise is best' ${ }^{(2)}$. The Back Book addresses this by saying 'Different exercises suit different people. Find out what suits your back best ${ }^{\text {(56) }}$, which is of limited use to a confused client.

The C.S.A.G. report advocates active rehabilitation programmes in preference to specific back exercises, suggesting there is little scientific evidence to support the value of any specific form of back exercise ${ }^{(1)}$, although the R.C.G.P. guidelines argue that "McKenzie exercises may produce some short-term symptomatic improvement in acute low back pain ${ }^{\text {(49) }}$. Since the publication of both of these guidelines, there is a sound research base developing for advocating exercises designed to improve the function of the multifidus ${ }^{(59)}$ muscle and further studies are awaited on the specific effects of training the transversus abdominis and oblique muscles in clients with back pain.

Frost et al. reported in a single blind randomized controlled trial of clients with chronic low back pain, that simply advising clients to become more active is not effective, and additional support is necessary to change pain, disability, belief in self-efficacy and 
functional capacity ${ }^{(60)}$. Perhaps these findings need to be considered when advising clients with acute low back pain, and primary care clinicians may need to be mindful of means of offering 'additional support', such as a follow-up telephone call, or face-to-face appointment.

Further work is clearly needed, perhaps using imaging techniques, such as real-time ultrasound imaging to measure muscle cross-sectional area ${ }^{(59)}$, to identify any positive or negative effects of specific back exercises on acute low back pain and help resolve this on-going issue.

\subsubsection{Advice about general fitness}

Regarding general fitness, 74 of our G.Ps identified specific advice whilst 31 were nonspecific. The results are shown in table 3.15.

The evidence-base for general fitness is less controversial than for specific back exercises, with all the clinical guidelines advocating staying active $\mathrm{e}^{(1,47,49,51)}$, together with the Back Book ${ }^{(56)}$, and other authors ${ }^{(43)}$. The Back Book goes further to specifically recommend walking, using an exercise bike or swimming ${ }^{(56)}$.

\section{Table 3.15: Specific advice given by G.Ps about general fitness}

Results from asking 166 G.Ps to specify any advice they might give to a patient with acute low back pain about general fitness.

\begin{tabular}{|l|c|}
\hline Advice re: general fitness & Number of G.Ps giving advice \\
\hline Activities e.g. swimming, yoga, walking & 37 \\
\hline Diet and weight control & 19 \\
\hline Encourage regular exercise & 9 \\
\hline Progression of exercise & 5 \\
\hline Other e.g. back care etc. & 3 \\
\hline Avoid jarring or lifting & 1 \\
\hline
\end{tabular}

\subsubsection{Advice about sitting}

Regarding sitting, 92 practitioners claimed to give specific advice whilst 42 more omitted to include any details. 


\section{Table 3.16: Specific advice given by G.Ps about sitting}

Results from asking 166 G.Ps to specify any advice they might give to a patient with acute low back pain about sitting.

\begin{tabular}{|l|c|}
\hline Advice re: sitting & Number of G.Ps giving advice \\
\hline Positioning & 67 \\
\hline Warnings e.g. duration, not in acute stage & 14 \\
\hline Chair details & 8 \\
\hline Applied to occupation & 3 \\
\hline
\end{tabular}

Despite the lack of evidence in clinical guidelines, Nachemson's work on lumbar disc pressure measurements would suggest that sitting increases the discal pressure when compared to standing or lying ${ }^{(61)}$, and it is therefore likely to increase pain if sitting takes place in the acute stage of low back pain. The Back Book advocates using an upright chair, trying a folded towel to support the lumbar lordosis and getting up to stretch every $20-30$ minutes $^{(56)}$.

\subsubsection{Advice about lifting}

Of the seven categories identified, advice was given most frequently about lifting, with 119 practitioners specifying the advice they offer and a further 40 reportedly giving advice, but omitting any details.

\section{Table 3.17: Specific advice given by G.Ps about lifting}

$\overline{\text { Results from asking } 166 \text { G.Ps to specify any advice they might give to a patient with }}$ acute low back pain about lifting.

\begin{tabular}{||l|c|}
\hline Advice re: lifting & Number of G.Ps giving advice \\
\hline Technique & 80 \\
\hline Warnings e.g. avoid twisting, strain etc. & 27 \\
\hline Refer e.g. to physiotherapy, books etc. & 6 \\
\hline Other e.g. get help & 3 \\
\hline Mechanical principles / physics & 2 \\
\hline Application to occupation & 1 \\
\hline
\end{tabular}


Information about technique was overwhelmingly the most frequently addressed aspect $(n=80)$. Again evidence for these issues can be drawn from Nachemson's work, which estimates the relative change in pressure (or load) in the third lumbar disc in various positions and lifting activities ${ }^{(61)}$.

\subsubsection{Advice about gardening}

This category was the least frequently addressed one of the seven, perhaps because it is not so relevant for all clients, perhaps because G.Ps feel less confident to advise, or perhaps because of the paucity of research evidence?

\section{Table 3.18: Specific advice given by G.Ps about gardening}

Results from asking 166 G.Ps to specify any advice they might give to a patient with acute low back pain about gardening.

\begin{tabular}{||l|c|}
\hline Advice re: gardening & Number of G.Ps giving advice \\
\hline Warnings e.g. avoid / reduce no lifting etc. & 38 \\
\hline Technique & 12 \\
\hline Other e.g. pacing self, duration etc. & 10 \\
\hline Equipment e.g. small spade, long handles & 6 \\
\hline Refer e.g. to leaflets / books & 2 \\
\hline
\end{tabular}

Probably the most comprehensive, readily available advice is the National Back Pain Association leaflet entitled 'Better backs for gardeners' ${ }^{9}$, although as before, the evidence-base for much of the content is unclear. The content was apparently determined by health professionals alone ${ }^{\dagger}$.

\subsubsection{The use of leaflets}

Only $35 \%(n=58)$ responded that they gave any leaflets on back to patients. Doctors were asked to send back a copy of any leaflets they gave out to clients with the questionnaire, and several different leaflets were returned. The majority of these were produced by pharmaceutical companies and none of the advice they contained appeared

\footnotetext{
† Source: BaceCare, formerly the National Back Pain Association.
} 
to be supported by any published evidence. Indeed some advice was misleading or even incorrect. For example appendix 3.5 shows a leaflet, which is claimed to be a '... recommended regime of conservative care' ${ }^{\text {(63) }}$. The leaflet was produced 'with the aid of a panel of back specialists to ensure the information is accurate and enables patients to gain relief as quickly and as completely as possible ${ }^{(63)}$. The leaflet, which advises up to six weeks bed rest, goes against all published research findings which underpin the clinical guidelines ${ }^{(1,47,49,51)}$. Such information is not only inaccurate, it is potentially detrimental to clients.

\subsubsection{What treatment modalities do G.Ps offer?}

Acupuncture: In our study, when G.Ps were asked to identify the percentage of patients with acute low back pain that they (themselves) treated with acupuncture, $9 / 157$ reported using this modality. Estimates of the percentage of patients that they treated varied from $5-50 \%$, with a median of $15 \%$.

Manipulation: When compared to acupuncture, a greater number of Southampton and New Forest G.Ps reported practising manipulation themselves $30 / 163$ (18\%), and the estimates of the percentage of patients with acute low back pain that they claimed to treat using this modality ranged from $2-95 \%$, with the median $=20 \%$. This was a higher proportion than expected.

The ability to treat clients can affect the doctors' self-reported satisfaction score of their management of clients with acute low back pain. For example one G.P. described themselves as 'inadequate as I have no physical skills. I would like to use manipulation techniques'. Further evidence can be seen as the mean satisfaction score measured on a $10 \mathrm{~cm}$ V.A.S. (see 3.5.2) of doctors who practised acupuncture $=6.4$ compared with those who did not practise acupuncture, whose mean score $=5.1$. Likewise satisfaction scores of practitioners who were performing manipulation were more satisfied with their management of clients with acute low back pain, since they had a mean satisfaction score of 6.4 compared to those who did not use these techniques, whose mean score was 4.9.

Regarding acupuncture, Evans and Richards ${ }^{(43)}$ state that its use '... tends to be confined to chronic low back pain', but the authors offer no supporting evidence for this statement. However our results show that $6 \%$ of practitioners used acupuncture 
specifically to treat clients with acute low back pain, suggesting it is perhaps used more widely than Evans and Richards perceived.

One possible factor that has restricted the evidence base for acupuncture is the methodological difficulty of undertaking a research trial with an appropriate control group, and the obvious problems of 'blinding' clinicians and participants. This has resulted in reports stating that as yet '... there is no evidence on the efficacy of acupuncture for acute low back problems ${ }^{(49)}$. However, not all clinical guidelines share this view since the C.S.A.G. report ${ }^{(1)}$ identifies acupuncture as an option for symptomatic relief, one of a number of therapeutic options, which '... should not be regarded as treatments in themselves', but which can be valuable in facilitating active exercise and rehabilitation.

It is not surprising that manipulation is practised more commonly by practitioners than acupuncture, since it has a stronger evidence-base ${ }^{(1,49)}$, and greater opportunities for postgraduate training. However, the use of acupuncture by health professionals (such as G.Ps and physiotherapists) is becoming more widespread, indicating that attitudes towards acupuncture are changing ${ }^{(64)}$.

From the results of this survey, clearly the ability to actually treat clients is satisfying to doctors, but this cannot be assumed to necessarily empower clients. Some clients may benefit from acupuncture or manipulation and be empowered to actively rehabilitate (thereby demonstrating an internal locus of control), whereas others may become dependent upon their G.P. (thereby demonstrating an external locus of control implicating powerful [medical] others). This issue is complex since it involves control issues for both the G.P. and the client, as well as issues of communication in their therapeutic relationship.

\subsubsection{Why do G.Ps refer clients for x-rays?}

The decision whether a G.P. should refer a client for an x-ray is likely to be multifactorial, involving both medical and social agendas ${ }^{(65)}$. In the final G.P. questionnaire, participants were asked to identify the criteria they use when deciding whether $\mathrm{x}$-rays are appropriate for a client with acute low back pain. The purpose was to identify reasons for x-ray referral as perceived by G.Ps. They were asked to identify the criteria in two 
categories - 'medical' reasons and 'social' reasons, following the results of the pilot study (3.4.3).

A total of 319 'medical' reasons were identified (mean 1.9 per G.P.), which were grouped into eight categories in the simplest form of analysis. These categories were not pre-determined, they emerged from the data. (One omission at this stage was getting the data independently analysed by a second, impartial researcher, as this could have ensured a degree of reliability with this process. This was not done however, and this oversight was rectified in further analyses with similar methods, such as in section 7.5.2.)

The results are summarized in table 3.19. A total of 127 'social' reasons were identified (mean 0.8 per G.P.) although one response was unreadable and two others consisted of just a question mark. The 124 usable responses are summarized in table 3.20.

\section{Table 3.19 'Medical' reasons for requesting an x-ray}

Results from asking G.Ps to specify any 'medical' criteria they use in deciding whether $\mathrm{x}$-rays are appropriate for a patient with acute low back pain. A total of 319 comments were identified from a total of 166 G.Ps:

\begin{tabular}{|l|c|}
\hline 'Medical' reasons & $\begin{array}{c}\text { Number of } \\
\text { responses }\end{array}$ \\
\hline Duration e.g. persistence / recurrence & 89 \\
\hline Signs and symptoms & 71 \\
\hline Screening e.g. exclude malignancy, ankylosing spondylitis, sacroilitis etc. & 46 \\
\hline Patient characteristics e.g. age, gender, family history & 35 \\
\hline Pathology e.g. crush fracture, degeneration, spondylolisthesis & 29 \\
\hline Other e.g. pre-referral, if it will alter management etc. & 23 \\
\hline History e.g. trauma, onset & 17 \\
\hline Rarely / none & 9 \\
\hline
\end{tabular}

From the tables, some G.Ps appeared unclear about the 'medical' and 'social' categories e.g. citing 'pathology' in both, although the majority were able to differentiate. From these results it is clear that 'social' reasons form a significant proportion of the reasons why doctors request $\mathrm{x}$-rays $(28 \%)$. Such reasons are not addressed in clinical guidelines which confirms a mismatch between these guidelines and actual clinical practise. When 
a G.P. is 'at the coal-face' with a persistent client, it is understandable that they may feel pressured. One G.P. in our study wrote:

'Patients believe an $x$-ray will somehow help to cure them - difficult to convince them otherwise'.

\section{Table 3.20 'Social' reasons for requesting an $x$-ray}

Results from asking G.Ps to specify any 'social' criteria they use in deciding whether $\mathrm{x}$-rays are appropriate for a patient with acute low back pain. A total of 127 comments were identified from a total of 166 G.Ps ( 3 were unusable):

\begin{tabular}{|l|c|}
\hline 'Social' reasons & $\begin{array}{c}\text { Number of } \\
\text { responses }\end{array}$ \\
\hline Patient pressure / dissatisfaction & 29 \\
\hline Rarely / none & 24 \\
\hline Work related & 19 \\
\hline Other e.g. level of inconvenience, travelling factors etc. & 12 \\
\hline Psychological e.g. reassure patient & 11 \\
\hline Duration $i . e$. no improvement & 9 \\
\hline Patient characteristics e.g. age, if frustrated, lifestyle factors etc. & 6 \\
\hline History $e . g$. road traffic accident, trauma & 5 \\
\hline Medico-legal & 4 \\
\hline G.P. aid $e . g$. quick way to end consultation, time-passing manoeuvre etc. & 3 \\
\hline Pathology / diagnosis & 2 \\
\hline
\end{tabular}

The research team was surprised at the honesty and openness of responses such as 'quick way to end consultation' and 'time-passing manoeuvre'. Such responses raise ethical issues regarding the use of invasive and potentially harmful investigations as ways of negotiating an exit from a consultation or to merely pass time. Fortunately such practise is only reported as $2 \%$ of the 'social' reasons and $0.7 \%$ of the total reasons for referring a client for an $\mathrm{x}$-ray, but nevertheless this is likely to be an under-representation (due to G.Ps perhaps being reluctant to admit such reasons, especially with a coded questionnaire as opposed to a completely anonymous one). 
If this trend of using $x$-rays for social reasons is responsible for $0.7 \%$ of the total reasons for referring a client for x-rays, apart from the potential harm to the client of radiation exposure identified in 3.4.4, the cost of such practises annually in the U.K. (identified in $3.4 .4)=0.7 \%$ of $£ 26.7$ million $-£ 60.1$ million, i.e. $£ 186900-£ 420700$, with no obvious benefit to the client. This money could clearly be used more effectively.

\subsubsection{Following clients up after consultation}

When practitioners in our study were asked whether they encourage their clients with back pain alone to take responsibility for making follow-up appointments with them, $27 \%(n=44)$ replied 'always', and the majority, $58 \%(n=97)$, claimed 'usually'. It was only $13 \%(n=21)$ who responded 'sometimes' and $1 \%(n=2)$ who claimed they 'never' encouraged clients to take responsibility for this follow-up. However, this question has an ambiguity, since it may be that G.Ps who 'never' empower the client to take responsibility for this action either dictate when they will see the client again, or they may advise them that they do not need to seek further medical input for this episode. From the limited information available, it is not clear which is the case.

\subsection{Any other comments}

A total of 99 comments were made, which were simply categorized into five groups, by similar means to section 3.6.10.

\section{Table 3.21 Any other comments}

Results from asking G.Ps if they had any other comments. A total of 99 comments were identified and categorized into the following five groups:

\begin{tabular}{|l|c|}
\hline Category of comment & Number \\
\hline Referral patterns and access issues & 42 \\
\hline Clinical management issues & 41 \\
\hline G.P. needs & 6 \\
\hline General comments about acute low back pain & 6 \\
\hline Miscellaneous & 4 \\
\hline
\end{tabular}


Some comments reinforced the importance of sound assessment skills e.g. one G.P. commented:

'I've seen cauda equina complications 6 times, average is 1-2 per lifetime.'

This confirms the need for all G.Ps, without exception, to be able to identify signs and symptoms of possible serious spinal pathology.

Apart from the obvious need for competent assessment skills, another theme identified in the doctors' comments was their ongoing concerns about the management of acute low back pain. As one G.P. said:

'Acute low back pain is a frustrating condition for both Dr and patient'. Another echoed this saying:

'I find it difficult. Rest and analgesics is all I can offer people quickly'.

Giving G.Ps information of what advice they can offer their clients (such as the topics discussed in section 3.6), could be very useful both to improve the morale and ability of practitioners, whilst simultaneously empowering clients. The importance of sound, practical and relevant advice appears to be underestimated, as one G.P. reflected:

'Perhaps the chief educational needs are:- a) identifying which patients are going to benefit by being referred for intervention; b) giving G.Ps the skills to persuade all the other patients to take their analgesics and do their programme of rest and exercise without rushing off to the manipulators / acupuncturists for a "quick" result. Patients find it hard to accept in the 1990s that they may have to have 2 or more weeks getting better slowly and often painfully (as simple analgesics do not relieve all the pain).'

There is a definite need for this lack of advice to be addressed in future editions of clinical guidelines. Perhaps then, giving advice to clients will achieve the recognition it deserves, and it can be considered as a useful management option or adjunct to treatment. Linked to giving advice, G.Ps also need to address clients' expectations and beliefs. If a client understands the likely natural prognosis and natural history of their back pain, the 
pros and cons of any tests or interventions, and the relevant practical advice, this could be the foundation of the empowerment process, which, if the client wishes, should enable them to take an active part in their own health care. It will take time for progress towards this (perhaps idealistic) status.

\subsection{Application}

So what is the clinical significance of this study? Firstly, heightening awareness of sound assessment skills, including being able to identify serious spinal pathology needs to be disseminated to all clinicians who assess clients with acute low back pain in a primary care setting. As Grieve states ${ }^{(38)}$ 'The recognition of neoplastic disease, earlier rather than later, depends more on awareness, vigilance and suspicion rather than a set of rules. One must always be thinking of it, all the time and every time.'

In addition, reviewing the evidence-base for advice offered to a person with acute low back pain could help clarify practical information which could be used to empower clients and give them a perception of having some control over their symptoms. The potential benefits of using advice as a treatment / management modality are perhaps underestimated by both doctors and clients.

Clinical guidelines need to be developed by clinicians for clinicians, and then evaluated, to enable their further development. If a second, G.P. survey were done at some point in the future, the data presented in this chapter (i.e. pre-C.S.A.G. and R.C.G.P. guidelines) could be used for comparison as it could yield useful information on any changes in practise as a result of publication of these guidelines, and hence their impact.

Finally, as part of the 'Back Home' study, we developed a diagnostic guide for optional use by G.Ps participating in the study (see appendix 3.6). The content was developed from the work on danger signs and symptoms described in section 3.4.1. The guide was developed as an aide-mémoire to assist doctors with their physical examination of a client with acute low back pain.

The diagnostic guide was a green, A5 card, designed to fit inside clients' medical notes. The front of the diagnostic guide listed potential danger signs. In addition it listed osteoporosis risk factors which may justify $x$-ray, since the guidelines from the Royal 
College of Radiologists ${ }^{(44,45)}$ suggest that routine, plain $\mathrm{x}$-rays of the lumbar spine are not recommended unless associated with danger signs or bone pathology such as osteoporotic collapse. Listed on the reverse of the card were useful localizing signs (myotomes) and in addition, the guide was also designed as a direct referral card to outpatient physiotherapy if the G.P. deemed this appropriate. The research team identified that it would be difficult to monitor exactly how the diagnostic guide was to be used throughout the study, hence its introduction as an optional guide only.

\subsection{Summary of the chapter}

- There is a consensus that some signs and symptoms exist which if present, may indicate serious spinal pathology. A few danger signs exist which if present, require emergency referral to a spinal surgeon.

- There is a mismatch between clinical guidelines for requesting $\mathrm{x}$-rays and actual practise, particularly regarding 'social' issues.

- The potential benefits of using advice, as a management option for clients with acute low back pain, appear to be under-estimated by clients and doctors. At present the supporting evidence for this advice is limited. 


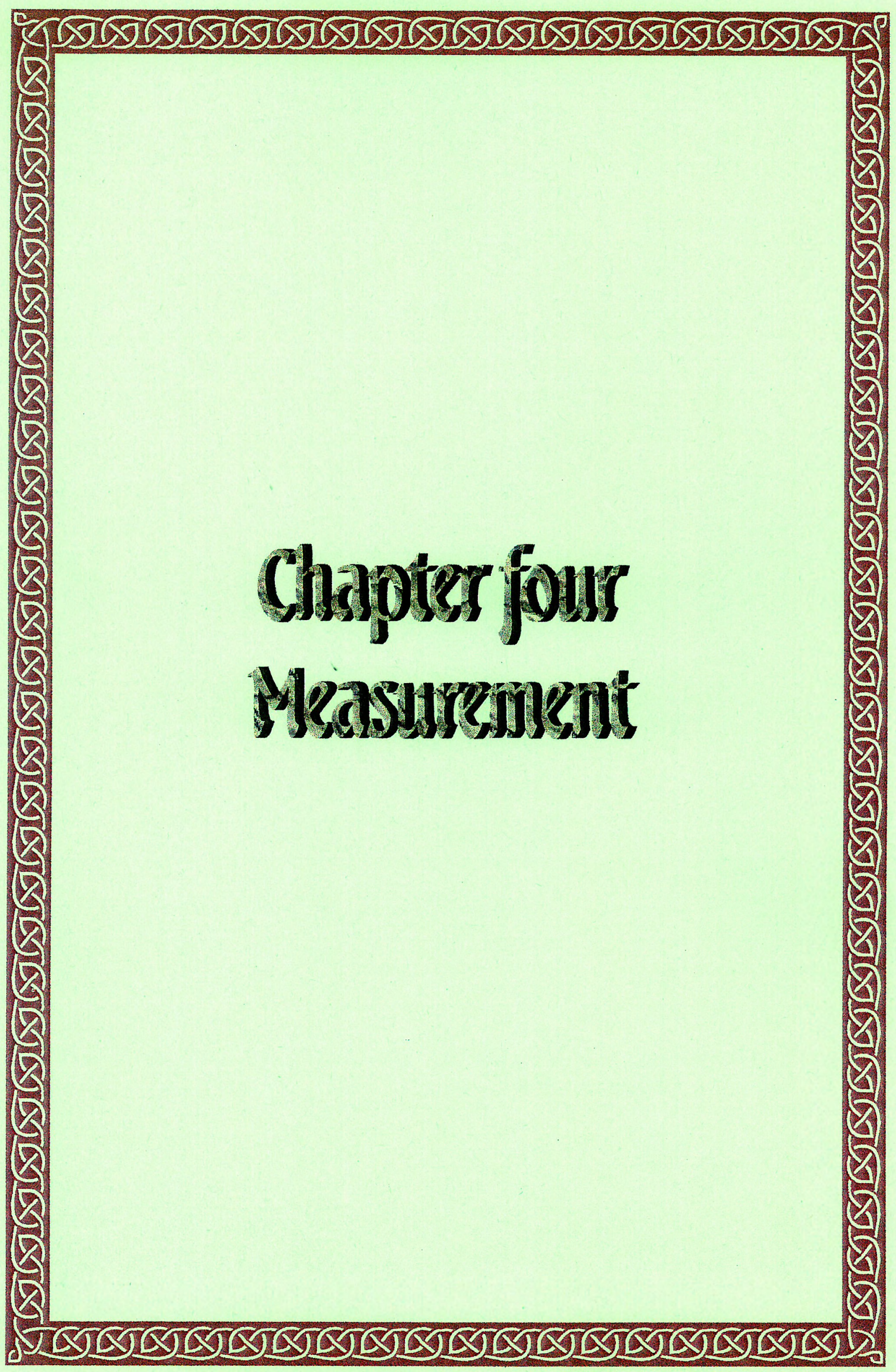




\section{Chapter four: Measurement}

Chapter two outlined the controversy surrounding the definition of 'acute low back pain'. With so little consensus in its definition, it is not surprising that attempts at measuring this symptom are highly contentious.

The selection of appropriate measurement tools was a key issue for the studies in this thesis. However the degree of confusion within the back pain literature hindered progress initially, and so a systematic review was needed to establish the merits of existing measures and inform the final selection of instruments for this work.

This chapter describes the need to measure back pain, our framework (devised to enable the systematic review of existing outcome measures) and the results of four dimensions of interest in this thesis - control, function, pain and anxiety. The strengths and weaknesses of each measure are discussed in detail, and are related to the needs of this research. Having justified the final selection of instruments in this thesis, the chapter concludes with a discussion of the limitations of the framework and review.

\subsection{Operational definitions}

Throughout the health measurement literature, outcome measures are referred to using many different terms such as: instruments; tools; health status indicators; indices; measures; health related quality of life measures; outcome indicators; functional assessments and utility measurements. Such variation may reflect differences in use, for example whether the instrument is used to differentiate between client groups, or to evaluate an intervention.

Since the research in this thesis involves the evaluation of an intervention (a clientinformation leaflet), the term 'outcome measure' is used unless another term is directly quoted, or to avoid undue repetition. For clarity, an 'item' refers to a single question (e.g. how would you rate your present back pain?) and a 'dimension' refers to 'the area of behaviour or experience that we are trying to measure ${ }^{\text {(1) }}$, such as physical function. 


\subsection{Why measure?}

Chapman defines measurement as 'the assignment of numbers to objects or events to represent quantities of attributes according to rules' ${ }^{\text {(2) }}$. Measurement of acute low back pain must therefore be governed by rules (including precise definitions) to ensure that outcome measures are both valid and reliable.

Measurement is of paramount importance. The American College of Physicians state that 'Assessment of the impact of illness on physical, mental, and psychosocial functioning is an essential element of clinical diagnosis, a major determinant of therapeutic choices, a measure of their efficiency, and a guide in the planning of ... care...(3). Such assessment needs outcome measures. Likewise, in physiotherapy, measurements are fundamental to clinical practise. Physiotherapists need a more scientific basis for practise, which is important if we 'are to be recognised as credible health care providers' ${ }^{\text {(4) }}(\mathrm{sic})$.

Specific to back pain, valid, reliable outcome measures will facilitate a better understanding of the natural history of this symptom. Subsequently, it will enable the adoption of more standardized approaches to the evaluation of strategies and interventions in back pain studies, contributing both to clinical practise and research. Such rigorous evaluation can help inform future funding priorities - as Neuberger ${ }^{(5)}$ states, such measures should be 'a way to push towards excellent, effective healthcare, and a way of getting rid of services which are pointless'.

Once appropriate instruments are widely used, another reason for measuring is that population trends can be monitored, for example health care utilization. As well as monitoring how health care is used, it is also important to measure how it is applied. For example, Brooks ${ }^{(6)}$ states that technological changes need to be monitored, although he was using a general context, which is likely to be a modest issue in the conservative management of back pain. However, technological advances will be particularly relevant to spinal surgery.

For all health care providers involved in treating back pain, the challenge of measuring is based on two assumptions: firstly, that acute low back pain can, in fact, be measured (otherwise our study would not have taken place!); and secondly, that individuals are 
able to self-report health issues. Neuberger ${ }^{(5)}$ questions whether it is possible to design measurements that can tell, with sufficient accuracy and reliability, the usefulness of what is being done. The absence of robust measures of back pain, with excellent psychometric properties, is evidence that this issue has not yet been completely resolved. Therefore, the quest for improved outcome measures continues.

Assuming that this is possible to measure acute low back pain, the second assumption is that individuals are able to self-report health issues and cognitive aspects of their pain. Weisenberg defines cognition as embracing the quality of knowing which includes perceiving, recognising, conceiving, judging, sensing, reasoning and imagining, ${ }^{\text {(7) }}$. How an individual processes these qualities is vital for self-report instruments.

\subsection{Are self-report measures appropriate?}

Physiological outcome measures of pain provide information for clinicians, but they are often of limited interest to clients, and frequently correlate poorly with functional capacity and well-being, where clients are most interested and familiar ${ }^{(1)}$. In addition they may require specialized equipment, which may be costly, invasive and impractical (especially in community-based research).

In addition, 'health and illness are social as well as biological facts' ${ }^{\text {(8) }}$. Therefore, to address this issue and enable an individual to record their perceptions about their health status, self-report outcome measures of health i.e. those completed by the client, are important. Some researchers have even suggested that, since health is essentially subjective, the only valid measure to accept is people's own assessment of whether or not, they are healthy ${ }^{(9)}$. Gill and Feinstein ${ }^{(10)}$ argue that "quality of life can be suitably measured only by determining the opinions of patients and by supplementing (or replacing) the instruments developed by “experts" '. Rabbi Neuberger argues strongly for the client viewpoint being included in every health outcome measurement ${ }^{(5)}$. She suggests that client and user groups should be playing a key part in the design of outcome measures so that measurement 'does not simply become a game for health service professionals and health economists, without reference to those who will be directly affected by decisions made ...'. 
Such views are not universal. Hirsch et al. argue that self-reports of back pain are too susceptible to alteration by attitudes and emotions, and suggest that they 'cannot be relied on to guide therapy ${ }^{\text {(11) }}$. It is also considered that self-report measures depend upon client's literacy and ability to concentrate, and are also liable to be influenced by the client's wish to present themselves in a certain light ${ }^{(12)}$. Clearly, self-reporting will be influenced by attitudes and emotions, but perhaps this is an essential component as we are trying to measure people with back pain, and not just back pain per se.

If it is assumed that self-report measures are valuable, then, apart from improving communication with the client, other benefits of measuring health status have been proposed $^{(3)}$ :

- helping clinicians to focus and therefore allocate limited resources;

- decreasing time spent gathering information by asking the client the 'right' questions;

- making good use of otherwise unproductive client waiting time;

- increasing sense of professionalism and job satisfaction in office staff who may be involved with the paperwork;

- increasing client satisfaction, client enjoyment and reassurance at the thoroughness of questioning;

- possibly permitting early detection of illness;

- better differentiation of physical from emotional disability;

- better treatment planning;

- giving useful and encouraging feedback to clients;

- researching clinical effectiveness.

With so many potential benefits, Delitto claims that standardized self-reports should be an integral part of clinical settings in which clients with low back pain are assessed and treated $^{(13)}$. He is keen that the information these measures contain should not be relegated 'to the meaningless drivel often seen in the subjective portion of a problem-oriented medical record (SOAP) ${ }^{,(13)}$. However support for integrating these measures into practise is not universal, for example Deyo states that he is 'not yet convinced that these measures need to be incorporated into everyday practice ${ }^{(14)}$, although he is approaching 
the issue from a medical perspective rather than as a physiotherapist. Golden also postulates that 'Much clinical decisionmaking does not necessarily rely on pin-point accuracy of perceptions of a patient ${ }^{\text {(14) }}$ (sic). Thus, opinion remains divided.

There are difficulties with self-assessments of health, for example what do respondents understand by the term 'health' ${ }^{(9)}$. It may be viewed negatively, as the absence of illness, functionally, as the ability to cope with everyday activities, or positively, as fitness and well-being ${ }^{(9)}$. Having considered this issue, not surprisingly, Blaxter reports that assessments of health have been criticised as 'individual and eccentric'(9). The debate continues.

\subsection{What exactly are we measuring?}

Having purported that low back pain can be measured by self-report measures, to date no single instrument has been developed and no unified measurement approach exists. Consequently many outcome measures are used throughout the health status measurement literature, which Guyatt describes as 'a jungle ... where students of the area must hack through an underbrush of confused terminology and contradictory conceptualisations' $^{\prime(15)}$.

Within this jungle, self-report outcome measures are being increasingly used to look at back pain. Deyo and Tsui-Wu state that 'cultural, social and economic factors may influence the perception of pain and the response to $\mathrm{it}^{{ }^{(16)}}$ : Such inherent factors, may confound measurement results.

When assessing a client with low back pain, just focusing on physical signs and symptoms is clearly insufficient. Bonder argues that due to the holistic nature of function, 'All therapists must assess psychological factors for every client ${ }^{(17)}$. This is in keeping with Waddell's biopsychosocial model ${ }^{(18)}$, which was described in chapter one.

These biopsychosocial factors are not static. Like the natural history of back pain discussed in section 2.7 , they change with time and are therefore different in acute and chronic pain states. In order to measure the changing aspects of acute low back pain, it is therefore inappropriate to select measures that are based on the chronic pain literature as these are designed to measure a relatively more stable phenomenon and reflect a different 
perspective of time. If outcome measures are used interchangeably for acute and chronic low back pain in this way, this implies that acute and chronic pain are identical symptoms, distinguishable solely by duration, which is clearly untrue. Therefore, this practise should cease.

\subsection{Selecting measures}

Although outcome measures provide information, the Task Force on Standards for Measurement in Physical Therapy warn that 'the result may be misinformation if the quality of measurements is not ensured ${ }^{(4)}$. Thus, measuring acute low back pain necessitates reliable, valid and sensitive outcome measures that have been specifically designed for the task. In the 'Back Home' study we needed outcome measures reflecting dimensions of control, function, pain, anxiety, satisfaction, knowledge, attitude, behaviour, together with details of demography and medical management. In six of these dimensions, the search for appropriate outcome measures was limited by one of three factors: i) because the detail required was factual (e.g. information on demography, medical management); ii) only one main measure was deemed appropriate (e.g. knowledge and satisfaction instruments); or iii) no suitable measure could be found (e.g. outcome measures of attitude, behaviour). Therefore the systematic review of outcome measures primarily focused on measures reflecting the four dimensions of control, function, pain and anxiety, where a number of measures exist. It was agreed that wherever possible, existing measurement scales would be used in the 'Back Home' study since designing new measures from scratch is complex, time consuming and expensive. Whilst recognising that no single scale is perfect, it is acknowledged that reviewing a measurement tool is considerably easier than constructing one. Nevertheless, the task still appeared daunting.

Potential outcome measures were identified using current low back pain literature, computerised searches, reviews and reference texts. In order to approach each measure in a systematic way a framework was developed which contained aspects previously identified by Guyatt ${ }^{(19)}$, McDowell ${ }^{(20)}$, Law $^{(21)}$, Bombardier and Tugwell ${ }^{(22)}$, Streiner and Norman ${ }^{(23)}$. Measuring health is an evolving science and the quality of the instruments is improving from early efforts, which Brooks described as often being impractical, used 
vague terminology, had poor reproducibility and showed a lack of comparative weighting and scoring ${ }^{(24)}$. Issues of culture and language needed to be considered alongside the framework and these are discussed further in section 4.13.

\subsection{The framework}

In attempting to simplify the selection of existing outcome measures, a framework was devised, based on what I termed the "3Ps":

- Purpose i.e. ability to measure dimensions of health

- Practical aspects e.g. number of items, style, time needed

- Psychometric properties i.e. reliability, validity, sensitivity to change

Each of these three domains contains a number of items. When selecting an outcome measure, e.g. for clinical practise or a research trial, the balance of each domain in the tripartite framework must be considered. It does not necessarily follow that all three domains are equally important - it depends on the context.

To illustrate this concept, a simple vector diagram may be used. The length of each line represents the relative importance of each domain, thereby weighting its influence. If the purpose, practical aspects and psychometric properties are all considered equally important, the framework may be represented:

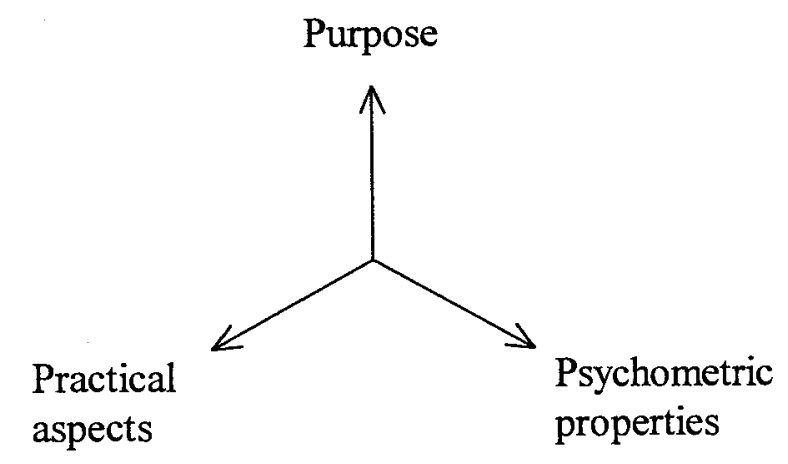

If however, an outcome measure is being selected for use in clinical practise, it may be that the most important consideration is the practical aspects e.g. time factors, administration etc. Therefore practical aspects may influence the selection of outcome measures more than either of the other two domains and the vector may be drawn: 


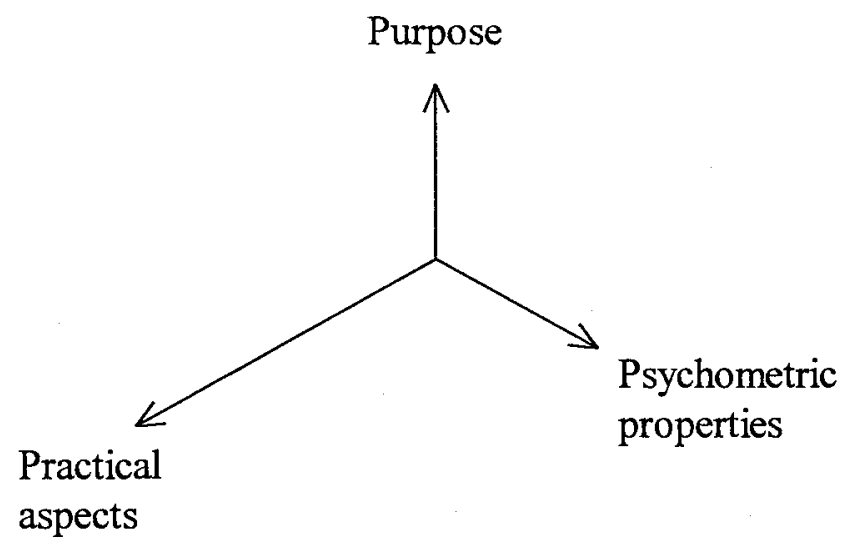

Whereas if the outcome measures were being selected for a research trial, then the psychometric properties may be the most important factors to consider i.e. the reliability, validity and sensitivity to change. These would therefore need to be given a higher priority than perhaps the purpose and practical aspects, thus altering the balance:

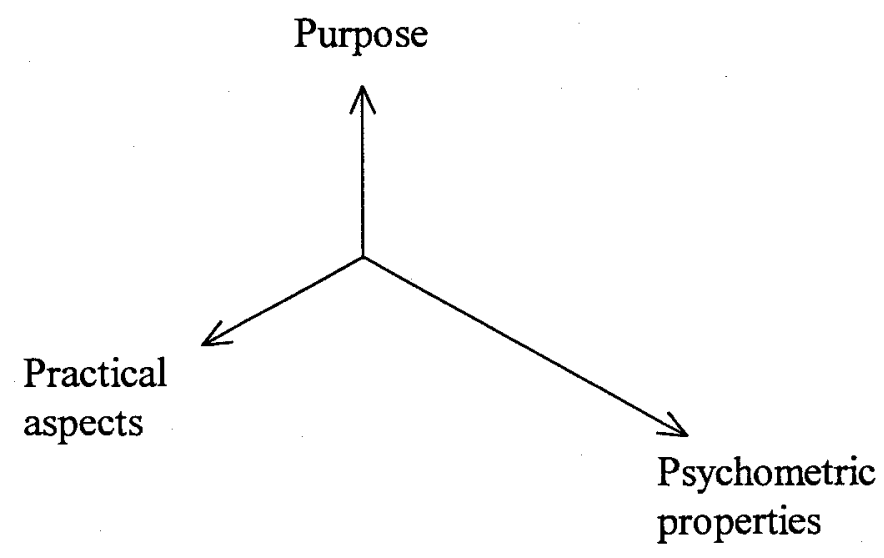

Naturally this simplification has its limitations. However when reviewing any outcome measure, it is essential to consider the weighting of the '3Ps': Are they of equal importance for your particular task?

Having considered this, the framework (now outlined) can be used to review the outcome measure.

a) Title of the outcome measure, year of publication, author(s) and references.

b) Purpose

The purpose of an instrument addresses two agendas. Firstly, what is the underlying intention of the measure? Is it designed to discriminate whether a client has a particular symptom, predict whether a client will develop a particular symptom or evaluate the 
effect of an intervention? Secondly, how is the client affected: is the measure one of impairment, functional limitation, disability or handicap?

In order to address the second aspect, the terminology of the International Classification of Impairments, Disabilities and Handicaps (I.C.I.D.H.) ${ }^{(25)}$ was initially used. This classification has been modified by Nagi to incorporate the additional category of 'functional limitation'(in 26):

\section{Table 4.1: Classification of purpose}

Definitions of impairment, functional limitation, disability and handicap based in the I.C.I.D.H. classification ${ }^{(25)}$, subsequently modified by $\mathrm{Nagi}^{(i n}{ }^{26)}$.

\begin{tabular}{|l|l|}
\hline Impairment & $\begin{array}{l}\text { 'any loss or abnormality of anatomic, physiologic or psychologic } \\
\text { structure or function' }\end{array}$ \\
\hline Functional limitation & $\begin{array}{l}\text { 'inability to perform a task or obligation of usual roles and } \\
\text { typical daily activities as the result of impairment' }\end{array}$ \\
\hline Disability & $\begin{array}{l}\text { The I.C.I.D.H. defines disability in terms of the preceding } \\
\text { definition of functional limitation. } \\
\text { The sociological model developed by Nagi limits the term } \\
\text { disability to 'overall patterns of behaviour in situations of long- } \\
\text { term or continued impairments that result in functional } \\
\text { limitations' }\end{array}$ \\
\hline Handicap & 'the social disadvantage of a disability.' \\
\hline
\end{tabular}

\section{c) Practical aspects}

\section{i) Number of items}

The tables show the number of questions in the original outcome measure, with the length of any major revisions in brackets.

\section{ii) Style}

This includes:

- administration i.e. whether the author(s) consider the measure can be selfadministered 
- type of scale i.e. is it categorical (e.g. a Likert scale), or continuous (e.g. a visual analogue scale)

- Ievel of measurement i.e. are the data:

- nominal (named categories e.g. gender),

- ordinal (ordered categories e.g. educational level),

- interval (when the differences between responses is known and constant e.g. numerical rating scale),

- ratio (when there is a meaningful zero point, so that numbers reflect absolute values $e . g$. duration of back pain, age)?

iii) The estimated time needed to complete the measure as identified by the author(s).

\section{d) Psychometric properties}

\section{i) Score range}

It is helpful to have an aggregate score for an outcome measure, together with an indication of its meaning. However this may not be within the context of normative data, since this is hard to establish in the psychosocial arena, and, as Bonder ${ }^{(17)}$ argues, is largely irrelevant. For example in the 'Back Home' study, it is not appropriate to describe a level of control of health in a person as 'normal', since this is meaningless. It is more useful that any final score is accompanied by interpretative commentary.

\section{ii) Reliability}

Reliability involves providing evidence of the value of an instrument - 'can it measure something in a reproducible fashion ${ }^{\text {(27). }}$. Chambers represents it by the following 'operation'(28):

\section{Measured Value $=$ True Value + Systematic Error + Random Error}

The usefulness of measurement depends on the extent to which the true value is obscured by the two types of error. Random error (or 'noise'), occurs when repeated measurements of some variable do not give the same values, but there is no systematic deviation from the true value, for example some intra-observer error ${ }^{(29)}$. Systematic error 
(bias) occurs when measurements deviate systematically (non-randomly) from the true value, for example sampling errors ${ }^{(29)}$. Errors can also be caused by the outcome measure itself e.g. ambiguous questions, unclear instructions, poor quality of printing / photocopying etc.

Reliability scores range between 0 (no reliability) and 1 (perfect reliability) ${ }^{(29)}$. Statistical correlations defining what level of reliability is acceptable differ considerably, ranging from correlations of $0.65-0.94^{(30)}$.

In self-report outcome measures there are predominantly two types of reliability: testretest reliability; and internal consistency or item-total correlation.

Test-retest reliability is a measure of stability and involves administering the same measure on two separate occasions, usually $2-14$ days apart ${ }^{(31)}$. It is necessary to select an appropriate time interval 'too long, and things may have changed, too short, and patients may remember their first response ${ }^{(31)}$. When measuring acute low back pain, with a rapid natural history of resolution, a short time interval of $2-3$ days would be appropriate, accepting that clients may have remembered their first response. If a longer time interval is allowed, the measurements are likely to be considerably different and therefore any attempts at gaining acceptable scores for test-retest reliability are likely to be unsuccessful. It is also important to retest any measures at comparable times throughout the day since there may be diurnal variations in perceived low back pain as natural recovery takes place. One possible explanation for such variations is that changes in disc hydration and height ${ }^{(32)}$ will affect the biomechanics of the spine, which in turn could affect the pain levels perceived and hence, the outcome of any measurements taken. Therefore trying to repeat measures at similar times will attempt to minimize this variation - a point observed throughout this research.

Internal consistency: If a measurement scale contains a number of items measuring the same dimension then it is expected that each score will correspond to scores of other comparable items. This is described as internal consistency, and results are based on a single administration of the measure. Cronbach's $\alpha$ test is often used to measure internal consistency and a minimum acceptable level has been quoted as 0.85 on all dimensions of the outcome measure ${ }^{(29)}$, although Streiner and Norman advocate Pearson productmoment correlation as the 'best coefficient to use ${ }^{\text {(33) }}$. They give the '... rule of thumb ... 
that an item should correlate with the total score above $0.20^{\prime}$ and suggest that items with lower correlations should be discarded ${ }^{(33)}$. With a natural history of resolution of acute low back pain within 4 weeks in $74 \%$ of patients ${ }^{(34)}$ (although as discussed in chapter two, this has recently been challenged $\left.{ }^{(35)}\right)$, nevertheless, the symptom is extremely variable during the early stages. Thus, internal consistency, which represents a snapshot, will be an important psychometric property, since for the reasons outlined above, testretest reliability is problematic in measuring acute back pain.

Streiner and Norman ${ }^{(27)}$ identify that since measures of internal consistency do not take account of inter-rater and intra-rater reliability, they can 'lead to an optimistic interpretation of the true reliability of the test'. However with self-report measures, inter-rater reliability is not an issue and so this 'optimistic interpretation' will be lessened.

\section{iii) Factor analysis}

This technique consists of clustering a number of individual items of information to identify common underlying characteristics or factors and, unlike item-total correlation, is specifically for multi-scale questionnaires ${ }^{(33)}$. It gives a statistical measure of how closely the individual items are interrelated and of the homogenicity (sic) of the underlying concept ${ }^{(36)}$. This can be useful in acute low back pain - a complex, multidimensional symptom, where the whole picture may be confusing. Streiner and Norman advocate that if the item 'loads on the "wrong factor", or two or more factors, then it is likely that it may be tapping something other than what the developer intended $^{\text {(33) }}$.

\section{iv) Validity}

The Chartered Society of Physiotherapy has published a document outlining standards for administering outcome measures in general, which should be adopted by chartered physiotherapists in clinical practise and research ${ }^{(37)}$. In this document, validity is defined as 'the extent to which a test measures what it is intended to measure'. Validity is a complex issue and hence a number of different forms of validity exist, including face, content, criterion, concurrent and construct validity. It has been argued that validity is the most important consideration when selecting an outcome measure ${ }^{(30)}$. In our review 
for the 'Back Home' study, validity considers the extent to which the outcome measures reviewed actually measure the dimensions of control, function, pain and anxiety, in clients with acute low back pain.

Face validity examines the credibility of the outcome measure and asks can we accept it at 'face value' or not $?^{(29)}$ For example, does the Anxiety Thermometer appear to be measuring anxiety?

Content validity is a judgement of 'completeness', i.e. 'whether the instrument samples all the relevant or important content or domains ${ }^{\text {(27), }}$, and not any extraneous elements ${ }^{(4)}$. This judgement is usually an holistic view of the scale, not of its individual items. Brooks states that 'the essential prerequisite for content validity is to be clear as to the purpose of the measure that has been developed'(29). According to Guyatt et al., quantitative testing of face and content validity are rarely attempted ${ }^{(1)}$, presumably because they are difficult concepts to quantify, and thus are not considered particularly robust. For example, when considering pain, it is difficult to quantify whether all the important domains have been addressed, including severity and nature of the pain.

Criterion validity: This is an example of a more robust form of validity. It has been defined as the '... correlation of a scale with some other measure ... ideally, a 'gold standard' which has been used and accepted in the field' ${ }^{(38)}$, that is already widely used and established in the field. It assumes that a definitive gold standard exists, which is not the case in acute low back pain. However, aspects of control, function, pain and anxiety have been measured against other well established measures in these fields, which although not ideal, is pragmatic.

When selecting outcome measures, if any modifications are made to the original instrument, then the revised version must be revalidated against the original (as the gold standard), to at least establish a measure of criterion validity in the revised version. This practice is not universally adopted, its omission undermines the psychometric properties of the 'revised' instrument.

In tables $4.2-4.5$, some instruments have been tested for criterion validity against measures of general health status, whilst others are tested against 'condition specific' measures for low back pain. In this case the term 'condition specific' is a misnomer as 'low back pain' is not a specific condition, but a symptom, as was discussed earlier in 
chapter two. Despite these limitations, criterion validity is an essential component in establishing the accuracy of outcome measures.

Concurrent validity is described as '... the degree to which a measure correlates with some important event or criterion occurring at the same time ${ }^{(39)}$. However, no guidelines are given for what constitutes 'approximately the same time', nor for the nature of the supporting evidence. This concept is not often quoted in the self-report literature, presumably because other supporting evidence is less 'objective' than other forms of data, such as physiological measurements.

Construct validity (also termed 'convergent validity' $\left.{ }^{(29)}\right)$ : Cole ${ }^{(30)}$ defines this as 'the degree to which the scores obtained concur with the underlying theories related to the content - the theoretical constructs'. The Task Force on Standards for Measurement in Physical Therapy ${ }^{(4)}$ state that the 'evidence for construct validity is through logical argumentation based on theoretical and research evidence'. Guion ${ }^{(i n}{ }^{38)}$ states that 'All validity is at its base some form of construct validity ... It is the basic meaning of validity'.

Its importance was addressed by Guyatt $e t a l^{(1)}$ who define construct validity as the most rigorous approach to establishing validity, since understanding the theoretical constructs will result in expectations about how an outcome measure should behave if it is valid. For example in this research, when measuring locus of control using the Multidimensional Health Locus of Control Scale, the ideas underpinning the locus of control construct, discussed in chapter two, contribute to the construct validity of this instrument.

\section{v) Sensitivity to change}

Sensitivity to change or 'responsiveness' expresses the ability of a measurement tool 'to detect change within subjects on the effects of treatment ${ }^{(40)}$. Kirshner and Guyatt ${ }^{(41)}$ state that it 'concerns the power of the index to detect a difference when one is present'.

If clients with acute low back pain are being followed-up in a longitudinal study, it is particularly challenging to find outcome measures which can accurately reflect the relatively large changes in symptoms which occur during the early stages of the natural history, and yet still detect changes when the rate of change decreases as the symptom 
becomes subacute or chronic. Sensitivity to change is therefore sometimes compromised. This consideration is likely to be less problematic in studies of clients with chronic low back pain, which is relatively more stable.

\subsection{The review}

Having established the criteria for reviewing the outcome measures according to the '3Ps'; purpose, practical aspects and psychometric properties, tables $4.2-4.5$ summarize attributes for measures of control, function, pain and anxiety respectively. Wherever possible, each measure was traced to its original source. The content of the tables is discussed in sections $4.8-4.11$.

\subsection{Control}

As Bowling observes in her review, trying to gauge clients' feelings of control over their life (including their health) is relevant to the 'outcome of illness and treatment because feelings of control may affect adherence to therapy and preventive health behaviour' ${ }^{(42)}$.

Within the literature many different dimensions of control have been cited, including locus of control, self-efficacy, perceived control, health beliefs, treatment beliefs etc., all of which aim to tap a slightly different phenomenon. Not surprisingly, selecting an appropriate outcome measure can therefore be complex. Bowling also comments that few 'condition-specific' control scales have been developed ${ }^{(42)}$, unlike functional outcome measures for example. Therefore a more global approach is necessary to reviewing these instruments.

Three outcome measures were chosen from the literature to review as potential instruments for the 'Back Home' study. Justification for this short-list is presented in section 4.12.1. The measures, in no particular order of merit, were:

- Rotter's Internal-External Locus of Control Scale (I-E) ${ }^{(43)}$

- Multidimensional Health Locus of Control Scale (M.H.L.C. $)^{(44)}$

- Perceived Control ${ }^{(45)}$ 
Table 4.2: Control

\begin{tabular}{|c|c|c|c|}
\hline Title & $\begin{array}{l}\text { Internal-External } \\
\text { Locus of Control } \\
\text { Scale (1966) }\end{array}$ & $\begin{array}{l}\text { Multidimensional } \\
\text { Health Locus of } \\
\text { Control Scale (1978) }\end{array}$ & $\begin{array}{l}\text { Perceived control } \\
\text { of diabetes scales } \\
(1984)\end{array}$ \\
\hline Author(s) & J B Rotter & $\begin{array}{l}\text { K A Wallston } \\
\text { BS Wallston } \\
\text { R DeVellis }\end{array}$ & $\begin{array}{l}\text { C Bradley } \\
\text { C R Brewin } \\
\text { D S Gamsu } \\
\text { J L Moses }\end{array}$ \\
\hline References & 42,43 & 42,44 & 45 \\
\hline $\begin{array}{l}\text { Purpose: } \\
\text { Discriminate } \\
\text { Predict } \\
\text { Evaluate }\end{array}$ & $\begin{array}{l}\checkmark \\
\checkmark \\
-\end{array}$ & $\begin{array}{l}\checkmark \\
\checkmark \\
-\end{array}$ & $\begin{array}{l}\checkmark \\
\checkmark \\
-\end{array}$ \\
\hline $\begin{array}{l}\text { Impairment } \\
\text { Functional limitation } \\
\text { Disability } \\
\text { Handicap }\end{array}$ & $\begin{array}{l}\checkmark \\
\checkmark \\
\checkmark \\
-\end{array}$ & $\begin{array}{l}\checkmark \\
\checkmark \\
\checkmark \\
-\end{array}$ & $\begin{array}{l}\checkmark \\
\checkmark \\
\checkmark \\
-\end{array}$ \\
\hline No. of items & 23 & 18 & $\begin{array}{l}6 \text { situations. } 7 \\
\text { items each }\end{array}$ \\
\hline $\begin{array}{l}\text { Style - self- } \\
\text { administered }\end{array}$ & - & $\checkmark$ & $\checkmark$ \\
\hline $\begin{array}{l}\text { Scale type - } \\
\text { categorical }\end{array}$ & $\checkmark$ & $\checkmark$ & $\checkmark$ \\
\hline $\begin{array}{l}\text { Level of } \\
\text { measurement }\end{array}$ & $\begin{array}{l}\text { Paired statements. } \\
\text { Forced choice }\end{array}$ & ordinal. 6 point & See text. 7 point \\
\hline $\begin{array}{l}\text { Estimated time to } \\
\text { complete (minutes) }\end{array}$ & 15 & - & - \\
\hline Score range & $0-23$ & Each subscale 6-36 & Each situation $0-18$ \\
\hline $\begin{array}{l}\text { Reliability: } \\
\text { internal consistency } \\
\text { test retest }\end{array}$ & $\begin{array}{l}\checkmark \\
\checkmark\end{array}$ & $\begin{array}{l}\checkmark \\
-\end{array}$ & $\begin{array}{l}\checkmark \\
-\end{array}$ \\
\hline $\begin{array}{l}\text { Validity: } \\
\text { construct } \\
\text { criterion } \\
\text { - general } \\
\text { - specific } \\
\text { content }\end{array}$ & $\begin{array}{l}\checkmark \\
\text { Social Desirability } \\
- \\
-\end{array}$ & $\begin{array}{l}\checkmark \\
\text { Marlowe-Crowne } \\
\text { Social Desirability } \\
\text { Levenson's I P \& } C^{\dagger} \\
\checkmark\end{array}$ & $\begin{array}{l}\checkmark \\
- \\
- \\
\checkmark\end{array}$ \\
\hline Factor analysis & $\checkmark$ & See text & $\checkmark$ \\
\hline Sensitivity to change & Not applicable & - & - \\
\hline
\end{tabular}

Key: $\dagger=$ Levenson's Internal, Powerful Others and Chance Scales 
The category 'sensitivity to change' is included for consistency with tables 4.3-4.5 in this chapter (although this issue was not addressed in any of the papers reporting the development of these instruments).

\subsubsection{Rotter's Internal-External Locus of Control Scale (I-E)}

Rotter's I-E Locus of Control Scale is based on the theory of internal versus external control, which was discussed in chapter two.

Rotter developed his scale with a group of college students ${ }^{(43)}$, and the scale is based on a series of paired items about control over life and health ${ }^{(42)}$. It aimed to allow a 'low degree of prediction of behaviour across a wide range of potential situations ${ }^{\text {(43) }}$, and the scale has 23 items, each is a forced choice, where respondents choose one of a pair of statements as indicative of their beliefs ${ }^{(42)}$. Rotter states that an earlier Likert format was discarded in favour of a forced-choice instrument in order to reduce correlations with the Marlowe-Crowne Social Desirability Scale ${ }^{(43)}$. Furthermore, using the Marlowe-Crowne Social Desirability Scale as criterion validity for Rotter's I-E Locus of Control Scale must be questioned, as the underlying theoretical constructs are not identical.

The scale has been widely used - indeed, it is reported to have been used in over half of all investigations of locus of control ${ }^{(42)}$. Nevertheless, the scale has not been subjected to rigorous testing ${ }^{(42)}$, although reliability is better documented than validity. It is not appropriate to consider 'sensitivity to change' in this instrument, since it measures trait control (as opposed to state control) and thus is not expected to change.

Rotter stipulates that the scale has to be administered by an interviewer who is trained to give and interpret personality measures and takes about 15 minutes to complete ${ }^{(42)}$. Scoring is by allocating one point for each external statement selected from the paired items. Thus it is not complex to complete or score.

\subsubsection{Multidimensional Health Locus of Control Scales (M.H.L.C.)}

This instrument was originally developed by Wallston et al. as a unidimensional measure of the extent of belief that health is determined by behaviour ${ }^{(42)}$. This scale was later developed to reflect the three dimensions of health locus of control beliefs that were discussed in chapter two - internal, powerful others and chance, and was hence deemed multidimensional. This critique focuses on the latter version. 
The 'new items' were developed by the authors ${ }^{(44)}$, although no details of their origin are included. The final scale (of which there are two parallel versions, forms A and B), contain 18 items, where each response involves a six point Likert scale ranging from 'strongly disagree' to 'strongly agree'. The scale was then tested on 125 people who were waiting at an international airport ${ }^{(44)}$, although nowhere do the authors consider any biases that might be inherent in this population.

Obvious potential biases may be evident with this sample, including socio-economic, cultural and behavioural biases. For example, the authors report that $90 \%$ of the population were white, $55 \%$ came from Tennessee and $74 \%$ had at least college education $^{(44)}$. Thus these findings are not considered generalizable of the general population. Such a population may be better able to represent the views of business people than those from lower socio-economic groups. In addition, by just consulting people who are willing to travel by air, the sample excludes people who are may not consider air travel to be safe etc. Such omissions may skew the findings.

The psychometric profile reported by Wallston et al. for the M.H.L.C. lacks details of test-retest reliability (subsequently established by Winefield) ${ }^{(46)}$. Furthermore, some debate exists about the factor structure, with authors supporting the concept of internal and powerful others as separate subscales, but with less coherence for the external chance subscale $e^{(42,46)}$.

\subsubsection{Perceived Control of Diabetes Scales}

This 'condition-specific' scale was developed for clients with diabetes (who require insulin), where previously; the more general locus of control scales had been unable to predict behaviour ${ }^{(45)}$. The scales were designed to examine attributions of responsibility for, and control over, both positive and negative outcomes concerning diabetes management. Although taking scales designed for people with diabetes may seem irrelevant to people with back pain, the general format of the scales had much to offer and no such scales were evident within the back pain literature. Hence their inclusion in this review.

The scale contains six descriptions of hypothetical events (relevant to clients with diabetes). Three of the events describe positive outcomes and three describe negative outcomes $^{(45)}$. For each hypothetical event, clients are asked to imagine that they have 
recently experienced the particular outcome and to write down its single most likely cause $^{(45)}$. Then they rate this cause on seven separate 7-point scales which are labelled Internality, Treatment, Externality, Chance, Perceived control, Medical control and Foreseeability. These scales are numbered from $0-6$, and are either ordinal or interval (for the reasons discussed in section 4.10.3).

Although the content and construct validity are reported, no criterion validity, sensitivity to change or test-retest reliability are reported. Before this instrument could be used in a study with clients experiencing acute low back pain, it needed to be tested and this is discussed in chapter five.

\subsection{Function}

Keith describes the construction of instruments to measure function as a "formidable undertaking' due to the number of potential functional activities ${ }^{(47)}$, and the number of different functional outcome measures in existence.

Firstly, it is essential to establish what is actually being measured? Is it a general concept such as quality of life, a specified activity e.g. sitting, an identified task such as getting dressed, or a combination of these?

When considering functional outcome measures for the 'Back. Home' study, eight measures were short-listed and their merits and limitations were considered. These eight scales were selected, either because they were the most commonly used within the appropriate published literature, or they were recommended as worthy of consideration by colleagues already working within this field. In no particular order of merit, the scales were:-

\section{- Low Back Outcome Score ${ }^{(48)}$}

- Waddell Disability Index ${ }^{(36)}$

- Oswestry Low Back Pain Disability Questionnaire ${ }^{(49)}$

- Disability Questionnaire (Roland Morris) ${ }^{(50)}$

- $\mathrm{SF} 36^{(51,52,53,54)}$

- Sickness Impact Profile (S.I.P.) ${ }^{(55,56,57)}$

- Nottingham Health Profile (N.H.P.) $)^{(58.59,60.61)}$

- Aberdeen Low Back Scale ${ }^{(62)}$ 
Table 4.3a: Function

\begin{tabular}{|c|c|c|c|c|}
\hline$\overline{\text { Title }}$ & $\begin{array}{l}\text { Aberdeen Low } \\
\text { Back Pain } \\
\text { Scale } \\
(1994)\end{array}$ & $\begin{array}{l}\text { Low Back } \\
\text { Outcome Score } \\
\text { (1991) }\end{array}$ & $\begin{array}{l}\text { Waddell } \\
\text { Disability } \\
\text { Index } \\
(1984)\end{array}$ & $\begin{array}{l}\text { Oswestry LBP } \\
\text { Disability } \\
\text { Questionnaire } \\
(1980)\end{array}$ \\
\hline Author(s) & $\begin{array}{l}\text { D A Ruta } \\
\text { A M Garratt } \\
\text { D Wardlaw } \\
\text { I T Russell }\end{array}$ & $\begin{array}{l}\text { CG Greenough } \\
\text { RD Fraser }\end{array}$ & $\begin{array}{l}\text { G Waddell } \\
\text { C J Main }\end{array}$ & $\begin{array}{l}\text { J C Fairbank } \\
\text { J Couper } \\
\text { J B Davies } \\
\text { J P O'Brien }\end{array}$ \\
\hline References & 62 & $48,62,63$ & $36,48,62,63$ & $\begin{array}{l}20,49,62,63 \\
64,65\end{array}$ \\
\hline $\begin{array}{l}\text { Purpose: } \\
\text { Discriminate } \\
\text { Predict } \\
\text { Evaluate }\end{array}$ & $\begin{array}{l}- \\
- \\
\checkmark\end{array}$ & $\begin{array}{l}\checkmark \\
- \\
\checkmark\end{array}$ & $\begin{array}{l}\checkmark \\
- \\
\checkmark\end{array}$ & $\begin{array}{l}\checkmark \\
- \\
\checkmark\end{array}$ \\
\hline $\begin{array}{l}\text { Impairment } \\
\text { Functional } \\
\text { limitation } \\
\text { Disability } \\
\text { Handicap }\end{array}$ & $\begin{array}{l}\checkmark \\
\checkmark \\
\checkmark \\
\checkmark\end{array}$ & $\begin{array}{l}\checkmark \\
\checkmark \\
\checkmark \\
\checkmark\end{array}$ & $\begin{array}{l}\checkmark \\
\checkmark \\
\checkmark \\
\checkmark\end{array}$ & $\begin{array}{l}2 \\
v \\
v \\
v \\
d\end{array}$ \\
\hline No. of items & 19 & 13 & 9 & 10 \\
\hline $\begin{array}{l}\text { Style - self- } \\
\text { administered }\end{array}$ & $\checkmark$ & $\checkmark$ & interview based & $\checkmark$ \\
\hline $\begin{array}{l}\text { Scale type - } \\
\text { categorical }\end{array}$ & $\checkmark$ & $\checkmark+$ VAS & $\checkmark$ & $\checkmark$ \\
\hline $\begin{array}{l}\text { Level of } \\
\text { measurement }\end{array}$ & $\begin{array}{l}\text { ordinal. } \\
\text { 3-6 point }\end{array}$ & ordinal. 4 point & dichotomous & $\begin{array}{l}\text { ordinal. } \\
6 \text { point }\end{array}$ \\
\hline $\begin{array}{l}\text { Estimated time to } \\
\text { complete (minutes) }\end{array}$ & - & - & 'quick' & $31 / 2-5$ \\
\hline Score range & $\begin{array}{l}169 \text { or } / 72 \\
\text { convert to } \%\end{array}$ & $0-75$ & $0-9$ & $\begin{array}{l}0-50 \\
\text { convert to } \%\end{array}$ \\
\hline $\begin{array}{l}\text { Reliability: } \\
\text { internal consistency } \\
\text { test retest }\end{array}$ & $\checkmark$ & - & $\begin{array}{l}\checkmark \\
-\end{array}$ & $\checkmark$ \\
\hline $\begin{array}{l}\text { Validity: } \\
\text { construct } \\
\text { criterion } \\
\quad \text { - general }\end{array}$ & $\checkmark$ & $\checkmark$ & $\checkmark$ & - \\
\hline $\begin{array}{l}\text { - specific } \\
\text { content }\end{array}$ & $\begin{array}{l}- \\
\checkmark\end{array}$ & $\begin{array}{l}\checkmark \text { Waddell DI, } \\
\text { PIR, \& } \\
\text { Oswestry } \\
-\end{array}$ & $\begin{array}{l}\checkmark \text { Oswestry } \\
\text { PII } \\
\checkmark\end{array}$ & $\begin{array}{l}- \\
\checkmark\end{array}$ \\
\hline Factor analysis & $\checkmark$ & - & $\checkmark$ & - \\
\hline Sensitivity to change & $\checkmark$ & - & $\checkmark$ & $\checkmark$ \\
\hline
\end{tabular}


Table 4.3b: Function

\begin{tabular}{|c|c|c|c|c|}
\hline Title & $\begin{array}{l}\text { SF 36 } \\
(1986) 1992\end{array}$ & $\begin{array}{l}\text { Nottingham } \\
\text { Health Profile } \\
\text { (1977) } 1981 \\
\end{array}$ & $\begin{array}{l}\text { Sickness } \\
\text { Impact Profile } \\
1976(1981) \\
\end{array}$ & $\begin{array}{l}\text { Disability } \\
\text { Questionnaire } \\
\text { (1983) }\end{array}$ \\
\hline Author(s) & $\begin{array}{l}\text { JE Ware } \\
\text { C Sherbourne }\end{array}$ & $\begin{array}{l}\text { (C Martini) } \\
\text { S M Hunt } \\
\text { J McEwen } \\
\text { S P McKenna }\end{array}$ & $\begin{array}{l}\text { M Bergner } \\
\text { B S Gilson } \\
\text { R A Bobbitt } \\
\text { W B Carter }\end{array}$ & $\begin{array}{l}\text { M Roland } \\
\text { R Morris }\end{array}$ \\
\hline References & $\begin{array}{l}51,52,53,54, \\
66\end{array}$ & $\begin{array}{l}20,58,59,60 \\
61,63,66,67\end{array}$ & $\begin{array}{l}20,55,56,57, \\
63,64,66,68\end{array}$ & $50,63,64,68$ \\
\hline $\begin{array}{l}\text { Purpose: } \\
\text { Discriminate } \\
\text { Predict } \\
\text { Evaluate }\end{array}$ & $\begin{array}{l}\checkmark \\
- \\
\checkmark\end{array}$ & $\begin{array}{l}\checkmark \\
\checkmark \\
v\end{array}$ & $\begin{array}{l}\checkmark \\
- \\
\checkmark\end{array}$ & $\begin{array}{l}\checkmark \\
\checkmark \\
\checkmark\end{array}$ \\
\hline $\begin{array}{l}\text { Impairment } \\
\text { Functional } \\
\text { limitation } \\
\text { Disability } \\
\text { Handicap } \\
\end{array}$ & $\begin{array}{l}\checkmark \\
\checkmark \\
\end{array}$ & $\begin{array}{l}\checkmark \\
\checkmark \\
v \\
v \\
\end{array}$ & $\begin{array}{l}\checkmark \\
\checkmark \\
\checkmark \\
\checkmark \\
\end{array}$ & $\begin{array}{l}2 \\
\checkmark \\
v \\
v \\
\end{array}$ \\
\hline No. of items & (20) 36 & (33) 38 & $235(146)(136)$ & 24 \\
\hline $\begin{array}{l}\text { Style - self- } \\
\text { administered }\end{array}$ & $\checkmark$ & $\checkmark$ & $\begin{array}{l}\checkmark \text { or } \\
\text { interviewer }\end{array}$ & $\checkmark$ \\
\hline $\begin{array}{l}\text { Scale type - } \\
\text { categorical }\end{array}$ & $\checkmark$ & $\checkmark$ & $\checkmark$ & $\checkmark$ \\
\hline $\begin{array}{l}\text { Level of } \\
\text { measurement }\end{array}$ & $\begin{array}{l}\text { ordinal. } \\
8 \text { multi-item }+ \\
1 \text { single item }\end{array}$ & $\begin{array}{l}\text { dichotomous. } \\
6 \text { sections }\end{array}$ & $\begin{array}{l}\text { dichotomous } \\
12 \text { categories }\end{array}$ & dichotomous \\
\hline $\begin{array}{l}\text { Estimated time to } \\
\text { complete (minutes) }\end{array}$ & $5-10$ & 'quick' & $20-30$ & 5 \\
\hline Score range & each scale as $\%$ & each section \% & convert to $\%$ & $0-24$ \\
\hline $\begin{array}{l}\text { Reliability: } \\
\text { internal consistency } \\
\text { test retest }\end{array}$ & $\checkmark$ & $\begin{array}{l}\checkmark \\
\checkmark\end{array}$ & $\begin{array}{l}\checkmark \\
\checkmark\end{array}$ & $\checkmark$ \\
\hline 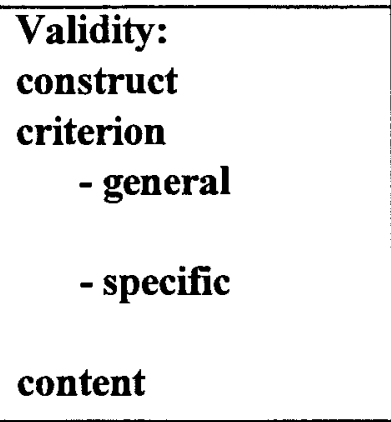 & $\begin{array}{l}\checkmark \\
\checkmark \text { MOS, GHRI, } \\
\text { MHI, SF20 } \\
- \\
\checkmark\end{array}$ & $\begin{array}{l}\checkmark \\
\checkmark \mathrm{GHQ} \\
- \\
\checkmark \\
\end{array}$ & $\begin{array}{l}\checkmark \\
\checkmark \text { NHIS, } \\
\text { ADL Index } \\
- \\
\checkmark\end{array}$ & $\begin{array}{l}\checkmark \\
\checkmark 6 \text { point pain } \\
\text { scale, S.I.P. } \\
\checkmark \text { Phys. signs } \\
\checkmark\end{array}$ \\
\hline Factor analysis & $\checkmark$ & $\begin{array}{l}(\text { version 1) } \checkmark \\
\text { version } 2- \\
\end{array}$ & $\checkmark$ & - \\
\hline Sensitivity to change & - & $\checkmark$ & $\checkmark$ & $\checkmark$ \\
\hline
\end{tabular}


Notes on tables $4.3 \mathrm{a}$ and $\mathrm{b}$

Where measures are developed from others and therefore different numbers of items are quoted, e.g. the N.H.P. from the Nottingham Health Index, the figures in bold indicate the value corresponding to the instrument being reviewed.

Key to tables $4.3 \mathrm{a}$ and $\mathrm{b}$ :

Waddell DI $=$ Waddell Disability Index

MHI $\quad=$ Mental Health Inventory

MOS $=$ Medical Outcomes Study

GHRI =General Health Rating Index

PIR / I = Physical Impairment Rating / Index

$$
\begin{array}{ll}
\text { GHQ } & =\text { General Health Questionnaire } \\
\text { NHIS } & =\text { National Health Interview Survey } \\
\text { ADL Index } & =\text { Katz's Index of ADL } \\
\text { Phys. signs } & =\text { Physical signs }
\end{array}
$$

\subsubsection{Low Back Outcome Score}

The Low Back Outcome Score was designed as a practical tool with clients attending an orthopaedic surgeon ${ }^{(48)}$. It consists of 13 items each with a choice of four answers, together with a visual analogue scale that measures pain. Responses are weighted, so that pain and 'activities' score more than treatments and rest required ${ }^{(48)}$.

The questionnaire has been described as emphasizing 'objective' questions such as returning to work $^{(63)}$, and the authors claim it is '... useful in the assessment of back function in the least disabled groups' ${ }^{(48)}$. They also report that 'subjective opinion was excluded', which is an interesting concept in a self-report measure.

In comparison with other functional outcome measures, the Low Back Outcome Scale is claimed to be more comprehensive than the Oswestry Disability Score, Waddell Disability Index or Physical Impairment Rating ${ }^{(48)}$. However, its psychometric profile is weakened by the lack of reported reliability and sensitivity to change, and as it includes items such as return to work, Pynsent argues that it may be subject to socio-economic influences such as the welfare system $^{(63)}$.

\subsubsection{Waddell Disability Index}

This instrument was developed to measure diminished capacity for everyday activities ${ }^{(36)}$, and can be "easily incorporated into routine assessments' ${ }^{\text {(36) }}$. It can be used together with a measure of objective physical impairment ${ }^{(36)}$.

The developmental work was with clients aged 20 - 55 years who reported at least a three month history of low back pain or sciatica ${ }^{(36)}$, referred to either an orthopaedic 
clinic or a regional Problem Back Clinic. The resulting questionnaire contains nine dichotomous questions, which the authors claim is 'quick' to administer ${ }^{(36)}$.

The measure has been described as poor at discriminating clients at the less disabled end of the scale ${ }^{(63)}$, which may render it less useful in primary care studies than hospital-based work. In addition, it does not address issues of employment, or details of sporting / social activities. However, omitting these items does render it less subject to the socioeconomic biases levied by Pynsent against the Low Back Outcome Score.

\subsubsection{Oswestry Low Back Pain Disability Questionnaire}

The Oswestry Low Back Pain Disability Questionnaire was one of the earliest outcome measures published, specifically designed for clients with chronic low back pain ${ }^{(49)}$.

The questionnaire consists of ten items, each with a choice of six answers and the authors claim that it takes $3 \frac{1}{2}-5$ minutes to complete ${ }^{(49)}$. They further claim that the items '... usually contain one idea and are simply worded ${ }^{\text {(49) }}$, but unfortunately this is not the case. Some responses contain multiple ideas e.g. 'I do not get dressed, wash with difficulty and stay in bed', which involves three separate activities. Furthermore, the questionnaire has no means of weighting, thus assuming all disabilities are equally disabling e.g. sleeping compared with sex life, which is questionable. In addition, the psychometric profile of the instrument is weakened by its poor reported validity.

The authors state that the questionnaire was designed to give 'the limitations of a patient's performance compared with that of a fit person ${ }^{(49)}$, although the measure has been criticized for only addressing disability based on pain, and therefore failing to allow for alternative causes of disability ${ }^{(64)}$, such as weakness, altered sensation etc.

When compared to the more recent Disability Questionnaire developed by Roland and Morris, the Oswestry Low Back Pain Questionnaire is reportedly less sensitive to changes in functional status of clients with minor disability, but more sensitive in clients with more severe disabilities ${ }^{\text {(Baker in 65) }}$. Hence it would seem more suited to a hospitalbased population than in primary care. 


\subsubsection{Disability Questionnaire}

This popular, condition-specific, functional outcome measure was developed by Roland and Morris. It has also been termed the 'St. Thomas's Questionnaire'(63) (sic). The measure evolved directly from the Sickness Impact Profile (S.I.P.), and was adapted to make it 'condition-specific' for low back pain ${ }^{(50)}$. It was then used with clients from a general practice population presenting with low back pain.

Among the attributes of this outcome measure are its simplicity, ease of completion and scoring. It consists of 24 dichotomous questions and the authors estimate that it takes five minutes to complete ${ }^{(50)}$. It also has a stronger psychometric profile than any of the functional outcome measures discussed so far. It is reported to function best with less severely affected clients ${ }^{(\mathrm{Baker} \text { in } 63)}$, making it a popular choice in primary care studies.

When validated against the S.I.P., the Disability Questionnaire was found to correlate well with the physical, but not the psychosocial dimension ${ }^{(68)}$, which suggests that it does not measure the psychosocial aspect of a limitation of function.

\subsubsection{SF36}

The SF36, which contains 36 items, is a shortened version of a battery of 149 health status questions developed and tested on a population of over 22000 clients, as part of the Medical Outcomes Study in the U.S.A. ${ }^{(53)}$ It is therefore a generalized measure of health status as opposed to a condition-specific functional outcome, and it is recommended for use as part of a more comprehensive portfolio of measures ${ }^{(53)}$.

The instrument contains eight distinct health status concepts and one item measuring self-reported health transition ${ }^{(69)}$, which results in a profile of scores. Interestingly, Brazier et al. claim that this makes it unsuitable for comparisons between treatments ${ }^{(54)}$, despite this criticism not being levied against other measures such as the Nottingham Health Profile (N.H.P.), which also does not have an overall score aggregate.

The SF36 reportedly takes $5-10$ minutes to complete ${ }^{(51)}$ and is thus shorter than the Sickness Impact Profile. It is also considered more sensitive to lower levels of dysfunction and disability than the N.H.P. ${ }^{(52)}$. The psychometric profile of the SF36 omits details of sensitivity to change ${ }^{(52)}$, and according to the authors, also omits 
measuring health distress, family functioning, sexual functioning, cognitive functioning and sleep disorders ${ }^{(51)}$. These may be important to clients with acute low back pain.

\subsubsection{Sickness Impact Profile}

The Sickness Impact Profile (S.I.P.) was developed as a measure of perceived health status, 'designed to be broadly applicable across types and severities of illness and across demographic and cultural subgroups' ${ }^{(55)}$. The scale was developed with a wide variety of client groups ${ }^{(57)}$, thus avoiding 'simple random sampling' ${ }^{(55)}$. Subsequently, the scale has been widely used, hence scores for many population groups are available for comparison $^{(67)}$.

The scale consists of 136 dichotomous items in twelve categories, and may be used either to give a single global health score, or subtotals for psychosocial and physical subscales ${ }^{(63)}$. It is also reportedly easy to complete: Hall et al. report the findings of a survey using the S.I.P., General Health Questionnaire and Rand Health-Insurance Battery, and in 160 questionnaires 'only $3 \%$ did not complete all the questions across the three scales' ${ }^{\text {(in } 67)}$. The scale does not record feelings, which is thought to minimize cultural bias ${ }^{(20)}$.

The main criticism levied against the S.I.P. is its length ${ }^{(55)}$, and therefore the time taken to complete and analyse it. Pynsent describes the measure as "needlessly complicated"(63), whilst Read comments it is tedious, repetitive, and necessitates a major commitment to interviewer training ('at least a week') ${ }^{(70)}$. Although it can be used without an interviewer as a self-report measure, administration by a trained interviewer is reportedly more reliable than self-administration ${ }^{(71)}$. In addition, Bowling claims that the S.I.P. does not measure positive functioning ${ }^{(67)}$, since it has been found to be more sensitive to declines in functioning than improvements ${ }^{(66)}$.

Specific to back pain, Pynsent comments that a large number of questions appear irrelevant to clients ${ }^{(63)}$, which may dilute its sensitivity. However, the S.I.P. has been used to develop the condition-specific Disability Questionnaire, which addresses this issue.

On balance, the usefulness of the S.I.P. is disputed. It has been described as one of the best instruments developed in U.S.A. ${ }^{(67)}$. Indeed, McDowell and Newell, (in Canada), 
commend its careful development, suggesting that it '.. is likely to become a standard against which to judge other methods' and they would have 'no hesitation in recommending its use in clinical and survey research' ${ }^{\prime(20)}$. Meanwhile, Pynsent says of the same tool that 'in general it is not recommended for use in the U.K.'(63).

\subsubsection{Nottingham Health Profile}

This instrument was developed in the U.K. and has been described as the 'only measure of perceived health which has been extensively tested and developed for use in Europe $^{(67)}$. It was originally conceived as a measure of quality of life and then developed as a population survey tool ${ }^{(60)}$.

There are two forms of this scale: the earlier Nottingham Health Index; and the Nottingham Health Profile ${ }^{(20)}$, which is more widely known. This discussion focuses on the latter.

The N.H.P. is a general health measure that relates to how people feel when they are experiencing various states of ill health ${ }^{(67)}$. It contains 38 dichotomous questions in six sections and is reported to take 10-15 minutes to complete ${ }^{(66)}$. Among its attributes, are its simplicity, and broad coverage $\mathrm{e}^{(20)}$.

The main criticisms of the N.H.P. appear to be its brevity, scoring ${ }^{(67)}$ and the issue that 'some milder forms of distress may not show up on the profile ${ }^{(60)}$. Furthermore, studies reporting sensitivity to change for this measure were for relatively extreme conditions such as pregnancy, fractures, heart and lung transplantation ${ }^{(67)}$. In practice, a large proportion of respondents with less severe symptomatic illness obtain scores of, or near zero and thus with an overall modal response of zero, the N.H.P. is reported to not discriminate for a substantial proportion of the adult population ${ }^{(59)}$. Therefore its use with clients with acute low back pain must be questioned, due to the natural history of resolution of this symptom. In addition, the N.H.P., unlike the S.I.P., reports feelings and is therefore, presumably more subject to cultural bias.

It is claimed that due to its brevity, the N.H.P. is a limited measure of function ${ }^{(67)}$, and is too short to assess the impact of a condition on quality of life ${ }^{(67)}$. The scoring has been described as 'cumbersome' as it gives a profile of $6 \operatorname{scores}^{(20)}$. McDowell states that a single score can be calculated ${ }^{(20)}$, although this does not appear to be consistent with the 
authors' original intentions. Bowling claims that each section does not represent just one dimension, and also reports that N.H.P. scores are not 'true' numbers but are obtained from a scaling technique, resulting in a highly skewed distribution of scores ${ }^{(67)}$.

The N.H.P. is further criticized for lacking an adequate index of mental distress ${ }^{(67)}$, which may be relevant when measuring acute low back pain.

\subsubsection{Aberdeen Low Back Scale}

The Aberdeen Low Back Scale was clinically-derived with clients presenting with low back pain in general practice, or referred as outpatients to orthopaedic, rheumatology and physiotherapy departments ${ }^{(62)}$, although unfortunately the authors do not report the detail of symptom duration for the study population.

The questionnaire contains nineteen items, each with a choice of between three and six answers. Psychometrically, the tool appeared sound, with reliability, validity and sensitivity to change examined ${ }^{(62)}$. The instrument was reported to have a stronger psychometric profile when compared with the Low Back Outcome Score, Waddell Disability Index and Oswestry Low Back Pain Disability Questionnaire ${ }^{(62)}$.

Unfortunately, the paper reporting the development of this outcome measure ${ }^{(62)}$ was still in press at the start of the 'Back Home' study, and so the scale had not been used in independent clinical trials. Therefore, it was less well established than other back pain instruments and it was only possible to discuss its potential merits and pitfalls with the authors themselves ${ }^{1}$, as no colleagues had personal experience of this measure.

\subsection{Pain}

Pain is a personal experience which has been described as a 'subjective phenomenon to which an outsider can have only limited access' ${ }^{\text {(72) }}$. It is a complex concept to attempt to measure as it is influenced by many factors, including culture, expectancy, meaning of pain $^{(73)}$, past experience, attention, stress, whether pain is occurring elsewhere in the body concurrently etc. In addition, it is not static as it changes over time. As it is a subjective experience, it is argued that it can only be measured by the person experiencing $\mathrm{it}^{(74)}$. 
Pain measurement scales are the commonest method of assessing client outcome in back pain ${ }^{(62)}$ : In 1994, Ruta et al. stated that 'at least 22 different scales have been reported in the literature ${ }^{\text {(62) }}$. Rainville et al. report that such scales have been shown to correlate poorly with measures of physical function ${ }^{(75)}$. Therefore pain measures need to be considered as a separate dimension from function.

When considering the dimension of pain, wherever possible, it is preferable to measure pain relief directly, since Huskisson states that measuring pain on two occasions and subtracting the second result from the first, introduces a mathematical artefact ${ }^{(74)}$. Nevertheless, attempting to measure pain still presents a challenge.

In order to address this challenge, four self-report instruments were chosen from the literature to review as potential outcome measures for the 'Back Home' study. In no particular order of merit, they were:

- McGill Pain Questionnaire ${ }^{(76,77)}$

- Visual analogue scale (V.A.S.) ${ }^{(78,79,80)}$

- Numerical rating scale (N.R.S.) ${ }^{(78)}$

- Simple descriptive scale (S.D.S.) or verbal rating scale (V.R.S.) ${ }^{(79,82,83)}$

In addition it was decided that participating clients would be asked to keep a pain diary, recording pain levels, medication and other events at four times during the day.

Key to table 4.4 :

PRI $\quad=\quad$ Pain Rating Index

NWC $=$ Number of words chosen

\footnotetext{
${ }^{1}$ With thanks to Danny Ruta and Andrew Garratt - personal communication 1994.
} 
Table 4.4: Pain

\begin{tabular}{|c|c|c|c|c|}
\hline Title & $\begin{array}{l}\text { McGill Pain } \\
\text { Questionnaire } \\
\text { (1975) }\end{array}$ & $\begin{array}{l}\text { Visual } \\
\text { Analogue Scale } \\
(1972)^{\dagger}\end{array}$ & $\begin{array}{l}\text { Numerical } \\
\text { Rating Scale } \\
\text { (date } \\
\text { unknown) } \\
\end{array}$ & $\begin{array}{l}\text { Simple } \\
\text { Descriptive } \\
\text { Scale / "VRS" } \\
(1948) \\
\end{array}$ \\
\hline Author(s) & R. Melzack & E.C. Huskisson ${ }^{\dagger}$ & unknown & Keele \\
\hline References & $20,63,76,77$ & $\begin{array}{l}20,64,72,78, \\
79,80\end{array}$ & 64,78 & $\begin{array}{l}79,81,82,83, \\
84\end{array}$ \\
\hline $\begin{array}{l}\text { Purpose: } \\
\text { Discriminate } \\
\text { Predict } \\
\text { Evaluate } \\
\end{array}$ & $\begin{array}{l}\checkmark \\
- \\
\checkmark\end{array}$ & $\begin{array}{l}\checkmark \\
- \\
\checkmark\end{array}$ & $\begin{array}{l}\checkmark \\
- \\
\checkmark\end{array}$ & $\begin{array}{l}\checkmark \\
- \\
\checkmark\end{array}$ \\
\hline $\begin{array}{l}\text { Impairment } \\
\text { Functional limitation } \\
\text { Disability } \\
\text { Handicap } \\
\end{array}$ & $\begin{array}{l} \\
- \\
- \\
-\end{array}$ & $\begin{array}{l}\checkmark \\
- \\
- \\
-\end{array}$ & $\begin{array}{l}\checkmark \\
- \\
- \\
-\end{array}$ & $\begin{array}{l}\checkmark \\
- \\
- \\
-\end{array}$ \\
\hline No. of items & $\begin{array}{l}\text { 61 descriptors } \\
(78) \text { in } 16(20) \\
\text { categories }\end{array}$ & 1 & 1 & 1 \\
\hline $\begin{array}{l}\text { Style - self- } \\
\text { administered }\end{array}$ & $\checkmark$ & $\checkmark$ & $\checkmark$ & $\checkmark$ \\
\hline $\begin{array}{l}\text { Scale type - } \\
\text { categorical }\end{array}$ & $\checkmark$ & continuous & $\checkmark$ & $\checkmark$ \\
\hline $\begin{array}{l}\text { Level of } \\
\text { measurement }\end{array}$ & 4 parts. Ordinal & Interval / ratio & see 4.10 .3 & $\begin{array}{l}\text { ordinal } 4 \text { point } \\
\text { ( } 5 \text { point })\end{array}$ \\
\hline $\begin{array}{l}\text { Estimated time to } \\
\text { complete (minutes) }\end{array}$ & $15-20$ & 0.5 & - & - \\
\hline Score range & $\begin{array}{l}\mathrm{PRI}=0-61(78) \\
\mathrm{NWC}=0-16 \\
(20)\end{array}$ & $\begin{array}{l}\text { usually } 0-100 \\
\text { (can use } 20 \\
\text { point grid) }\end{array}$ & $0-10(0-100)$ & $\begin{array}{l}\text { verbal } \\
\text { descriptors }\end{array}$ \\
\hline $\begin{array}{l}\text { Reliability: } \\
\text { internal consistency } \\
\text { test retest }\end{array}$ & - & - & - & - \\
\hline $\begin{array}{l}\text { Validity: } \\
\text { construct } \\
\text { criterion } \\
\quad \text { - general }\end{array}$ & $\checkmark$ & - & - & \\
\hline - general & & & & \\
\hline $\begin{array}{l}\text { - specific } \\
\text { content } \\
\end{array}$ & $\begin{array}{l}\text { V.A.S. } \\
\checkmark \\
\end{array}$ & S.D.S. \& N.R.S. & $\begin{array}{l}\text { V.A.S. \& } \\
\text { S.D.S. }\end{array}$ & $\begin{array}{l}\text { V.A.S. \& } \\
\text { N.R.S. }\end{array}$ \\
\hline Factor analysis & $\checkmark$ & - & - & - \\
\hline Sensitivity to change & $\checkmark$ & $\checkmark$ & $\checkmark$ & \\
\hline
\end{tabular}

Key: $\dagger=$ see text section 4.10 .2 


\subsubsection{McGill Pain Questionnaire}

The McGill Pain Questionnaire was developed in an attempt to specify the qualities of pain $^{(76)}$, which is particularly important in physiotherapy since trying to understand the nature of a person's pain usually forms part of every client interview.

The final questionnaire comprises sections relating to the client's diagnosis, medication, history of presenting complaint and accompanying symptoms. In addition there is a list of pain descriptors, which, although only part of the total instrument, will be the focus of this discussion.

Initially Melzack and Torgerson identified 102 pain descriptors from the literature and existing questionnaires ${ }^{(85)}$. These were subsequently reduced to 61 (and finally 78) words, that were arranged in 16 (and later 20) categories. The descriptors were sorted into three major classes - sensory, affective and evaluative ${ }^{(76)}$, and the quality and intensity of the descriptors was tested among people with different cultural, socioeconomic and educational backgrounds ${ }^{(63)}$, resulting in the development of the Pain Rating Index of the McGill Pain Questionnaire.

Perhaps the greatest strength of this instrument is that it attempts to address the nature of pain. It has also been described as having a good content validity ${ }^{(72)}$, and has a stronger psychometric profile than the other pain outcomes considered in this research.

However, on the down-side, the instrument is considered time-consuming to complete and complex to score ${ }^{(72)}$, and a subsequent short-form has been developed ${ }^{(86)}$. In addition, some descriptors may be beyond a client's vocabulary ${ }^{(76)}$ (and this certainly was the case in our research, especially with descriptors such as 'lancinating').

\subsubsection{Visual analogue scale (V.A.S.)}

Visual analogue scales have been used in psychology since the '... early part of this century to measure such unmeasurables as personality, depression and sleep ${ }^{\text {(79) }}$. In 1964 Clarke and Spear used a V.A.S. to measure well-being ${ }^{(79)}$, and it is not clear who first adapted the V.A.S. to measure pain. There is no doubt that Huskisson has been a '... leading exponent of this approach'(20), although Woodforde and Merskey also cite adapting Clarke and Spear's scale in $1972^{(87)}$. 
The V.A.S. is a straight line that represents the continuum of the symptom to be rated ${ }^{(20)}$. It is usually a ten centimetre line anchored at each end with statements representing extremes of the dimension being measured ${ }^{(72)}$. Variations include vertical scales ${ }^{(80)}$, curvilinear and graded scales ${ }^{(88)}$ and the addition of descriptive terms placed at intervals along the line (when it is known as a graphic rating scale) ${ }^{(78)}$.

Among its merits are that the V.A.S. is reported to be '... potentially highly responsive ${ }^{\text {(72) }}$ and can produce interval / ratio level data ${ }^{(72)}$. It is also quick to use, with estimates that it takes only about 30 seconds to complete ${ }^{(20,78)}$, whilst Cole et al. suggest that the explanation and administration of the task takes ' $<5$ min' and the scoring ' $<1 \mathrm{~min}$.' ${ }^{\text {(64) }}$

Its weaknesses are that people find simple descriptive scales easier than V.A.S. ${ }^{(79)}$. Huskisson himself states that '... in a group of patients ... 7\% were unable to complete a visual analogue pain scale on the first occasion after a single adequate explanation of the

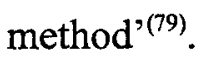

The content validity of the V.A.S. has been described as poor owing to its unidimensionality ${ }^{(72)}$, although $\mathrm{Sim}$ and Waterfield suggest using two or more V.A.S. may counter this shortcoming ${ }^{(72)}$. By contrast, Cole et al. report the content validity to be high as the V.A.S. directly measures intensity of pain ${ }^{(64)}$. However, intensity of pain is only one aspect of pain and thus Cole et al. 's commendations may be considered overly generous. As a practical point, repeated photocopying of this scale may distort its $\operatorname{accuracy}^{(64)}$.

\subsubsection{Numerical rating scales (N.R.S.)}

The N.R.S. is a numeric scale developed to measure the magnitude or intensity of pain ${ }^{(64)}$, which can be administered either verbally or in written form. The original author is unknown $^{(64)}$, and a number of variations exist e.g. 11 point scale (scoring $\left.0-10\right)^{\left({ }^{(C o l e)}\right.}$ and a 101 point scale (scoring $0-100)^{(89)}$.

The scale is quick to use - Cole et al. suggest that explanation and administration takes five minutes ${ }^{(64)}$.

However, the psychometric profile of the N.R.S. is weak. Similar to the V.A.S., Cole et al. suggests that the content validity is high, although for the same reasons as identified in 4.10.2, this may be overly optimistic. 
In addition, there is some dispute about the level of measurement of the scale. Some authors state that it is ordinal ${ }^{(72)}$, although the basis for this is not clear. Presumably it could be argued that what is a score of 20 for example to one person, does not necessarily mean the same for another person. In contrast, when the difference between responses is known in a scale, Streiner and Norman deem it to be interval ${ }^{(90)}$, and some may even argue that if there is a meaningful zero, for example 'no pain', then by inference the scale could even be considered ratio.

\subsubsection{Simple descriptive scale or verbal rating scale (S.D.S.)}

The S.D.S. is a hierarchical adjectival $\operatorname{scale}^{(72)}$ that has been described as the most fundamental form of pain measurement scale ${ }^{(81)}$.

Keele originally described a four-point scale, grading pain as slight, moderate, severe and agonising $^{(\mathrm{in} 79)}$. The term 'mild' is often used instead of 'slight' and since agonizing pain is rare, this grade is not often used ${ }^{(79)}$. Another variation is 'nil, mild, moderate, severe, very severe $^{(81)}$.

The S.D.S. is a 'useful standard method' of measuring pain owing to its simplicity ${ }^{(79)}$. It is quick and easy to complete although Linton and Götestam suggest it may be more difficult to fill out than the V.A.S. ${ }^{(82)}$. However, their scale, although described as a verbal rating scale, was more detailed and complex than Keele's original scale, with every point being behaviourally defined.

The S.D.S. has been criticized for forcing an individual to translate a feeling into words which '... does not express exactly what a person is experiencing' as the same word does not necessarily mean the same thing to each person ${ }^{(83)}$. In addition, the use of this scale for comparative purposes is limited by its lack of sensitivity for detecting relatively small changes ${ }^{(81)}$. Between the limits of 'agonizing pain' and 'no pain' there are only three

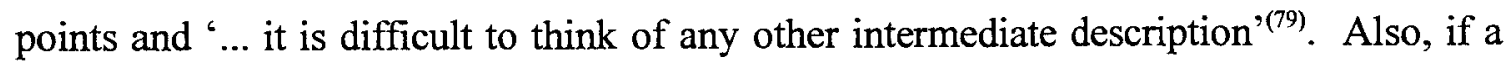
person reports 'slight pain', they only have one possible grade of improvement complete relief ${ }^{(79)}$.

The S.D.S. has frequently been compared with the V.A.S. ${ }^{(81,82,83.84)}$. Machin et al., in a single-blind randomized trial of transcutaneous nerve stimulation (T.E.N.S.) versus a placebo control of mock T.E.N.S. in clients with low back pain, reported that the verbal 
descriptive scale and V.A.S. appeared to be measuring different aspects of pain, and conclude that the two scales are not interchangeable ${ }^{(84)}$. Consequently, they suggest using both scales in randomized trials ${ }^{(84)}$.

\subsection{Anxiety}

It has long been argued that pain and anxiety are closely related and Waddell states that treatment of acute pain should deal with anxiety as this can help to reduce pain ${ }^{(91)}$.

Therefore it was necessary to select an instrument which would measure this dimension. Anxiety is usually considered as '... a complex reaction or response - a transitory state or condition ... that varies in intensity and fluctuates over time'(92). This is termed 'state anxiety' and a positive finding may be interpreted as a person being anxious now ${ }^{(92)}$. However, the term anxiety may also be used to refer to a personality trait - i.e. the extent to which someone is an anxious person ${ }^{(92)}$. This is known as 'trait anxiety'(92).

When studying clients with acute low back pain, understanding state anxiety is particularly important. It might help give information such as whether an intervention affects clients' levels of anxiety, or help to establish how their anxiety level changes as their back pain episode resolves.

Therefore, in selecting an appropriate outcome measure, six instruments were short-listed from the literature as potential measures of anxiety for the 'Back Home' study. Justification for this short-list is presented in section 4.12.4. The measures, in no particular order of merit were:

- State-Trait Anxiety Inventory (S.T.A.I.) $)^{(93)}$

- Short form of the State Scale of Spielberger S.T.A.I. ${ }^{(94)}$

- Hospital Ânxiety and Depression Scale ${ }^{(95,96)}$

- Leeds Scales ${ }^{(12)}$

- Anxiety Thermometer ${ }^{(97)}$

- Multiple Affective Adjective Check List (M.A.A.C.L. $)^{(98)}$ 
Table 4.5a: Anxiety

\begin{tabular}{|c|c|c|c|}
\hline Title & $\begin{array}{l}\text { State-Trait Anxiety } \\
\text { Inventory } \\
\text { (1970) } 1983\end{array}$ & $\begin{array}{l}\text { Short form of the } \\
\text { State Scale of } \\
\text { Spielberger S.T.A.I. } \\
(1992)\end{array}$ & $\begin{array}{l}\text { Hospital Anxiety } \\
\text { and Depression } \\
\text { Scale } \\
(1983)\end{array}$ \\
\hline Author(s) & $\begin{array}{l}\text { C.D. Spielberger } \\
+ \text { collaborators }\end{array}$ & $\begin{array}{l}\text { T.M. Marteau } \\
\text { H Bekker }\end{array}$ & $\begin{array}{l}\text { A.S.Zigmond } \\
\text { R.P.Snaith }\end{array}$ \\
\hline References & 93,94 & 94 & 95,96 \\
\hline $\begin{array}{l}\text { Purpose: } \\
\text { Discriminate } \\
\text { Predict } \\
\text { Evaluate }\end{array}$ & $\begin{array}{l}\checkmark \\
\checkmark \\
\checkmark\end{array}$ & $\begin{array}{l}\checkmark \\
- \\
-\end{array}$ & $\begin{array}{l}\checkmark \\
\checkmark \\
-\end{array}$ \\
\hline $\begin{array}{l}\text { Impairment } \\
\text { Functional limitation } \\
\text { Disability } \\
\text { Handicap } \\
\end{array}$ & $\begin{array}{l} \\
- \\
- \\
- \\
\end{array}$ & $\begin{array}{l}\checkmark \\
- \\
- \\
-\end{array}$ & $\begin{array}{l}\checkmark \\
- \\
- \\
-\end{array}$ \\
\hline No. of items & 40 & 6 & $7+7$ \\
\hline $\begin{array}{l}\text { Style }- \text { self- } \\
\text { administered }\end{array}$ & $\checkmark$ & $\checkmark$ & $\checkmark$ \\
\hline $\begin{array}{l}\text { Scale type - } \\
\text { categorical }\end{array}$ & $\checkmark$ & $\checkmark$ & $\checkmark$ \\
\hline $\begin{array}{l}\text { Level of } \\
\text { measurement }\end{array}$ & ordinal & ordinal. 4 point & ordinal. 4 point \\
\hline $\begin{array}{l}\text { Estimated time to } \\
\text { complete (minutes) }\end{array}$ & $10-20$ (total) & - & - \\
\hline Score range & $20-80$ for each scale & $6-24$ & $\begin{array}{l}0-21 \text { each } \\
\text { subscale }\end{array}$ \\
\hline $\begin{array}{l}\text { Reliability: } \\
\text { internal consistency } \\
\text { test retest }\end{array}$ & $\begin{array}{l}r \\
r \\
\end{array}$ & $\begin{array}{l}\checkmark \\
- \\
\end{array}$ & $\begin{array}{l}\checkmark \\
- \\
\end{array}$ \\
\hline $\begin{array}{l}\text { Validity: } \\
\text { construct } \\
\text { criterion }\end{array}$ & $\checkmark$ & - & $\checkmark$ \\
\hline - general & $\checkmark$ Personality tests & & - \\
\hline $\begin{array}{l}\text { - specific } \\
\text { content }\end{array}$ & $\begin{array}{l}\text { I.P.A.T., T.M.A.S., } \\
\text { A.A.C.L. } \\
\checkmark\end{array}$ & $\begin{array}{l}\text { S.T.A.I. } \\
- \\
\end{array}$ & $\begin{array}{r}- \\
\checkmark \\
\end{array}$ \\
\hline Factor analysis & $\checkmark$ & - & - \\
\hline Sensitivity to change & $\checkmark$ & $\checkmark$ & - \\
\hline
\end{tabular}

Key:
I.P.A.T = IPAT Anxiety Scale
A.A.C.L. $=$ Affect Adjective Check List
T.M.A.S. = Taylor Manifest Anxiety Scale
S.T.A.I. $=$ State Trait Anxiety Inventory 
Table 4.5b: Anxiety

\begin{tabular}{|c|c|c|c|}
\hline Title & $\begin{array}{l}\text { Leeds Scales } \\
(1976)\end{array}$ & $\begin{array}{l}\text { Anxiety } \\
\text { Thermometer } \\
\text { (1989) }\end{array}$ & $\begin{array}{l}\text { Multiple Affective } \\
\text { Adjective Checklist } \\
\text { (1964) }\end{array}$ \\
\hline Author(s) & $\begin{array}{l}\text { R.P. Snaith } \\
\text { G.W.K. Bridge } \\
\text { M. Hamilton }\end{array}$ & $\begin{array}{l}\text { Unknown (see text } \\
4.11 .5\end{array}$ & $\begin{array}{l}\text { M. Zuckerman } \\
\text { B. Lubin } \\
\text { L. Vogel } \\
\text { E. Valerius }\end{array}$ \\
\hline References & 12 & 97 & $98,(99)$ \\
\hline $\begin{array}{l}\text { Purpose: } \\
\text { Discriminate } \\
\text { Predict } \\
\text { Evaluate } \\
\end{array}$ & $\begin{array}{l}\checkmark \\
- \\
-\end{array}$ & $\begin{array}{l}\checkmark \\
- \\
\checkmark\end{array}$ & $\begin{array}{l}\checkmark \\
- \\
- \\
\end{array}$ \\
\hline $\begin{array}{l}\text { Impairment } \\
\text { Functional limitation } \\
\text { Disability } \\
\text { Handicap } \\
\end{array}$ & $\begin{array}{l}\checkmark \\
- \\
- \\
- \\
\end{array}$ & $\begin{array}{l}\checkmark \\
- \\
- \\
- \\
\end{array}$ & $\begin{array}{l} \\
- \\
- \\
- \\
\end{array}$ \\
\hline No. of items & $\begin{array}{l}\text { (22) } 4 \times 6 \text { items in } \\
\text { each scale }\end{array}$ & 1 & 132 adjectives \\
\hline $\begin{array}{l}\text { Style }- \text { self- } \\
\text { administered }\end{array}$ & $\checkmark$ & $\checkmark$ & $\checkmark$ \\
\hline $\begin{array}{l}\text { Scale type - } \\
\text { categorical }\end{array}$ & $\checkmark$ & $\checkmark /-$ & $\checkmark$ \\
\hline $\begin{array}{l}\text { Level of } \\
\text { measurement }\end{array}$ & ordinal. 4 point & $\begin{array}{l}10 \text { point Likert or } \\
\text { continuous }\end{array}$ & nominal \\
\hline $\begin{array}{l}\text { Estimated time to } \\
\text { complete (minutes) }\end{array}$ & - & - & - \\
\hline Score range & 0 -18 each scale & $1-10$ & - \\
\hline $\begin{array}{l}\text { Reliability: } \\
\text { internal consistency } \\
\text { test retest }\end{array}$ & $\begin{array}{l}\checkmark \\
- \\
\end{array}$ & 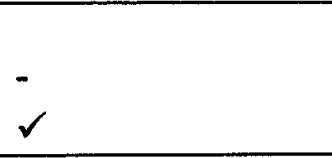 & - \\
\hline $\begin{array}{l}\text { Validity: } \\
\text { construct } \\
\text { criterion } \\
\quad \text { - general }\end{array}$ & - & - & - \\
\hline -gentral & & & \\
\hline $\begin{array}{l}\text { - specific } \\
\text { content }\end{array}$ & $\begin{array}{l}\text { Hamilton Rating } \\
\text { Scales } \\
- \\
\end{array}$ & $\begin{array}{l}\text { S.T.A.I. } \\
- \\
\end{array}$ & - \\
\hline Factor analysis & - & - & - \\
\hline Sensitivity to change & - & - & $\checkmark$ \\
\hline
\end{tabular}




\subsubsection{State-Trait Anxiety Inventory (S.T.A.I.)}

The S.T.A.I. is reported to be one of the most frequently used measures of anxiety in applied psychology research ${ }^{(94)}$. It is designed to evaluate how respondents feel '... right now, at the moment' (i.e. state anxiety) and how people generally feel (i.e. trait anxiety $)^{(93)}$. It was developed for use in high school, college students and adults ${ }^{(93)}$.

The S.T.A.I. consists of two questionnaires of 20 items each, measuring state and trait anxiety. There are two versions of the scale - form X, published in 1970 and form $Y$, in 1983. The latter, in which $30 \%$ of the original form $\mathrm{X}$ items were replaced, is deemed 'purer', with a firmer basis for discriminating between feelings of anxiety and depression, stronger psychometric profile and improved factor structure ${ }^{(93)}$.

The scale is considered reliable and sensitive ${ }^{(94)}$, indeed it has the strongest psychometric profile of all the anxiety instruments reviewed.

The main disadvantage with this instrument is its length, which Marteau and Bekker state is a barrier to its use when time is limited ${ }^{(94)}$. Spielberger says that college students generally require about ten minutes to complete the S.T.A.I. while 'less educated or emotionally disturbed persons' may require approximately twenty minutes to complete the scales ${ }^{(93)}$.

\subsubsection{Short form of the State scale of the S.T.A.I.}

This questionnaire was developed as a short form of the state scale of the S.T.A.I. for use in settings where time constraints preclude the use of the full form ${ }^{(94)}$. It consists of six items, each with a choice of four ordinal responses.

Initial developmental work was undertaken with a sample of pregnant women, medical and nursing students ${ }^{(94)}$.

The questionnaire is brief and considered likely to maximize response rates and minimize the number of response errors and unanswered items '... thus improving validity and generalizability of any findings'(94). A brief questionnaire will also reduce the time taken to complete and score $\mathrm{it}^{(94)}$.

The scale only considers state anxiety (and not trait) and has only been validated against the S.T.A.I., with which (not surprisingly), it correlates well ${ }^{(94)}$. (From experience of 
using this scale, clients were often confused that three items were positive and the other three were negative, which often resulted in errors in completion.)

\subsubsection{Hospital Anxiety and Depression Scale (H.A.D.)}

The H.A.D. Scale was developed to provide clinicians and scientists with a practical tool for identifying and quantifying the two most common forms of psychological disturbance in medical patients ${ }^{(100)}$ - anxiety and depression. The authors state that the scale was devised '... after a request from a colleague for a simple device to separate depressive disorder that can be treated with antidepressant drugs from sadness, demoralisation, and grief $^{(101)}$. The developmental work was conducted in general medical outpatient clinics on adults 'who suffered from a wide variety of complaints and illnesses $^{(95)}$.

The scale consists of 14 items (seven anxiety and seven depression), each with a choice of four ordinal answers. It is reported to be easy to administer and is well accepted ${ }^{(100)}$. Since severely psychopathological symptoms are not covered, this is thought to improve acceptability and make the scale '... more sensitive to mild forms of psychiatric disorders ${ }^{{ }^{(100)}}$. Herrmann reports that the scale can be completed in 2-6 minutes and with practise, scored in one minute ${ }^{(100)}$.

The psychometric profile reported in the original article ${ }^{(95)}$ is limited, with very sparse data on reliability and validity ${ }^{(100)}$. Subsequent work has been done to develop the instrument and factor analysis, construct validity, criterion validity (with S.T.A.I., and Beck Depression Inventory), and sensitivity to change are reported ${ }^{(100)}$.

Although the authors claim to have eliminated '... all items relating both to emotional disorder and physical disorder, e.g. 'dizziness' and 'headaches'(95), some physical components are still present which may be influential in measuring back pain. For example one item reads: 'I can sit at ease and feel relaxed', which contains the physical component of sitting and the psychosocial component of feeling relaxed. If a person has acute low back pain, physically sitting may be problematic irrespective of whether they feel relaxed. This issue has not been recognized in the literature surrounding the use of this scale. 


\subsubsection{Leeds Scales for the Self-Assessment of Anxiety and Depression}

The Leeds Scales are four scales covering the range of common symptoms of depressive illness and anxiety states, that have been developed '... based upon the Wakefield SelfAssessment Inventory with added items ${ }^{\prime(12)}$. The scales are:

- Leeds Self-Assessment of Depression Specific Scale (S.A.D. Specific)

- Leeds Self-Assessment of Anxiety Specific Scale (S.A.A. Specific)

- Leeds Self-Assessment of Depression General Scale (S.A.D. General)

- Leeds Self-Assessment of Anxiety General Scale (S.A.A. General)

The specific scales aim to measure the severity of endogenous depressive illness and anxiety neurosis, whereas the general scales are deemed suitable for assessment of severity of depression or anxiety in clients who have not received a formal diagnosis of endogenous depression or anxiety neurosis ${ }^{(12)}$.

Initial developmental work was with clients who were experiencing a depressed phase of manic depressive psychosis, depressive neurosis, anxiety neurosis, phobic neurosis, hypochondriacal neurosis, or obsessive-compulsive neurosis, together with hospital and Prison Training College staff ${ }^{(12)}$.

The resultant scales each contain six items, which have a choice of four responses. The authors argue that it is not possible to cover all the symptoms of depressive illness and anxiety states without making the scale unwieldy by its length, which limits the content validity of the scales, although criterion validity was established against the Hamilton Depression and Anxiety Rating Scales ${ }^{(12)}$.

\subsubsection{Anxiety thermometer}

The origins of the anxiety thermometer are unclear in the literature and the scale is reported to be '... either a continuous or a 10- (or 11-) point Likert-type scale on which subjects are asked to rate their anxiety feelings at a particular moment ${ }^{3(97)}$. Thus the scale could be either continuous or categorical.

The anxiety thermometer is reported to be a quick way to measure state-anxiety ${ }^{(97)}$, that has been described as a valuable instrument for measuring an ego-threatening real-life situation, such as an examination ${ }^{(97)}$. 
A major disadvantage of the scale was that Houtman and Bakker reported in 1989 that '... no psychometric data seem to be available'(97). Therefore, they completed a validation study with students undertaking a written examination, using the S.T.A.I. to establish the criterion validity. In this study they used a $10 \mathrm{~cm}$ continuous scale with end markers 'not

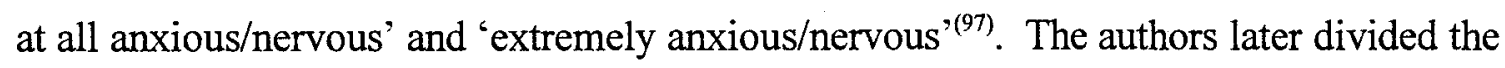
scale into equal parts which they scored from 1 to 10 . It is not clear why someone who reported being 'not at all anxious/nervous' would therefore still achieve a score of 1 .

\subsubsection{Multiple Affect Adjective Check List (M.A.A.C.L.)}

The M.A.A.C.L. was designed to measure day to day changes in three affects: anxiety, depression and hostility ${ }^{(98)}$, although it is not clear why these three affects were chosen.

The scale consists of 132 adjectives - 21 anxiety, 40 depression, 28 hostility (and others $)^{(98)}$, but the authors do not report exactly how the scale is used or scored, or indeed practical issues such as the time it takes to complete.

Developmental work involved 'neuropsychiatric inpatients's(98) plus psychology students (in various experimental situations including a hypnotic trance, seeing a stressful film, and under threat of a surprise exam $)^{(98)}$. Despite comprehensive details of the interventions, how these impacted on the final measure is unclear.

The psychometric profile of the outcome measure is poor, with no underlying theoretical constructs reported, or other means of validity.

\subsection{Selecting measures for the 'Back Home' Study}

As discussed in sections 4.5 and 4.6, selecting outcome measures for a research study is complex. It is essential to not only consider the study design, but also the purpose, practicalities and psychometric properties of any measurements that are chosen.

Another consideration is whether the outcome measure was developed for use in a population with back pain, a general population or a group of clients experiencing other diseases where the measure has subsequently been revised or validated for a population with back pain. This will affect the face validity of the instrument. This factor is in addition to the purpose, practical issues and psychometric properties of the outcome measure. If participants were experiencing back pain, were their symptoms from an 
acute exacerbation, subacute, chronic or recurrent episode? Many researchers do not appear to address this issue, since as discussed in section 2.2, acute and chronic low back pain differ in many ways other than simply duration.

The outcome measures chosen for the 'Back Home' Study are identified in the tables by bold type. Whilst the purpose and psychometric properties of reliability, validity and sensitivity to change, were essential considerations, practical issues became of paramount importance because of the number of outcome measures chosen for this study and the client population under review. (This is further discussed in chapters five and nine.) Clients with acute low back pain were not physically able to sit for hours completing questionnaires, and the time-frame necessitated the first assessment being completed within two working days of the client visiting their G.P., when they usually still had considerable discomfort. Indeed, at times, clients were not able to physically sit at all, to complete the questions due to the severity of their symptoms. Therefore, when considering the appropriate criteria for the outcome measures, each scale was reviewed using all the criteria in the systematic framework, but scale length and psychometric properties particularly influenced the final selection.

It was also vital to minimize 'respondent burden' when numerous outcome measures are administered. Having coined this phrase, Brooks identifies refusal rates, missing responses and administration time as indicators of this phenomenon ${ }^{(29)}$. However, since acute low back pain is multidimensional, many variables needed to be ascertained. Getting the balance between collecting sufficient data to ensure thorough analysis whilst avoiding or minimizing 'respondent burden' is a delicate one. On reflection, perhaps we asked too much of our clients at assessment 2 (two weeks), by administering 11 selfreport questionnaires. Certainly on occasions, clients who were either: more careful in their responses; had slower reading rates; were finding it difficult to concentrate; or those who were still in considerable discomfort, took about an hour to complete the questionnaires. The appropriateness of this is questionable.

\subsubsection{Selection of control measures}

For the 'Back Home' study (discussed in chapter five), it was decided that locus of control was an important factor to consider in evaluating an educational intervention. Rotter's I-E Scale was initially considered but there were two main barriers to its use. 
Firstly some of the items in this scale appeared somewhat remote to health perceptions, covering topics such as wars, children's behaviour etc. In addition, the unidimensional nature of this instrument, i.e. choosing either an internal or external statement, has been superceded by the proposed multidimensional nature (discussed in chapter two).

Having decided that the dimension of interest was locus of control in relation to health, it was then necessary to consider the study design. Since all participating clients had been recruited into the 'Back Home' study by their G.P., they would all have consulted their G.P. for this episode of acute low back pain. Therefore, it was important to establish the influence of 'powerful others', including the G.P., as perceived by the client. This steered our search for an appropriate instrument away from outcome measures such as the original Health Locus of Control Scale ${ }^{(44)}$ and the Recovery Locus of Control Scale $^{(102)}$.

At the time of searching, the M.H.L.C. Scale was considered the most appropriate instrument for our purpose, although its development was apparently biased (section 4.8.2) and the psychometric profile had some limitations. The six-point ordinal responses (in the form of a Likert scale) were considered likely to be more sensitive than forced choice paired statements, such as in Rotter's I-E Scale. The M.H.L.C. Scale had been widely used and it contained a focus on preventive health behaviours, which was deemed important in clients with acute low back pain, owing to the rapid natural history of resolution of this symptom, but likelihood of subsequent recurrent episodes, which was discussed in chapter two. Therefore perceptions of control in relation to preventing future episodes was an important factor to assess. Interestingly, early in our work, we learned that Wallston et al. had developed a 'condition-specific' form $\mathrm{C}$ of the scale, which is discussed in chapter six. This revised version was also used in this research.

In addition, it was decided to modify the Perceived Control of Diabetes Scales for use with a back pain population and this is discussed in chapter five.

\subsubsection{Selection of function measure}

When it came to selecting a functional outcome measure, a wide variety were available and five 'condition-specific' and three general health measures were short-listed. 
Eventually the Aberdeen Low Back Pain Scale was chosen as it was designed for a general practice population (unlike the Low Back Outcome Score, Oswestry Low Back Pain Disability Questionnaire and Waddell Disability Index). It demonstrated a stronger psychometric profile than these three measures (in direct comparison) and was thought to be potentially discriminating of clients at the less disabled end of the scale, than either the Waddell Disability Index (where this issue has been acknowledged ${ }^{(63)}$ ), or the N.H.P. where the modal response is zero ${ }^{(59)}$. Furthermore, the ordinal responses, with a choice of 3-6 answers, were considered theoretically more sensitive to detect smaller changes than dichotomous scales such as Roland and Morris' Disability Questionnaire and the N.H.P.

The length of the scale (and hence the likely time taken to complete and analyse it), made it a favourable choice over the S.I.P., since it was unrealistic to expect clients with acute low back pain (who were often still in considerable discomfort) to take $20-30$ minutes to complete a questionnaire about function, that was only one of a number of outcomes. In addition, since the Aberdeen Low Back Pain Scale was designed for clients with back pain in general practice, on examination it also was deemed to cover the main dimensions of function that would be pertinent to this client group, including sleeping, which is omitted in the SF36 instrument.

Therefore, on balance, the Aberdeen Low Back Pain Scale was the instrument of choice.

\subsubsection{Selection of pain measures}

The difficulties of measuring pain are discussed in section 4.10. No single scale exists that was considered capable of measuring the severity and nature of pain adequately in our study. Therefore it was decided that three different scales would be used.

Firstly the N.R.S. was chosen for use by the G.P. at the initial consultation with the client and in every home assessment by the researcher. The scale was administered verbally, was quick to use and gave a simple rating of clients' present pain. Despite this useful baseline measure, the scale is clearly crude and only addresses severity of pain. Nevertheless, it was deemed more sensitive than the S.D.S. which only has 3 or 4 points, as opposed to 11 . 
Secondly, clients completed visual analogue scales of pain and pain relief during home visits. These were quick and easy to score, giving an idea of clients' perceived severity of pain. The researcher was on hand if the client was unsure how to complete the scale.

In addition to measuring the severity of pain, it was also considered important to try and gain an understanding of the nature of clients' pain. Therefore, the Pain Rating Index of the McGill Pain Questionnaire was chosen, which had a stronger psychometric profile than any other pain outcome measure considered in this research. In practise, this instrument proved to be one clients either loved or loathed.

\subsubsection{Selection of anxiety measure}

Regarding the selection of an anxiety outcome measure, ideally the S.T.A.I. would have been the instrument of choice, owing to its strong psychometric profile (unlike the Anxiety Thermometer and the M.A.A.C.L.), and design for use with adults in the general population (unlike the Leeds Scales and M.A.A.C.L.).

In addition, the S.T.A.I. does not involve any physical components that might render it inappropriate with clients experiencing low back pain (unlike the H.A.D. as discussed in section 4.11.3).

However, what precluded the S.T.A.I. from being chosen in its full form was its length. To take between 10 and 20 minutes to complete this scale, which was only one of a number of outcome measures, was considered inappropriate. Therefore, a short form of the state scale was selected, which addressed this issue.

\subsubsection{Selecting outcome measures in context}

For the work in this thesis, the selection of outcome measures was made, acknowledging the limitations of these instruments. The decision was sometimes difficult, with many factors to consider.

It must be emphasized that this selection was deemed suitable for our study design, our time-frame and our client population. It is not a precedent for all studies of clients with acute low back pain. The message is clear: Each study must be considered individually when selecting potential outcome measures, weighing up their particular merits and disadvantages within the relevant context. 


\subsection{Limitations}

Perhaps the first consideration should be to question the viability of measuring the concept of health status using essentially quantitative, self-report means, that include the clients' own image of their health and ability, as this forms an integral part of self-report instruments. However such perceptions are subject to both conscious and unconscious bias, e.g. secondary gain, which may affect the accuracy of any measurements taken.

To complicate matters further, Chambers argues that in primary care, defining health in terms of diagnoses is problematic because often only symptoms, and the client's functional status are known, not the underlying pathophysiology ${ }^{(28)}$. Delitto confirms that the vast majority of people with low back pain are without a diagnosis based on pathology ${ }^{(13)}$. However, it must be questioned whether it is necessary to have a precise understanding of a client's pathophysiology before attempting to measure their symptoms, or whether a label of 'low back pain' would suffice? It is clear from chapter two that attempts at classification of low back pain based on diagnostic criteria are unreliable, but is it reasonable to assume that the symptoms exhibited by a client with pain caused by a zygapophyseal joint dysfunction are comparable to those exhibited by a client with underlying discogenic pathology? Whilst acknowledging the academic nature of this debate, a pragmatic solution accepting 'low back pain' en masse can be reached, which is not ideal, and hence limits the whole concept of measuring health status. However, if in future, classifications can be reliably made based on the pathophysiology of low back pain, then this issue will need revisiting.

Assuming that health status can be measured quantitatively in clients with low back pain, the quality of the article reporting the development of an outcome measure greatly affects the way it fares when using methods similar to the one in this chapter. For example the brief description of the development of the Oswestry Low Back Pain Disability Questionnaire $^{(49)}$, one of the earliest condition-specific functional measures, may result in underselling the attributes if this measure, and hence its performance, in this review. Many later measures have more detailed descriptions of their development, and hence fare better in the tables.

Keeping an open, 'objective' mind is essential when reviewing outcome measures. As Brooks ${ }^{(103)}$ observes: 'one cannot escape the impression ... that particular (groups of) 
researchers become advocates for their favoured method(s)', and such biases should be acknowledged and discussed as a possible limitation in methodology.

Despite these limitations, it is important to not consider the development of outcome measures in a vacuum, but to keep in perspective the clinical significance of any changes in scoring. O'Brien ${ }^{(\text {in } 29)}$ warns that 'In interpreting clinical studies we need reasonable knowledge of disease and how it is measured and being overly impressed by power analyses and statistical theory may obscure important clinical results'. Again it is a delicate balance developing an instrument which will stand up to rigorous scrutiny in a research arena and yet is clinically useful.

In the international arena, it is difficult, if not impossible to make definitive statements about cross-cultural equivalence of measures, because adapting and applying measures internationally has resulted in vastly differing methodologies ${ }^{(66)}$. In addition, some outcome measures, such as the SF36, undergo careful and extensive translation procedures, whereas others, are simply translated into another language linguistically, and used in research with the assumption that the essential properties of the original measure have been preserved ${ }^{(66)}$. Potential cultural biases among items and scales has seldom been assessed ${ }^{(66)}$, which limits the concept of international self-report outcome measures. Despite these caveats, many published outcome measures have been found valuable in research, although their widespread use by clinicians is still awaited.

\subsection{Discussion}

From the work in this chapter, it is clear that outcome measures need to be simple and practical, in addition to being psychometrically sound. This will assist in their adoption in the clinical world, which to date, has not occurred ${ }^{(3)}$. Nelson et al. state that the best new measurement tools have been developed for research use, not for clinical practise ${ }^{(3)}$, but surely, widespread use of the 'best' tools will also enhance the evaluation of clinical interventions. Dissemination of the findings of systematic reviews, such as the one described in this chapter, should encourage further debate both within the research and clinical arenas.

It is paramount that details about instrument development are clearly stated, outlining the assumptions, sources of error, bias and any limitations. This should help prevent 
measures being used inappropriately in research studies, often in populations for which they were not designed. Researchers and clinicians, searching for appropriate instruments, need to scrutinise the fine detail of each measure under consideration.

There needs to be more comparative work between different outcome measures, such as the work discussed in chapter six in this thesis. Important differences in scale characteristics may only be apparent when multiple scales are tested among the same group of patients ${ }^{(68)}$. Apart from strengthening the psychometric profile of outcome measures, such work will also help decide whether measures should be used alone or in combination with other instruments. During this period of consolidation it is expected that some outcome measures will strengthen in psychometric profile and gain wider acceptance, whereas others may be rejected. However this selection process is necessary, since it is not the quantity of outcome measures that is important, but their quality, coupled with their ability to withstand both academic scrutiny and practical use.

It is inappropriate to continue using insensitive outcome measures, because if they are used clinically to evaluate a treatment modality, the true effect of a treatment may not be detected. This could have a huge impact, with the net result being to undersell any benefits from that particularly intervention. As an example, Coxhead et al. reported that manipulation was not found to be significantly better than other treatments when a 3-point scale of change of symptoms was used as the outcome measure. However, when improvement was measured using a V.A.S., manipulation was shown to be significantly better than other treatments ${ }^{(50,104)}$. This illustrates the serious danger of erroneous findings arising from research using insensitive outcome measures and the ongoing need for vigilance by both researchers and clinicians.

Apart from the huge ethical responsibility and safety issues surrounding evaluating interventions, the practical difficulties of developing new outcome measures are immense and were addressed in section 4.5. Therefore perhaps it would be prudent to focus on adapting existing measures of satisfactory quality, and validating them where possible, rather than starting anew. For example Roland and Morris successfully used the Sickness Impact Profile to develop their Disability Questionnaire ${ }^{(50)}$.

Whatever new advances are made in the measurement literature, it is crucial that the information is readily available, without cost, to all who seek it. There is now a worrying 
trend of researchers seeking payment for using their instruments, which will seriously hamper progress in this field. It will have implications on research, but in particular in getting these outcome measures into the clinical arena.

Whilst considering cost, both researchers and clinicians will need to decide whether the information gained from an outcome measure justifies the time and expense of the required data collection and analysis. In the end, whatever direction the path of outcome measures research takes, it is imperative that the client still remains the focus of attention.

\subsection{Summary of chapter}

- Measuring low back pain is an ongoing challenge facing all who work in the field.

- Many measurement tools exist. In our quest for finding and developing accurate outcome measures, it is necessary to consider the '3Ps': Purpose; Practical issues; and Psychometric properties. The relative importance of each of these domains will be influenced by the nature of the task e.g. research, clinical practise etc.

- The outcome measures selected for the 'Back Home' study are not a precedent for outcome measures in all studies of clients with acute low back pain. Each study must be considered individually. 


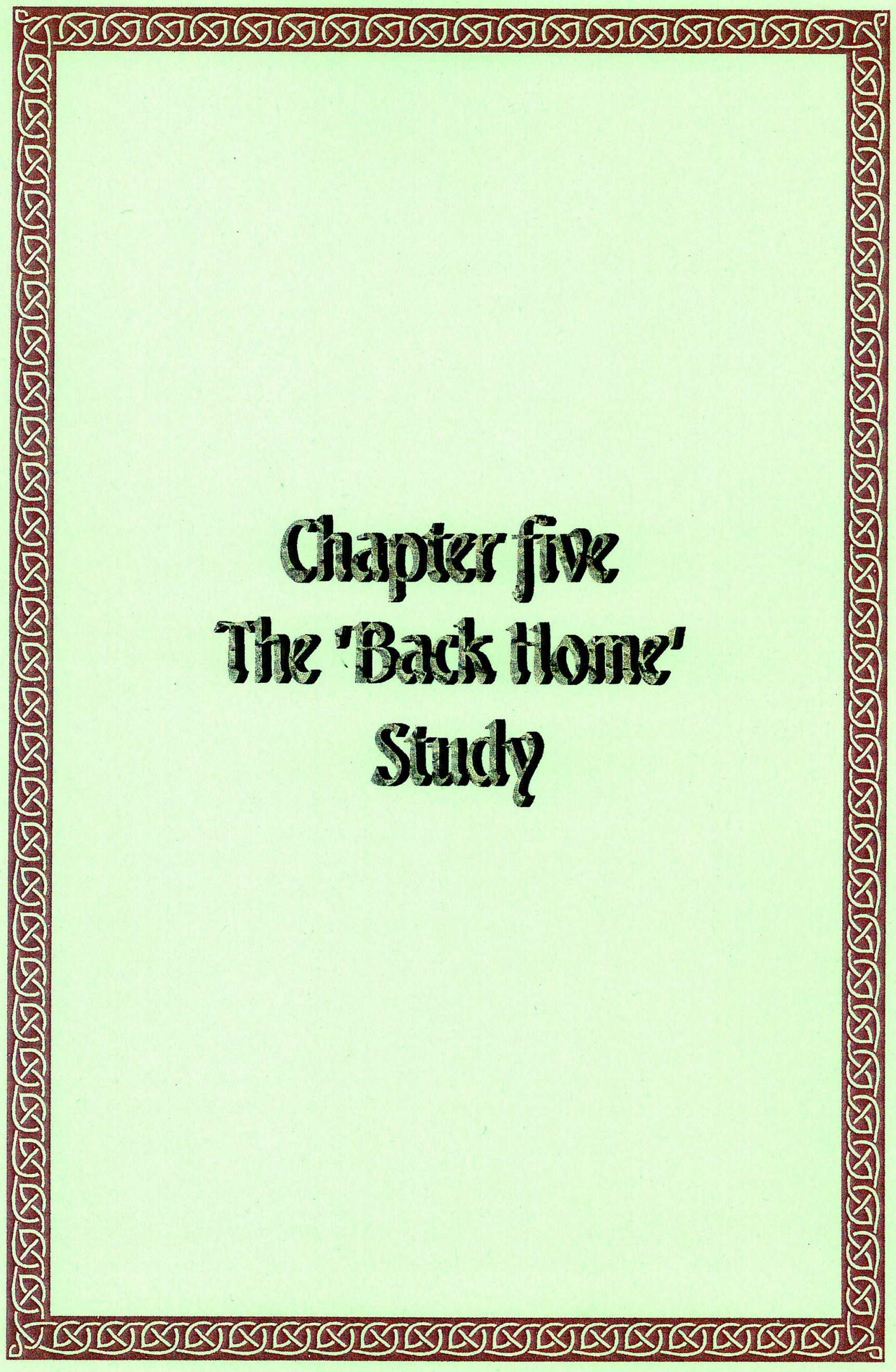




\section{Chapter five: The 'Back Home' Study}

From the G.P. questionnaire in chapter three, it is clear that managing low back pain challenges health professionals. Part of this challenge is enabling people to manage their symptoms at home, which includes them learning that their own self-care may have significant effects on their back pain.

This chapter addresses the key topic of health education, both in general, and specifically relating to client-information leaflets, since these underpin the research in this chapter. The research surrounds the development of a leaflet (called 'Back Home') and its subsequent evaluation in a randomized controlled trial of people experiencing acute low back pain. The leaflet was used to see whether it was possible to change clients' knowledge, attitude and behaviour in relation to their back pain, and then ultimately to see whether it was possible to influence their levels of function.

Such work has important implications for clinical practise, since promoting independence may encourage clients to take more responsibility for their health, help reduce repeated visits to health professionals, and thus reduce health care costs.

\subsection{Why educate clients in health issues?}

Many misconceptions surround client education. For example, Donovan et al. report that some health professionals '... tend to assume that education is a simple matter and that instruction of patients by doctors and other members of the team will be effective ${ }^{\text {(1) }}$. If only this were true!

Client education is a process assisting people to learn and incorporate health-related behaviours into everyday life: It is a process of helping people to change their behaviour $^{(2)}$. Perhaps it's worth considering whether clients actually want to be educated about health issues? This is certainly assumed in much of the health education literature, and this assumption also underpins the study described in this chapter. 


\subsubsection{Ethical considerations}

Before exploring health education more closely, there are some pertinent ethical issues to consider. Health professionals have '... ethical and legal obligations to inform patients about their disease and treatments so that they make informed decisions ${ }^{\text {(3). }}$.

It can be difficult to decide how much information to give to clients, as Redman says '... the amount [of information] that should be shared is largely considered to be a matter of professional judgement ${ }^{{ }^{(4)}}$. At one extreme the health professional does not want to overload the client, but at the other, according to Close, it is 'clearly negligent to assume that the client learns on their own'(5). This information is an essential component of the notion of informed consent, which forms part of the professional codes of practise for health professionals ${ }^{(6,7,8)}$.

However these codes of practise tend to assume that clients want to make informed decisions, which is not always true. For example, Beisecker found (in a study of information-seeking behaviours when rehabilitation medicine clients attended outpatient clinics in the U.S.A.), that although clients expressed a uniformly strong desire for medical information, they wanted to be knowledgeable about their medical care, but were much less willing to assume responsibility for medical decision-making. Instead they preferred to delegate that responsibility to doctors ${ }^{(9)}$. This can be a source of frustration for doctors when clients want to be given information about their medical conditions, yet are unwilling to use that information to make informed decisions regarding health care ${ }^{(9)}$.

Even if clients do want information in order to make informed decisions about their health care, it cannot be assumed that they will necessarily comply with the advice of health professionals - this is a different issue.

\subsubsection{Health education issues}

In simplifying this vast subject, the format chosen for presenting this information is based on Kipling ${ }^{(10)}$ who said:

'I keep six honest serving-men

(They taught me all I knew);

Their names are What and Why and When

And How and Where and Who.' 
What: Client education involves transferring knowledge from the health professional to the client. Therefore it must be perceived by the client as being relevant to their lay beliefs, expectations and current situation. It must be remembered that this transfer of knowledge can alter the balance of power and control in the relationship with the client ${ }^{(4)}$, and this is discussed further in chapter seven.

Why: Apart from the ethical reasons for promoting health, there is a high expectation for information among clients due to society's growing awareness of health and education ${ }^{(11)}$. Studies show that clients are often dissatisfied with the information they receive in hospital ${ }^{(5)}$, and paradoxically, those who complain about lack of information may be the best informed ${ }^{(11)}$. Other factors cited for this increase in client education include: legal pressures; promotion of self-care and preventive measures; earlier hospital discharge; increasing incidence of chronic disease; and a belief that client education improves compliance and hence health and well-being ${ }^{(12)}$.

There is clearly a demand for health education, although its effect, like any medical intervention, must be evaluated. Daltroy and Liang show that educational programmes do increase clients' knowledge ${ }^{(3)}$. There are many other claims: that improved communication leads to better treatment compliance and client satisfaction ${ }^{(11)}$; giving a client a role in their own recovery will reduce anxiety, enable the client to cope with the stress of illness better, reduce complications (e.g. post-operatively) and enhance recovery ${ }^{(5)}$ etc. In a meta-analysis which reviewed 102 client education studies in elective surgery, educational interventions were shown to reduce length of hospital stay, medical complications, length of time to return to normal activities and measures of pain as well as increasing self-reported well-being ${ }^{(13)}$.

In addition, there are resource-based arguments for empowering clients to become more actively involved in their own health care, aiming to '... free clinicians' time and skill to focus on the problems of the more severely ill patients ${ }^{{ }^{(14)}}$.

Specific to back pain, Cherkin argues that 'Because back pain is typically a recurrent problem that improves with time regardless of treatment, effectively teaching patients to take more responsibility for their own care remains a high priority ${ }^{(15)}$. Nordin claims that when clients with non-specific low back pain are given information, they are more satisfied at the first visit and require less health care ${ }^{(16)}$. She goes further in saying 'This 
points to the fact that it is the health care provider who needs to be educated, perhaps more than the patient' ${ }^{\prime(16)}$.

(Why not): It is worth considering that there may also be disadvantages of client education, which are often omitted in the literature. Tucker and Kirwan suggest possible adverse effects may include: increasing depression; heightened levels of helplessness and fear; confusion; and changes in lifestyle that may have detrimental effects on both personal and work relationships ${ }^{(17)}$. Such effects are probably more of an issue when educating clients about chronic diseases such as rheumatoid arthritis or cancer (due to the natural history and prognosis), than symptoms such as acute low back pain, nevertheless, they should not just be dismissed.

When: It cannot be assumed that clients want information at the first opportunity, or indeed are ready to receive it; they may be distraught and not ready to learn when information is first given ${ }^{(3)}$. An example of when education may be mis-timed can be seen in a study of a preoperative educational intervention with clients undergoing cardiac surgery, where it was suggested that the 'life-threatening nature of this type of surgery impedes learning and undermines the effectiveness of preoperative education in the immediate preoperative period ${ }^{\text {(18) }}$. Heightened levels of anxiety are (not surprisingly) thought to be a causative factor ${ }^{(18)}$. In the context of back pain, this is discussed further in section 5.10 .

How: There is a shift in emphasis from didactic programmes to methods of information provision that pay greater respect to clients' beliefs ${ }^{(1)}$. Clients do not just accept what they are told; it has to make sense and be justifiable within their way of looking at the world $^{(19)}$. Therefore it must be remembered that how clients are educated i.e. using different learning styles, is as important as the content of what is actually taught. Luker and Caress argue the '... applicability of mainstream educational principles to the teaching of patients who, by definition, are sick is questionable, and in many cases unworkable ${ }^{\text {(12) }}$.

There are also issues about sequencing the delivery of educational material, which may facilitate or hinder its impact. For example, evidence that the order of delivery of information is important, exists in a study of 'problem drinking', where people who were given strategies for self-regulation first and then education, had a significantly greater 
reduction of alcohol intake than a group who received these components in the reverse $\operatorname{order}^{(20)}$. Extrapolating this to back pain, as the pain perception changes, the approach to empowering and educating clients, needs to change also. Therefore we cannot expect our approach to suit clients at all stages in the natural history of low back pain.

Where: Following this argument, the consultation may not be the most effective place for client education ${ }^{(19)}$. Materials which reinforce this education and which can be taken away from the consultation, such as leaflets, books or videos, may help address this issue.

Who: Clients are coming to understand that knowledge about illness and medical care is not the exclusive property of health professionals ${ }^{(5)}$. Educating clients is a partnership, a two-way interaction, in which the agenda should be jointly set by both the client and the health professional. However, some clients may choose to assume a more passive role. The challenge for the health professional is in deciding how best to impart information to an individual client in a way which is most likely to be of benefit. In fact, Tucker and Kirwan question whether it is even possible to identify those clients most likely to benefit from education ${ }^{(17)}$. Unfortunately, the answer to this is complex and unclear at this time.

\subsubsection{Barriers to client education}

Health education programmes often err in believing they have influenced people to change health behaviour, and then report disappointing results ${ }^{(4)}$. Partly this may be because health beliefs are deep-rooted and resistant to change ${ }^{(16)}$, and partly because clients lack the skills to overcome habit or forces in their environment ${ }^{(3)}$, (assuming they are motivated to change).

Many factors have been suggested as barriers to client education ${ }^{(5)}$. On the part of the educator, these may include: lack of knowledge and/or confidence; poor communication and interpersonal skills; poor assessment skills which could render the teaching inappropriate; underestimates of clients knowledge and perceptions; lack of teaching skills; inability to engage clients in learning; lack of time; heavy workload; inadequate staffing; lack of desire; poor organization; and low priority given to client education. From the client's perspective, the general public does not always give health education a 
high priority and the client may not recognise the health professional as a source of information ${ }^{(5)}$.

Despite the diversity of these barriers, many can readily be overcome with adequate forethought and planning.

\subsubsection{Why use a leaflet?}

Having considered health education in general, it is worth looking at leaflets in particular, as this medium of client education underpins the study in this chapter.

Clients have complex sets of lay beliefs, drawn from their own experiences and that of their families ${ }^{(1)}$, which have been shown to lie behind much of people's behaviour concerning health and illness ${ }^{(19)}$, and also affect the way people cope with disease $\mathrm{e}^{(19)}$. Any advice that people are given (lay or medical) has to pass through this filter of lay beliefs ${ }^{(1)}$. Thus a leaflet can be used to help clients and health professionals identify some of these lay beliefs, to ensure that any health education and advice is relevant to the clients' needs.

One way of achieving this might be to move towards an interactive leaflet, inviting active participation by clients. For example clients with acute low back pain might be asked to think about activities or postures which help ease their symptoms. Here, the idea being that they have made the information relevant to their needs, which, combined with some ownership of this information, might empower them to use it to find antalgic postures when in pain. This is in keeping with Roux's principles of developing educational material: firstly the message must be simple; secondly, it needs to be relevant i.e. 'close to daily realities that patients have to deal with'; and thirdly, it is important to stimulate clients' observations of solutions to their own problems ${ }^{(21)}$.

Leaflets can be used to cover additional information and act as a reminder for a client following a medical consultation, since, according to Livesley and Rider, '... much that is said is forgotten'(22). Indeed, in a series of studies of clients with osteoarthrosis, those who were exposed to booklets had improved recall when compared to control subjects who were not exposed to the booklet ${ }^{(23)}$. However it must be remembered that the ability to recall does not necessarily mean that clients will either accept or make use of what they can recall ${ }^{(19)}$. 
It is also thought that giving information, such as a leaflet, can alleviate anxiety and promote psychological benefit ${ }^{(12)}$, and also reduce the medico-legal problems that occur with dissatisfied clients ${ }^{(22)}$. In addition, using a leaflet can enable people to absorb the content at a time and place which is appropriate to them, since many people may find it difficult to understand information that is given during a consultation.

Thus leaflets are portable and relatively inexpensive adjuncts to treatment. They may incorporate diagrams and pictures as other means of getting information to the reader. However, even this issue may be complex. In the series of studies investigating doctorpatient communication in clients with osteoarthrosis mentioned earlier, Moll studied five styles of illustration: cartoon, matchstick, representational, symbolic and photographic in educational booklets about osteoarthrosis ${ }^{(23)}$. Overall it was found that pictures in booklets do enhance communication ${ }^{(23)}$, but it depends on the presentation as to which style of illustration works best. Generally photographs emerged as the most generally preferred style although cartoons scored well when presented as booklet illustrations ${ }^{(23)}$. Moll claims that photographs 'fit with the thinking vogue of the era in which we live' ${ }^{(23)}$. They are usually immediately recognizable, relevant and people can identify with them. Meanwhile, cartoons are generally popular, may help assuage anxiety and have a 'warming' effect in promoting relaxation in a doctor-patient relationship ${ }^{(23)}$, although Moll does warn that cartoons may irritate clients if they are perceived as being 'inappropriate in a field that is far from humorous'(23).

It is not only the content and the presentation of leaflets that is important, but also the manner in which the leaflet is issued. Harland et al. argue that because doctors are viewed by clients as the primary source for information, leaflets may be best delivered by the doctors themselves during the consultation to achieve the most impact ${ }^{(11)}$. It is also stated that verbal reinforcement is essential, as some authors claim the written word always provokes some questions ${ }^{(22)}$. However, this concept is misleading as clients do not usually actually read the leaflet during the consultation, and therefore any questions that may arise as a result of the content, will occur at a later date, not at the time of any verbal reinforcement.

It is particularly important that the leaflet is not seen as a substitute for good communication between the doctor and client ${ }^{(11)}$. As Hadler says 'Does not everyone in 
pain deserve the empathy and human contact that can never occur in any "educational booklet?" (24). Mindful of this potential problem, it is also worth remembering that a complex and poorly presented leaflet is 'as bad as no leaflet ${ }^{(22)}$. Indeed it may be worse, as some leaflets exist with inaccurate content, as was discussed in section 3.6.8 and thus there is a need to ensure that educational material is regularly updated to ensure its content keeps abreast of changes in the underpinning knowledge base.

In relation to back pain, there is little reported evidence of the effects of client education in a population of people with acute low back pain. Roland and Dixon did report positive findings in a randomized controlled trial of an information booklet, where those who received the booklet reportedly saw their G.P. on fewer occasions in the ensuing year than the control group who did not receive the booklet ${ }^{(25)}$. Knowledge scores were also higher in the booklet group, but there were no differences reported between the two groups in terms of absence from work. Unfortunately the outcome measures did not included symptomatology or back protection behaviours, and despite the positive findings, care must be taken in interpreting the results as some methodological flaws were evident. For example the clients in the study had an unspecified duration of low back pain, and it is unclear whether the two groups were identical. In addition, clients were not truly 'randomized', as they were allocated into the control and experimental groups according to their date of birth.

\subsection{Aims of the 'Back Home' study}

Having considered the potential benefits and pitfalls of using leaflets, the first aim of this study was to develop a client-information leaflet, specifically designed for people with acute low back pain. The second aim was to test this leaflet to see whether it empowered clients in their own pain management, by:

- improving their knowledge of back pain management

- changing their back protection behaviour

- improving their symptoms, their level of function (e.g. by hastening their return to work) and thus reducing health costs. 
Thirdly the study aimed to produce written guidelines, not intended as a formal protocol, but more of an aide mémoire, which could assist G.Ps in their management approach to low back pain.

\subsection{Development of the 'Back Home' leaflet}

From the results of the G.P. questionnaire cited in chapter three, 58 G.Ps in Southampton and the New Forest reported giving back pain leaflets to their clients and they returned many examples with the questionnaires (as requested).

These leaflets were then reviewed together with others from: a) pharmaceutical companies; b) charities such as the National Back Pain Association, and Arthritis and Rheumatism Council; c) insurance companies; d) local hospital trusts; and e) those already in existence in the literature such as ('Back pain', produced by the University of Huddersfield Spinal Research Unit ${ }^{(26)}$ and The Back Book - your guide to back care ${ }^{(27)}$ ). The content of the leaflets was reviewed against advice given in current clinical practice (including the Backschools operating in Southampton University Hospitals Trust, where information is evidence-based whenever possible). In addition, any research evidence cited by the leaflet authors was established. The presentation style was also considered including leaflet and font sizes, use of diagrams and pictures, layout, readability and opportunities for interaction.

The 'Back Home' study aimed to develop a leaflet to offer self-help advice for people with acute low back pain. It was therefore necessary to undertake a needs analysis of clients who had recently experienced acute low back pain, to determine what specific information they felt should be included in the leaflet.

It was envisaged that this leaflet, once its development was complete, would be given by a health professional to people when they first sought medical advice, in order to help them manage their back problem at home. In the case of the 'Back Home' study, it would be the G.P. giving the leaflet to the client at their initial consultation.

The needs analysis and development of the leaflet took place in five stages, as discussed in sections 5.3.1- 5.3.5. 


\subsubsection{Stage 1}

A simple questionnaire was designed by the research team, to be given to clients on their first visit to their G.P. for an acute episode of low back pain. The questionnaire asked clients what issues they would like to discuss with their doctor about their pain and how to manage it. Overleaf there was a question containing 16 items identified by the research team, based on our clinical experience and concepts gleaned from the leaflets mentioned in 5.3, covering aspects of daily living or self-help strategies. Clients were asked to rate each of these items using a Likert scale with five categories: 'essential'; 'very important'; 'important'; 'not very important'; and 'not relevant'. (See appendix $5.1)$.

Having designed this simple questionnaire, 24 questionnaires were given to G.Ps in two practices in the New Forest (at Hythe Medical Centre). These practices were chosen partly because of convenience and partly because it was considered that they served people from a wide socio-economic background (from discussions with the G.Ps and 18 months experience working with the practice). When clients with acute low back pain attended any surgeries at these two practices, the G.P. asked them to complete the questionnaire, and return it to the researcher in the stamped addressed envelope provided.

During a four week period, seven questionnaires were returned by clients out of the 24 (response rate $=29 \%$ ). No attempts were made to follow up non-responders as participants were anonymous to the researcher. The results were collated, noting any categories identified in the open question which were not already in the list of 16 items. This revealed a further 11 items: what treatment is available; how the spine works; how to manage shopping; the easiest way to manage physical relationships; ways of getting fit; how soon the pain is likely to settle; preventing recurrence; causes of low back pain; advice on bedding and pillows; what makes up the spine; and getting back to social and leisure activities.

The results of the Likert scales where clients were asked to rate the 16 items were analysed by awarding a score of three points if a client deemed the item 'essential', two points if it was considered 'very important' and one point for the items deemed 'important'. No points were given if the item was deemed either 'not very important' or 'not relevant'. 
The total scores from the seven completed questionnaires were then calculated. The scoring system differentiated the 16 items into ten groups which were ranked in order of importance, and these results are presented in table 5.1.

\section{Table 5.1: Leaflet Development Stage 1}

Results from the leaflet development questionnaire scored on a Likert scale. Scoring: 3 = 'essential' items; 2 = 'very important'; 1 = 'important'; $0=$ 'not very important' or 'not relevant' items. $(n=7)$ The maximum score attainable for each item $=21(7 \times 3)$.

\begin{tabular}{|c|l|c|}
\hline Rank & Function & Score \\
\hline 1 & How to lift things & 16 \\
\hline 2 & How to sleep or rest comfortably & 14 \\
\hline 3 & $\begin{array}{l}\text { How to position myself (lying, sitting, standing) } \\
\text { Exercises }\end{array}$ & 13 \\
\hline 6 & How to do my daily tasks & 12 \\
\hline 7 & How to drive & 11 \\
\hline 8 & $\begin{array}{l}\text { Where to get help } \\
\text { How to control pain using self-help measures (e.g. hot water bottles) }\end{array}$ & 10 \\
\hline 10 & How to get about & 8 \\
\hline 11 & $\begin{array}{l}\text { The easiest way to get washed and dressed } \\
\text { Getting back to sports } \\
\text { How to cough and sneeze }\end{array}$ & 7 \\
\hline 16 & $\begin{array}{l}\text { Getting back to DIY } \\
\text { How to control my pain by using tablets }\end{array}$ \\
\hline 13 & 6 \\
\hline
\end{tabular}

The research team members were surprised that the item which the seven respondents considered most important was 'how to lift things'. With such minimal detail requested it is impossible to know what the respondents wanted to lift. It might be that they perceived themselves needing to lift young children for example, or a dependent relative or maybe lifting was a vital part of their work. The research team were expecting 'how 
to control my pain by using self-help measures' to rank more highly than equal $8^{\text {th }}$, but perhaps we should not have been surprised since those clients who are really interested in self-help may not choose to consult a health professional in the first place. Likewise the item 'how to do my daily tasks' was (surprisingly) rated only the $6^{\text {th }}$ most important item. A possible reason for this may be that this advice is thought more important by clients with severe pain than those with milder symptoms, but as information about the severity of the pain was not collected, no conclusions can be drawn about this issue. With hindsight, this information might have proved useful.

\subsubsection{Stage 2}

The results of this initial work were used to form a semi-structured interview schedule to gain more information about what clients would like to see in a leaflet designed for people with acute low back pain. A copy of the interview schedule can be seen in appendix 5.2. This method was chosen so that clients could respond verbally and the researcher could record these responses. Such a face-to-face interaction enabled clients to clarify or expand on any of their answers if necessary, which would not have been possible with a written questionnaire.

The population chosen for this stage was clients attending the Hythe and Southampton General Hospital out-patient physiotherapy departments with acute or subacute low back pain. Again, partly this decision was pragmatic but also it was considered important to seek opinions from clients attending different clinical settings - a small hospital and medical centre and a large teaching hospital, in case subtle differences existed.

Fifteen clients were approached and a total of 13 face-to-face interviews were completed. In addition, two telephone interviews were conducted (which were modified), where the clients were unable to attend an interview in person.

During the interview, clients were asked to rate 27 items of daily living, self-help measures or aspects of back pain, using the Likert scale already described. (These were the sixteen items from stage one plus the eleven extra items identified by the respondents in the open question.) In addition, clients were asked to select five of the 27 items that they considered to be the most important, and rank these five in order of (perceived) importance. 
Results were collated from the 13 interviews using a similar strategy to stage one and are presented in table 5.2.

The scoring system differentiated the 27 items into 15 groups and again 'how to lift things' ranked equal first, while 'how to control my pain by using self-help measures' fared only joint $9^{\text {th }}$, when ranked in order of perceived importance. Perhaps even more surprising for us, was 'how to do my daily tasks' which ranked equal $21^{\text {st }}$. We had clearly over-estimated the importance of this item with the population chosen, since within the research team, we had (anecdotally) predicted that this item was likely to be particularly important.

Having considered the total scores, the next analysis was of the 'top five' scores identified by the respondents. Here, the item they deemed most important for them scored five points, the second most important scored four points etc. As before the 13 scores were collated and the results are shown in table 5.3.

The 21 items selected by the 13 respondents were differentiated into 14 groups and interestingly, the top ten groups comprised single items. Now 'how the back works' had become the most important item (moving up from its position in $11^{\text {th }}$ place when the total scores were chosen). 'How to lift things' had moved down to $8^{\text {th }}$ place when only respondents top five choices were considered and 'how to control my pain by using selfhelp measures' was now the $5^{\text {th }}$ most important item. These results were very different from the previous two tables, presumably because of the more sensitive scoring system. 


\section{Table 5.2: Leaflet development stage 2. Total scores from interviews}

Results from stage 2 in the leaflet development questionnaire scored on a Likert scale: 3

- 'essential' items; 2 - 'very important'; 1 - 'important'; 0 - 'not very important' or 'not relevant' items. $(n=13)$ The maximum score attainable for each item $=39(13 \times 3)$.

\begin{tabular}{|c|c|c|}
\hline Rank & Function & Score \\
\hline 1 & $\begin{array}{l}\text { How to lift things } \\
\text { What treatment is available } \\
\text { Where to get help / further advice }\end{array}$ & 33 \\
\hline 4 & Getting back to work & 31 \\
\hline 5 & What exercises to do & 30 \\
\hline 6 & $\begin{array}{l}\text { How to position myself (lying, sitting, etc.) } \\
\text { What can I do to get / keep myself fit }\end{array}$ & 29 \\
\hline 8 & How soon is my back pain likely to settle & 28 \\
\hline 9 & $\begin{array}{l}\text { How to sleep or rest comfortably } \\
\text { How to control pain using self-help measures (e.g. hot water bottles) }\end{array}$ & 27 \\
\hline 11 & How the back works & 26 \\
\hline 12 & How to modify my life to prevent my back pain coming back & 25 \\
\hline 13 & $\begin{array}{l}\text { How to drive } \\
\text { What are some of the causes of low back pain }\end{array}$ & 24 \\
\hline 15 & How to modify my bedding and pillows to help my pain & 23 \\
\hline 16 & What makes up the spine & 22 \\
\hline 17 & $\begin{array}{l}\text { How to get washed or dressed easily } \\
\text { How to control my pain by using tablets } \\
\text { Getting back to social and leisure activities } \\
\text { Getting back to physical relationships }\end{array}$ & 21 \\
\hline 21 & $\begin{array}{l}\text { How to get about } \\
\text { How to do my daily tasks } \\
\text { How to cough and sneeze }\end{array}$ & 17 \\
\hline 24 & $\begin{array}{l}\text { How to manage the shopping } \\
\text { Getting back to sports } \\
\text { Getting back to gardening }\end{array}$ & 16 \\
\hline 27 & Getting back to DIY & 15 \\
\hline
\end{tabular}




\section{Table 5.3: Leaflet development stage 2. 'Top 5' scores from interviews}

Results from stage 2 in the leaflet development where interviewees selected their 'top 5 ' items and ranked them on a scale: 5 - 'most important' of these 5 items and 1 - 'least important'. $(n=13)$ The maximum score attainable for each item $=65(13 \times 5)$. Items that scored 0 are not listed.

\begin{tabular}{|c|c|c|}
\hline Rank & Function & Score \\
\hline 1 & How the back works & 28 \\
\hline 2 & How soon is my back pain likely to settle & 25 \\
\hline 3 & What exercises to do & 20 \\
\hline 4 & How to sleep or rest comfortably & 18 \\
\hline 5 & How to control pain using self-help measures (e.g. hot water bottles) & 17 \\
\hline 6 & Getting back to work & 12 \\
\hline 7 & What treatment is available & 11 \\
\hline 8 & How to lift things & 10 \\
\hline 9 & How to position myself (lying, sitting, etc.) & 9 \\
\hline 10 & What can I do to get / keep myself fit & 7 \\
\hline 11 & $\begin{array}{l}\text { How to do my daily tasks } \\
\text { What are some of the causes of low back pain } \\
\text { Getting back to physical relationships } \\
\text { Where to get help / further advice }\end{array}$ & 6 \\
\hline 15 & $\begin{array}{l}\text { How to control my pain by using tablets } \\
\text { How to modify my life to prevent my back pain coming back } \\
\text { What makes up the spine }\end{array}$ & 3 \\
\hline 18 & How to get washed or dressed easily & 2 \\
\hline 19 & $\begin{array}{l}\text { How to get about } \\
\text { How to drive } \\
\text { Getting back to social and leisure activities }\end{array}$ & 1 \\
\hline
\end{tabular}


With such a small sample of clients, it is difficult to draw conclusions about the generalisability of the sample. However, it was considered essential to look at the needs of people who had experienced back pain before and compare these with people who were experiencing their first ever episode of back pain. Therefore, a subgroup of clients was identified who were experiencing their first episode of acute low back pain $(n=5)$ and their results were analysed. It was anticipated the needs of this group would not be identical to clients presenting with recurrent low back pain (who may have learned from previous experience) and it was therefore necessary to ensure the leaflet was developed addressing the needs of both client groups. The results of the subgroup were analysed in the same way as the whole group - firstly as total scores (presented in table 5.4) and then clients' top five items (presented in table 5.5).

Results from the subgroup were similar to the total scores, with five out of the top six items in each list being identical. Again 'how to lift things' ranked jointly first and both 'how to control my pain by using self-help measures' and 'how to do my daily tasks' were well down the list of priorities (joint $10^{\text {th }}$ and $23^{\text {rd }}$ respectively). Overall, these results were not so well differentiated (since there were only ten categories), which is presumably due to the smaller sample size and possibly clients wanted to know more, as several items scored near the maximum 15 points.

When analysing the 'top 5' items from the subgroup of clients with experiencing their first episode of acute low back pain, nine categories emerged from the scoring. 'How the back works' was clearly seen as the top priority. Interestingly, 'how to do my daily tasks' and 'how to control my pain by using self-help measures' both scored more highly than 'how to lift things'. This suggests that lifting was more of an important consideration for people experiencing repeated episodes of acute back pain than those who were experiencing acute back pain for the first time. 


\section{Table 5.4: Leaflet development stage 2. First back pain episode (totals)}

Results from stage 2 in the leaflet development questionnaire scored on a Likert scale: 3 - 'essential' items; 2 - 'very important'; 1 - 'important'; 0 - 'not very important' or 'not relevant' items. $(n=5)$ The maximum score attainable for each item $=15(5 \times 3)$.

\begin{tabular}{|c|c|c|}
\hline Rank & Function & Score \\
\hline 1 & $\begin{array}{l}\text { How to lift things } \\
\text { What treatment is available }\end{array}$ & 14 \\
\hline 3 & $\begin{array}{l}\text { How the back works } \\
\text { How to position myself (lying, sitting, etc.) } \\
\text { What exercises to do } \\
\text { Where to get help / further advice }\end{array}$ & 13 \\
\hline 7 & $\begin{array}{l}\text { How to modify my life to prevent my back pain coming back } \\
\text { How to modify my bedding and pillows to help my pain } \\
\text { What can I do to get / keep myself fit }\end{array}$ & 12 \\
\hline 10 & $\begin{array}{l}\text { How to sleep or rest comfortably } \\
\text { How to control pain using self-help measures (e.g. hot water bottles) } \\
\text { What makes up the spine } \\
\text { Getting back to work }\end{array}$ & 11 \\
\hline 14 & $\begin{array}{l}\text { How to get about } \\
\text { How to drive } \\
\text { What are some of the causes of low back pain }\end{array}$ & 10 \\
\hline 17 & $\begin{array}{l}\text { How soon is my back pain likely to settle } \\
\text { How to control my pain by using tablets } \\
\text { How to cough and sneeze } \\
\text { Getting back to social and leisure activities }\end{array}$ & 9 \\
\hline 21 & $\begin{array}{l}\text { How to get washed or dressed easily } \\
\text { Getting back to physical relationships }\end{array}$ & 8 \\
\hline 23 & How to do my daily tasks & 7 \\
\hline 24 & $\begin{array}{l}\text { How to manage the shopping } \\
\text { Getting back to sports }\end{array}$ & 5 \\
\hline 26 & $\begin{array}{l}\text { Getting back to DIY } \\
\text { Getting back to gardening }\end{array}$ & 4 \\
\hline
\end{tabular}




\section{Table 5.4: Leaflet development stage 2. First back pain episode ('top 5')}

Results from stage 2 in the leaflet development where interviewees selected their 'top 5 ' items and ranked them on a scale: 5 - 'most important' of these 5 items and 1 - 'least important'. $(n=5)$ The maximum score attainable for each item $=25(5 \times 5)$. Items that scored 0 are not listed.

\begin{tabular}{|c|l|c|}
\hline \hline Rank & Function & Score \\
\hline 1 & How the back works & 15 \\
\hline 2 & What exercises to do & 11 \\
\hline 3 & How soon is my back pain likely to settle & 9 \\
\hline 4 & How to do my daily tasks & 6 \\
\hline 5 & $\begin{array}{l}\text { How to sleep or rest comfortably } \\
\text { What treatment is available }\end{array}$ & 5 \\
\hline 10 & $\begin{array}{l}\text { How to lift things } \\
\text { What are some of the causes of low back pain }\end{array}$ & 4 \\
\hline 12 & What makes up the spine & 2 \\
\hline 13 & $\begin{array}{l}\text { Wow to get about } \\
\text { Wetting back to social and leisure activities }\end{array}$ & 1 \\
\hline
\end{tabular}

\subsubsection{Stage 3}

To date, information had been sought from people who had recently experienced low back pain. Now, the Likert scale with 27 items was also given to clients attending a Backschool at Southampton General Hospital $(n=9)$. These clients had experienced low back pain for a number of weeks (due to waiting lists and duration of physiotherapy treatment), and they were deemed an appropriate population to consult as their symptoms were subacute and therefore they could reflect how important issues were over a period of a few weeks. At the end of a Backschool session, clients were asked to complete the 
form listing the 27 items, which had been part of the interview schedule in stage two. Their responses were collated and are presented in table 5.6.

Interestingly these results were not well differentiated, with 15 items in the top five categories. It appears that in this population (who had experienced their symptoms for longer than people in stages one and two) were becoming more passive - with 'what treatment is available' as top of their list, and 'how to control my pain by using self-help measures' only ranked $22^{\text {nd }}$. It would be interesting to pursue this issue further, to establish the reasons for the onset of this more passive role. Could it be that the sample size is too small, or is it due to the duration of symptoms, or perhaps it is induced by attending a hospital out-patient (physiotherapy) department for treatment? It is likely to be due to a combination of factors, but nevertheless, health professionals need to reflect on their approach to ensure we do not promote dependency of clients upon 'treatment', and to see whether we have maximally promoted the self-help aspect of acute low back pain alongside any treatment modalities we may use, otherwise clients may assume a passive role and become further dependent upon health services. 


\section{Table 5.6: Leaflet development stage 3: Results from Backschool clients}

Results from stage 2 in the leaflet development questionnaire scored on a Likert scale: 3

- 'essential' items; 2 - 'very important'; 1 - 'important'; 0 - 'not very important' or 'not relevant' items. $(\mathrm{n}=9)$ The maximum score attainable for each item $=27(9 \times 3)$.

\begin{tabular}{|c|c|c|}
\hline Rank & Function & Score \\
\hline 1 & What treatment is available & 23 \\
\hline 2 & $\begin{array}{l}\text { How soon is my back pain likely to settle } \\
\text { How to modify my life to prevent my back pain coming back } \\
\text { What are some of the causes of low back pain }\end{array}$ & 22 \\
\hline 5 & $\begin{array}{l}\text { How the back works } \\
\text { How to lift things } \\
\text { What exercises to do } \\
\text { Where to get help / further advice }\end{array}$ & 21 \\
\hline 9 & $\begin{array}{l}\text { How to sleep or rest comfortably } \\
\text { How to get about } \\
\text { What can I do to get / keep myself fit } \\
\text { What makes up the spine } \\
\text { Getting back to work }\end{array}$ & 18 \\
\hline 14 & $\begin{array}{l}\text { How to get washed or dressed easily } \\
\text { How to modify my bedding and pillows to help my pain }\end{array}$ & 17 \\
\hline 16 & $\begin{array}{l}\text { How to do my daily tasks } \\
\text { Getting back to social and leisure activities }\end{array}$ & 16 \\
\hline 18 & $\begin{array}{l}\text { How to position myself (lying, sitting, etc.) } \\
\text { Getting back to physical relationships }\end{array}$ & 15 \\
\hline 20 & $\begin{array}{l}\text { How to drive } \\
\text { How to manage the shopping }\end{array}$ & 14 \\
\hline 22 & How to control pain using self-help measures (e.g. hot water bottles) & 13 \\
\hline 23 & Getting back to gardening & 12 \\
\hline 24 & $\begin{array}{l}\text { How to cough and sneeze } \\
\text { Getting back to sports }\end{array}$ & 11 \\
\hline 26 & How to control my pain by using tablets & 10 \\
\hline 27 & Getting back to DIY & 9 \\
\hline
\end{tabular}




\subsubsection{Stage 4}

The findings from stages two and three were used to draft the 'Back Home' leaflet. Since it was anticipated that the leaflet would be given out by health professionals, it was important to also seek their opinions on the content of the leaflet. Therefore a discussion group was organized for 12 senior chartered physiotherapists working with musculoskeletal clients across the Southampton District.

There were notable differences between the type of information identified by people who had experienced acute low back pain and health care professionals. For example the physiotherapists were very keen to include details of simple anatomy to explain 'what makes up the spine': Clients who were interviewed in stage two ranked this $16^{\text {th }}$ (in table 5.2) overall, and jointly $15^{\text {th }}$ when considering the top five scores (in table 5.3), only scoring three points. For clients experiencing their first episode of acute low back pain, this topic did not feature at all in their ratings of the top five most important items.

It was interesting that people with back pain were more interested in the mechanics of how the back works, but less interested in its anatomy. This is an important message for the content of educational sessions such as Backschools.

Another area of considerable controversy was giving advice on specific back exercises. There were strong opinions expressed by some of the physiotherapists, advocating specific exercises e.g. extension, flexion, general mobility exercises, strengthening, and co-ordination. However, others strongly opposed advocating any specific exercises without actually assessing individual clients. Eventually after much debate, the consensus was to omit details of any specific exercises in the leaflet. Again this conflicted with the clients' agenda - in stage two overall 'what exercises to do' was ranked $5^{\text {th }}$ (in table 5.2), and $3^{\text {rd }}$ (in table 5.3), when the top five items were ranked in order of importance. For the subgroup of clients who were experiencing acute low back pain for the first time, they perceived this issue as even more important, coming jointly $3^{\text {rd }}$ overall (in table 5.4), and $2^{\text {nd }}$ (in table 5.5), when their top five items were identified.

\subsubsection{Stage 5}

Having established an agenda for the content of the 'Back Home' leaflet from those clients who might receive such a leaflet and those health professionals who might issue 
one, the final draft of the leaflet was developed (see appendix 5.3). Where possible needs of both groups were addressed, but areas of controversy still remained, such as the inclusion of exercises. Here the research team made the final decision.

The definitive 12 page 'Back Home' leaflet was DL size $\left({ }^{1} / 3 \mathrm{~A} 4\right)$, with green, monotone print on ivory paper. It had interactive aspects that empowered clients to identify positions that make their symptoms worse and those which eased their pain. It also covered spine design, positions to help ease pain, information about getting moving, general exercise and fitness, practical hints and useful addresses. The inside back cover was left blank to be developed into a pocket where inserts could subsequently be added covering additional relevant topics of interest if the leaflet proved to be effective, (e.g. specific exercises taught at the Backschool, further advice about gardening, using a visual display unit etc.).

In the final version of the leaflet, there were 17 simple pictures and the text was simplified to give key messages in the simplest manner. The readability of the leaflet was calculated to be 6.5 on average, using the Gunning-Fog Index, which is within the grades recommended for health education leaflets (grade 5-9) ${ }^{(28)}$.

This simple leaflet was then evaluated with clients attending Backschools at Southampton General Hospital and the Royal South Hants Hospitals $(n=10)$. Clients were asked to read the leaflet and complete an evaluation sheet (see appendix 5.4) based on the 'Tell us what you think' format devised by Krilyk and Eyles ${ }^{(29)}$. A summary of clients' comments is included in appendix 5.4.

Overall the difficulty of the words and sentences was unanimously considered to be "just right', the page size was generally considered 'just right' and the pictures were deemed helpful. The majority of clients found the print size 'too small'. Regarding content, the information was unanimously considered 'just right' to understand and the amount was thought to be about right. Although most clients found the leaflet 'very helpful', they all considered the information was given 'too late' (which is not surprising as the clients were from a Backschool, whereas the leaflet was designed to be given when someone first presents with acute back pain). Despite the favourable appearance of this evaluation, it must be remembered that the evaluation tool was crude, with only three choices for most answers. 
Clients specified two categories which they would have liked more information about, which were 'posture' and 'exercises to strengthen my back'. No categories were identified which clients thought could be omitted, and four general comments were made; three relating to the clarity of the diagrams (which could be improved), and one person had described the leaflet as a 'handy referral point'.

Final amendments were made to the clarity of the diagram and font size (where possible) and the leaflet was then printed.

\subsection{The diagnostic guide}

Section 3.8 describes the diagnostic guide developed by the 'Back Home' team for optional use by G.Ps participating in the study. This guide was developed as an aidemémoire to help G.Ps identify signs and symptoms during the client examination that may indicate that serious pathology is present. The 'Back Home' team identified that it would be difficult to monitor exactly how the diagnostic guide was to be used throughout the study, hence its introduction as an optional guide.

If G.Ps did identify a client with danger signs present, then they were advised to seek further advice immediately from medical colleagues on duty at Southampton General Hospital. This arrangement was made in collaboration with the orthopaedic, accident and emergency departments, Wessex Neurological Centre, and radiologists at Southampton University Hospitals Trust. (This advice was irrespective of whether the G.P. had used the diagnostic guide, or made the diagnosis by other means.)

\subsection{Hypothesis (and null hypothesis)}

The hypothesis for this study was that an optional G.P. diagnostic guide plus a clientinformation leaflet improves the ability of people with acute low back pain to manage the episode of pain at home.

Conversely, the null hypothesis was that an optional G.P. diagnostic guide plus a clientinformation leaflet makes no difference to the ability of people with acute low back pain to manage the episode of pain at home. 


\section{Figure 5.1: The "Back Home" Study}

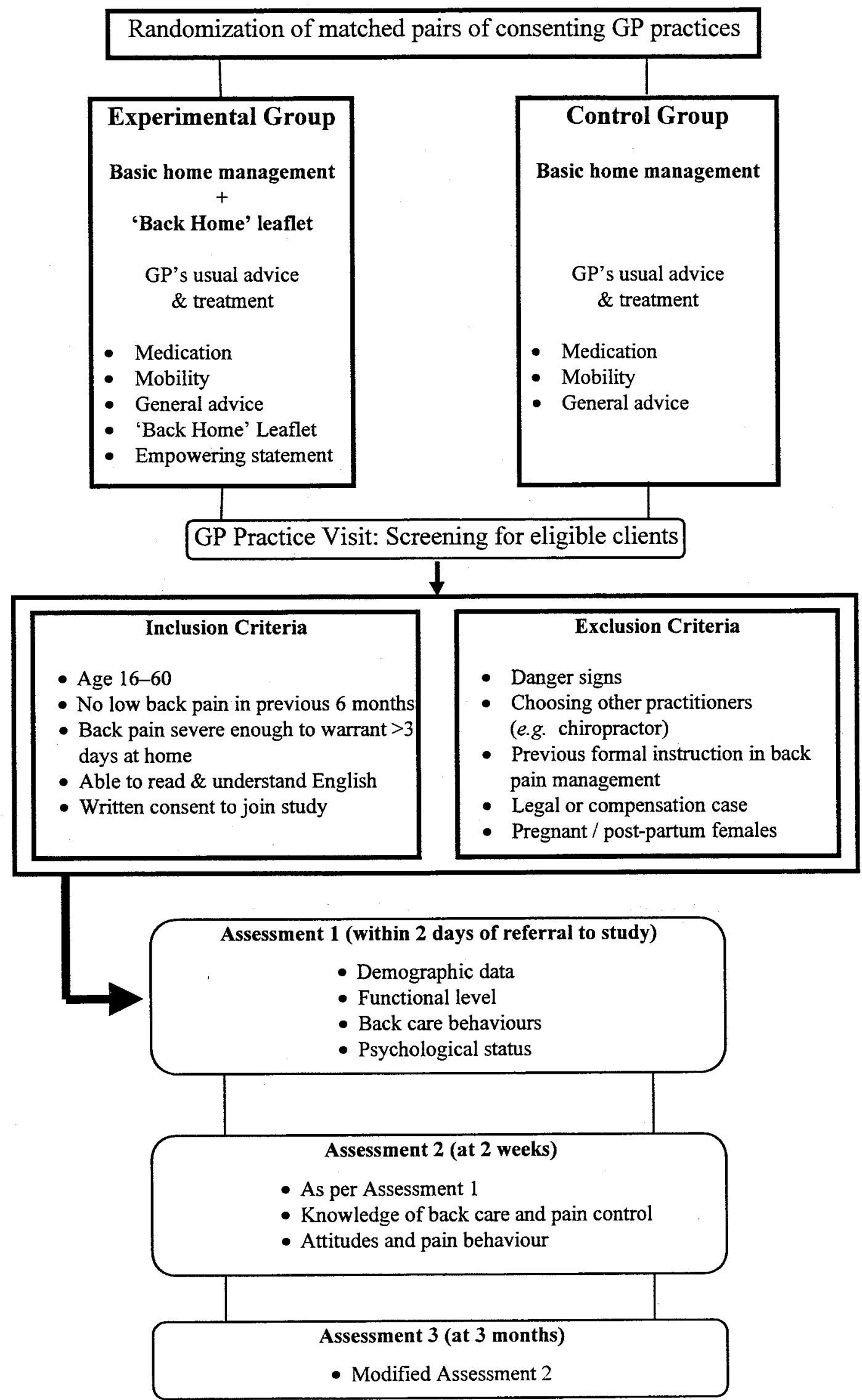




\subsection{Methods}

A summary of the methods is shown in figure 5.1.

\subsubsection{Study design}

The leaflet was tested in a blinded randomized controlled trial, which was undertaken in Southampton and the New Forest between June 1994 and December 1996. During this study, participating G.Ps had the opportunity to use the optional diagnostic guide.

\subsubsection{Ethical approval}

In 1993, ethical approval was granted from the Southampton and South West Hants Health Authority Ethics Committee (submission number 68/93) for this study.

\subsubsection{G.P. recruitment}

Having completed the questionnaire (described in chapter three), all G.Ps in the New Forest area $(n=97)$ were contacted by letter, outlining the randomized controlled trial to test the 'Back Home' leaflet, seeking their interest in participating. Positive contacts were followed-up by telephone call or practice visit (their choice), to discuss the study and explain their role more fully. In total 29 G.Ps in 16 practices consented to take part. (It was not a requirement that all G.Ps in a practice were involved - the decision lay with individual G.Ps.)

Each participating G.P. signed a consent form (appendix 5.5), agreeing to:

- strongly support the use of the leaflet if they were in the experimental group

- not use any other leaflets on back pain for the duration of the study

- not change the advice given to clients with acute low back pain for the duration of the study

- identify appropriate clients with acute low back pain and get their written consent to participate

- notify the researcher of changes in the client's treatment plan e.g. referrals elsewhere

- record any questions asked, if clients contacted the practice during the trial. 
In practise, due to the slow recruitment of clients into the study by G.Ps (discussed in chapter eight), the catchment area was increased to include a total of 51 G.Ps from 26 practices in Southampton and the New Forest.

\subsubsection{Randomization}

The G.P. practices were matched by location (in an attempt to control for obvious socioeconomic differences) and also by number of participating G.Ps in the practice (to help overcome any imbalance). The number of participating G.Ps in each practice ranged from one to nine.

Then, having established matched pairs of G.P. practices, randomization occurred within each pair using computer generated random numbers. The outcome was unknown to the researcher (who was therefore 'blind'), as another member of the research team completed the randomization and accompanying administration.

Participating G.Ps were then notified by letter whether they were in the control or experimental group and they were sent the packs of paperwork to start recruiting clients (see appendix 5.6).

Using such a strategy did assume that G.Ps would refer clients at similar rates into the study, which naturally did not occur. However, it was considered that using randomized matching pairs would attempt to get as close a matching between the control and experimental groups as possible.

Using this method, randomization occurred at the practice level, rather than at individual G.P. or client level. This decision is discussed in section 5.10.2, which compares the merits of randomizing at different levels.

\subsubsection{Inclusion / exclusion criteria}

As well as receiving the packs to start recruiting clients into the study, G.Ps also received a laminated A5 card of the inclusion criteria for the study (see appendix 5.7) to be displayed on their desk or notice board, as a physical reminder of the study. 
The inclusion criteria for entering clients into the 'Back Home' study were:-

- Age 16-60 years. These limits were set both to exclude paediatric clients (defined as $<16$ years) and to try to minimize the risk of symptoms secondary to osteoporosis, which increase with age.

- Taking at least three days off work (or equivalent). This was an attempt to exclude clients with mild symptoms by ensuring they were unable to work or function at home due to the severity of their pain. This criterion did potentially exclude self-employed clients who, for financial reasons, were unable to take time off. (Clients who were self-employed and had to take time off due to the severity of their symptoms were still included).

- Having experienced no back pain within the previous six months. This was to exclude clients with recurrent or chronic low back pain.

- Ability to read and understand English.

- Giving written consent to join the study (see appendix 5.8).

Exclusion criteria were:-

- Not fulfilling any of the inclusion criteria.

- Previous formal instruction in back pain management e.g. attending a Backschool or equivalent (as the intervention was educationally-based).

- Presence of danger signs: The optional diagnostic guide aimed to help G.PS to identify clients needing urgent hospital advice (who were therefore excluded from the study).

- The client was pursuing legal action or claiming for compensation.

- Choosing treatment from other practitioners, (e.g. physiotherapy, chiropractic or osteopathy), prior to the second assessment (at two weeks).

- Pregnant and post-partum female clients.

Clients who were inaccessible by telephone were not excluded from the study in an attempt to minimize socio-economic bias. 
Initially it was anticipated that the clients referred to the "Back Home" study would be experiencing their first episode of acute low back pain. However, pilot work (discussed in section 5.8) revealed that this was unrealistic and hence clients who had experienced low back pain previously (but not during the past 6 months), were eligible to participate. (In fact, in the actual study, only $25 \%$ of participating clients had never experienced acute low back pain previously.)

\subsubsection{The intervention}

In the control group, the G.P. continued with their normal management and advice for clients with acute low back pain. G.Ps in the experimental group in addition handed a copy of the 'Back Home' leaflet to the client, giving a standardized empowerment statement, to maximize any potential benefit of the leaflet. (See appendix 5.9).

It should be recognized that there was likely to be wide variation in the G.Ps' normal management of back pain and advice given, as this was impossible to standardize. Therefore, in this pragmatic trial, variations in practice were measured and analysed (see chapter 3). (It was possible to control in the analysis for baseline demographic between clients in the control and experimental groups, but it was not possible to control for differences in G.P. management in a similar way, as there were too many variables to control for.)

\subsubsection{Client recruitment and consent}

The G.P. (knowing whether they were in the control or experimental group) then approached appropriate clients explaining there was study under-way that was investigating the management of acute low back pain.

In order to establish that the control and experimental groups were comparable in terms of their acute low back pain, G.Ps were also asked to record, using a 0 - 10 numerical rating scale, the clients' perception of their pain. This could then be compared between the two groups, as an indication of the severity of back pain in both groups prior to any intervention.

Then, if the client had consented to participate, and was in the experimental group, the leaflet was mentioned and the empowerment statement was read. Recruitment in this way attempted to standardize the referral procedure and removed the potential bias of 'bribing' 
clients in the experimental group to take part with the leaflet. It was agreed that if the G.P. introduced the study stating there was an equal chance of receiving a leaflet as not, then this could adversely influence satisfaction outcomes from clients in the control group.

Once the client agreed to participate and the necessary paperwork was completed, the G.P. telephoned an answer-phone based at Southampton General Hospital to record the client's name, address and telephone number. This ensured that the first assessment could take place within two working days from the visit to the G.P.

\subsubsection{The sample size}

When the original application for funding was made, it had been estimated that 100 clients would be needed for the 'Back Home' study. This figure was based on the incidence of referral to physiotherapy for acute low back pain per annum, although the reasons for this are unclear. (At this time, it was not possible to complete a conventional sample size calculation as developmental work was needed on the outcome measures.)

\subsubsection{Assessments}

For the study, clients were followed up at their home by the researcher (a research physiotherapist) on three occasions: within two working days of the clients' visit to their G.P; at two weeks; at three months. The researcher was 'blind' as to whether clients were in the control or experimental group.

Assessment occurred via a battery of self-report questionnaires covering aspects of demography and psychosocial status. In addition, the researcher, (who, as a chartered physiotherapist, was experienced in the observation of movement) discreetly completed two very crude outcome measures about the client and their environment. Therefore, in total, 18 different outcome measures were used in this study, which are summarized in table 5.7, and these are discussed further in section 5.7.

During the client assessments, papers were completed in different orders to minimize any bias resulting from giving the outcome measures in a particular order. 


\subsubsection{Administration}

Following the second assessment, a letter was sent to the G.P. thanking them for referring the client into the study. This letter was for filing in the clients' medical records, in accordance with the Ethical Committee request.

Throughout the study an answer-phone was available (based in the physiotherapy department at Lymington Infirmary), as an advice line for any G.Ps in the experimental group to use if clients asked any questions arising from information in the leaflet, which the G.P. did not feel able to answer. This was never used.

The reason this separate answer-phone was installed was that if G.Ps did have any questions or issues arising from the leaflet, they could seek advice (from other physiotherapists) without revealing to the researcher that they were in the experimental group. It was a means of trying to ensure the researcher remained 'blind' throughout the duration of the study.

\subsection{Outcome measures}

Selecting the self-report instruments for measuring aspects of acute low back pain in the 'Back Home' study was complex, due to the diversity of dimensions measured and the plethora of instruments available.

Having reviewed the literature, networked with other researchers and clinicians and considered the opinions of members of the research team, it was decided to measure the following dimensions in clients with acute low back pain using self-report measures:

- demographic details (including details about treatment and management)

- control (locus of control and perceived control)

- knowledge

- attitude (towards their back pain)

- function

- pain

- anxiety

- satisfaction 
In addition, the researcher made discreet observations during the home visits, on aspects of the clients' behaviour and their home environment. To minimize potential bias, firstly the researcher was 'blind' as to whether the client had received a leaflet or not. In addition, the observation was designed to be a standardized checklist, with dichotomous responses, so that attempts to quantify aspects of behaviour were eliminated. (This instrument is discussed in section 5.7.4.) Nevertheless, it should be recognized that in any observation, there is still, the potential for bias.

Simple field-notes were also made, either during the home visit (if possible), or else in the car immediately afterwards, covering interesting comments made by clients, questions they asked and more detailed accounts of what they considered to be they aetiology and mechanisms of their back pain episode. It is recognized that the selection of material recorded and the omission of tape-recorded evidence renders these notes open to bias. However, initially these field notes were not intended to be used as part of the main analysis, but as means of recalling information about the client for subsequent visits and for verification of the data (see 7.5.2). Their importance became apparent during the analysis and interpretation of the findings. As an example, a number of these comments are used to illustrate points throughout this thesis and embellish the interpretation of findings.

The rationale for selecting instruments for measuring control, function, pain and anxiety has already been discussed in detail in chapter four, where many potential instruments were considered.

Collecting 'factual', demographic details about the participating clients and their treatment to date, was relatively straight-forward and the team set the agenda for the content, based on their clinical and research experience, and the reviews of back pain studies.

However, instruments for measuring attitudes, knowledge and satisfaction proved more controversial as either there were no readily available instruments which had been designed or validated for clients with back pain, or any instruments which did exist, were not deemed appropriate for our needs. Therefore further work was necessary to devise or revise these instruments. 


\section{Table 5.7: Outcome Measures Used in the 'Back Home' Study}

Table showing the outcome measures used in the 'Back Home' study.

* denotes the questionnaires that were developed by the research team as appropriate questionnaires were not available.

\begin{tabular}{|c|c|c|c|}
\hline Instrument & \multicolumn{3}{|c|}{ Assessment } \\
\hline Self-report & $\begin{array}{c}1 \\
\text { (2 days) }\end{array}$ & $\begin{array}{c}2 \\
\text { (2 weeks) }\end{array}$ & $\begin{array}{c}3 \\
\text { (3 months) }\end{array}$ \\
\hline Anxiety $^{(30)}$ & $\checkmark$ & $\checkmark$ & $\checkmark$ \\
\hline *Demographic details & $\checkmark$ & - & - \\
\hline *Attitude A & - & $\checkmark$ & - \\
\hline${ }^{*}$ Attitude B & - & $\checkmark$ & - \\
\hline *Back to work data & - & $\checkmark$ & $\checkmark$ \\
\hline Function $^{(31)}$ & $\checkmark$ & $\checkmark$ & $\checkmark$ \\
\hline Knowledge ${ }^{(\text {after 32) }}$ & - & $\checkmark$ & - \\
\hline *Management / treatment & - & $\checkmark$ & $\checkmark$ \\
\hline Multidimensional Health Locus of Control ${ }^{(33)}$ & $\checkmark$ & $\checkmark$ & $\checkmark$ \\
\hline Pain $\mathrm{I}^{\text {(after 34) }}$ & $\checkmark$ & - & - \\
\hline Pain II (after 34) & - & $\checkmark$ & $\checkmark$ \\
\hline Perceived control ${ }^{\text {(after 35) }}$ & $\checkmark$ & $\checkmark$ & $\checkmark$ \\
\hline *Satisfaction I & $\checkmark$ & - & - \\
\hline *Satisfaction II & - & $\checkmark$ & $\checkmark$ \\
\hline Pain diary ${ }^{\text {(after 34) }}$ & \multicolumn{2}{|c|}{$\begin{array}{l}\text { completed between } \\
\text { assessments } 1 \& 2\end{array}$} & - \\
\hline \multicolumn{4}{|l|}{ By researcher } \\
\hline *Home assessment I & $\checkmark$ & - & - \\
\hline${ }^{*}$ Home assessment II & - & $\checkmark$ & - \\
\hline *Observable behaviour & $\checkmark$ & $\checkmark$ & $\checkmark$ \\
\hline
\end{tabular}

\subsubsection{Knowledge measurement}

The most appropriate knowledge questionnaire (for our needs) in the literature was 'The Back Quiz', devised by Jessep ${ }^{(32)}$. This instrument, 'A crude multiple choice paper 
designed to indicate the patient's level of understanding of the information given during the course of treatment ${ }^{(32)}$, had originally been used as part of a pilot study comparing clients who attended a Backschool with those in an individual education control group. This tool was amended so that the final questionnaire consisted of nine multiple choice questions, seven of which were questions with a choice of five possible answers and a category for 'don't know'. In addition, there were two questions, each with a choice of five diagrams from which to select an answer, (see appendix 5.10). The answers to these questions could be found directly (or indirectly) in the 'Back Home' leaflet. It is important to remember, as suggested by Donovan et al., that multiple choice questions rarely assess whether clients understand information, nor whether they can make use of $\mathrm{it}^{(1)}$, and in this case, the modified Back Quiz was just assessing retention of information, not its application.

\subsubsection{Attitude measurement}

In order to investigate this aspect, it was necessary to look at clients' attitudes towards their acute back pain, for example what they considered the causative factors of their episode to be, and whether (or not) they perceived they could manage the symptoms on their own. Attitudinal objectives have recently attracted more attention and are coming to be regarded by some as important variables in the outcome of disease ${ }^{(17)}$.

Theoretically it was possible that clients who attributed the cause of their back pain as being beyond their own actions or behaviour (i.e. 'caused' by other people, bad luck etc.), may have perceived themselves as less able to manage the symptoms on their own, than those who thought their own behaviour had contributed to their pain. Hence causation may have some bearing on attitude measurement (and this is considered in section 7.5).

In the quest for instruments to try and measure attitudes, no instrument was found which could be used directly or modified (as happened for knowledge and satisfaction outcome measures). Therefore the research team devised questions that they perceived as relevant to clients' attitudes, which were not already covered in any of the other instruments, and these were tested and modified in the client pilot study. Again, this was not ideal practise, (but rather, a pragmatic solution) as it would be preferable to seek opinions from clients. The final format, which consisted of two parts can be seen in appendix 5.11. 
Initially clients were asked an open question 'What do you think caused your back pain? Once they had completed this without any prompts, they were given the second part which listed 18 possible contributory factors, and clients were asked to tick any that they felt may have caused their symptoms. There was one additional category called 'other (please specify)', in case additional factors were deemed pertinent by the client. Overleaf, there were four questions that sought clients' perceptions about managing their symptoms and preventing recurrences.

\subsubsection{Satisfaction measurement}

Regarding satisfaction, it was decided that just collecting information about clients' satisfaction with their overall treatment was insufficient. We wanted to know how satisfied (or otherwise) clients were with the amount and content of any information they had received (either directly from their G.P., or indirectly through the 'Back Home' leaflet). Not surprisingly, a satisfaction scale could not be found which addressed these issues, and so visual analogue scales (already discussed in chapter four) were modified and used.

The final instrument consisted of three visual analogue scales asking clients to mark the $(10 \mathrm{~cm})$ horizontal line to show their level of satisfaction with: the amount of information they have received for their back pain; the content of this information; and their overall satisfaction with the way their back pain was being managed. End markers were 'totally dissatisfied' and 'totally satisfied' (see appendix 5.12).

\subsubsection{Behaviour measurement}

Finally an instrument was needed to measure behaviour. Nothing could be found which was suitable and so a very crude observational checklist was devised (see appendix 5.13). This instrument, designed by the research team, consisted of a simple dichotomous observation of the client undertaking four activities (if appropriate):

i. if they were in bed, then observation of whether they got up through side lying

ii. if they were sitting, observation to establish whether they made any attempt to support their lumbar lordosis (e.g. with a pillow, cushion etc.) 
iii. how they got up from the sitting position (did they make any attempt to move to the front of the chair first?)

iv. lifting / picking a light object up from the floor (firstly did they attempt to bend their knees and secondly, did they have a suitably wide base of support [defined as having their feet greater than or equal to the distance of their shoulders]?).

The activities of lifting, resting and positioning had scored highly in the needs analysis for developing the leaflet (especially in stage one [table 5.1] and clients experiencing their first episode of low back pain [table 5.4]). This partially influenced our choice of activity, but the main reason these specific activities were chosen was because they were functional, and could be readily (and discreetly) observed: It was likely that clients would be sitting to complete the questionnaires.

Incidentally, throughout many visits, clients naturally picked something up from the floor, such as a pen, coffee mug, toys, jumper etc. However, if they did not, then the researcher surreptitiously left papers on the floor at the end of the visit. Whilst the ethics of this must be questionable, it gave rise to an opportunity for activity four to be undertaken, but if the client was experiencing anything more than mild pain, this was not done. Had any attempt been made to ask clients to pick something up from the floor, this would be invalid as it would probably cue them to do the task in a way that was different from their normal. (Even trying to observe them discreetly may have influenced their performance, just by the presence of the researcher.)

It was decided that a simple dichotomous scale would be used when observing behaviour, as any more sophisticated scale of grading these activities was likely to be unreliable, as no other checks for accuracy could easily be made, and a crude, simple tool was considered preferable to a more refined, but inaccurate one.

Having now got our collection of outcome measures, the next stage was to carry out a pilot test to establish some of their practical and psychometric properties.

\subsection{Client pilot study}

This pilot work was undertaken prior to starting the main study. 


\subsubsection{Aims of the pilot study}

The aim of the pilot study was to determine the psychometric properties of the instruments which had been adapted for the main study, and also to learn how easily clients could complete the questionnaires and also the time they took to do so. This information would assist in planning for data collection in the main study.

\subsubsection{The client population}

For this pilot, clients were approached who had been referred to physiotherapy for an episode of low back pain, and were currently on the waiting list at either the Royal South Hants or Southampton General Hospitals. Twenty clients consented to participate.

\subsubsection{Methods}

The twenty clients were asked to attend an appointment, where they completed outcome measures for attitude, knowledge, and satisfaction, in addition to the demographic details. It was also decided that the pain rating index of the McGill Pain Questionnaire ${ }^{(34)}$ and the perceived control measure (modified from Bradley et al.'s work with clients who had been diagnosed with diabetes) $)^{(35)}$ would also be included in the pilot study. In an attempt to validate the perceived control instrument, the Multidimensional Health Locus of Control ${ }^{(33)}$ instrument was also completed, although with hindsight, using these two measures for different aspects of control in this way was erroneous, and this is discussed further in chapter seven.

Clients were asked to complete the outcome measures on two separate occasions, seven days apart (within an appropriate time scale as suggested by Streiner and Norman, (who advocate that test-retest reliability measures should be taken $2-14$ days apart) ${ }^{(36)}$.

\subsubsection{Results of the client pilot study}

In the sample of twenty clients, six were male. The age of the whole group ranged from 23 to 68 years, with a mean of 42 years. These clients took on average 22 minutes to complete the six questionnaires (range $12-50$ minutes). 
Fifteen clients completed the second set of questionnaires, plus two who returned them by post as they were unable to return to the department. Reliability coefficients were calculated for the continuous variables ${ }^{\dagger}$, and these varied between $53 \%$ and $92 \%$.

\subsection{Findings from the 'Back Home' study}

Throughout this study, poor client recruitment by G.Ps was an issue despite numerous postal requests, telephone reminders and practice visits. Between June 1994 and December 1996 only 64 clients were successfully entered into the study from a total of 51 G.Ps, although one client dropped out of the study for personal reasons prior to the second assessment.

Such consistently slow recruitment resulted in further follow-up work with G.Ps, exploring this issue further since it had major implications for future projects with similar methodologies. This work is discussed in chapter eight.

For the 'Back Home' study, the primary outcome was assessment two, which was measured at two weeks. With such a large data set, in order to avoid the risk of 'data dredging', four primary outcome measures, knowledge, attitude, observable behaviour and function, had been specified in advance and the results of these are shown in tables $5.9-5.12$.

Overall the knowledge questionnaire, which asked about the cause of back pain, positioning, activities of daily living, exercise and applying ice / heat, was not significant, but the questions which specifically related to sitting posture and asking clients the best position to put on socks or tights were significant at the 0.05 level, as shown in table 5.9.

\footnotetext{
Thanks are due to Dr R Pickering for transposing the Epi-info data and calculating these coefficients.
} 


\section{Table 5.9: Knowledge scores}

$\overline{\text { Results from the knowledge questionnaire (9 items). The figures indicate the number of }}$ correct responses from 63 clients. ${ }^{*}=$ statistically significant at the 0.05 level.

\begin{tabular}{||l|l|c|c|c||}
\hline \hline 1 & Commonest cause of back pain & $\begin{array}{c}\text { Experimental } \\
\text { Group (n= 35) }\end{array}$ & $\begin{array}{c}\text { Control } \\
\text { Group (n=28) }\end{array}$ & $\begin{array}{c}\chi^{2} \\
\text { P value }\end{array}$ \\
\hline 2 & Least stressful position & $11(34 \%)$ & $4(15 \%)$ & 0.247 \\
\hline 3 & Most likely to worsen pain & $18(58 \%)$ & $15(58 \%)$ & 0.977 \\
\hline 4 & $\begin{array}{l}\text { Easiest position to put on } \\
\text { socks/tights }\end{array}$ & $13(41 \%)$ & $4(15 \%)$ & ${ }^{*} 0.036$ \\
\hline 5 & Application time for ice pack & $4(13 \%)$ & $1(4 \%)$ & 0.243 \\
\hline 6 & Frequency of position changing & $14(44 \%)$ & $9(35 \%)$ & 0.479 \\
\hline 7 & Tissue affected by heat pack & $20(63 \%)$ & $19(73 \%)$ & 0.393 \\
\hline 8 & Best sitting position & $19(59 \%)$ & $6(23 \%)$ & $* 0.006$ \\
\hline 9 & Best form of exercise & $31(97 \%)$ & $22(85 \%)$ & 0.098 \\
\hline
\end{tabular}

The analysis of the open attitude questionnaire (asking clients to list any factors they thought may have caused to their back pain) is discussed in chapter six, where the effects of attitude on control is considered. Therefore, only the most salient aspect of the second attitude questionnaire (which was not significant) is presented in table 5.10:

\section{Table 5.10: Attitude scores}

Results from the attitude question: 'Do you think you can manage your back pain on your own?' $(\mathrm{n}=63)$

\begin{tabular}{|c|c|c|}
\hline \hline & Experimental Group $(\mathbf{n}=\mathbf{3 5})$ & Control Group $(\mathbf{n}=\mathbf{2 8})$ \\
\hline Yes & $15(47 \%)$ & $14(54 \%)$ \\
\hline
\end{tabular}

Regarding observable behaviour, only one client was actually in bed at the time the second assessment was undertaken, and therefore the first activity was not included in 
the analysis as it was not appropriate to ask clients to lay down and then observe them getting up.

The results from observing clients sitting, getting up out of a chair and picking a light object up from the floor are presented in table 5.11. It can be seen that both sitting with the lumbar lordosis supported and maintaining a wide base of support during lifting, were statistically significant at the 0.05 level.

\section{Table 5.11: Observable behaviour}

Results from the observable behaviour questionnaire (total 5 items but one was not applicable in the majority of clients and therefore omitted from this table). * = statistically significant at the 0.05 level.

\begin{tabular}{|l|c|c|c|}
\hline & $\begin{array}{c}\text { Experimental } \\
\text { Group (n=35) }\end{array}$ & $\begin{array}{c}\text { Control } \\
\text { Group (n=28) }\end{array}$ & $\begin{array}{c}\chi^{2} \\
\text { P value }\end{array}$ \\
\hline Use of lumbar support & $13(41 \%)$ & $3(12 \%)$ & $* 0.014$ \\
\hline $\begin{array}{l}\text { Move to front of chair } \\
\text { before getting up }\end{array}$ & $27(84 \%)$ & $21(81 \%)$ & 0.718 \\
\hline $\begin{array}{l}\text { Picking up object: bend } \\
\text { knees }\end{array}$ & $30(94 \%)$ & $23(92 \%)$ & 0.797 \\
\hline $\begin{array}{l}\text { Picking up object: wide } \\
\text { base of support }\end{array}$ & $22(69 \%)$ & $3(12 \%)$ & $*_{0.000}$ \\
\hline
\end{tabular}

The results from the functional outcome measure (the Aberdeen Low Back Pain Scale $\left.{ }^{(31)}\right)$, which was discussed in chapter four, were summated and presented in table 5.12. The results are expressed as percentages (since if clients deemed question 17 about their sexual activity as either not relevant, or they chose not to complete the question, then the maximum total score was reduced by three points, and hence the denominator was not consistent throughout). It can be seen that there were no statistically significant differences between the control and experimental groups in their functional outcome, using this measurement scale. 


\section{Table 5.12: Function scores}

Results from the functional outcome measure: The Aberdeen Low Back Pain Scale $(n=63)$

\begin{tabular}{|l|c|c|c|}
\hline & $\begin{array}{c}\text { Experimental group } \\
(\mathbf{n}=\mathbf{3 5})\end{array}$ & $\begin{array}{c}\text { Control group } \\
(\mathbf{n = 2 8})\end{array}$ & P value \\
\hline Score on Aberdeen test & $38 \%( \pm 15)$ & $36 \%( \pm 16)$ & 0.616 \\
\hline
\end{tabular}

Having considered the results of the primary outcome measures, it was important to analyse the demographic details of the clients. These are presented in table 5.13:

\section{Table 5.13 Demographic Details of Participating Clients}

Demographic details of clients in the control and experimental groups of the 'Back Home' study. $(n=63)$

\begin{tabular}{|c|c|c|}
\hline & $\begin{array}{l}\text { Experimental } \\
\text { Group }(n=35)\end{array}$ & $\begin{array}{l}\text { Control } \\
\text { Group }(n=28)\end{array}$ \\
\hline Sex (male) & $22 \quad(63 \%)$ & $19(68 \%)$ \\
\hline Mean age (range) & 40 years $(17-59)$ & 40 years $(21-57)$ \\
\hline $\begin{array}{ll}\text { Social class: } & \text { I } \\
& \text { II } \\
& \text { III } \\
& \text { IV } \\
& \text { V } \\
\text { (missing) } & \\
\end{array}$ & $\begin{array}{rr}0 & (0 \%) \\
3 & (9 \%) \\
12 & (34 \%) \\
16 & (46 \%) \\
4 & (11 \%)\end{array}$ & $\begin{array}{|rr|}3 & (11 \%) \\
4 & (15 \%) \\
13 & (48 \%) \\
4 & (15 \%) \\
2 & (7 \%) \\
& \\
(2) & (7 \%) \\
\end{array}$ \\
\hline Manual work & $24 \quad(69 \%)$ & $11 \quad(41 \%)$ \\
\hline Paid employment & $28 \quad(80 \%)$ & $24 \quad(86 \%)$ \\
\hline $\begin{array}{l}\text { Highest educational } \\
\text { achievement (O-level/ } \\
\text { CSE/GCSE or lower) }\end{array}$ & $32 \quad(92 \%)$ & $15 \quad(54 \%)$ \\
\hline Current or previous smoker & $29 \quad(83 \%)$ & $19 \quad(67 \%)$ \\
\hline Regular exercise & $8 \quad(23 \%)$ & $12 \quad(43 \%)$ \\
\hline $\begin{array}{l}\text { Previous episodes of back } \\
\text { pain }\end{array}$ & $31 \quad(89 \%)$ & $16 \quad(57 \%)$ \\
\hline Previously consulted G.P. & $27 \quad(87 \%)$ & $11 \quad(69 \%)$ \\
\hline
\end{tabular}


Despite the randomization process, it can be seen that (by chance) there are some baseline differences between the demographic details of the two groups. These differences were identified as being significant at the $90 \%$ level using either MannWhitney tests (for social class and age variables), or chi-squared tests for the binary variables. The five significant baseline differences were:

- socio-economic class,

- whether occupation involves manual work,

- highest educational level attained,

- taking regular exercise,

- and previous episodes of back pain.

A subsequent analysis was undertaken controlling for these factors using logistic regression ${ }^{\dagger}$. After this further analysis, three of the four results presented in tables 5.9 and 5.11 (which were statistically significant in the first analysis) still remained significant.

The levels of significance were:

i) knowledge question 8 (best sitting position) $\mathrm{P}=0.004$

ii) observable behaviour - use of lumbar support whilst sitting $\mathrm{P}=0.003$

iii) observable behaviour - using a wide base of support when bending $P=0.000$

What was particularly interesting was that this difference in knowledge about best sitting position was then reflected in a change of behaviour, which the client was unaware they were being observed on.

\subsection{Discussion}

This section focuses on the study design, sources of bias, limitations and methodological issues about the leaflet content.

\footnotetext{
† Thanks to Dr R Pickering for performing this logistic regression.
} 
In section 5.1, it was shown that leaflets could be useful adjuncts to management regimes, helping to reinforce key messages. However, there is much debate about how these messages should be disseminated. For example Roux advocates avoiding giving solutions that are either 'right or wrong' claiming that this avoids a scenario where 'patients would feel insecure about the way to deal with their low back pains' ${ }^{\text {(21). This }}$ work informed the development of an educational book about back pain, although Roux has not reported an evaluation of the effectiveness of this book.

Surely avoiding giving possible solutions conflicts with evidence-based practise because if evidence exists to support a particular piece of advice about doing a task, then it needs to be presented as the recommended way to do that task? For example, Nachemson's work on lumbar disc pressures (discussed in section 3.6) has shown what happens to the intra-discal pressures in the lumbar spine in certain postures ${ }^{(37)}$. From this work, it is clear that the intra-discal pressure is greater in a posture involving sitting and flexing, than when lying supine. Therefore based on this evidence, clients can be reliably advised that they will probably find it easier to put their socks or stockings on when they are lying down rather than sitting and flexing, but this conflicts with Roux's approach.

As well as considering the content of information given, particular thought needs to be given to how information is conveyed to clients with acute low back pain. It was interesting to note that when clients in the 'Back Home' study were in severe pain, their ability to reason often seemed impaired - they appeared to function on 'survival skills'. It was only when their pain became more manageable, that they were able to function at a higher level. It could be the pain mechanisms that affect concentration and reasoning abilities, but also the medication that clients are taking is likely to be a contributing factor to their impaired cognition. Therefore perhaps Roux's suggestion of avoiding giving solutions that are either 'right or wrong ${ }^{(21)}$, might be more appropriate when empowering clients in chronic pain than those with severe, acute symptoms.

To illustrate this, one client (with pain levels of five out of ten on a numerical rating scale on the first assessment) reported that she was initially unable to do her part-time sedentary job (working as a typist), due to her symptoms. She worked flexible hours and was able to select her hours for being at work. Within ten days, she had tried to return to work (in the mornings as she always used to do), but was finding the pain still 
intolerable. During assessment two (at two weeks), when reviewing her pain diary, it was clear that her pain was always worst in the mornings and then eased considerably in the afternoons. When asked why she chose to work in the mornings when her pain was at its worst, she replied:

'It's never crossed my mind to go to work at a different time. I can't believe I didn't think of something so obvious'.

At assessment three (at three months), again she brought the subject up, still amazed at her 'Lack of common-sense'.

Another client commented:

'When I am in severe pain, I just want to know what'll get rid of it - I'm not interested in where the pain is coming from ... I just can't take that stuff in'. He later commented: 'It's as if someone has opened my head and stuffed it full of cotton wool.

Having considered issues about how clients adopt advice given in a leaflet, it is important to evaluate the performance of the leaflet itself. In the 'Back Home study, at two weeks there was a significant difference in clients' knowledge and behaviour in relation to sitting, when the experimental group (who had received the leaflet) were compared with the control group. The fact that an educational intervention may be able to change knowledge and behaviour regarding sitting contrasts with others, such as $\operatorname{Moser}^{(38)}$, who undertook a single blind, randomized controlled trial to investigate changes in knowledge and behaviour (self-perceived and observed via videotape), following attendance at a Backschool. Moser concluded from the study that the Backschool leads to a change in the way people believe they use their backs, but does not effect a behavioural change in people with low back pain ${ }^{(38)}$. However, although these findings are interesting, the two studies are not directly comparable due to the different educational interventions.

One of the key findings from research is that education is more successful when clients are taught how to incorporate learned health behaviour into daily routines ${ }^{(2)}$. Perhaps the reason the 'Back Home' study failed to show any change in function throughout, was that the leaflet needed to be more strongly relevant to clients' lifestyles. This could either be achieved through the health professional taking time to personalize the health 
education messages at the initial consultation (which may be unrealistic, considering G.P. surgery intervals), or by increasing the interactive content of the leaflet.

However, care must be exercised, as Smith questions whether there is a point at which too much information is provided, and there is a danger of sensory overload ${ }^{(2)}$. Again this point (if it does exist) in clients with back pain, will probably not be fixed, but is likely to vary throughout the natural history of resolution, so that there would be more likelihood of sensory overload in the early stages, when clients are less able to retain the information.

In evaluating the 'Back Home' leaflet, the significant difference in observable behaviour between those who received the leaflet and those clients who did not, is still reflected in assessment three (completed at three months), but was not sustained in functional outcome measures such as the Aberdeen Low Back Pain Questionnaire and back to work data. This could either be due to there being no functional improvement within the first three months, or if such small changes did exist, they may be too minute to be detected using the instruments chosen. Either way, the leaflet did not measurably influence clients' overall level of function. It could be argued that either sitting is not important; or it may only influence overall functional levels in clients who predominantly sit as part of their daily work; or something is acting as a barrier to prevent the significantly improved sitting posture being translated into an improved overall level of function.

\subsubsection{Sources of bias}

When considering any biases that may be evident in this study, the demographic details outlined in table 5.9 show that the social class of the experimental group was lower than the control group. This may have reduced any potential impact that may be gained from the leaflet, resulting in an underestimation of any true benefit.

The other main difference between the groups is the number of clients who had experienced previous episodes of low back pain (which was higher in the experimental group). This may have affected the clients' previous knowledge, having learnt how to manage from previous experience and therefore the results, as shown, may not be entirely due to the leaflet alone. Attempts had been made to minimize this source of bias by excluding clients who had received formal instruction in back care and also any low 
back pain in the previous six months to try and rule out recent experiences. However, despite these attempts, the difference (between these two groups) in previously experiencing low back pain, may lead to an overestimation of any true benefit of the leaflet.

G.Ps may have introduced a source of bias when they recruited clients into the 'Back Home' study. Although the standardized empowerment statement attempted to minimize this possible conscious or unconscious bias, no consultation will be identical and it must be remembered that the G.P. was fully aware of which study group they were in when they approached clients.

Another potential source of bias was the presence of the researcher at the home visits. Even though the majority of the outcome measures were self-report format, nevertheless, the researcher was an intervention, which could not be completely standardized, and therefore may have had some bearing on the results. Nevertheless, here again is the tension between doing practical versus ideal research. Removing the researcher would minimize this source of bias, but it is likely that changing the methods, for example to postal questionnaires, would have dramatically lowered the completion rates and the quality of data collected, partly because of the large number of questionnaires requested in this study. With the slow recruitment rate already occurring, this was not feasible.

\subsubsection{Limitations of the study}

The limitations of the leaflet were: a) using simple drawings instead of photographs, as advocated by $\mathrm{Moll}^{(23)}$ and Roux ${ }^{(21)}$; b) relatively small font size; and c) limited use of colour. Addressing such issues in future leaflets could aid clarity, impact and the aesthetic appearance of the leaflet.

Regarding the method, there were limitations in the outcome measures, level of randomization and sample size in this study.

Firstly, measuring knowledge is a complex issue. If it is measured as a baseline variable, it may cue clients to acquire specific information, and thus, when it is re-measured postintervention, bias has occurred. Therefore it should only be measured once. In this research, measuring knowledge only occurred during assessment two, so we have no information about clients' levels of knowledge regarding back pain prior to the 
intervention. Consequently, we cannot investigate any change in the level of clients' knowledge about back pain.

Secondly, limitations arose because of the level of randomization in this study. There were three possible alternatives: a) randomizing at the individual client level; b) at the individual G.P. level; or c) at the G.P. practice level. In choosing the most appropriate level of randomization, a number of factors must be considered.

Perhaps the most important aspect to consider is the empowerment process. In normal practice, a G.P. may give a client a leaflet and encourage them to adopt the content. This is only possible if the G.P. is aware that they are actually issuing a leaflet (and not a placebo). Therefore, this is only possible if randomization occurs at the level of the G.P. practice or individual G.P.

Sources of contamination also need to be considered. If only some clients in a practice receive a leaflet, due to randomization at either the individual client or G.P. level, then there is the possibility of contamination (e.g. from discussions between clients in the waiting room). Further sources of contamination may occur if clients return to see different G.Ps within the same practice, e.g. for a sickness certificate.

In addition, the likely impact on recruitment of clients by G.Ps into the study needs to be considered. If randomization occurs at either the individual G.P. or practice level, there is no incentive for G.Ps in the control group to recruit clients into the study.

Considering these factors, we decided, on balance, to randomize at the level of the G.P. practice, acknowledging the potential lack of incentive for control group G.Ps to recruit clients into the study. In the event this issue was under-estimated and resulted in two G.Ps withdrawing from the study (see chapter eight).

Finally, the results of this study should be interpreted with caution since the sample size is small ( $\mathrm{n}\{$ control $\}=28$ and $\mathrm{n}$ \{experimental $\}=35$ ). Nevertheless, it is encouraging that an educational intervention can change knowledge and behaviour in clients with acute low back pain. Unfortunately, this study was under-powered to draw firm conclusions about changes in function. Therefore the challenge remains of incorporating behavioural changes into a client's lifestyle to bring about an improved functional level. 


\subsection{Summary of the chapter}

- The 'Back Home' study was a single 'blind' randomized controlled trial to evaluate whether an optional G.P. diagnostic guide supplemented by a clientinformation leaflet improved the ability of people with acute low back pain to manage the episode of pain at home.

- The client-information leaflet was found to significantly affect clients' knowledge in relation to sitting posture and this knowledge was reflected in a change in behaviour which the client was unaware they were being observed on.

- These changes in knowledge and behaviour were not transposed into measurable improvements in clients' function. 


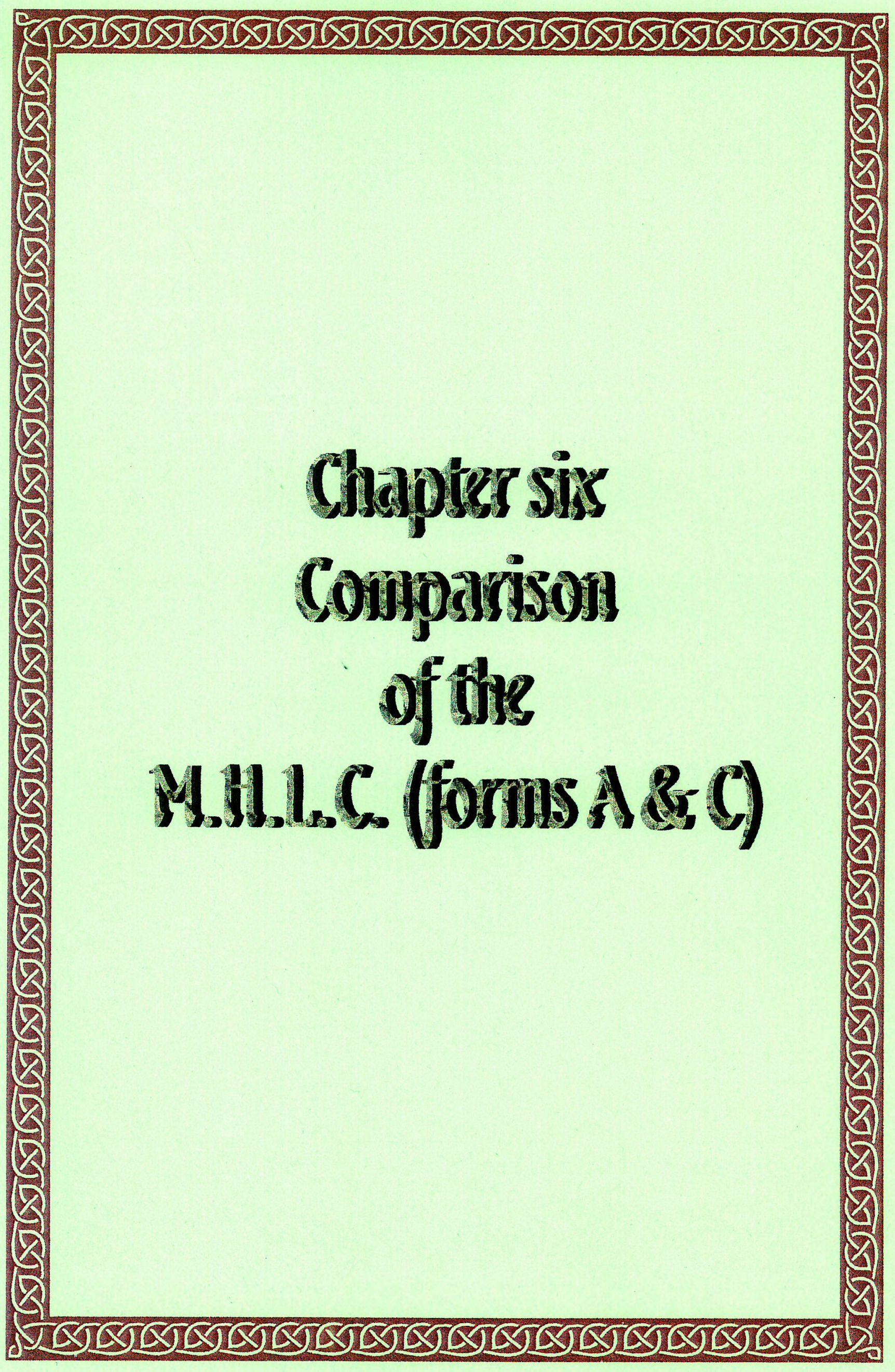




\section{Chapter six: Comparison of the M.H.L.C. (forms A \& C)}

As discussed in chapter four, there have been several attempts to measure the locus of control construct, using a variety of outcome measures.

Perhaps the most widely used measure in the health literature is the Multidimensional Health Locus of Control Scale (M.H.L.C.), devised by Wallston et al. ${ }^{(1)}$, which has already been described in section 4.8. More recently, the authors of this scale have devised a 'condition-specific' version (form C), which has not yet been used in a population of clients with acute low back pain.

Therefore, this chapter outlines the 'new', condition-specific measure and reports the findings of a simple comparison of this instrument, with the more established M.H.L.C. scale. This comparison was undertaken with clients participating in the 'Back Home' study. The analysis and interpretation of all the control outcomes over time are addressed in chapter seven - the work here exclusively reports on the instrument.

\subsection{Discovery of the condition-specific M.H.L.C. form C}

'There is a tide in the affairs of men,

Which, taken at the flood, leads on to fortune,

William Shakespeare (Julius Caesar) ${ }^{(2)}$

Despite the limitations of the M.H.L.C. form A, already outlined in section 4.8, it was decided that this instrument would be used to gain some insight into the locus of control of clients who were participating in the 'Back Home' study.

In 1994, I wrote to Professor Wallston at Vanderbilt University to seek permission to use the M.H.L.C. instrument, and advise him of our work. In his reply (appendix 6.1), he informed us that his team had developed a condition-specific version of the M.H.L.C. instrument, which, to date had not been used in clients with acute low back pain.

This correspondence was most fortunate as nothing had yet been published about this revised outcome measure in the literature, as it had only been in existence for about six months, and was currently in use in a variety of international studies. Unfortunately, since none of these studies appeared to involve clients with low back pain, it was decided 
that this measure would be used concurrently with the existing M.H.L.C. form A in our study, and thus the comparison of the two measures would take place alongside the 'Back Home' study.

\subsection{The M.H.L.C. (form C)}

Overall, the new form $\mathrm{C}$ was identical in structure to the existing form $\mathrm{A}$, the main difference was that the word 'health' was replaced with 'back pain', so that the concept of 'if I get sick ...' was replaced with 'if my back pain worsens ...' etc.

In preparing the new form $\mathrm{C}$ for use in our study, I noticed that questions one and six were identical, which was surprising. If the format of the two scales was to remain identical, question six should have been part of the internal locus of control subscale, but it was not clear why the wording was exactly the same. Therefore, before starting to use the scale in our study, I wrote to Professor Wallston again to seek clarification on this issue (appendix 6.2). His reply (appendix 6.3) suggests that the identical nature of questions one and six was due to typographical error, and thus the new form $\mathrm{C}$ was further revised, and a letter was sent to all researchers who were already using this scale (appendix 6.4).

A copy of the final version of the M.H.L.C. form C, as used in the 'Back Home' study can be seen in appendix 6.5 .

\subsection{The study}

After seeking statistical advice ${ }^{1}$, it was decided that the technique of split-half reliability coefficients (in S.P.S.S.) would be used to compare the M.H.L.C. form C with the established form A. This test is a measure of inter-consistency reliability that splits the data into two equal halves and once complete, the data are then correlated ${ }^{(3)}$, to give a coefficient value between 0 and 1 . The greater the positive correlation, the more reliable the test ${ }^{(3)}$.

\footnotetext{
${ }^{1}$ With thanks to Mrs S. High for her helpful advice.
} 
According to French, the odd numbered items are usually correlated with the even numbered items ${ }^{(3)}$, since 'it is not a good idea to correlate the first half of the test with the second half, as erroneous effects, such as lack of concentration or tiredness on the part of participants during the second half of the test, may be introduced ${ }^{\prime(3)}$. However, this is simplified in S.P.S.S., as the data are split in half so that the first half of the test (M.H.L.C. form C) is correlated with the second half (M.H.L.C. form A). This is evident as the reliability coefficient obtained from correlating the totals of the two M.H.L.C. scales is identical to the coefficient obtained if all 18 items on the M.H.L.C. form C are entered sequentially, followed by all 18 items on form A. Therefore, knowing that the data is handled in such as way means that French's reservations about the lack of concentration and tiredness do not apply to this study as questions $1-18$ on form $\mathrm{C}$ are correlated with questions $1-18$ on form A (as opposed to questions $1-8$ on both scales being correlated against questions $10-18$ ).

Having decided on the technique for comparing the scales, the analysis was undertaken on the following data:

- assessment one (completed at two working days from the initial G.P. visit);

- assessment three (at three months);

- assessment four (at six months);

- assessment five (at twelve months).

Assessment two (which was completed at two weeks) was not included, since only the M.H.L.C. form A data were collected at this time, due to the large number of other outcome measures that were already included in that assessment (see table 5.7), and thus split-half reliability coefficients could not be calculated. The results of the four assessments are presented in sections 6.3.1 - 6.3.4.

\subsubsection{Results from assessment one}

The results of the split-half reliability coefficients for the data collected at assessment one (completed within two working days of the client first visiting their G.P. with back pain) are summarized in table 6.1 . 


\section{Table 6.1: Split-half reliability coefficients for assessment one}

Results of the split-half reliability coefficients for the M.H.L.C. form C against the M.H.L.C. form A, in assessment one of the 'Back Home' study (i.e. within two working days of the clients' visit to their G.P.). The right-hand column shows the mean difference between M.H.L.C. form A and M.H.L.C. form C for each subscale. Number of valid cases $=60^{*}$.

\begin{tabular}{|l|c|c|c|}
\hline \multicolumn{1}{|c|}{ Scale } & $\begin{array}{c}\text { Number } \\
\text { of items }\end{array}$ & $\begin{array}{c}\text { Split-half reliability } \\
\text { coefficient }\end{array}$ & $\begin{array}{c}\text { M.H.L.C. } \\
\text { form A-form } \\
\text { C }\end{array}$ \\
\hline Total & 18 & 0.72 & -0.57 \\
\hline Internal subscale & 6 & 0.44 & 1.18 \\
\hline External (powerful others) subscale & 6 & 0.56 & -3.47 \\
\hline External (chance) subscale & 6 & 0.67 & 1.72 \\
\hline
\end{tabular}

* Although data were collected for 63 clients in assessment one, the M.H.L.C. form C was only available for 60 clients as data collection for the first two clients occurred whilst seeking clarification on the instrument design, and hence these data were not available. One other client did not complete the M.H.L.C. form $\mathrm{C}$ due to time constraints.

It might be anticipated that since the condition-specific M.H.L.C. form $\mathrm{C}$ instrument was developed directly from the established form A, that the split-half reliability coefficient would be considerably higher than 0.72 .

This lower-than-expected result suggests that clients feel differently about the ability to control their low back pain than they do about controlling general health. Anecdotally, evidence could be found when collecting the data during the study, that clients had given some thought to control issues for their back pain, for example, one client commented (whilst answering M.H.L.C. form C question 7):

'Although I think my low back pain is my own fault, it is not my fault if I have to bend and stretch [at work]'

Therefore, to try and understand why people feel differently about controlling low back pain than they do about other aspects of health, it is necessary to look at the split-half reliability coefficients of the three subscales of the M.H.L.C. scales. 
From table 6.1, it is clear that the greatest difference between clients' perceptions of the ability to control their low back pain and other aspects of health, was found in the internal subscale of the locus of control construct where the reliability coefficient was only 0.44 . The external (powerful others) subscale was in second place, and the least difference existed in the external (chance) subscale, as the reliability coefficient was 0.67 .

However, from these data, it was not possible to ascertain whether clients perceived their low back pain to be more controllable than other illnesses - the correlation coefficient just registers a difference. Therefore, it was necessary to look at the mean of the differences between forms $\mathrm{A}$ and $\mathrm{C}$, particularly noting the sign of any difference (rather than the magnitude). When this was done, the results showed that on the internal and external (chance) subscales, clients scored higher on form $A$ than form C (1.18 and 1.72 respectively). Thus, clients felt more able to control aspects of their general health than their low back pain, and perceived that chance had a greater influence in general health than in low back pain. (This is further discussed in chapter seven, when clients' attitudes to causative factors for their low back pain are considered.)

However, the reverse was true for the external (powerful others) subscale, where clients perceived that powerful others were more able to control low back pain, than general aspects of health $(-3.47)$. This finding was perhaps not surprising as there were two sources of bias, which were likely to have influenced this result. Firstly, assessment one took place within two working days of the client's visit to their G.P. (and thus the clients' recollections of their G.P. offering any advice, was likely to be fresh in their mind). Secondly, bias may result from the presence of the researcher (who had been contacted directly by the G.P. once they had approached the client to take part in the study and registered them). Both of these activities may have reinforced the role of powerful others (especially if the client's low back pain was also starting to resolve at this stage).

Having identified these issues, it was then necessary to analyse the remaining assessments, to see whether these trends were consistent over time. 


\subsubsection{Results from assessment three}

The results of the split-half reliability coefficients for the data collected at assessment three (completed at three months) are summarized in table 6.2.

\section{Table 6.2: Split-half reliability coefficients for assessment three}

Results of the split-half reliability coefficients for the M.H.L.C. form $\mathrm{C}$ against the M.H.L.C. form A, in assessment three of the 'Back Home' study (i.e. at three months follow-up). The right-hand column shows the mean difference between M.H.L.C. form A and M.H.L.C. form C for each subscale. Number of valid cases $=56^{*}$.

\begin{tabular}{|l|c|c|c||}
\hline \multicolumn{1}{|c|}{ Scale } & $\begin{array}{c}\text { Number } \\
\text { of items }\end{array}$ & $\begin{array}{c}\text { Split-half reliability } \\
\text { coefficient }\end{array}$ & $\begin{array}{c}\text { M.H.L.C. } \\
\text { form A-form C }\end{array}$ \\
\hline Total & 18 & 0.69 & -0.32 \\
\hline Internal subscale & 6 & 0.45 & -0.65 \\
\hline External (powerful others) subscale & 6 & 0.61 & -1.53 \\
\hline External (chance) subscale & 6 & 0.72 & 2.09 \\
\hline
\end{tabular}

"Although data were collected from 57 clients in assessment three, one client omitted one question in the M.H.L.C. form C, and hence it is excluded in the analysis.

From table 6.2 it can be seen that the split-half reliability coefficient for the total scores for M.H.L.C. forms $\mathrm{A}$ and $\mathrm{C}$ recorded at three months is very similar to that recorded for the first assessment (at two days).

Once again, the greatest difference between clients' perceptions of the ability to control their low back pain and other aspects of health, was found in the internal subscale of the locus of control construct, with external (powerful others) subscale in second place, and the least difference existed in the external (chance) subscale.

When looking more closely at the direction of difference between the subscale totals in forms $\mathrm{A}$ and $\mathrm{C}$, the same difference was observed as in assessment one. 'Clients perceived that chance had a greater influence in general health than in low back pain, and that the opposite was true for external (powerful others), i.e. powerful others were more able to control low back pain than general aspects of health.

However, interestingly, on the internal subscale, there had been a shift in clients' perceptions, so that now they perceived themselves as more able to control their low 
back pain than they could control their general health $(-0.65)$. This was opposite to their perceptions recorded at assessment one (1.18). The most obvious explanation for this shift is that the intensity of pain (recorded on a visual analogue scale) had decreased substantially during the three month period, and thus clients felt more able to control their pain when it was less intense. (The mean visual analogue score for the intensity of pain at assessment one $=54.0$, as compared with 16.8 at assessment three). Due to the time difference between assessments one and three, it is not possible to establish exactly when this shift in internal locus of control occurred during this three month period.

\subsubsection{Results from assessment four}

The results of the split-half reliability coefficients for the data collected at assessment four (completed at six months) are summarized in table 6.3.

\section{Table 6.3: Split-half reliability coefficients for assessment four}

Results of the split-half reliability coefficients for the M.H.L.C. form $\mathrm{C}$ against the M.H.L.C. form A, in assessment four of the 'Back Home' study (i.e. at six months follow-up). The right-hand column shows the mean difference between M.H.L.C. form A and M.H.L.C. form C for each subscale. Number of valid cases $=48$.

\begin{tabular}{|l|c|c|c|}
\hline \multicolumn{1}{|c|}{ Scale } & $\begin{array}{c}\text { Number } \\
\text { of items }\end{array}$ & $\begin{array}{c}\text { Split-half reliability } \\
\text { coefficient }\end{array}$ & $\begin{array}{c}\text { M.H.L.C. } \\
\text { form A-form C }\end{array}$ \\
\hline Total & 18 & 0.83 & -0.02 \\
\hline Internal subscale & 6 & 0.63 & -0.38 \\
\hline External (powerful others) subscale & 6 & 0.66 & -1.27 \\
\hline External (chance) subscale & 6 & 0.81 & 1.63 \\
\hline
\end{tabular}

From table 6.3 it can be seen that the split-half reliability coefficient for the total scores for M.H.L.C. forms A and C has increased to 0.83 from that calculated at three months, (0.69). However, it must be remembered that the number of valid cases in this analysis had decreased from 56 in assessment three to 48 in assessment four, which may have influenced this result. The reason that the attrition rate reached its peak between three and six months was that the formal period of data collection for the 'Back Home' study was complete, and clients were asked if they would be willing to continue the study for a further six months, and six declined. In addition, one client was unable to complete both 
M.H.L.C. instruments during assessment four due to time constraints, and on one other occasion, a home visit was terminated by the researcher for safety reasons. Thus, accepting this difference in the number of valid cases, by six months, the difference between how clients perceive the controllability of their back pain when compared with the controllability of their general health, had lessened in comparison with the earlier assessments. (The split-half reliability coefficient for the total scores of forms A and C had increased to 0.83.)

This time, the internal subscale of the locus of control construct was converging between the two M.H.L.C. forms, suggesting that by six months the large difference previously seen in how clients perceived their control over their back pain versus their control over general health issues, had lessened. In fact, the split-half reliability coefficient for the internal subscale was almost identical to that of the external (powerful others) subscale, and once again, the least difference existed in the external (chance) subscale.

As previous, the direction of difference between the subscale totals in forms $\mathrm{A}$ and $\mathrm{C}$ was studied, and found to be identical to assessment three i.e. clients perceived themselves and powerful others as more able to control their low back pain than their general health, whereas chance had a greater influence in general health than low back pain. Again the mean intensity of pain recorded on the visual analogue scale was low 13.6, suggesting that in the majority of cases, clients' back pain had resolved by six months.

\subsubsection{Results from assessment five}

The results of the split-half reliability coefficients for the data collected at assessment five (completed at twelve months) are summarized in table 6.4. 


\section{Table 6.4: Split-half reliability coefficients for assessment five}

Results of the split-half reliability coefficients for the M.H.L.C. form C against the M.H.L.C. form A, in assessment five of the 'Back Home' study (i.e. at twelve months follow-up). The right-hand column shows the mean difference between M.H.L.C. form A and M.H.L.C. form C for each subscale. Number of valid cases $=48$.

\begin{tabular}{|l|c|c|c|}
\hline \multicolumn{1}{|c|}{ Scale } & $\begin{array}{c}\text { Number } \\
\text { of items }\end{array}$ & $\begin{array}{c}\text { Split-half } \\
\text { reliability } \\
\text { coefficient }\end{array}$ & $\begin{array}{c}\text { M.H.L.C. } \\
\text { form A-form C }\end{array}$ \\
\hline Total & 18 & 0.79 & -0.73 \\
\hline Internal subscale & 6 & 0.65 & -0.35 \\
\hline External (powerful others) subscale & 6 & 0.68 & -1.69 \\
\hline External (chance) subscale & 6 & 0.72 & 1.31 \\
\hline
\end{tabular}

The results of the split-half reliability coefficient for the total scores for the M.H.L.C. forms $\mathrm{A}$ and $\mathrm{C}$ were similar to those reported in assessment four.

When considering the coefficients for the three subscales in assessment five (shown in table 6.4), the values obtained were very similar to each other i.e. there was not the 'gap' between the value of the internal subscale and the other two that had been evident in the early assessments. In assessment four (section 6.3.3), it was proposed that by six months this large difference in how clients perceived their control over their back pain versus their control over general health issues, had lessened. Since the split-half reliability coefficient for the internal subscale in assessments four and five is almost identical, this trend remains consistent between six and twelve months.

Furthermore, the direction of difference between the subscale totals in forms A and C were identical in assessment five to assessment four i.e. clients perceived themselves and powerful others to be more able to control their low back pain than their general health, whereas chance had a greater influence in general health issues than low back pain.

\subsection{Summary of findings}

Having examined the data collected at two days, three months, six months and one year using the M.H.L.C. forms A and C, several trends can be seen: 
1. The most striking result is the change in split-half reliability coefficients for the internal locus of control subscale in the different assessments. Initially, the coefficient is low ( 0.44 in assessment one and 0.45 in assessment three), which suggests that clients perceive their ability to control their low back pain as considerably different to their ability to control their general health. However, between three and six months, there is a shift in clients' perceptions as the split-half reliability coefficient increases to 0.63 , suggesting that clients' perceptions of their ability to control their low back pain and their general health are converging.

2. Initially, in assessment one, clients felt more able to control aspects of their general health than their low back pain. However, by three months, this trend had reversed, and throughout the remainder of the assessments, clients felt more able to control their low back pain by their own actions, than their general health.

3. Throughout all four assessments, the split-half reliability coefficient for 'external chance' was the highest of the three subscales. This means that how clients perceive chance factors to affect their back pain was similar to how they perceived chance factors to affect their general health.

4. As time passed, the subscale scores converged, suggesting that the three domains of the locus of control construct (internal, external [powerful others], and external [chance]), are not mutually exclusive i.e. an increase on the internal scale does not automatically result in a decrease on the external scales, and vice versa.

5. Attrition rates for this study were greatest between three and six months due to the study design and practical issues (including time constraints and safety issues).

\subsection{Implications of findings}

Firstly, this study shows the importance of testing any new or modified instrument against established measures. Researchers cannot just change the wording in established outcome measures and assume that these changes have only tailored the measure to their needs. Any changes must be tested.

However, the notion of comparing an instrument with a 'gold standard' is debatable, since the reason that the gold standard instrument is not being used for a particular piece 
of research, is that the researchers perceive that it is not entirely suitable for their purpose. Therefore, the new or revised instrument will differ in some way from the gold standard, and so it is not surprising that there will be some differences in the validity and reliability of the two instruments. Nevertheless, it is important to establish the magnitude of these differences, and to attempt to gain some understanding of the psychometric properties of the new measure. Another important consideration in comparison studies is that they can only be undertaken longitudinally, and not done retrospectively, otherwise, subtle changes may be missed, and inaccuracies may result from participants' lapses in memory.

Despite these complexities, another important implication of the results from this chapter, is that clients' perceptions about their back pain and their general health are not synonymous. It is essential to look at these perceptions within the specific context, for example, it was important to consider the natural history of resolution of back pain and the reported pain intensities for clients in the 'Back Home' study, to try and gain insight into the changes on the internal locus of control subscale.

Having purported that clients' perceptions about their low back pain are not synonymous with perceptions about their general health, based on the findings of this comparison, it would be more appropriate to use the M.H.L.C. form $\mathrm{C}$ in future low back pain studies which involve measuring the locus of control concept, in preference to form A (or form B). However, this conclusion should be considered with caution, as the author's reservations about the locus of control construct (identified in chapter two), and the reservations about its measurement (expressed in chapter four) still hold true.

Nevertheless, in real world research, where locus of control may still have a place, a condition-specific measurement tool could help explore these complex, psychosocial characteristics. 


\subsection{Summary of the chapter}

- If new outcome measures are developed, or existing ones modified, then the revised instruments must be tested before they can be used in research studies.

- The three subscales of the locus of control construct: internal; external (powerful others); and external (chance), are not mutually exclusive i.e. an increase on one scale does not automatically result in a decrease on the other scales.

- If clients' perceptions of their controllability of their low back pain is being studied, it is preferable to use the condition-specific M.H.L.C. form C scale, rather than the more general M.H.L.C. form A, because people's perceptions of the controllability of their general health and their back pain are not directly comparable. 


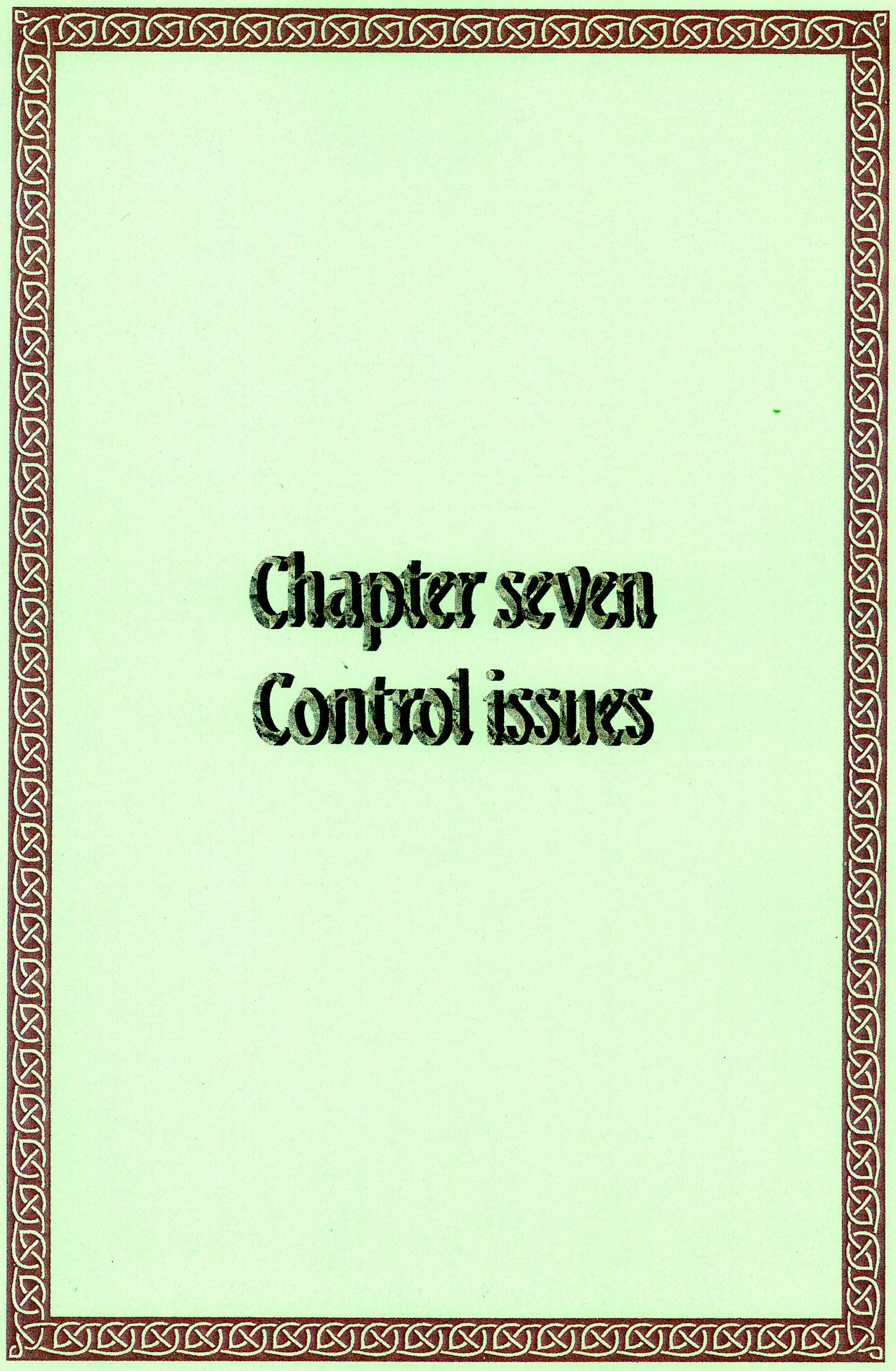




\section{Chapter seven: Control issues}

The aim of this thesis is to study control issues in clients with acute low back pain, building on the literature summarized in chapter two, and using the instruments for measuring control which have already been discussed in chapter four.

This work has run concurrently with the 'Back Home' study (described in chapter five), where clients were followed-up over three months, with primary outcome measures of knowledge, attitude, observable behaviour and function. However, a time span of three months was considered too short to grasp how clients' perceptions of control change over time, and therefore, for the purpose of studying these control issues, the follow-up period was extended, with two further visits, at six months and one year.

This chapter specifically addresses the results of the control outcomes (locus of control and perceived control) over one year, separate from the main 'Back Home' study, and is primarily focused on changes in clients' perceptions of control over time. In addition, a secondary analysis is undertaken which explores the influence of clients' attitude (about the cause of their back pain), anxiety and satisfaction on these control concepts. The chapter concludes by discussing the limitations of actually measuring control in clinical practise.

\subsection{Research question}

From the literature review in chapter two, no longitudinal studies could be found which reported changes in perceptions of control in clients with acute low back pain over time. Therefore, before the influence of other factors (such as attitude, anxiety, and satisfaction) could be investigated, a base-line needed to be established for clients' perceptions of control.

Having identified a need for this investigation, the simple research question asked 'How do perceptions of control change during a one year period in a population of clients with acute low back pain?' This question suggested that some change would take place, but it did not make any assumptions about the direction of any such change. 


\subsection{Multidimensional Health Locus of Control perceptions over time}

From chapter six, it was concluded that clients' perceptions about their low back pain were not synonymous with their perceptions about their general health. Therefore, in this analysis, the condition-specific, M.H.L.C. (form C) was used, which specifically addressed control issues in low back pain. To recap, the scale contains 18 items and each response involves selecting one of six choices on a Likert scale, ranging from 'strongly disagree' (scoring 1), to 'strongly agree' (scoring 6).

The first stage in the analysis was to calculate measures of central tendency for the M.H.L.C. (form C) in each of the four assessments that used this outcome measure. These results are presented in table 7.1 .

\section{Table 7.1: Measures of central tendency for the M.H.L.C. (form C)}

This table shows the means (and standard deviations in parentheses) for the M.H.L.C. (form C) total and subscales, in assessments 1,3,4 and 5. The 'powerful others' results are presented as a subscale and then sub-divided as suggested by Wallston ${ }^{(1)}$. As the median results were very similar to the mean, for clarity, these figures are omitted.

\begin{tabular}{|l|ll|ll|ll|ll||}
\hline & $\begin{array}{c}\text { Assessment 1 } \\
\mathrm{n}=60\end{array}$ & \multicolumn{2}{|c|}{$\begin{array}{c}\text { Assessment 3 } \\
\mathrm{n}=56\end{array}$} & \multicolumn{2}{|c|}{$\begin{array}{c}\text { Assessment 4 } \\
\mathrm{n}=48\end{array}$} & \multicolumn{2}{|c|}{$\begin{array}{c}\text { Assessment 5 } \\
\mathrm{n}=48\end{array}$} \\
\hline Total & 63.6 & $(10.7)$ & 63.9 & $(10.3)$ & 63.0 & $(10.7)$ & 64.1 & $(12.5)$ \\
\hline Internal & 23.3 & $(5.6)$ & 24.9 & $(4.4)$ & 23.9 & $(5.0)$ & 24.3 & $(4.7)$ \\
\hline Powerful others & 22.3 & $(4.2)$ & 20.3 & $(4.8)$ & 20.2 & $(5.0)$ & 20.3 & $(5.2)$ \\
Powerful others (Doctors) & 11.8 & $(2.7)$ & 10.7 & $(2.7)$ & 10.5 & $(2.9)$ & 10.6 & $(2.8)$ \\
Powerful others (others) & 10.5 & $(2.5)$ & 9.6 & $(3.1)$ & 9.7 & $(3.0)$ & 9.6 & $(3.1)$ \\
\hline Chance & 18.0 & $(5.4)$ & 18.5 & $(5.7)$ & 18.9 & $(5.7)$ & 19.5 & $(5.6)$ \\
\hline
\end{tabular}

From table 7.1, it is evident that the results of the mean scores for each subscale and the totals vary little over time, when the size of the standard deviation is taken into consideration. 
Generally, the trend exists that the mean internal subscale scores are consistently higher than the external (powerful others) scores, which are higher than the external (chance) subscale scores. This suggests that in this population of clients with acute low back pain, using the M.H.L.C. (form C), clients perceived themselves to be more able to influence their back pain than powerful others, with chance being the least influential factor. The results are presented graphically, as box-and whisker plots, in figures 7.17.5 .

\section{Figure 7.1: Results of the M.H.L.C. (form C) - total scores}

The chart shows the total scores of the M.H.L.C. (form C), from assessments 1,3,4 and 5, i.e. clients' perceptions of their own ability, powerful others and chance to influence their current episode of low back pain.

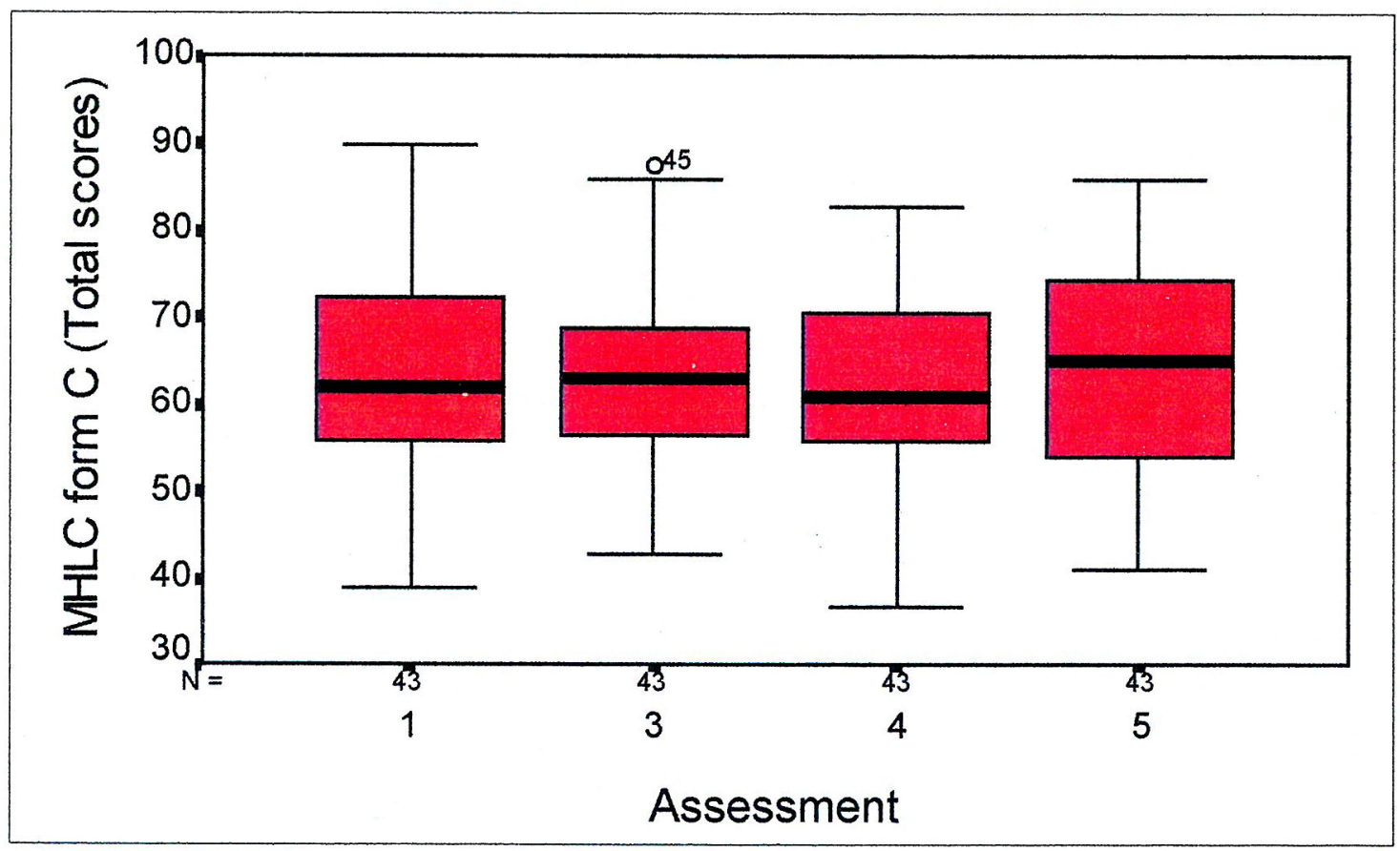

In the box-plots, the lower boundary of the box itself marks the $25^{\text {th }}$ percentile, while the upper boundary represents the $75^{\text {th }}$ percentile, and so the length of the box indicates the inter-quartile range ${ }^{(2)}$. The dark band is the median, and the whiskers mark the largest and smallest values (excluding outliers) ${ }^{(2)}$.

From the plots showing the results of the M.H.L.C. (form C) total scores (figure 7.1) and the internal scores (figure 7.2), it can be seen that the scores remain relatively consistent across the four assessments, with no remarkable features. 


\section{Figure 7.2: Results of the M.H.L.C. (form C) - internal scores}

The chart shows the internal subscale scores of the M.H.L.C. (form C), from assessments $1,3,4$ and 5 .

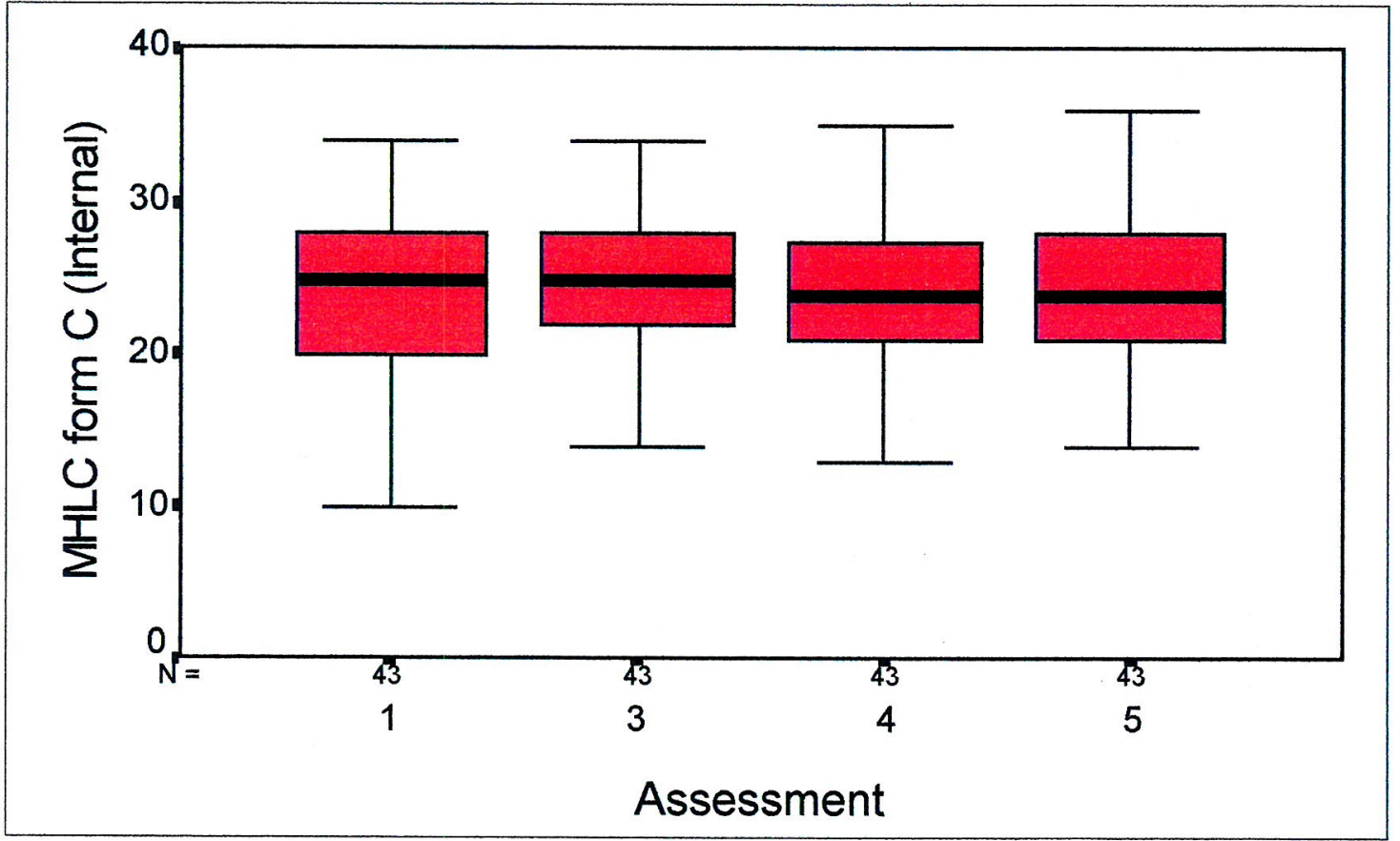

When considering the results from the external (powerful others) subscale, it is perhaps not surprising, given the role of the G.P. in recruiting clients into the study, that the powerful others (doctors) subscale scored slightly higher than the powerful others (others) subscale, shown in figures 7.3 and 7.4.

What is particularly interesting is that client number ' 62 ' was an outlier ${ }^{1}$ in assessment one on the 'powerful others - doctors' subscale, only scoring 4 out of a possible 18 , and yet was also an outlier in assessment five on the 'powerful others - others' subscale, but this time for scoring 17 out of a possible 18. It is clients like this who support Wallston's notion of subdividing the external (powerful others) scale into 'doctors' and 'others ${ }^{\text {(1) }}$, since clearly, clients can have differing perceptions of all, so called 'powerful others'.

\footnotetext{
${ }^{1}$ By definition, an outlier is a case with values between 1.5 and 3 box lengths from the upper or lower edge of a box ${ }^{(2)}$.
} 
Figure 7.3: Results of M.H.L.C. (C) (powerful others - 'doctors' scores) The chart shows the powerful others (doctors) scores of the M.H.L.C. (form C), from assessments $1,3,4$ and 5 .

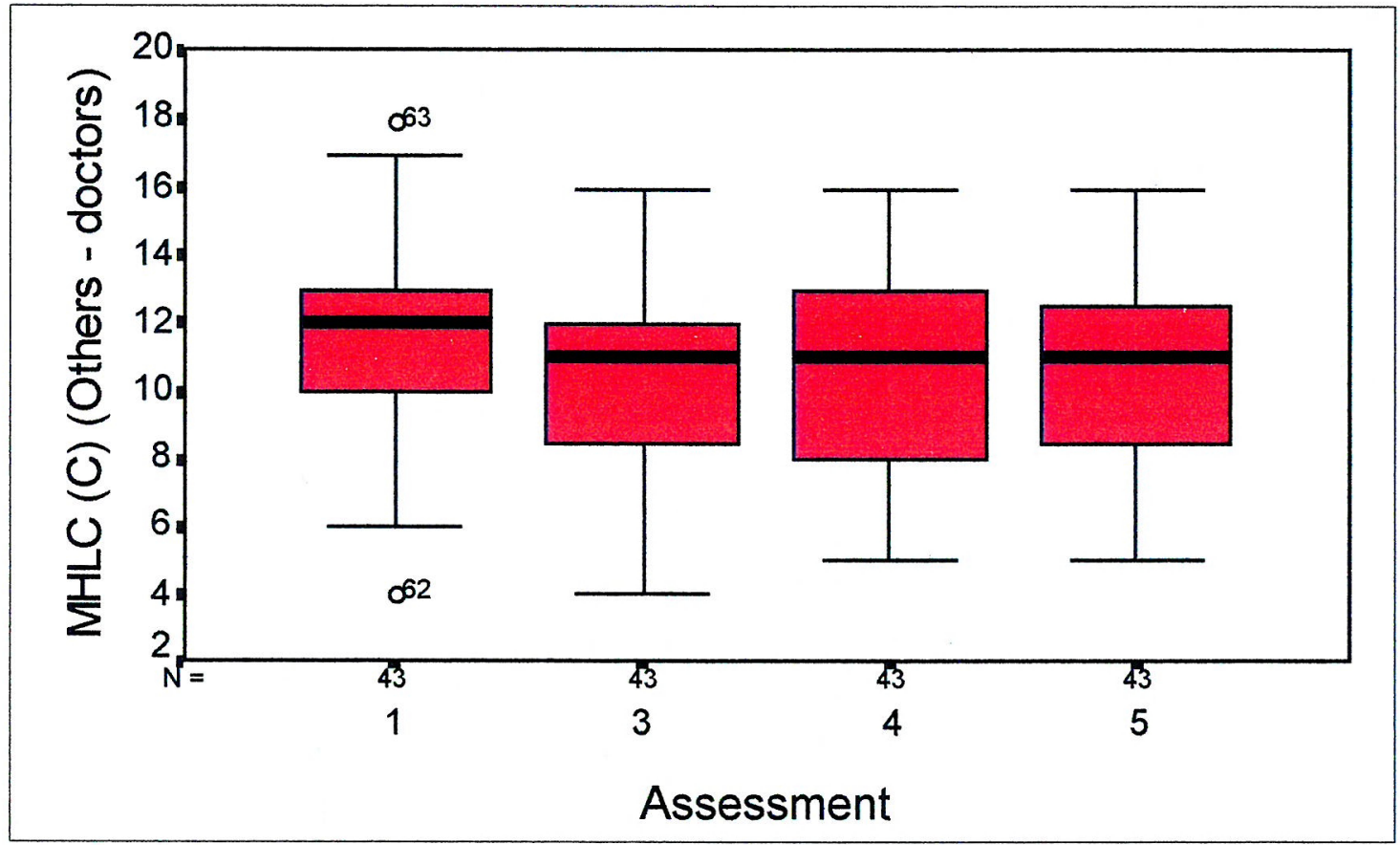

Figure 7.4: Results of M.H.L.C. (C) (powerful others - 'others' scores) The chart shows the powerful others (lay) scores of the M.H.L.C. form C, from assessments $1,3,4$ and 5 .

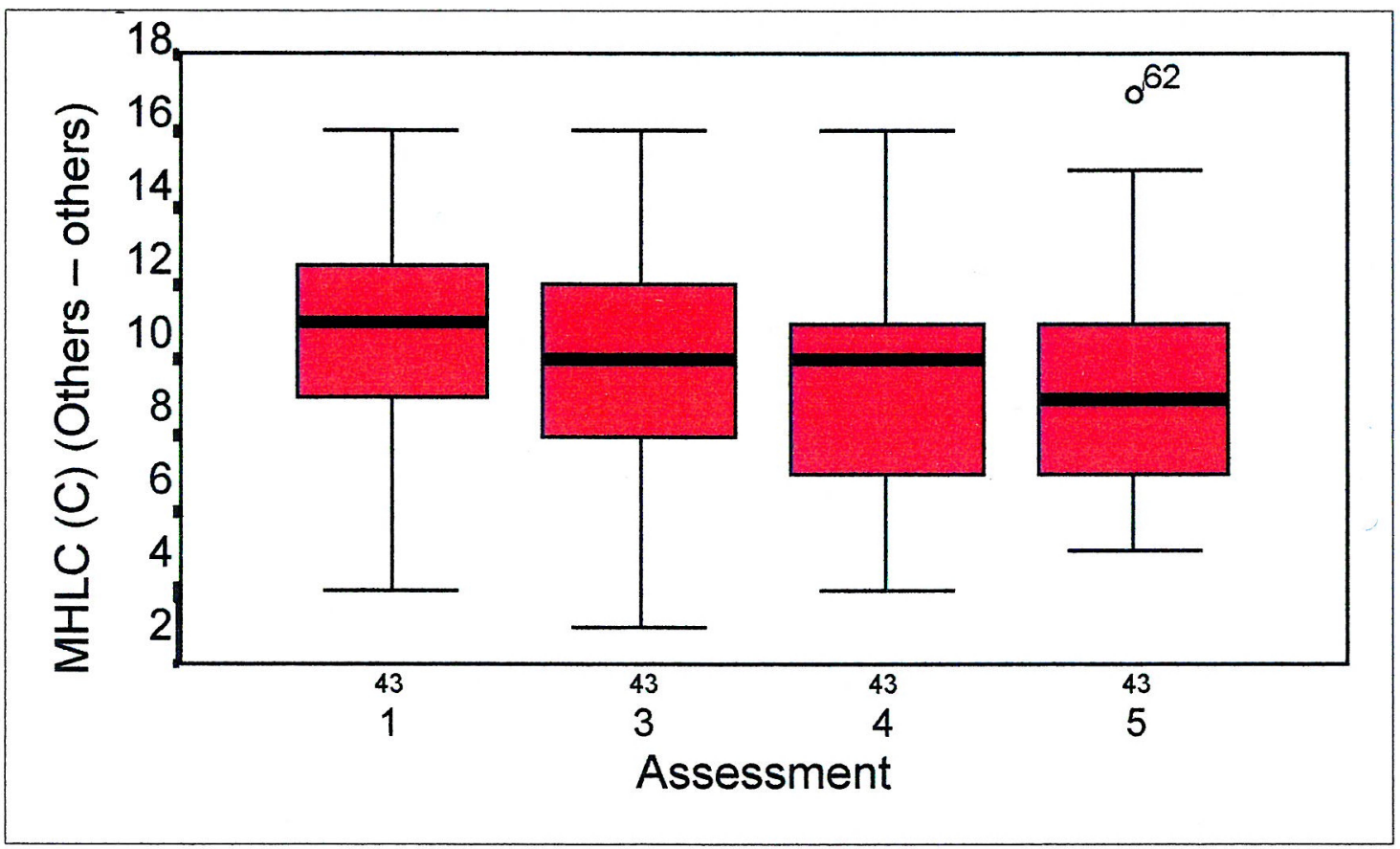


Figure 7.5: Results of the M.H.L.C. (C) (external chance scores)

The chart shows external chance scores of the M.H.L.C.(C), from assessments 1,3,4 and 5.

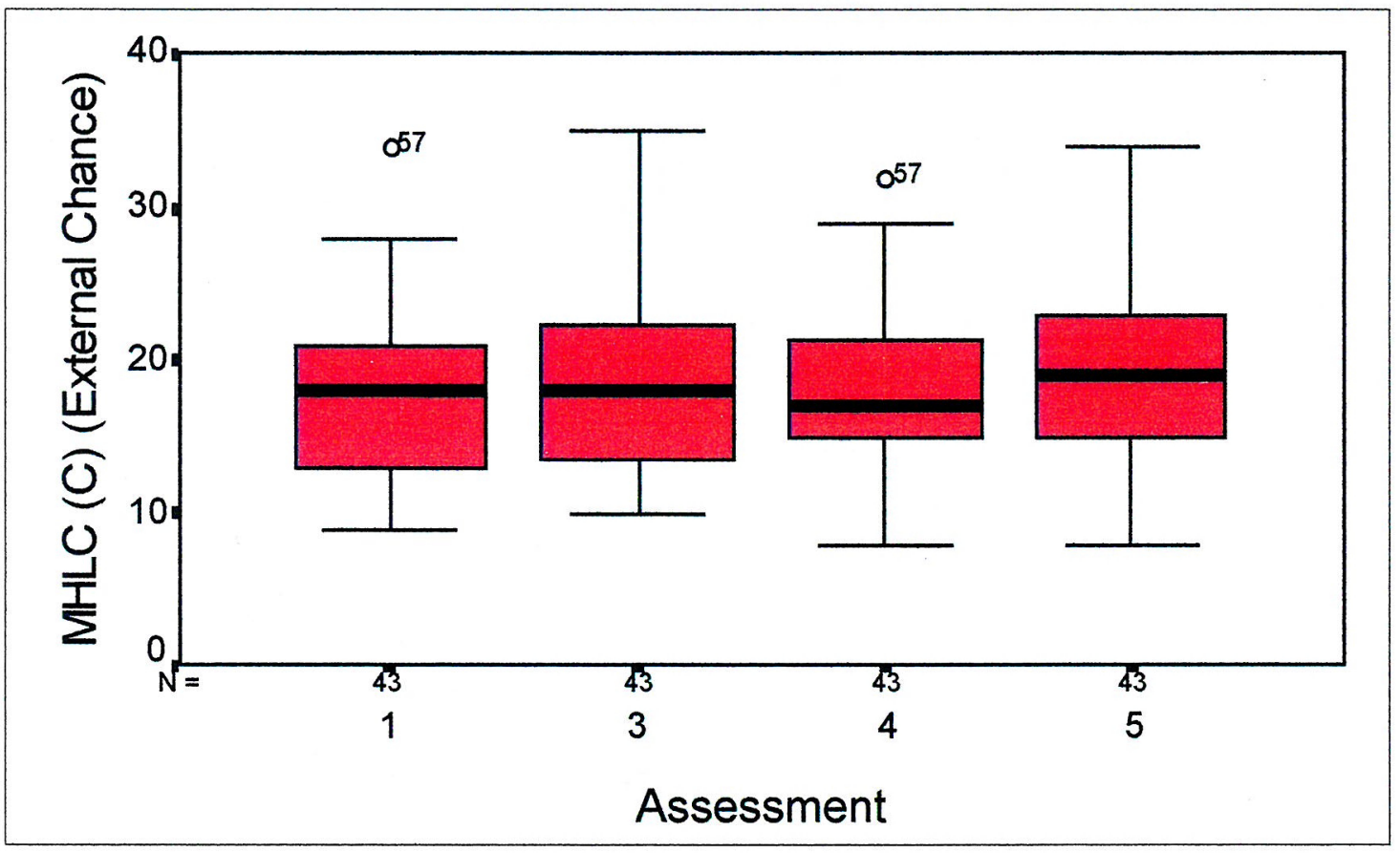

Although this analysis was concerned with the client population as a whole, it must be remembered from chapter five that some of the clients (i.e. those in the experimental group) had received a copy of the 'Back Home' leaflet, whilst the clients in the control group had not, and thus any impact of this leaflet needed to be considered.

The means of the M.H.L.C. (form C) for the experimental and control groups were compared and analysed using non-parametric statistics (as the Likert scale data were ordinal). The results of assessment one are presented in table 7.2.

From table 7.2 , it can be seen that there was a significant difference (at the 0.05 level) in the total score and the internal subscale between the experimental and control group. Thus, at two days, clients who had received a leaflet perceived themselves as being significantly more able to control their low back pain than those who had not received the leaflet. 


\section{Table 7.2: M.H.L.C.(C) scores for the experimental and control groups}

The table shows the results of the Mann-Whitney statistics for the M.H.L.C. (form C) collected at assessment one (two days), subdivided into the experimental and control groups. ${ }^{*}=$ significant at the 0.05 level.

\begin{tabular}{|l|c|c|c||}
\hline & $\begin{array}{c}\text { Experimental } \\
\text { group (n=35) }\end{array}$ & $\begin{array}{c}\text { Control } \\
\text { group (n=28) }\end{array}$ & p value \\
\hline Total & 66.3 & 60.3 & $0.038^{*}$ \\
\hline Internal & 24.8 & 21.6 & $0.039^{*}$ \\
\hline Powerful others & 22.5 & 22.0 & 0.823 \\
Powerful others (doctors) & 12.2 & 11.2 & 0.159 \\
Powerful others (others) & 10.2 & 10.8 & 0.475 \\
\hline Chance & 19.1 & 16.7 & 0.080 \\
\hline
\end{tabular}

When similar analyses were completed for assessments three, four and five, there were no significant differences between the two groups, and hence the tables are omitted. Possible reasons for this shift in perceptions from assessment one could be: i) that the low back pain was resolving in both client groups by assessment three (three months); ii) clients who had received the leaflet did not retain the content and apply it to their daily lives; or iii) clients in the control groups had gained personal control over their low back pain through means other than the leaflet (such as media sources, friends, family etc.). From the data collected, the precise reasons for this shift in perceptions cannot be determined, although the most likely influence was probably the natural history of resolution of acute low back pain.

\subsubsection{Influence of gender on M.H.L.C. perceptions}

In addressing the research question of how clients' perceptions of control change during a one-year period, it was necessary to consider the relevance of gender, as this was identified as an influential factor in the control literature in section 2.14. Härkäpää et al. reported findings from a survey of 459 people with chronic and recurrent low back pain, and they concluded that 'men were more internal in their beliefs' ${ }^{\text {'(3). }}$ 
Härkäpää et al. were not alone in their findings, as Tait et al. reported that women with chronic low back pain in the United States and New Zealand, rated themselves as having less personal control over health than men, using the Health Locus of Control Scale ${ }^{(4)}$. Tait's sample is not directly comparable to the clients in the 'Back Home' study for three reasons: i) Tait's study did not take place in the U.K.; ii) clients in this study had experienced chronic as opposed to acute low back pain; and iii) Tait did not use the M.H.L.C. (form C) scale. Nonetheless, a simple analysis was needed to determine whether the trend in personal control, identified by Tait et al., was consistent with this sample.

Therefore, in assessments one, three, four and five, the totals of the M.H.L.C. (form C) subscales (internal, powerful others [doctors and others], and chance) were compared between males and females. The analysis used non-parametric statistics (as the Likert scales were ordinal), and the results are presented in table 7.3.

\section{Table 7.3. The influence of gender on perceptions of control}

The table shows the results of the Mann-Whitney statistics for the M.H.L.C. (form C) collected at assessments $1,3,4$ and $5 .{ }^{*}=$ significant at the 0.05 level.

\begin{tabular}{|l|c|c|c|c||}
\hline & $\begin{array}{c}\text { Assessment 1 } \\
\mathrm{M}=39 \mathrm{~F}=21\end{array}$ & $\begin{array}{c}\text { Assessment 3 } \\
\mathrm{M}=38 \mathrm{~F}=19\end{array}$ & $\begin{array}{c}\text { Assessment 4 } \\
\mathrm{M}=32 \mathrm{~F}=16\end{array}$ & $\begin{array}{c}\text { Assessment 5 } \\
\mathrm{M}=31 \mathrm{~F}=17\end{array}$ \\
\hline Internal & 0.066 & 0.059 & 0.653 & 0.198 \\
\hline Powerful others (doctors) & $0.010^{*}$ & $0.046^{*}$ & 0.055 & $0.004^{*}$ \\
Powerful others (others) & 0.863 & 0.233 & 0.073 & $0.023^{*}$ \\
\hline Chance & 0.786 & 0.404 & $0.018^{*}$ & $0.007^{*}$ \\
\hline
\end{tabular}

When the results in table 7.3 are studied, there are two notable trends:

- For both the powerful others (others) and chance subscales, the differences between males and females become more marked with time (as the MannWhitney scores decrease substantially).

- There are several significant differences between male and female perceptions of control on the M.H.L.C. (form C) external subscales. 
The results from the internal subscale are variable, and not consistent with Tait et al. 's findings. In this sample, the differences between men and women's perceptions of control were more pronounced on the external subscales than on the internal subscale.

On further investigation of the data, it was the male clients who scored more highly on all the external subscales, suggesting that they perceived a greater influence of powerful others and chance factors in their acute low back pain than the female clients.

Throughout this analysis, it must be remembered that the ratio of male to female clients was around 3:2, and the sample size was relatively small. However, despite these caveats, gender issues are clearly pertinent to health-related perceptions of control, and warrant consideration in any further investigations in this area.

\subsubsection{Influence of age on M.H.L.C. perceptions}

In the literature review, age was another factor that was considered influential on clients' perceptions of control, although the reported findings have not been consistent ${ }^{(3)}$. Smith had previously hypothesized that the magnitude of change from externality towards (relative) internality would be negatively related to age, but the results of his study, with crisis and non-crisis clients presenting at a neuro-psychiatric emergency centre, did not support this hypothesis ${ }^{(5)}$. In contrast, Härkäpää et al. did report that 'younger subjects were more internal in their beliefs ${ }^{(3)}$, despite the age of the 'younger subjects' not being defined in this sample of people aged 35-54 years (although the 'oldest group' was defined as 50 years or older $\left.{ }^{(3)}\right)$.

In the 'Back Home' study, the age of clients (when they were entered into the study) ranged from 17 to 59 years, with a mean of 39.7 years and standard deviation of 10.3 years. The frequencies of their ages are summarized in table 7.4. 


\section{Table 7.4: Ages of participating clients}

The table shows the ages of clients who participated in the 'Back Home' study $(n=63)$.

\begin{tabular}{|c|c|}
\hline Age & Frequency \\
\hline $10-19$ & 1 \\
\hline $20-29$ & 11 \\
\hline $30-39$ & 20 \\
\hline $40-49$ & 17 \\
\hline $50-59$ & 14 \\
\hline
\end{tabular}

When the results of the M.H.L.C. (form C) subscales were compared in the different age groups, the findings were unremarkable, and did not support either Smith's hypothesis ${ }^{(5)}$ or Härkäpää et al.'s findings ${ }^{(3)}$.

Had the trend of decreasing internality with increasing age been evident in this sample, it should theoretically have been even stronger than Härkäpää et al. suggested due to the wider age range studied, but no such trend was seen, and it would be unreasonable to speculate why this trend did not occur.

\subsubsection{Summary of M.H.L.C. (form C) perceptions over time}

From all the information presented in section 7.2 , four main points emerge:

- In the population of clients with acute low back pain in this study, using the condition-specific M.H.L.C. (form C), generally the internal subscale scores are consistently higher than the external (powerful others) scores, which in turn are higher than external (chance) subscale scores, across all assessments. This suggests clients perceive themselves as more able to influence their back pain than powerful others. Chance factors are the least influential of the three factors.

- In assessment one (completed at two days), clients who had received a 'Back Home' leaflet scored more highly on the internal subscale of the M.H.L.C. (form C) and thus, perceived themselves more able to control their low back pain than those who had not received the leaflet. (This result was significant at the 0.05 level.) This significant difference was not maintained in subsequent assessments. 
- Clear differences exist between males and females perceptions of control, with males scoring more highly on all the external subscales of the M.H.L.C. (form C) than females. This suggests that male clients perceived a greater influence of powerful others and chance factors in their acute low back pain than the female clients.

- Age factors did not influence clients' perceptions of control in this study.

It is important to remember that although these trends were seen, the reservations expressed about the locus of control theory and its measurement (in chapters two and four) are still valid, and thus these results should be interpreted with some caution.

\subsection{Perceived control perceptions over time}

Having considered the results from the main control outcome measure (M.H.L.C. (form C)), it was necessary to consider the results from the perceived control instrument, despite its poor psychometric properties, discussed in section 5.8 (where the reliability coefficient was only $53 \%$ ).

With such a poor outcome measure, it is not appropriate to present a detailed analysis and individual results, for all questions in the five assessments. However it suffices to say that a few trends were seen, which are summarized in figure 7.6.

From the graph, it is evident that clients' perceived influence of chance on the cause of their back pain decreased with time. Meanwhile, the perceived influence of: something about themselves; other people or circumstances; whether their back pain was controllable by them; and whether they could have foreseen the cause of their back pain; increased with time.

The results of the perceived 'chance' findings appear at first sight to contradict the trend seen with the external (chance) subscale of the M.H.L.C. (form C) - see table 7.1. However, in both cases, the standard deviation is large, and the instrument is not robust, and thus any such conclusions would be invalid. In summary, little can be concluded with this instrument. 


\section{Figure 7.6: Graph of perceived control versus time}

The graph shows the results of the perceived control instrument for assessments $1,2,3,4$ and 5 (with $\mathrm{n}=63,58,57,49$ and 48 respectively). The questions asked 'To what extent ...:

Self $\quad=$ ' $\ldots$ was the cause of your back pain something about you?'

Others $\quad=$ ' $\ldots$ was the cause something to do with other people or circumstances?'

Chance $\quad=$ ' $\ldots$ was the cause due to chance?'

Controllable $=$ ' $\ldots$ is your back pain controllable by you?'

Foreseeable $=$ '... do you think you could have foreseen the cause of your back pain?'

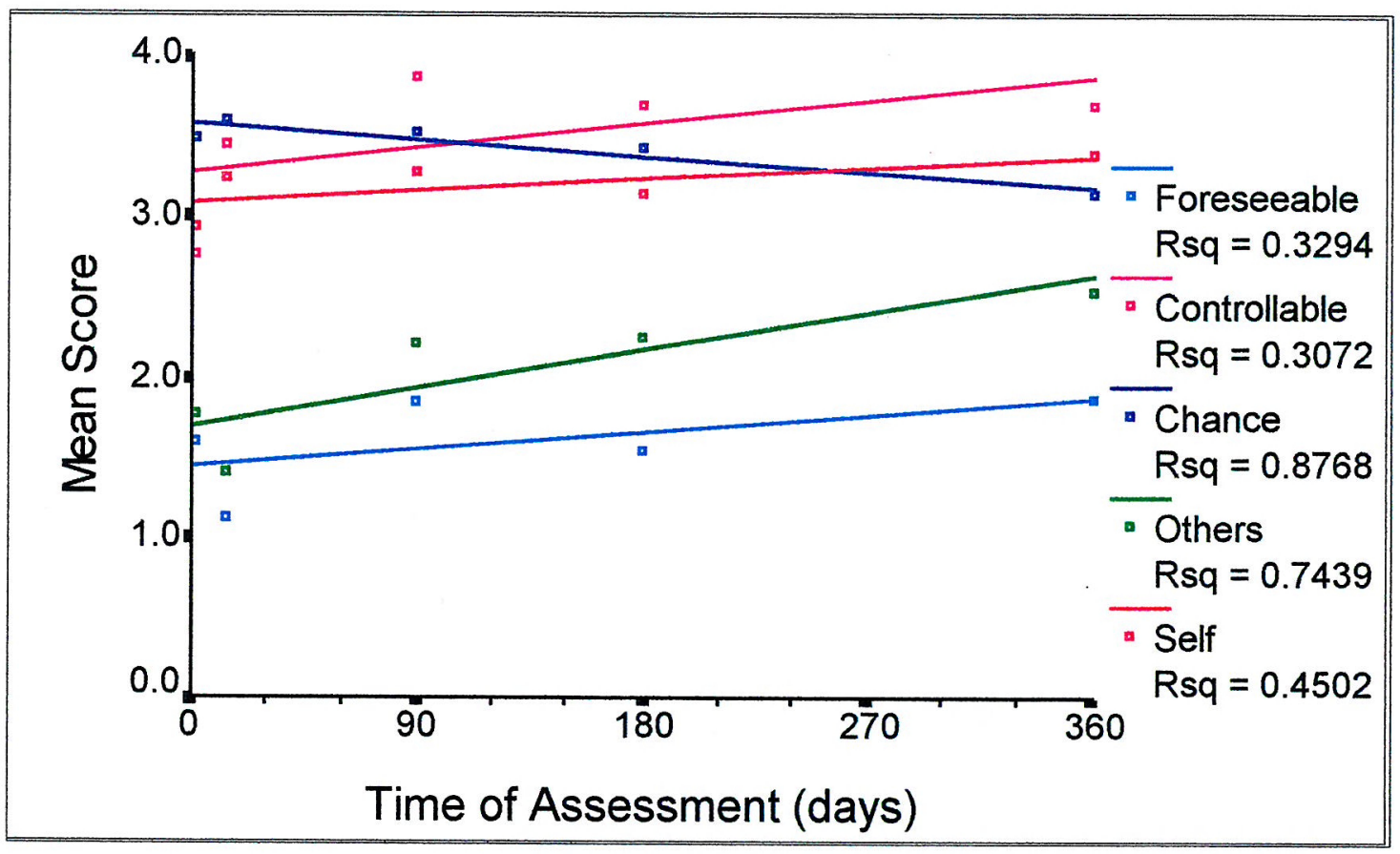

\subsection{Secondary analysis}

From the work already presented in this thesis, it is clear that control issues are complex, and can be influenced by many factors. With a project like the 'Back Home' study, it would be all too easy to analyse the data by correlating every variable with every aspect of the locus of control and perceived control outcome measures. However data dredging of this nature must be avoided, (not least because by chance alone, one result in twenty is likely to be significant).

In the primary analysis of the data, trends in control perceptions were considered over time. However it was possible that other factors may have influenced these perceptions, 
including: clients' attitudes about the cause of their back pain; their anxiety levels; and their satisfaction with the management of their back pain episode. Therefore it was necessary to undertake further analyses to address the questions of how these three factors influence clients' perceptions of control over time? It is recognized that with three factors and five assessments, the analysis risked a significant finding by chance alone. (This is why these results do not form part of the main analysis - although it would be inappropriate to omit this part of the analysis altogether and thus ignore any influence of these three factors.) Each of these three factors is now considered individually in sections $7.5-7.7$. The rationale for any statistical tests undertaken is addressed in the relevant section of this chapter.

\subsection{Influence of attitude on control}

The first factor to be analysed that potentially may influence perceptions of control, was clients' attitude towards their back pain. Theoretically it was possible that clients who attributed the cause of their back pain to chance, or other people / circumstances, may have felt less empowered to control their symptoms by their own actions, than clients who attributed the cause of their back pain to their own behaviour. This would have been an interesting question to address. However, from the (quantitative) design of the 'Back Home' study, it was not possible to gain a deep understanding of clients' attitudes about the onset of their pain. Nevertheless, in assessment two, completed at two weeks, one outcome measure was used (attitude A), which aimed to establish clients' (unprompted) perceptions of what caused their episode of back pain.

\subsubsection{Attitude measurement}

In trying to establish clients' perceptions of the cause of their symptoms, the instrument asked one open question: 'What do you think caused your back pain? Please list all the possible factors that may have contributed'. Clients then had a large space in which to respond. Once they had completed this, the second part of the instrument then asked clients to select any contributory factors from a list - see appendix 5.11 - based on the clinical experience of the research team and the responses from clients in the pilot study (described in chapter five). It was the unprompted responses that were pertinent to this thesis and these are now discussed. 


\subsubsection{Analysis of attitude responses}

The responses from 58 clients were analysed by identifying categories and recording the frequency of clients who identified the category as a causative factor.

In order to ascertain reliability for this analysis, field notes made by the researcher were checked (see section 5.7) and the data were given to an independent, impartial researcher who had not been involved with the data collection, who coded the data (blind), and the results of the two analyses were compared.

From the coding, there were eight differences in the categories out of the 119 comments identified $(7 \%)$. These differences were resolved by a third review of the data (undertaken jointly by the researcher and independent analyst), and these discrepancies were readily negotiated. The 119 comments were assigned to 28 categories, which are presented in table 7.5 .

\subsubsection{Discussion of the results}

It is interesting to note that only six categories were common to five or more clients, suggesting that either the aetiology of low back pain is multi-factorial, or our coded categories were too specific. It is likely that both issues are pertinent, but it is easier to amalgamate categories into broader groups at a later stage if they are deemed too specific, than have to revisit the raw data to isolate more specific categories.

Overwhelmingly, at assessment two (completed at two weeks), manual handling was perceived by clients to be the most likely causative factor for the onset of their acute low back pain in this study. With hindsight, it would have been interesting to ask this same question at the subsequent assessments (at three, six and twelve months), to see whether clients' perceptions were consistent, or whether they changed over time.

Again, it is evident from table 7.5 that the vast majority of factors identified are activityrelated as opposed to pathological (e.g. wear and tear, hormones) or exogenous (e.g. cold). This would suggest that clients have some degree of control over these activities, although it is not possible to gain an accurate insight into the 18 clients who attributed their symptoms to work - it may be that they can take responsibility for their tasks and working postures, or they may be set by external sources, such as conveyor belts, for example. 


\section{Table 7.5 Clients perceptions of the cause of their back pain}

Results from the 'attitude' outcome measure which asked clients to list all the possible factors which they thought caused their back pain. 119 comments were coded from the responses of 58 clients.

\begin{tabular}{|c|c|c|}
\hline Rank & Category & Frequency \\
\hline 1 & Lifting / manual handling & 31 \\
\hline 2 & Work & 18 \\
\hline 3 & Don't know & 12 \\
\hline 4 & Bending & 11 \\
\hline 5 & Trauma & 7 \\
\hline 6 & Driving & 5 \\
\hline 7 & Sitting & 4 \\
\hline 8 & $\begin{array}{l}\text { Housework / chores } \\
\text { Getting in / out of vehicle } \\
\text { Pulling } \\
\text { Twisting }\end{array}$ & 3 \\
\hline 12 & $\begin{array}{l}\text { Standing } \\
\text { Gardening }\end{array}$ & 2 \\
\hline 14 & $\begin{array}{l}\text { Walking } \\
\text { Previous history } \\
\text { Carrying child } \\
\text { Gym } \\
\text { Wear and tear } \\
\text { Sleeping position } \\
\text { Stress } \\
\text { Hormone-related } \\
\text { Opening doors } \\
\text { Tiredness } \\
\text { Riding a motorcycle } \\
\text { Driving a left-hand drive car } \\
\text { Cold } \\
\text { Playing cricket } \\
\text { Bad luck }\end{array}$ & 1 \\
\hline
\end{tabular}


Interestingly few lifestyle factors were identified (e.g. smoking, sporting activities or hobbies). Such factors are likely to be enjoyed by individuals as they have chosen to undertake them, whereas it may be that the factors identified in table 7.5 are perceived as tasks which have to be undertaken by clients, and therefore not enjoyed to the same extent. This raises issues such as whether clients are posturally tense when they are undertaking activities that they have to do, or could it be interpreted that they are more likely to 'blame' something that they don't like doing for any adverse effects? (This process may not even be at a conscious level.)

From the results, twelve clients $(21 \%)$ did not know what caused their back pain, and anecdotally, during the home visits, many of these clients expressed considerable frustration at not identifying a cause for their symptoms. For example one client said:

'My doctor told me to be careful - but how do I know what to be careful about as I don't know what set all this off in the first place. It's so annoying.'

Another client commented:

'It's not fair.... How can I be in this much pain when nothin' happened to cause it?'

The cause of this episode of back pain was something that all clients (without exception) mentioned at the first home visit, and the field notes taken at that time were checked against the results of the attitude outcome measure. In their verbal accounts, clients often described in great detail the events leading up to the onset of symptoms. Therefore, it was important to see whether their perceptions of the cause of pain affected their locus of control responses on the M.H.L.C. (form C).

\subsubsection{Effect of attitude on perceptions of control}

Due to the brevity of data collected with the attitude outcome measure, only the simplest analysis could be undertaken. For example, if clients had identified causative factors as 'work' or 'lifting', from this information alone, it was impossible to determine whether they perceived the contributing factors to be something about themselves, other people or circumstances, or chance. 
Because of the ambiguity of the data collected, from table 7.5, the only factor (recorded by more than one client) which could be used in this analysis was the 'don't know' category, identified by twelve clients.

It was expected that these twelve clients who did not have any idea what factors contributed to their pain, would score higher on the M.H.L.C. chance subscale than the 46 clients who were able to identify a cause.

Using non-parametric statistics, when the M.H.L.C. (form C) data were analysed, no such trend was evident. Thus in this population, whether or not clients were able to identify a cause for their low back pain did not appear to influence their locus of control perceptions, when measured by the M.H.L.C. (form C).

\subsection{Influence of anxiety on control}

Another factor that could theoretically influence clients' perceptions of control, was anxiety. Therefore, this has been included in the secondary analysis. It would be logical to assume that clients who were experiencing greater levels of anxiety, would feel less able to manage their symptoms, and thus would score lower on the internal subscale of the M.H.L.C. (form C) instrument. However, to date no studies have been published which address this issue in a population of clients with acute low back pain and this relevant, interesting question could readily be answered as part of the secondary analysis.

As discussed in chapter four, the instrument selected to measure anxiety in this thesis (the 6-item short form of the State scale of the Spielberger State-Trait Anxiety Inventory ${ }^{(6)}$ ), did not live up to our expectations, especially as a number of clients initially completed it incorrectly, and the data required careful scrutiny at the time of collection. However, despite the limitations of the outcome measure, the potential importance of anxiety on issues of control warrants some analysis.

\subsubsection{Changes in clients' levels of anxiety over time}

Before any effect of anxiety on clients' perceptions of control could be determined, it was necessary to establish how the results of the anxiety outcome measure changed over time in this population. It must be remembered from chapter six, that in the majority of cases, clients' acute low back pain had either resolved or significantly improved by 
assessment three (at three months). Thus, it might be expected that clients' anxiety levels would also be decreasing at a similar rate (between assessments one and three), as their symptoms resolved. In addition, some clients had received a copy of the 'Back Home' leaflet, which may have influenced their state of anxiety, and so this factor must also be considered in the analysis. Therefore, the anxiety levels of clients over time, with and without the leaflet are presented in table 7.6.

\section{Table 7.6 Results of anxiety scores over time}

The table shows the results of the mean scores from the 6-item short form of the State scale of the Spielberger State-Trait Anxiety Inventory ${ }^{(6)}$ for clients participating in the 'Back Home' study. $\mathrm{C}=$ control group (i.e. no leaflet), $\mathrm{L}=$ leaflet. The possible score range with this instrument $=6-24$ : the higher the score, the greater the level of anxiety.

\begin{tabular}{||l|cc|cc|cc|cc|cc||}
\hline \hline Assessment & \multicolumn{2}{|c|}{1} & \multicolumn{2}{|c|}{2} & \multicolumn{2}{c|}{3} & \multicolumn{2}{c|}{4} & \multicolumn{2}{|c|}{5} \\
& $\mathrm{n}=63$ & \multicolumn{2}{|c|}{$\mathrm{n}=58$} & $\mathrm{n}=57$ & $\mathrm{n}=49$ & $\mathrm{n}=47$ \\
\hline Client group & $\mathrm{C}$ & $\mathrm{L}$ & $\mathrm{C}$ & $\mathrm{L}$ & $\mathrm{C}$ & $\mathrm{L}$ & $\mathrm{C}$ & $\mathrm{L}$ & $\mathrm{C}$ & $\mathrm{L}$ \\
\hline Anxiety level & 12.2 & 12.0 & 11.3 & 10.6 & 9.9 & 8.6 & 8.7 & 8.7 & 9.5 & 8.9 \\
\hline
\end{tabular}

Not surprisingly, the results show a general trend of anxiety scores decreasing over time i.e. as the majority of clients' symptoms resolve. The results of the combined leaflet and control groups are shown as a box-and-whisker plot in figure 7.7, which shows this trend, as the median decreases with time.

The (unusual) lack of lower whiskers on the plots of assessments two, three, four and five arises because of the scoring system for this anxiety outcome measure. If clients perceive no anxiety at all (or equivalent) in the six statements, they received a score of one for each item. Therefore, the minimum score achievable with this instrument is six, out of a maximum of 24. Since a score of six also marks the $25^{\text {th }}$ percentile, there is no lower whisker on the box-plot. Such a flooring effect may bring into question the appropriateness of a box-and whisker plot, however, in such a form, the results can be readily compared to others in this chapter. 


\section{Figure 7.7: Mean anxiety scores over time}

The chart shows the anxiety scores from assessments $1,2,3,4$ and 5, measured with the 6item short form of the State scale of the Spielberger State-Trait Anxiety Inventory ${ }^{(6)}$.

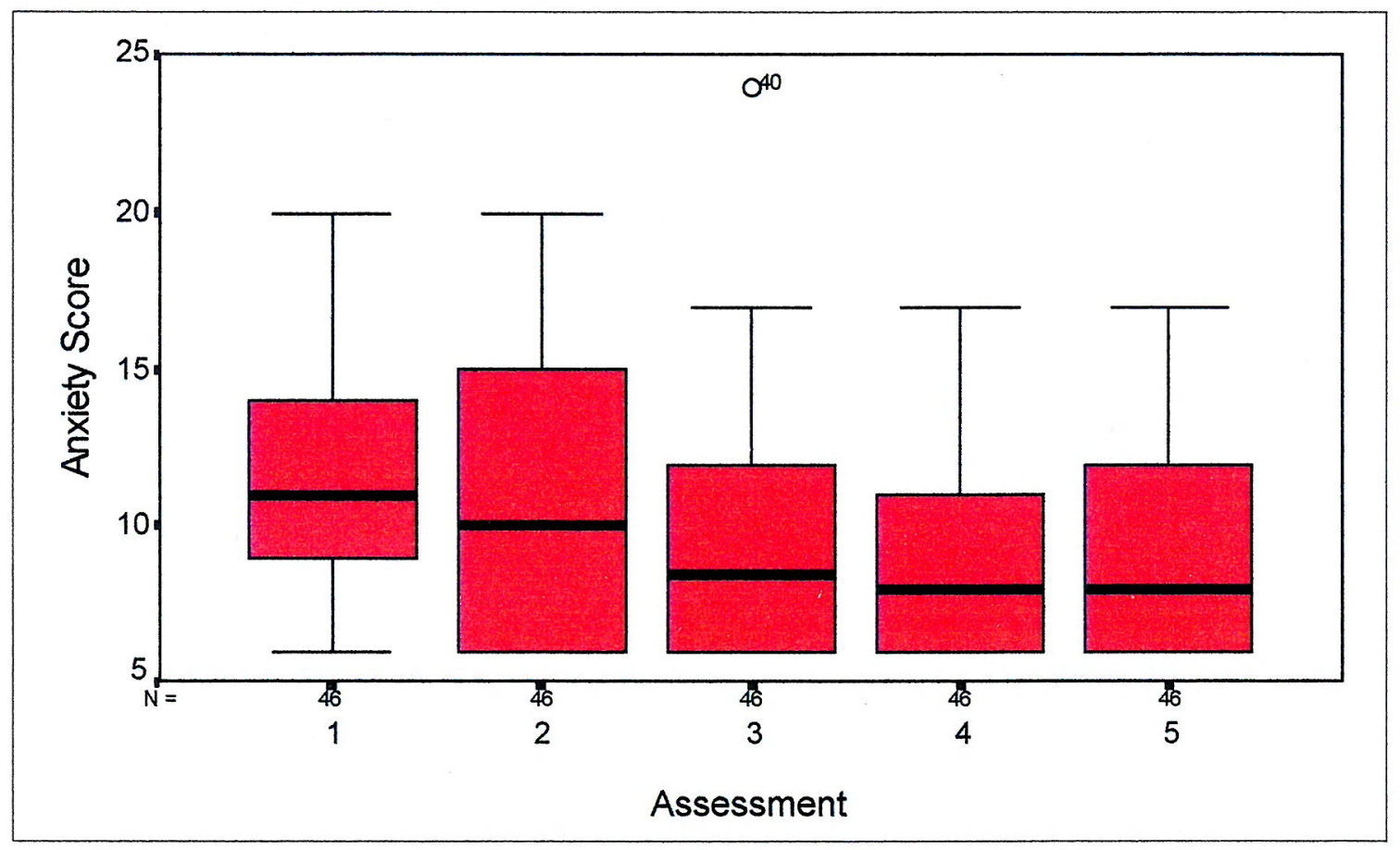

\subsubsection{The effect of the 'Back Home' leaflet on clients' levels of anxiety}

Having considered the results of the client group as a whole, it was necessary to see whether there were any differences in anxiety between the leaflet and control group. When this analysis was done, at every assessment, the control group (who did not receive the leaflet) reported higher levels of anxiety than clients who had had received the leaflet (with the exception of assessment four, where the results were identical). Despite this trend, no statistically significant differences were seen between the two groups. It might have been assumed that the greatest difference between the two groups was likely to be seen at assessment one, although even here, the difference between the groups was minimal (Mann-Whitney $=0.851$ ). This suggests that the 'Back Home' leaflet did not affect clients' levels of anxiety when measured with the 6-item short form of the State scale of the Spielberger State-Trait Anxiety Inventory ${ }^{(6)}$, or the instrument lacked the sensitivity to detect any change: The former is more likely. 


\subsubsection{Association between clients' anxiety and their perceptions of control}

Having considered how clients' anxiety levels change over time and the influence of the 'Back Home' leaflet, the final part of this anxiety analysis was to explore whether any association exists between clients' anxiety levels and their perceptions of control.

Therefore a simple analysis was performed using Spearman's Correlation Coefficient, where the client group were considered as a whole (irrespective of whether or not they had received a leaflet). From the results of the previous section, there was no need to control for the leaflet in the analysis as it had so little influence on clients' reported anxiety levels. Spearman's Correlation Coefficient (a non-parametric test) was used because the data from the 6-item short form of the State scale of the Spielberger StateTrait Anxiety Inventory ${ }^{(6)}$ were ordinal, as was the M.H.L.C. (form C). The results are summarized in table 7.7 .

\section{Table 7.7. The association between anxiety and perceptions of control}

The table shows the results of the correlations between the 6-item short form of the State scale of the Spielberger State-Trait Anxiety Inventory ${ }^{(6)}$ and the M.H.L.C. (form C), collected at assessments $1,3,4$ and $5 .{ }^{*}=$ significant at the 0.05 level.

\begin{tabular}{|l|c|c|c|c|}
\hline & $\begin{array}{c}\text { Assessment 1 } \\
\mathrm{n}=60\end{array}$ & $\begin{array}{c}\text { Assessment 3 } \\
\mathrm{n}=56\end{array}$ & $\begin{array}{c}\text { Assessment 4 } \\
\mathrm{n}=48\end{array}$ & $\begin{array}{c}\text { Assessment 5 } \\
\mathrm{n}=47\end{array}$ \\
\hline Internal & 0.473 & 0.079 & 0.359 & 0.322 \\
\hline Powerful others (doctors) & 0.788 & 0.440 & $0.019^{*}$ & 0.912 \\
Powerful others (others) & 0.298 & 0.347 & $0.018^{*}$ & 0.508 \\
\hline Chance & 0.269 & 0.658 & 0.221 & $0.047^{*}$ \\
\hline
\end{tabular}




\section{Figure 7.8: Correlation of anxiety and perceptions of control}

The two plots show the correlations of anxiety and external locus of control, subdivided into powerful others (doctors) and (others), from assessment four (completed at six months).
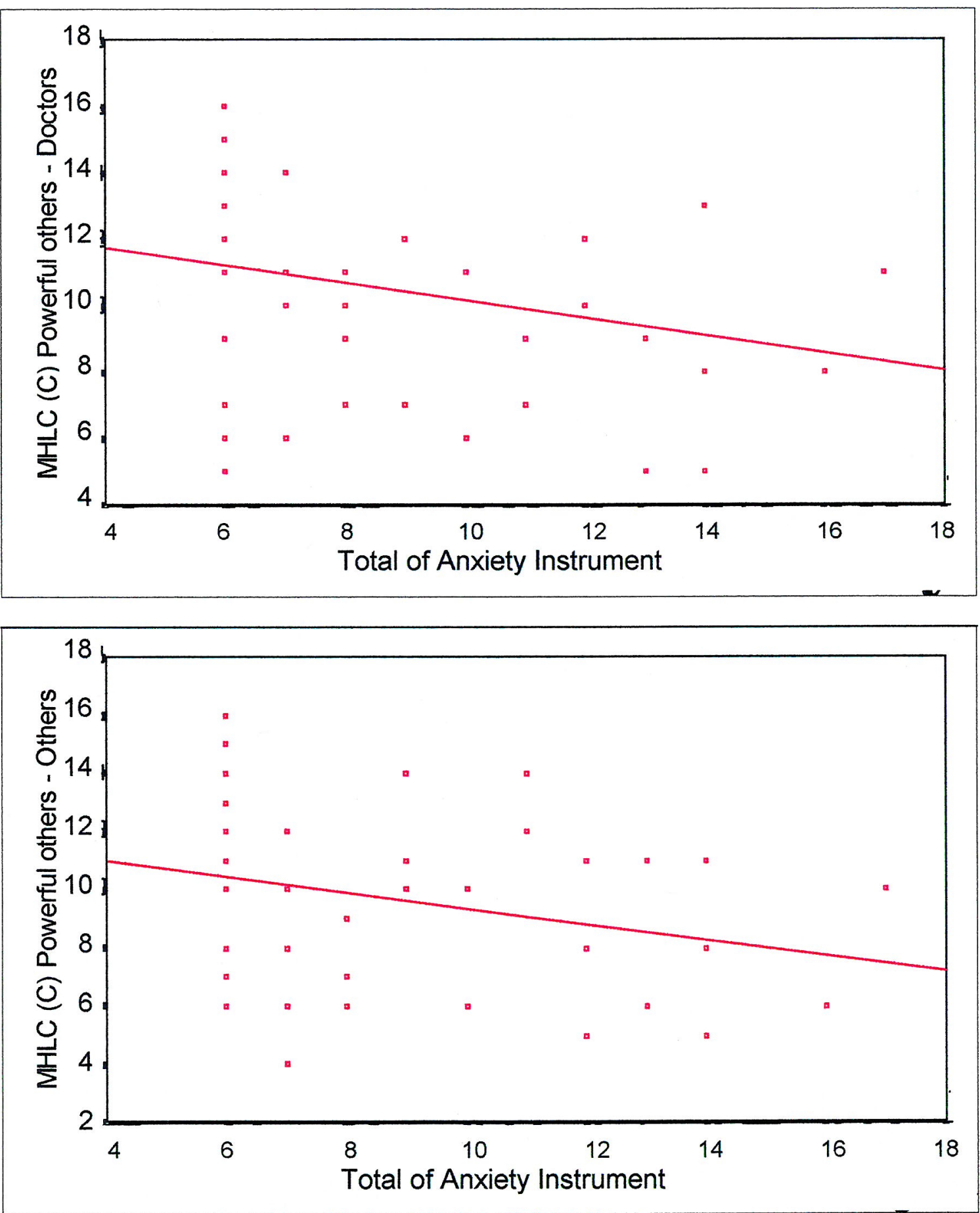
The only notable findings from table 7.7 were the powerful others external subscales (doctors and others) in assessment four. On closer inspection of this result, when the data are plotted (see figure 7.8), it is clear that anxiety and perceptions of control on the external powerful others subscale, are negatively correlated. It is unclear why this is a stronger association at six months than at any other time in the analysis, and it must be remembered that the sample size decreased from 56 in assessment three, to 48 in assessment four, for these outcome measures. (The reasons for this difference are discussed in section 6.3.3.)

\subsection{Satisfaction}

Having considered that clients' attitude and their levels of anxiety may influence their perceptions of control, it should also be considered that clients' satisfaction with the information they had received and the way their back pain episode was being managed, could have affected their perceptions of control.

This was demonstrated in the 'Back Home' study when one client experienced an adverse reaction to the analgesic medication (dihydrocodeine), prescribed by his G.P. This experience appeared to over-shadow all other input given by the G.P., and thereafter, the client returned a score of zero when asked to rate his satisfaction with the amount and content of information given, stating that his G.P. had not warned him about the tablets. He then reasoned:

'My back is only better 'cos of my treatment - exercises... Not those bloody useless tablets, which I chucked away'.

In this particular instance, this unfortunate reaction clearly affected the client's perceptions of control over his symptoms and thus the influence of satisfaction on control warrants further exploration.

Perhaps it might be logical to assume that clients who felt dissatisfied with the information they had received for this episode of back pain, would be likely to show less internality than clients who were more satisfied with the information they received. However, such a notion assumes that dissatisfied clients wanted more information than they claimed to have received. In practise, it might be that they received more 
information that they actually wanted, which had been the cause of their dissatisfaction (although this seems unlikely, given the findings of the G.P. questionnaire in chapter three).

\subsubsection{Changes in clients' satisfaction levels over time}

Once again, before any influence of clients' satisfaction on their perceptions of control could be established, it was necessary to determine how satisfaction levels vary over time. All the assessments included three questions about clients' satisfaction with the amount, and content of the information they had received, and the way their back pain was being managed. (Assessment one also included an additional question about clients' satisfaction with the visit to their G.P.) These questions were $10 \mathrm{~cm}$ visual analogue scales (V.A.S.), with end points of 'totally dissatisfied' and 'totally satisfied' (see appendix 5.12). As these questions all assessed facets of the G.Ps' response to their client's back pain, the results of the three V.A.S. questions were summated and are presented as a 'total' score in table 7.8 and graphically in figure 7.9, which shows clients' satisfaction scores over time.

\section{Table 7.8: Clients' satisfaction scores over time}

The table shows the mean results of clients' satisfaction with the amount, and content of information they received and the way their back pain was being managed, recorded by summation of the three V.A.S. The minimum score $=0$ and the maximum $=300$ : the higher the score, the greater the level of satisfaction.

\begin{tabular}{|l|c|c|c|c|c|}
\hline Assessment & $\begin{array}{c}1 \\
\mathrm{n}=63\end{array}$ & $\begin{array}{c}2 \\
\mathrm{n}=58\end{array}$ & $\begin{array}{c}3 \\
\mathrm{n}=57\end{array}$ & $\begin{array}{c}4 \\
\mathrm{n}=49\end{array}$ & $\begin{array}{c}5 \\
\mathrm{n}=48\end{array}$ \\
\hline $\begin{array}{l}\text { Satisfaction } \\
\text { score }\end{array}$ & 177.0 & 182.8 & 203.1 & 207.7 & 200.1 \\
\hline
\end{tabular}




\section{Figure 7.9: Mean satisfaction scores over time}

The chart shows the satisfaction scores from assessments 1,2,3,4 and 5, measured with three V.A.S. combined: a) satisfaction with the amount of information received; b) with the content of information received; and c) with the way the episode of back pain was being managed.

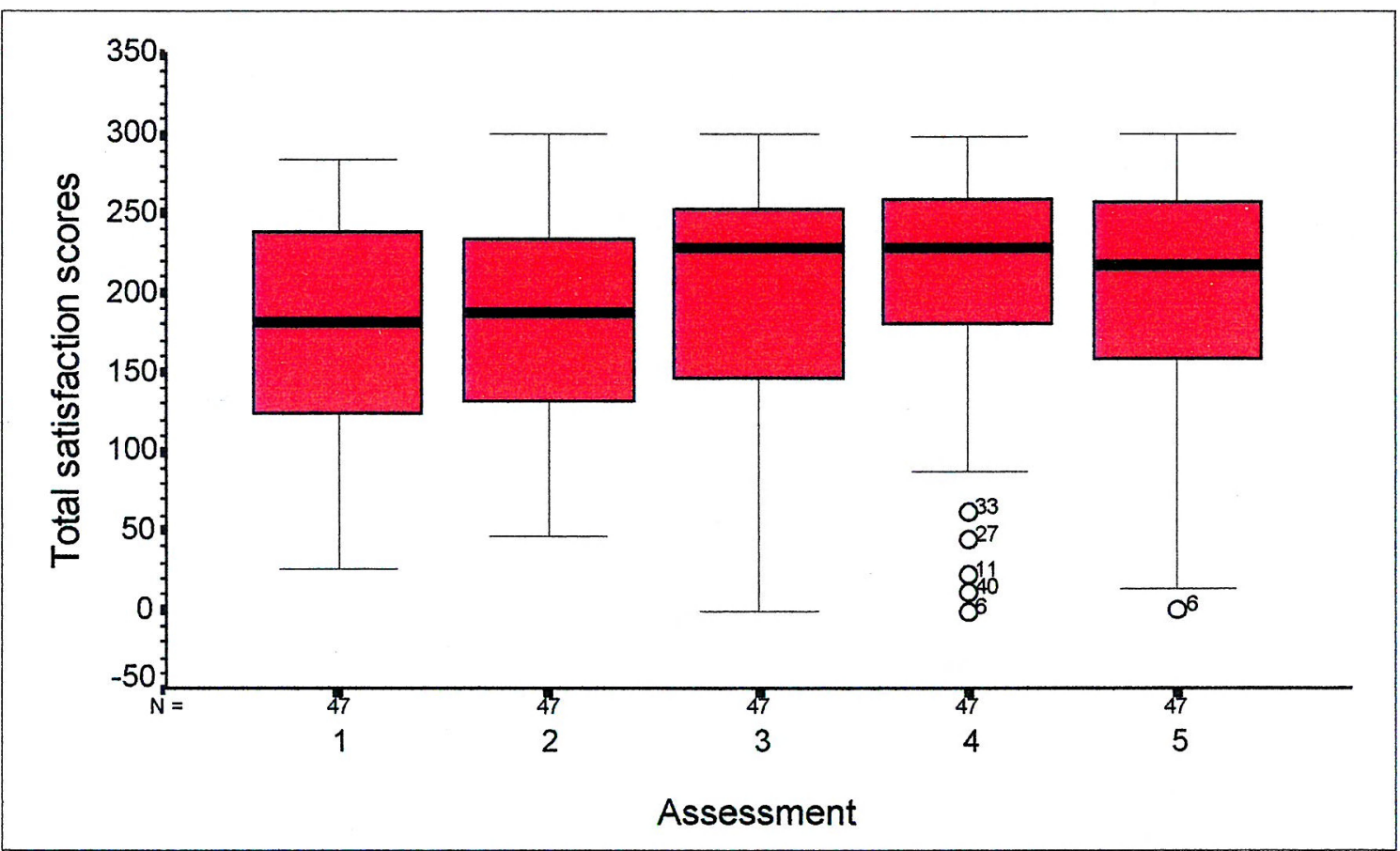

From this data, it can be seen that generally clients' satisfaction levels increased over time, presumably influenced by the resolution of their symptoms (in most cases).

It is worth noting that many biases exist in clients' perceptions of satisfaction. To illustrate a positive bias, one client commented whilst completing question three of the satisfaction instrument (during assessment two):

'My G.P. is good, and especially with you coming and being interested in my back'.

Therefore the client had not been able to isolate the G.P's role in their back pain episode from the research study (which was not unreasonable as he perceived it all as part of the way his back pain episode was being managed).

There were also some negative biases in the data collection. For example, the client who was prescribed dihydrocodeine tablets for pain relief, cited earlier in this section. 


\subsubsection{The effect of the 'Back Home' leaflet on clients' satisfaction}

As before, there was the added dimension of some clients having received the leaflet, which needed to be addressed in the analysis. Therefore, clients' satisfaction scores, with and without the leaflet are summarized in table 7.9.

\section{Table 7.9: Results of satisfaction scores over time}

The table shows the results of the mean scores from the visual analogue satisfaction scales for clients participating in the 'Back Home' study. $\mathrm{C}=$ control group (i.e. no leaflet), $\mathrm{L}=$ leaflet. The possible score range $=0-100$ : the higher the score, the greater the level of satisfaction. The questions asked 'How satisfied are you with ....

Visit to G.P. $\quad$ = '... your visit to your doctor for your back pain?'

Amount of information $\quad=$ '... with the amount of information you received for your back pain?'

Content of information $\quad=$ '... with the content of information you received for your back pain?'

Back pain management = '... the way your back pain is being managed?'

\begin{tabular}{||l|cc|cc|cc|cc|cc||}
\hline & \multicolumn{2}{|c|}{$\begin{array}{c}\text { Assessment 1 } \\
\mathrm{n}=63\end{array}$} & \multicolumn{2}{|c|}{$\begin{array}{c}\text { Assessment 2 } \\
\mathrm{n}=58\end{array}$} & \multicolumn{2}{|c|}{$\begin{array}{c}\text { Assessment 3 } \\
\mathrm{n}=57\end{array}$} & \multicolumn{2}{|c|}{$\begin{array}{c}\text { Assessment 4 } \\
\mathrm{n}=49\end{array}$} & \multicolumn{2}{|c|}{$\begin{array}{c}\text { Assessment 5 } \\
\mathrm{n}=48\end{array}$} \\
\hline & $\mathrm{C}$ & $\mathrm{L}$ & $\mathrm{C}$ & $\mathrm{L}$ & $\mathrm{C}$ & $\mathrm{L}$ & $\mathrm{C}$ & $\mathrm{L}$ & $\mathrm{C}$ & $\mathrm{L}$ \\
\hline Visit to G.P. & 67.9 & 73.5 & - & - & - & - & - & - & - & - \\
\hline $\begin{array}{l}\text { Amount of } \\
\text { information }\end{array}$ & 55.3 & 63.9 & 55.8 & 62.7 & 61.8 & 71.1 & 62.4 & 74.0 & 61.0 & 70.6 \\
\hline $\begin{array}{l}\text { Content of } \\
\text { information }\end{array}$ & 51.5 & 61.9 & 57.0 & 60.5 & 60.2 & 70.4 & 58.0 & 78.1 & 61.0 & 69.2 \\
\hline $\begin{array}{l}\text { Back pain } \\
\text { management }\end{array}$ & 52.9 & 65.0 & 62.4 & 65.8 & 68.9 & 71.2 & 63.8 & 76.3 & 66.1 & 72.3 \\
\hline Total (\%) & 159.7 & 190.9 & 175.2 & 189.1 & 190.9 & 212.7 & 184.3 & 228.4 & 188.2 & 212.1 \\
\hline
\end{tabular}

* Excluding results of clients' satisfaction with G.P. visit, as this was only asked in assessment one.

From these results, it is clear that in every case, the leaflet group had higher levels of satisfaction than the control group (who had not received the 'Back Home' leaflet). In order to evaluate the size of these differences, the data were analysed using an unrelated t-test (parametric), as the V.A.S. data were taken from two, different, unmatched subject $\operatorname{groups}^{(7)}$.

This judgement was made on the understanding that the data obtained from V.A.S. are considered interval / ratio (although it is recognized that this view is not universal, since 
some may consider such data to be ordinal). However in this instance, with assumed, interval / ratio data taken from two, different, unmatched subject groups, this fitted the criteria for using an unrelated t-test ${ }^{(7)}$.

From the 21 results analysed, only one (question 2 in assessment 4 i.e. 'How satisfied are you with the content of information you have received for your back pain?'), was significant $(\mathrm{t}=-2.50, \mathrm{df}=38, \mathrm{P}=0.017)$. However, little importance is attached to this finding, as by chance it is likely that one result in twenty will be significant when this volume of data is analysed. This analysis was looking for trends of satisfaction with time, and no conclusive evidence was found. Therefore in this study, clients who had received the 'Back Home' leaflet showed higher levels of satisfaction with the amount and content of the information they received and the way their back pain was being managed, than clients who did not receive the leaflet. These differences however, were not statistically significant. It may also be pertinent to consider that by G.Ps giving out a leaflet, they may be showing extra interest in their clients, which may contribute to higher levels of satisfaction in these clients.

\subsubsection{Association between clients' satisfaction and their perceptions of control}

Having considered how clients' satisfaction levels change over time and the influence of the 'Back Home' leaflet, in the same format as the anxiety section, the final part of this analysis was to explore whether any association exists between clients' satisfaction levels and their perceptions of control.

Once again, a simple analysis was performed using Spearman's Correlation Coefficient, as although the V.A.S. scores were interval / ratio, the M.H.L.C. (form C) was only ordinal and hence non-parametric statistics were appropriate. As before, the client group were considered as a whole (irrespective of whether or not they had received a leaflet, as there was no need to control for this variable), and the results are summarized in table 7.10 . 


\section{Table 7.10. Association between satisfaction and perceptions of control}

The table shows the results of the correlations between the V.A.S. measuring clients levels of satisfaction (with the amount and content of information they received, and the way their back pain episode was being managed), and the M.H.L.C. (form C), collected at assessments $1,3,4$ and $5 .^{*}=$ significant at the 0.05 level.

\begin{tabular}{|l|c|c|c|c||}
\hline & Assessment 1 & Assessment 3 & Assessment 4 & Assessment 5 \\
\hline Internal & 0.978 & 0.396 & 0.922 & 0.631 \\
\hline Powerful others (doctors) & 0.855 & 0.072 & $0.000^{*}$ & 0.442 \\
Powerful others (others) & 0.079 & 0.619 & 0.153 & 0.481 \\
\hline Chance & 0.595 & 0.750 & 0.212 & 0.935 \\
\hline
\end{tabular}

Similar to the anxiety results, the external powerful others subscales in assessment four warrant further investigation, and are therefore plotted in figure 7.10. From this plot, it is clear that satisfaction and perceptions of control (external powerful others subscale) are positively correlated. As before, it is unclear why this is a stronger association at six months than at any other time in the analysis. Again, little importance is attached to the significant finding due to the volume of data analysed. 
Figure 7.10: Correlation of satisfaction and perceptions of control

The two plots show the correlations of satisfaction and external locus of control, subdivided into powerful others (doctors) and (others), from assessment four (completed at six months).
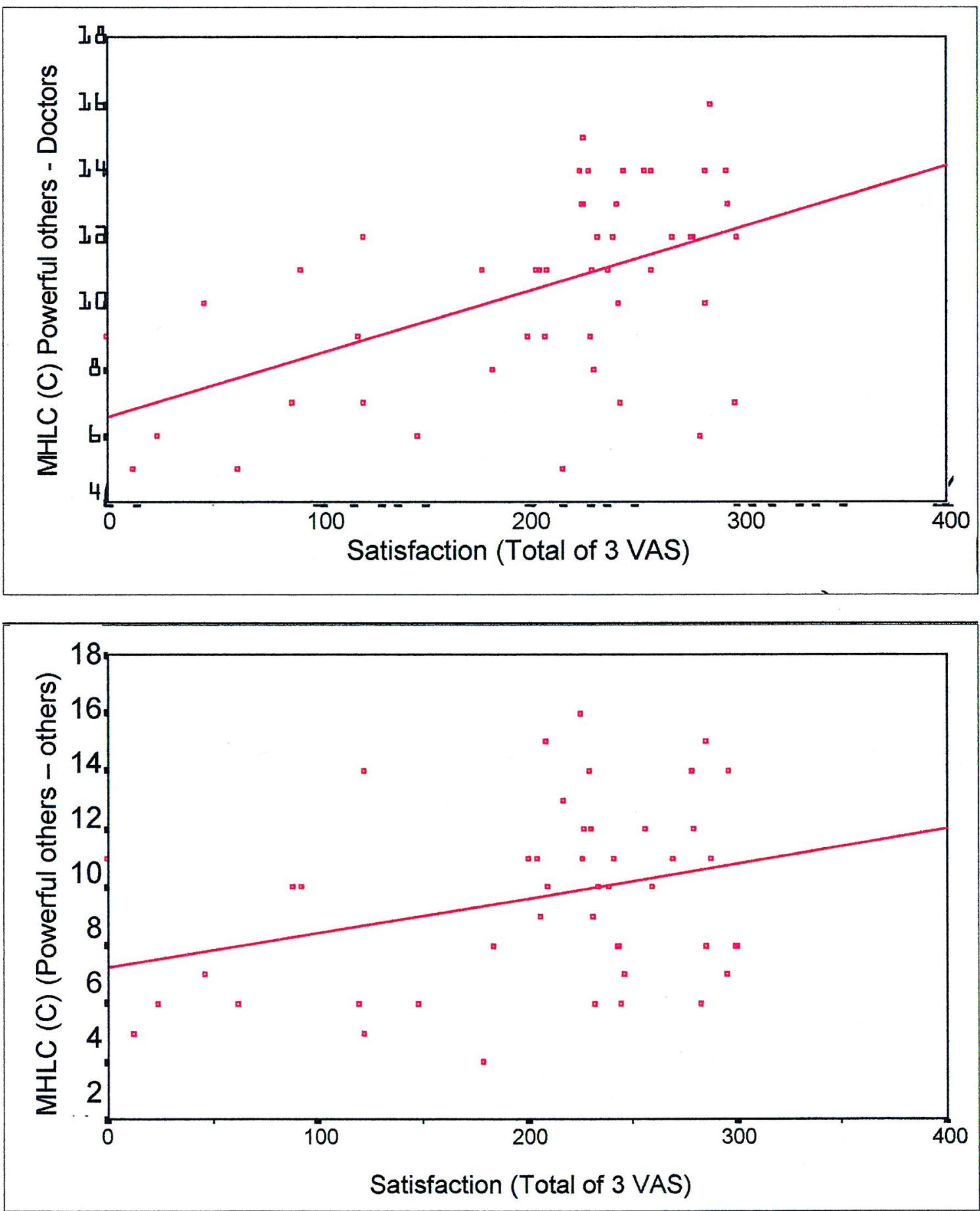


\subsection{Summary of the secondary analyses}

This chapter specifically addresses control issues in clients with acute low back pain, and the primary analysis focuses on changes in clients' perceptions of control over time. However, little is reported in the literature on control issues in a population of people with acute back pain and therefore it was necessary to examine the data further, looking for trends which might indicate how clients' attitudes, levels of anxiety and satisfaction influence their perceptions of control.

Since the analysis needed to consider time, any influence arising from the 'Back Home' leaflet and the resultant effect on clients' perceptions of control, the analysis was vast, and the risk of data dredging, high. This is why some apparently significant findings have been discounted. This secondary analysis was undertaken specifically to identify trends in the control literature, which might be either clinically significant or useful in subsequent studies - it was not a quest for statistical significance.

From the work in sections $7.5-7.7$, eight main points arise:

- Many factors are perceived by clients to contribute to the aetiology of their back pain. In this sample of 58 clients with acute low back pain, at two weeks, the most likely causative factor they identified for the onset of their symptoms was manual handling.

- The majority of causative factors identified by clients were activity-related, in this sample.

- Whether or not clients were able to identify a cause for their low back pain did not appear to influence their locus of control perceptions when measured by the M.H.L.C. (form C).

- In general, clients' anxiety levels decreased with time in this study.

- Clients who received a copy of the 'Back Home' leaflet reported lower levels of anxiety than those in the control group, but these differences were not statistically significant. 
- Clients reported satisfaction with the amount and content of the information they received and the way their back pain episode was being managed generally increased over time.

- Clients who received a copy of the 'Back Home' leaflet reported (nonsignificant) higher levels of satisfaction with these information and management issues than those in the control group, who had not received a leaflet.

- When undertaking analyses with multiple variables, there is an inherent risk of 'data dredging', where approximately one result in twenty will be statistically significant by chance alone.

Despite the obvious concerns expressed in this secondary analysis (which used multiple variables), the findings have revealed useful trends, which have justified this approach.

\subsection{Measuring control}

What is emerging from this chapter is that control issues cannot be studied in isolation they may be influenced by many factors including gender, perceived levels of anxiety, satisfaction, symptomatology and probably other factors not considered in this study.

It is also apparent from chapters two and four that measuring facets of acute low back pain is analogous to hitting a moving target, as the natural history of this symptom renders measuring it particularly challenging. Nevertheless, this should not be an excuse for evading this issue.

It is clear that clients' perceptions of control do change over time, and having some understanding of this may enable clinicians to focus on the most appropriate treatment and management approaches for clients with acute low back pain to ensure maximum benefit of any intervention. Clearly there is much work to be done in this area, not least in developing more robust outcome measures. However, as discussed in 2.17.1, locus of control may be part of a wider construct and this debate must be resolved before the development of robust outcome measures can take place. Only when these measures have been sufficiently tested, can interventions be targeted at the correct time in the natural history of resolution, and optimize health gain. 


\subsection{Summary of the chapter}

- Gender issues are pertinent to health-related perceptions of control. Despite obvious gender differences in clients' perceptions of control in this study, no clear trends are evident across the literature.

- Clients who had received a copy of the 'Back Home' leaflet initially perceived themselves more able to control their back pain than those who had not received the leaflet. This difference, although statistically significant at two days, was not maintained in subsequent assessments.

- The development of simple, but robust outcome measures is essential if work on control issues is to progress. 


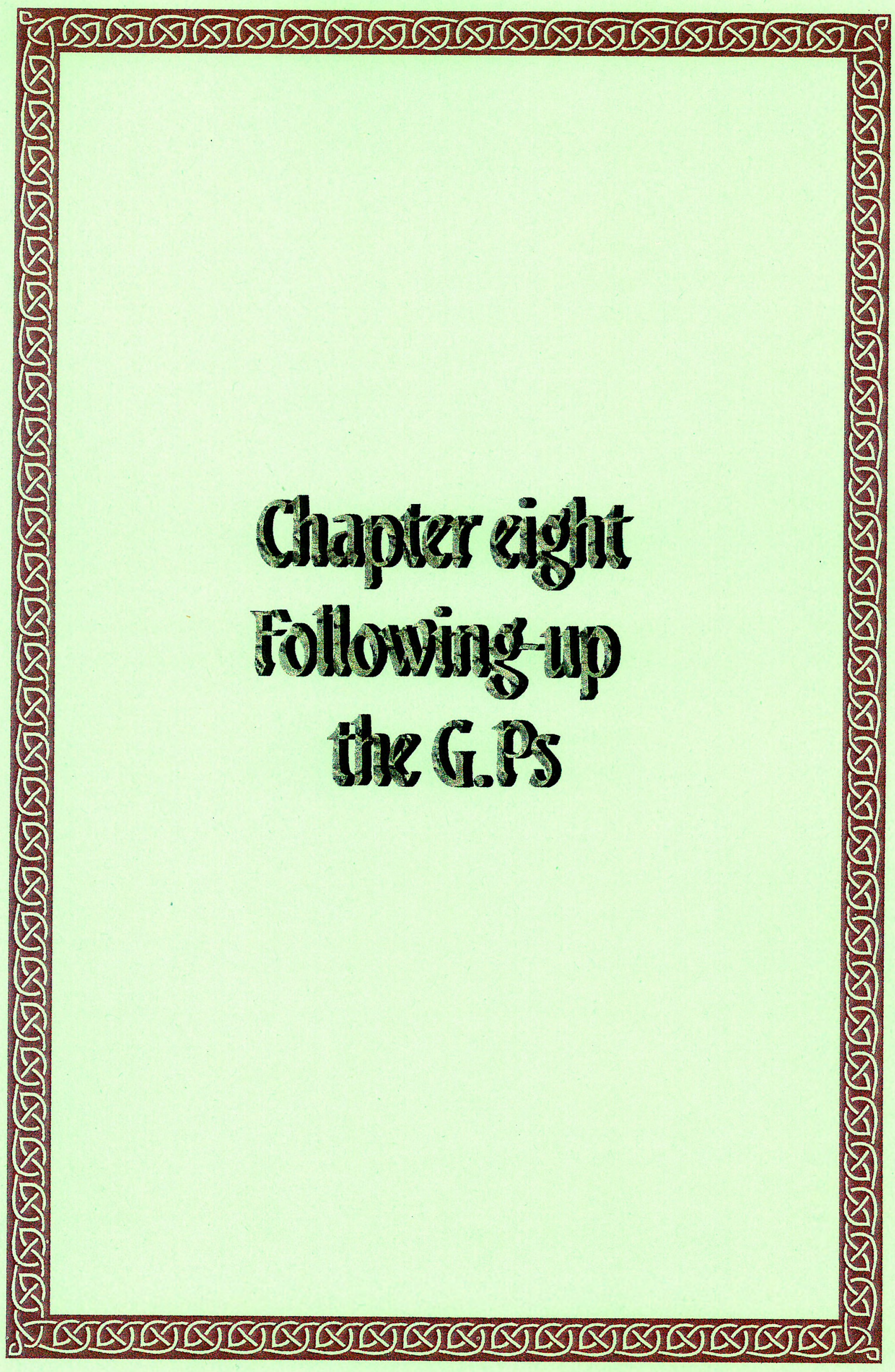




\section{Chapter eight: Following-up the G.Ps}

It can be seen from chapter five that recruiting clients into the 'Back Home' study was problematic, being considerably slower than was predicted both from the literature, and the pilot work with G.Ps described in chapter three. As a means of gaining feedback, both for the project sponsors and ourselves, we decided to address this issue directly with all the G.Ps who were registered to participate in the study.

Poor recruitment of clients had also directly affected the research of control issues and low back pain - the mainstay of this thesis, which is why it was decided to include a chapter discussing this follow-up work. This chapter also considers the implications for funding future studies with comparable study designs i.e. those requiring G.Ps to access clients from their regular surgeries.

\subsection{The size of the recruitment problem}

In section 3.1 it was estimated that G.Ps might expect to see about 300 clients each year presenting with back pain, although this estimate includes all causes of back pain (not just of musculoskeletal origin) and all durations (not just acute).

Originally, all 97 G.Ps in the New Forest area were approached and 28 agreed to participate in the study. However, after 22 weeks, only eight clients were successfully entered into the study, and so the catchment area of the study was extended Eastwards into Southampton. Although a total of 51 G.Ps consented to recruit clients for the study, between June 1994 and December 1996, only 64 clients were successfully entered from 19 of these G.Ps.

There was clearly a mismatch between the numbers of potential clients with acute low back pain that the research team had predicted from G.Ps' estimates, and the actual number they managed to recruit. Possible explanations for this mismatch might include:

- the G.Ps themselves e.g. they may not have approached all potential clients eligible to participate in the study;

- clients e.g. they may have declined to participate when asked by the G.P; 
- the study e.g. the procedure for recruiting clients into the study may be perceived as complex.

It is likely that all three aspects contributed to the poor recruitment, but the extent of each factor could not be determined accurately. On reflection, perhaps our inclusion criteria were too restrictive, especially as participating clients must have experienced no low back pain during the previous six months. However, this was to ensure that clients were considered with acute low back pain and not on-going, chronic pain.

\subsection{Following up the original G.P. questionnaire}

Originally it was intended that the questionnaire used in chapter three (establishing G.Ps' current approach to the management of clients with acute low back pain) would be repeated at the end of the study to monitor any change in clinical practise. This work may also assess the impact of clinical guidelines ${ }^{(1,2)}$, that had been published after the 'Back Home' study had started. Seeking information about both changes in clinical practise and poor client recruitment at a similar time may adversely affect response rates about the recruitment issue. Therefore, not wishing to burden the G.Ps, we prioritised and (reluctantly) omitted the questionnaire about G.Ps' current management of clients.

\subsection{Methods}

The next dilemma was deciding how best to gain information from participating G.Ps about their poor recruitment - recognizing that any requests for further information may themselves yield limited response rates.

Due to practical constraints such as geographical location of participating G.Ps, it was not feasible to organize group discussions, such as focus groups. Two methods were potentially viable - a questionnaire, or a short semi-structured interview. Theoretically, interviewing G.Ps may yield rich, thus giving a deeper understanding of the factors that adversely affected recruitment into the study. It would allow opportunities to probe answers further and enable clarification of any ambiguities or omissions in the data. 
However, there are several disadvantages:

- Time factors i.e. encroaching into G.Ps' busy schedules (especially as a few G.Ps had alluded to feeling 'over-researched' during practice visits).

- G.Ps would be offered no anonymity in an interview, and therefore may have difficulty expressing negative views in person. This could be complicated (and biased) by the researcher knowing many of the G.Ps personally, both from clinical practise and through the research.

- G.Ps who had not recruited any clients into the study may perceive a face-toface interview as confrontational. They may decline to be interviewed, which would be problematic, as these were the population we particularly wished to access.

By comparison, questionnaires may encroach less on a G.P's time than an interview or focus group, which may therefore increase response rates. Although it is claimed that questionnaires have a low cost of data collection, low cost of processing and avoid interviewer bias ${ }^{(3)}$, in our study the cost implications were not as important as the issues of burdening G.Ps by increasing their workloads.

Using a questionnaire does not eliminate all forms of bias - it would be naïve to assume this is the case. After all, it is not possible for G.Ps to recruit clients for a study without forming opinions about the study design, paperwork, and the research team. In our case, clients may also feedback directly to their G.Ps about home visits since many of them saw their G.P. on subsequent occasions e.g. for sickness certificates and further prescriptions.

When compared to interview data, the disadvantages of using questionnaires include the relative superficiality of data gained and lack of opportunity to probe any points of interest or rectify any omissions. Questionnaires have been criticized as being 'falsely prestigious because of their quantitative nature ${ }^{(4)}$, although this may depend on the design of the questions. It may be that answers generate ideas and from these, categories are formed such as the analysis of criteria for selecting x-rays (section 3.6.10). These can then be ranked, so that it is the relative importance of issues that is of interest, not the actual numbers that may be generated. 
Considering all these arguments, it was decided that on balance, the least intrusive means of accessing information about poor recruitment by G.Ps was via a brief questionnaire. In addition, rather than further burdening them with additional paperwork, it was decided to give the questionnaire a dual purpose — to also ask G.Ps whether they were interested in receiving papers or a copy of the 'Back Home' study findings. If so, they needed to complete their name and practice details. It was anticipated that few G.Ps would request these reports since they all received a copy of the abstract from the 'Back Home' study (appendix 8.1), together with a letter thanking them for participating.

Consideration had been given as to whether this question, which would breach anonymity, should have been asked on a separate piece of paper in case this limited responses where G.Ps might feel inhibited by potentially being identified. However as the prime motive was to address issues of recruitment, the number of sheets of paper and questionnaire length were of paramount importance. Yet again, there was a tension between balancing ideal and pragmatic solutions to these issues.

\subsubsection{The G.P. follow-up questionnaire}

The questionnaire consisted of only four questions, two of which related directly to the recruitment issue (see appendix 8.2). The first part was an open question asking G.Ps to list any reasons that they considered may have contributed to the slow recruitment of clients into the study. After this unprompted response, G.Ps were then asked to rate 12 listed factors to identify the extent to which they thought each factor contributed to the slow recruitment into the 'Back Home' study. These 12 factors had previously been identified by members of the research team and from discussions with G.Ps who had withdrawn from the study, citing issues that had been pertinent to their practise (see 8.3.2). G.Ps were also asked for any other comments, and finally there was the section to complete if they wanted to receive the additional reports.

\subsubsection{The participating G.Ps}

In 1997 all participating G.Ps who still remained in the study $(n=40)$ were sent a copy of the questionnaire, which was printed on 'chamois' paper. Eleven G.Ps did not complete the study for a number of reasons: leaving the practice $(n=1)$; retiring $(n=2)$; withdrawing from the study $(n=7)$; or being asked to leave the study by the research 
team due to issues of non-conformity with the study protocol $(n=1)$. The reasons cited by the seven G.Ps who withdrew from the study were: pressure of work $(n=3)$; 'the study does not really provide any help to the patients at the time when they need help' $(\mathrm{n}=2)$; unknown $(\mathrm{n}=2)$.

The demographic details of the participating G.Ps are summarized in table 8.1.

\section{Table 8.1: Demographic characteristics of the G.P. populations}

Results summarizing the demographic characteristics of the G.Ps in Southampton and the New Forest who did recruit clients into the 'Back Home' study $(n=19)$ with those who did not recruit any clients $(n=32)$ :

\begin{tabular}{|l|c|c|}
\hline & $\begin{array}{c}\text { G.Ps who did } \\
\text { recruit clients into } \\
\text { the study }\end{array}$ & $\begin{array}{c}\text { G.Ps who did not } \\
\text { recruit clients into } \\
\text { the study }\end{array}$ \\
\hline Mean number of G.Ps in the practice (total) & 5.7 & 5.7 \\
\hline Mean number of years practising as a G.P. & 11.5 & 14.9 \\
\hline $\begin{array}{l}\text { Number who have completed any post- } \\
\text { graduate courses on the management of } \\
\text { acute low back pain }\end{array}$ & $8 / 18(44 \%)$ & $10 / 30(33 \%)$ \\
\hline
\end{tabular}

There were no significant differences between the duration of experience as a G.P. and post-graduate training in acute low back pain, when comparing G.Ps who did recruit clients into the study and those who did not. Thus the reasons for variations in recruitment lie beyond demographic issues.

\subsubsection{Scoring the questionnaire}

Having considered the instrument and the population, consideration was given in advance to how the information gleaned would be analysed. The responses to the open question, asking G.Ps to identify reasons that may have contributed to the slow recruitment, were collated and the frequency of each comment was recorded.

For the list of 12 factors, a four point scale was used, with value labels: 1 = 'not at all'; $2=$ 'mildly'; 3 = 'moderately'; 4 = 'very significantly', and the G.P. rated each of the 12 responses individually, together with one additional category of 'other (please specify)'. On reflection, it would have been preferable if this four-point scale had ranged from $0-3$ as opposed to $1-4$, since factors scoring 1 were deemed not to contribute to the slow 
recruitment at all and thus should not have contributed to the score. This was an oversight, but since it was the ranking that was important (the overall score was arbitrary) and this error was consistent for all the factors listed, it was not important. Finally any other comments were noted and categorized as for the open question.

\subsection{Results of the questionnaire}

Of the 40 G.Ps who were sent a follow-up questionnaire, 24 responded (60\%), which was a higher rate than expected, considering the questionnaire addressed poor recruitment.

\subsubsection{Unprompted reasons for poor recruitment of clients}

When G.Ps were asked, to identify any reasons which they considered may have contributed to the slow recruitment of clients into the study, a total of 47 responses were obtained from the 24 G.Ps (mean $=2$ comments per G.P.) which were collated, by identifying categories from the data and recording the frequency of G.Ps who identified the category as a contributing factor. In order to ascertain reliability for this analysis, the data were given to an independent, impartial researcher, who had not been involved with the data collection, who coded the data ('blind'). The results of the two analyses were then compared. The only discrepancies (apart from subtle differences in the naming of categories), surrounded whether 'Paperwork not to hand' and 'Remembering paperwork whilst on home visit' could be considered the same category. Following further debate, it was agreed to list them separately, as it is unclear from the response whether not having the paperwork to hand occurred in the G.P. surgery, or whilst out on a home visit. A summary of the results is shown in table 8.2.

Perhaps not surprisingly, $50 \%$ of the G.Ps who responded, identified pressure of work as a factor which influenced recruitment rates of clients into the study. Examples of comments made included:

'Yet another thing for busy G.Ps to do in a pressurised surgery. The 'will' is there but in practice it is very difficult'

'Often last straw when already running late or patient brings out another 5 agendas'. 
Such comments must be considered in the context of the average surgery interval. From the G.P. questionnaire described in chapter three, the mean surgery interval $=9.1$ minutes, and the modal surgery interval $=10$ minutes. Informing a client about a research study, gaining their consent (if appropriate), and completing the necessary paperwork, all encroach on the consultation. This can add to the pressure that the G.P. is already perceiving of hosting a surgery, which presumably increases when they are behind schedule.

\section{Table 8.2: Reasons given by G.Ps for the slow recruitment of clients}

Results from an open question asking G.Ps to list any reasons that they considered may have contributed to the slow recruitment of clients into the study. The frequency column depicts the number of G.Ps who made similar comments which were analysed and subsequently grouped together.

\begin{tabular}{|c|l|c|}
\hline Rank & \multicolumn{1}{|c|}{ Reasons given } & Frequency \\
\hline 1 & Pressure of work & 12 \\
\hline 2 & Difficulty remembering & 10 \\
\hline 3 & $\begin{array}{l}\text { Other projects underway / over-researched } \\
\text { Few patients fitted the inclusion criteria }\end{array}$ & 4 \\
\hline 5 & Time consuming / process of entering patients & 3 \\
\hline 9 & $\begin{array}{l}\text { Patient not willing (to take part) } \\
\text { Paperwork not to hand } \\
\text { Poor response we get from secondary care regarding low back pain to patient } \\
\text { Areas of special interest } \\
\text { Didn't try hard enough } \\
\text { Not sure }\end{array}$ & $\begin{array}{l}\text { Remembering paperwork whilst on home visit } \\
\text { Coo early recruitment } \rightarrow \text { unnecessary intervention } \\
\text { Group most needing help = recurrent / persistent }\end{array}$ \\
\hline
\end{tabular}


Some comments related to practical issues such as:

'We are asked to find patients for lots of projects \& . would need a very large notice board over desk to have reminders for all of them'

'Now 'paperless' \& do not routinely use paper records. It would be useful if these sort of study criteria could be accessed via computer which we all use routinely'.

This is perhaps worth considering for future studies requiring G.PS to access potentially suitable clients.

It was surprising that G.Ps' perceptions of being over-researched was the third mostcommonly-cited factor. One G.P. commented:

'Do you know how many requests we get to help on similar studies? About 1/wk (week). It's impossible'.

This is a pertinent issue for Ethical Committees and professional bodies to monitor.

\subsubsection{Prompted reasons for poor recruitment of clients}

The 12 factors that were listed as suggestions for poor recruitment were individually rated by G.Ps using the Likert scale (described in 8.3). These results were analysed by scoring the results from $1-4$ for each response and then adding the responses from the 24 G.Ps who completed the questionnaire. These totals were then ranked. Where a G.P. failed to use the numerical score, but placed a $\checkmark$ in the box, a constant of $2 \frac{1}{2}$ was scored as the median point of the scale. Missing data were ignored and thus scored 0 . The final results are shown in table 8.3 .

It is clear that pressure of work was again the main factor deemed responsible for the slow recruitment of clients into the study. The top five reasons given in table 8.3 mirrored the top reasons given in table 8.2 , although when unprompted, the time taken to enter a client was only ranked fifth, two places below table 8.3.

Reasons included in the 'other' category were either 'patient benefit' (total score $=6$ ) and one score of 1 which was not specified. 


\section{Table 8.3: Ranked reasons for poor recruitment of clients}

Results from listing 12 factors of possible reasons for the slow recruitment of clients in to the 'Back Home' study. G.Ps were asked to identify the extent to which they thought each factor contributed to this issue using a Likert scale where $1=$ 'not at all', $2=$ 'mildly', 3 = 'moderately' and 4 = 'very significantly'.

\begin{tabular}{|c|l|c|}
\hline Rank & Factor & Total score \\
\hline 1 & Pressure of work & 60 \\
\hline 2 & G.Ps forgot to include potentially suitable patients & 52 \\
\hline 3 & Process of entering a patient was too time-consuming & 39 \\
\hline 4 & G.Ps are over-researched & 31 \\
\hline 5 & Exclusion criteria prevented inclusion of many patients & $30^{1 / 2}$ \\
\hline 6 & G.Ps had to decide when to enter a patient for the study & 30 \\
\hline 7 & Other research projects also underway in your practice & 29 \\
\hline 8 & It was difficult to find the paperwork & 27 \\
\hline 9 & The research was hospital-led & $231 / 2$ \\
\hline 10 & Randomization occurred at the practice rather than patient level & 15 \\
\hline 11 & Research team was multidisciplinary & 14 \\
\hline 13 & Study design (i.e. a randomized controlled trial) & 7 \\
\hline
\end{tabular}

\subsubsection{Any other comments}

For this section, a total of eight comments were received, covering issues about the study design, for example two G.Ps in the control group commented there were "no clear benefits to patients taking part' and one G.P. found the paperwork was 'difficult to follow'.

Other comments were particularly positive about the leaflet and two G.Ps asked whether they could use it in their surgery for clients with back pain. One G.P. reflected on their own clinical practise and reported that they 'would not normally consider referring single episode', although this was ambiguous as it was unclear what the G.P. was considering referring the client to. 
Finally two comments were made which reflected positively on the research:

'I enjoyed participating'

'Thank you for your hard work.'

\subsection{The implications of these findings for future research}

This small study provided useful information, both for the research team and project sponsors, as to why recruitment was problematic from the G.Ps' perspective.

Pressure of work was clearly seen as the major factor, which raises issues for funding future projects that require G.Ps to access clients through their regular surgeries. This issue may seriously threaten the future of primary care research and perhaps the reason it has not had more impact to date is that at present, funding for primary care research comes from a number of sources. However the warning signs must surely be evident to funding agencies - increasing the duration of studies, expanding catchment areas, revising inclusion criteria etc. It is likely that if this trend continues, studies may soon actually fail for these very reasons.

\subsubsection{The composition of the research team}

Perhaps one determinant of "success" is the professional background of the research team (including the researcher). However, perhaps recruitment would have been less problematic had a doctor initially recruited G.Ps into the study rather than a chartered physiotherapist?

Whilst many health care professionals welcome interdisciplinary initiatives, it would seem likely that they can best identify with individuals from their own professional background. Therefore perhaps it is worth considering this in future when approaching potential participants.

\subsubsection{The use of incentives}

Another means of potentially increasing participation in studies is through the use of incentives. Some may view this as a practical means of potentially improving recruitment rates in a world of increasing accountability; others may view it as a form of bribery. To date little is published on the use of incentives in primary care. That which 
does exist, tends to focus on giving incentives in research as a means of improving compliance and retention of study participants ${ }^{(5)}$, with no mention of giving incentives to G.Ps who recruit clients.

It has been claimed that small incentives have generally proved helpful but larger incentives often are not helpful in increasing response rates ${ }^{(3)}$. However it is more likely, as Rudy says 'To what extent these incentives really work, and which incentives work best, is not known'(5). Some factors that influence participation are the amount and type of payment, the extent of any risk involved, and the neediness of the participant $t^{(5)}$.

Using incentives is a difficult issue which could be seen as recompensing G.Ps for undertaking additional work on behalf of a study, or even as a means of income generation. On the other hand, it may jeopardize the future of primary care research if G.Ps are only persuaded to participate by the offer of incentives. There is also a moral dimension, since health professionals may perceive themselves duty-bound to the advancement of knowledge through research. This issue is not unique to G.Ps - many other health professionals also participate in research.

As well as being ethically sensitive, using incentives (or not) is likely to evoke strong sentiments from G.Ps. Even in our pilot study (cited in chapter three) one G.P. commented:

'This is one amongst many similar requests for my time. I have much sympathy with those GPs who routinely file such unpaid work requests in the bin!'

Aware of this controversy, part-way through our study (when the slow recruitment was obvious, it was decided to give G.Ps a bottle of wine as a gesture of thanks when they had recruited six clients into the study. This was funded by external sources i.e. not the research grant. Due to its late timing in the study, this was relatively ineffective as an 'incentive', although it did serve as a physical expression of our gratitude which was welcomed by the recipients! 


\subsection{Summary of the chapter}

- Research studies which necessitate G.Ps accessing clients from their regular surgeries are likely to experience difficulties with recruitment, which has implications for funding agencies and the advancement of knowledge.

- Pressure of work was consistently the most-commonly-cited factor for poor recruitment of clients, by G.Ps in our research.

- Composition of research teams and the use of incentives may affect recruitment rates. 


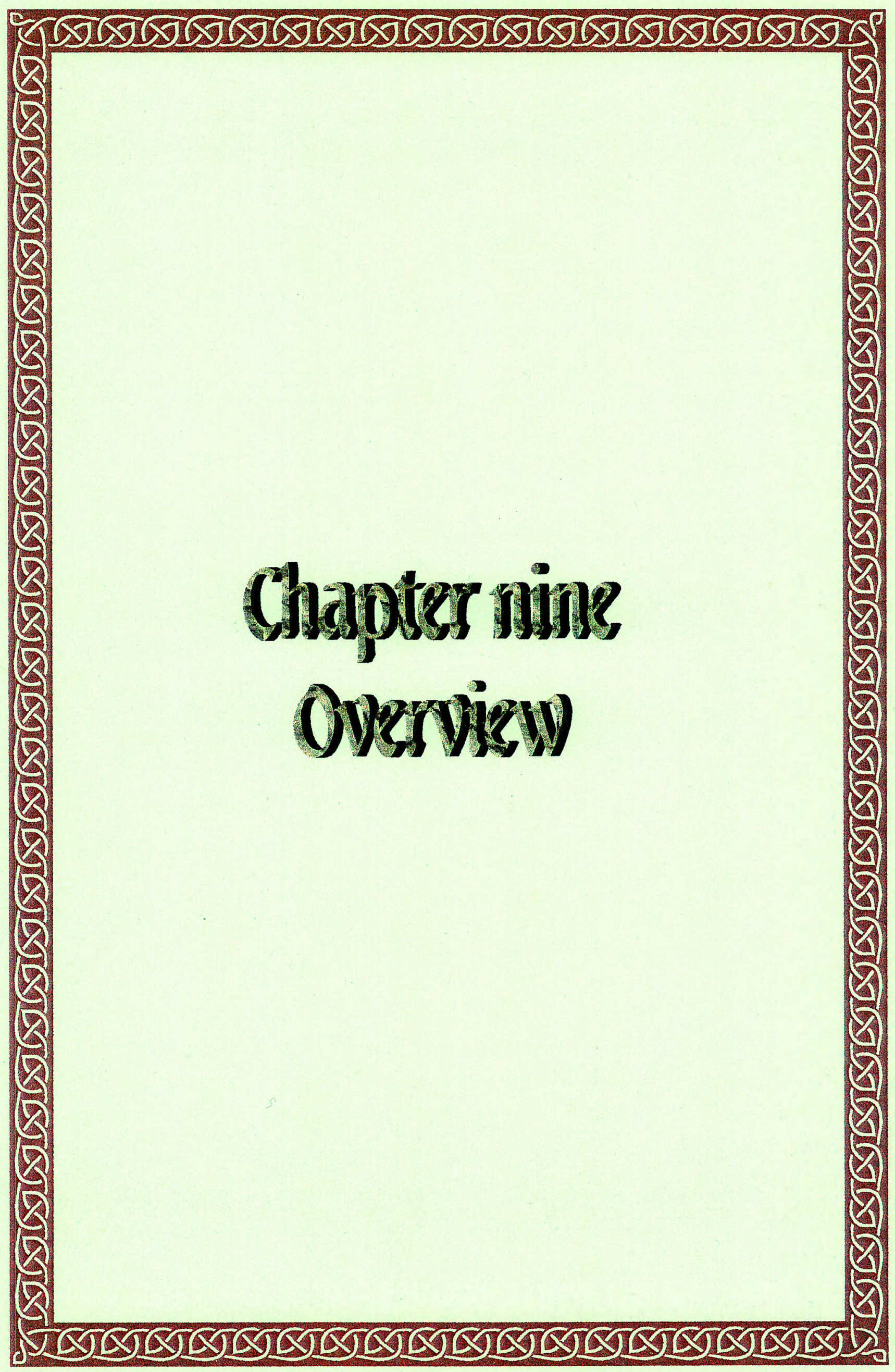




\section{Chapter nine: Overview}

Having considered the detail of the four studies in this thesis, the final chapter aims to draw together the issues in the preceding pages, and consider the research as a whole. It summarizes assumptions made, areas of tension that have occurred during this work and limitations of the studies completed, before outlining plans for future work.

The chapter closes with conclusions from the research undertaken and finally, from the researcher's perspective, a personal reflection on the process of actually completing this work.

\subsection{Assumptions}

The most important assumption made in this piece of work was that acute low back pain can be measured (and furthermore it can be measured by using self-report outcome measures). At the end of this thesis, these assumptions are still pertinent, although as discussed in chapter four, the self-report instruments currently available for measuring acute low back pain are generally crude and psychometrically deficient. There is much potential for development and refinement of these outcome measures, which should be a priority for all who are interested in studying back pain, otherwise how can any interventions be evaluated, if their impact cannot be monitored?

At the start of this research, the locus of control concept was assumed to be an important factor when studying clients with acute low back pain. It is evident, particularly from the work in chapter seven, that perceptions of control are important, although the locus of control construct does not provide all the answers. It is possible that this is just part of a larger psychosocial construct, which also includes illness perceptions, attitudes, beliefs, state / trait personality characteristics and perceptions about treatment. However, this is merely speculation, which cannot be concluded from this piece of work. In addition, it must be remembered from chapters four and six, that the existing instruments, which were developed to measure locus of control, are flawed.

Another assumption, directly relating to back pain, was the natural history of this symptom. From the reviews which were available at the start of the research, it was 
anticipated that in the majority of clients, their acute low back pain episode would rapidly resolve — the Quebec Task Force for Spinal Disorders reported that ' $74 \%$.... resolves within four weeks and $87 \%$ within three months ${ }^{\text {(1) }}$. Such reports influenced the timing of data collection in the 'Back Home' study, so that an initial assessment was made as soon as possible after the client visited their G.P. (within two working days), with the main assessment at two weeks and a further follow-up at three months.

However, the more recent literature such as the Clinical Standards Advisory Group report ${ }^{(2)}$ and a paper by Croft et al. ${ }^{(3)}$, have challenged these 'over-optimistic' earlier estimates $^{(2)}$. They conclude that clients have stopped consulting with symptoms within three months, although 'most will still be experiencing low back pain and related disability one year after consultation'(3). Had this evidence been available when the 'Back Home' study was designed, it is likely that the main assessment would have been delayed, probably until six weeks (in line with recommendations in the C.S.A.G. report). (The data collection was extended to include further follow-up assessments at six and twelve months, when the work focusing on control issues, was designed, which was fortunate as the data were then available for these clients over a one-year period.)

Having considered assumptions which have directly related to back pain, it is important to consider those that relate to the G.Ps and clients who participated in the study.

Firstly, in any research project that requires people to take part, there is an inherent assumption that clients do want to be involved. Unfortunately, we do not have data for any clients who were approached by their G.P. and declined to participate, as it was essential to minimize the involvement of the G.P. However, in table 8.2, two G.Ps, out of the 24 who responded to the follow-up questionnaire on recruitment, identified this issue as a reason for slow recruitment of clients into the study.

Ideally it would be interesting to review the medical notes of the clients who declined to participate, to measure whether they reported any further recurrence of their symptoms. However, the pragmatic issue of burdening G.Ps outweighed the benefits of such information, and therefore this information was not collected.

It was also assumed that clients would want to stop their involvement with the study after the final assessment. Surprisingly, this was not always the case and this issue is discussed further in section 9.3.6. 
In addition to assumptions about participating in the study, a minority of clients also appeared to have assumptions that any information they gave might be used to review the financial benefits they were currently receiving, or the status of any potential litigation. (However, if clients did pursue any legal action, they were subsequently excluded from the study.) Such clients, who were suspicious of the motivation for the study, were initially somewhat reticent in their answers. It was interesting to note that when they were assured that the research was independent from such agencies, and that any information they chose to declare would be treated anonymously and confidentially, there was a notable change in their attitude. An example of this change in attitude was seen when a client, who had reported the immediate onset of back pain after lifting a sack of nuts which he 'threw down', remarked:

'I'm thinkin' about claimin' for this injury 'cos it happened at work, only don't tell me boss that I done it 'cos I lifted badly'.

Further assumptions were made in this research regarding the participating G.Ps. The assumption was (perhaps naïvely) made that G.Ps who agreed to participate in the study, would then proceed to recruit clients. However, from the work in chapter eight, this clearly did not occur in many cases, hence the follow-up study.

\subsection{Key issues arising from the research}

Although the emerging key issues have been addressed in the preceding chapters, the main areas of development of theory in this work surround control issues and outcome measures, which are reiterated here.

\subsubsection{Control issues}

Clients perceive their ability to control their acute low back pain as considerably different to their ability to control their general health; although these differences become less with time. As a result of this, it is important to use outcome measures of control which have been specifically validated with this client group.

When considering the locus of control construct, it is important to remember that the three subscales (internal, external [powerful others] and external [chance]) are not 
mutually exclusive - an increase on the internal subscale does not result in an automatic decrease on the external subscales.

Overall, in our population of clients with acute low back pain, using the conditionspecific M.H.L.C. (form C), generally clients scored higher on the internal subscale than the external scales, therefore perceiving their own actions to be a greater influence on their back pain than either 'powerful other' or 'chance' factors.

Gender differences were also apparent in these results, with males scoring more highly on all the external subscales of the M.H.L.C. (form C) than females. Therefore, male clients perceived a greater influence of 'powerful other' and 'chance' factors in their low back pain than female clients. These results were not affected by whether (or not) clients were able to establish a cause for their symptoms.

The popularity of the locus of control construct is waning and criticism of this notion has become widespread, so that the construct is perceived as a portion of the broader issue of 'perceived control over health'(4). It is unclear whether this loss of favour stems from limitations of the construct itself, the way it is measured or its application in clinical practise. It is likely that all three aspects have contributed to its current demise.

What is clear is the complexity of measuring control issues and the need for further work in this area.

\subsubsection{Outcome measures}

There are many factors to consider when selecting appropriate outcome measures, both for research and clinical practise. These factors can be divided in the '3Ps': purpose (i.e. ability to measure dimensions of health); practical aspects; and psychometric properties (including reliability, validity and sensitivity to change).

All of these factors need to be weighed up in the context of the study design or clinical setting, for the particular population under review. It is essential that outcome measures continue to be developed and revised, since physiotherapists cannot practise unless we take measurements ${ }^{(5)}$. Therefore these measurements need to be accurate and meaningful. 


\subsection{Tensions}

There were several sources of tension throughout this research, some pertaining to the study itself, such as the role of the researcher and safety issues, whilst others were more to do with the research process and included cross-faculty research and issues of parttime study.

\subsubsection{The role of the researcher}

A particularly challenging aspect of the work was balancing the tension of being a clinician and researcher. The sheer frustration of visiting clients at home (whose lives were adversely affected by their current episode of pain), knowing that clinically there was a repertoire of treatments and advice available which may be of benefit, and yet this desire to help had to be completely suppressed, as the role of being a researcher necessitated data collection without active intervention, and thus any attempts to offer advice or intervention would contaminate the results.

There were times when this tension was very uncomfortable, challenging both ethical and professional standpoints. On reflection, even by the end of the study, I had still not readily accommodated to this role of purely a researcher, and was still uncomfortable with this conflict.

\subsubsection{Teamwork}

It has been invaluable experience working as part of a team, being able to debate ideas and learn from each other. However, as in any group situation, not all opinions were unanimous, and the process of negotiation was, at times, complex. An example of this was the range of possible of incentives which were suggested to improve G.P. recruitment of clients into the study. Ideas ranged from: no incentive at all; a bottle of wine; a draw for a case of wine; to even sending a plastic duck to all G.Ps who had failed to recruit any clients at all! Such diverse views were reconciled by negotiation and sometimes lively debate, and it was important that the team worked well together, especially through the frustrations of the slow recruitment of clients into the study.

The multi-professional nature of the team also played a vital role. With half the team currently practising clinically in three different professions (physiotherapy, rheumatology 
and general practice), this ensured the research retained a pragmatic focus. Being a multi-professional team however, was not always advantageous. Initially there was some debate regarding the study design, when one profession was perceived by another to dominate. Successfully resolving such issues was all part of the team-building process.

On reflection, perhaps obtaining a greater balance between primary and secondary care team members might have been beneficial, thus giving G.Ps a higher profile in this research, which was after all, taking place in primary care. One disadvantage of this could be having many more than six members in the research team, since it might be difficult to represent everyone's views (having already had some experience of this in a team of six). It could be challenged whether three physiotherapists were necessary, or whether one (or more) could be replaced with primary health care professionals, but in fact, one of these three operated primarily in a managerial role, and thus the degree of overlap of roles was reduced. Therefore, if additional primary health care representatives joined the research team, in addition, there would be the practical issues of organizing meetings with larger numbers of people.

Perhaps as a means of giving G.Ps a higher profile in the research, as discussed in 8.5.1, a G.P. could have initially recruited the doctors into the study. Indeed, this has proved very successful in a subsequent study, involving some of the 'Back Home' team, where over 300 clients have been recruited from two geographical areas where the G.P. engaged other doctors in the initial phase of the study, as opposed to a chartered physiotherapist.

\subsubsection{Safety issues}

Whilst actually collecting data, there were safety issues around completing field-work in clients' homes. This was not an issue to which the team had given much attention, although on reflection, this was an oversight. Clearly there was a certain vulnerability about going into clients' homes for repeated visits, uncertain of what to expect. Following discussions with health professionals who undertake domicillary work, obvious precautions were taken, such as taking a (hospital) mobile telephone, personal alarm and leaving details of where visits were taking place. However, the client group in the study were not directly comparable to the population to whom domicillary visits are usually made, being younger, physically stronger and often asymptomatic at the time of many of the visits. 
An unnerving experience on one of the earliest home visits reinforced this vulnerability and constant need for vigilance. Follow-up visits to this particular client in future always had the additional precaution of an escort for the researcher, and a pre-arranged routine for the researcher (and escort) to be contacted by mobile phone, if they had not reported in to the hospital by an agreed time.

Safety issues are rarely discussed in texts on research methods, and they must be addressed as an essential component of any research project. This is pertinent where researchers will be working alone, visiting people in the community, particularly in social science research, where many researchers are likely to be young and female. However any researchers working alone are potentially vulnerable. This issue has been raised formally, both at the University and the Southampton and South West Hants Joint Research Ethics Committee, who have a responsibility, not only for protecting the interests of the research participants, but also of those who undertake it.

\subsubsection{Pragmatism versus idealism}

One of the recurring themes throughout this thesis was the tension between ideal or 'best practice', and actually doing pragmatic research. Nowhere was this more evident than in the initial stages when it was necessary to start the G.P. survey and 'Back Home' study as soon as possible to ensure the work was completed within the period of funding.

Three months into this work, the PhD commenced, but the two time frames for these two pieces of work were not concurrent. On the one hand, the issues for the $\mathrm{PhD}$ (especially around outcome measures and control issues) would benefit from time to read extensively around the subjects and reflect, whereas the 'Back Home' study needed to be underway as soon as possible to ensure the study could be completed within the funding constraints.

In addition to issues of timing, further tensions arose around selecting a sample for the pilot G.P. questionnaire. In section 3.3.2, the issue was raised of using a different sample of G.Ps for the pilot questionnaire (i.e. G.Ps practising in Salisbury as opposed to Southampton, where the main study was to take place). In an 'ideal' world, the sample chosen for the pilot study should be a subgroup from the main population being studied, but in reality, it was considered more important to minimize possible sources of 
contamination and maximize the number of G.Ps available for the final survey, than adopting what might be considered 'best practise'.

In section 5.10.2, the conflict of baseline measurements and interventions was discussed. Ideally, it would be preferable to collect baseline data from clients prior to any intervention, such as the 'Back Home' leaflet. However, clinical practise suggests that if a G.P. chooses to give a client an advice leaflet, they are likely to use it at the initial consultation, where the G.P. may empower the client to read the leaflet and adopt its content. This would inevitably be before any baseline measurements could be taken. Thus a compromise was reached, where the method was designed to reflect clinical practise, in preference to collecting truly baseline data.

Further tensions between being pragmatic versus idealistic were seen in section 8.2 , where it was recognized that the G.P. questionnaire (establishing the current approach to the management of clients with acute low back pain) should be repeated at the end of the study to monitor any changes in G.Ps' approaches to back pain. Regrettably, the need to investigate the poor recruitment of clients into the study took priority over this issue.

Once again, compromise was necessary when working with G.Ps, regarding the followup questionnaire itself (see section 8.3). Here, it was important to collect information from G.Ps about why client recruitment into the study was so poor, and also to ascertain whether G.Ps were interested in receiving any of the reports or papers from the completed study. In an 'ideal' world, these requests for information should be issued separately, thus preserving anonymity of the participants in the follow-up questionnaire. However, the brevity and simplicity of collecting this data took priority over preserving anonymity, once again, weighing pragmatism against idealism.

\subsubsection{Quantity of data collection}

Having just considered pragmatic research, a very important tension involved the quantity of data being collected. This thesis has shown that acute low back pain is a multidimensional symptom, which makes it complex and challenging to study. Initially it was difficult to establish which dimensions should be measured and throughout this debate there was always a tension of how much data the team would like to collect versus how many outcome measures clients would be able to complete, especially in the early 
stages, where they may be too uncomfortable to physically sit down, and complete the questionnaires.

From the literature discussing the natural history of low back pain (see 2.7), the research team had anticipated that many clients would be considerably improved by two weeks (assessment two), which was thus the main assessment for collecting data. However, due to the inclusion criteria (in particular the prerequisite that clients must be taking at least three days off work or equivalent), our sample were often still experiencing moderate or severe discomfort at this time, which made data collection a lengthy process in some cases.

As the researcher 'at the coal-face', it was sometimes difficult to balance this tension. It was imperative to remain sensitive to clients' symptoms, but this potentially conflicted with trying to balance the desire for collecting data, both for the 'Back Home' study and $\mathrm{PhD}$ research. On a couple of occasions the researcher chose to terminate the visit without completing all the outcome measures, due to concerns about clients' symptoms and the time they had already invested in giving information.

\subsubsection{Negotiating an exit}

It was not only during the early home visits that tension arose. On a few occasions, negotiating an exit at the end of the study became difficult, particularly when clients lived alone and had shared a small part of their life with the researcher for twelve months. Although this had been recognized as a potential issue and the researcher had made a conscious effort not to raise clients' expectations, sometimes requests for further visits were made, and other gestures included a gift, and even an invite to a wedding! On reflection, at times, the inevitability of human interaction made negotiating an exit more difficult than had been anticipated, and some clients seemed disappointed that the study was finite.

\subsubsection{Cross-faculty research}

As well as tensions arising from the study design, there were also sources of tension arising from the process of actually doing the research.

One such tension was the need to cross professional and faculty boundaries, which, despite the advantages cited in chapter one, also produced areas of concern. For 
example, addressing control issues from a physiotherapy perspective was inevitably more familiar that adopting a social science perspective. This concern lessened with increased familiarity with social science literature and contact with health professionals from these disciplines - even deciding whether to call people with acute low back pain patients or clients was an issue.

\subsubsection{Issues of part-time study}

For eighteen months, the work in this thesis was undertaken alongside the 'Back Home' funded work, which started as a full-time venture. After this time, the work became 'part-time' to accommodate the slow recruitment of clients into the study, together with increasing clinical and teaching commitments.

The major advantage of continuing the research on a part-time basis was that it enabled the initial data collection to continue until December 1997. However, the tension arose of balancing research, clinical, teaching, scholarly and administrative commitments, which actually became the greatest challenge of the thesis. Nevertheless, such a pattern did enable some time for reflection and development of ideas, and having experienced both practises, there are clear advantages and disadvantages to both full and part-time study, and as circumstances changed, it was important to revise working practises where possible.

\subsection{Limitations}

Having acknowledged assumptions and sources of tension in this research, it is important to review the limitations of this work as a whole. (N.B. Issues that have been considered in some detail earlier in the thesis will only be briefly reviewed in this section.)

\subsubsection{Generalizability}

It must be remembered that whatever the findings from this work, the results represent views from one client group in Southampton and the New Forest.

Although the characteristics of this population were considered in chapter five, these cannot be assumed representative of the U.K. as a whole, since factors such as regional variations in G.P. consultations for back pain have been reported ${ }^{(6)}$, which were discussed 
further in section 3.1. Without further evidence from other cluster populations within the U.K., it would be incorrect to claim the results in this research were generalizable to the U.K. population.

\subsubsection{Level of randomization}

Another limitation of the study design that affected client recruitment into the study was the level of randomization (i.e. at the level of the G.P. practice) rather than at the individual G.P. or client level. This is discussed in detail in section 5.10.2.

\subsubsection{Sample size}

It was also suggested in section 5.10.2 that the relatively small numbers of clients in the control and experimental groups (28 and 35 respectively) was a limitation in this research. Small sample sizes reduce the confidence that can be placed in the results, thus suggesting that findings should be interpreted with caution. Possible ways of increasing sample size were discussed in chapter eight.

\subsubsection{Inability to follow-up non-responders}

Earlier, in section 9.1, the inability to follow-up clients who declined to participate in the 'Back Home' study was acknowledged, and although the reasons for this are obvious, omitting these data were nevertheless, a limitation of the study.

\subsection{Implications of findings}

Despite the limitations of this work, it is important to consider the implications of the research findings. After all, why are control issues important in clients with acute low back pain?

It has been reported that educational interventions such as Backschools can change clients' knowledge about back protection behaviours ${ }^{(7)}$, but the 'Back Home' study went further to show not only improved levels of knowledge when clients received an information leaflet, but also positive changes in their behaviour. Although this did not directly manifest as measurable improvements in clients' levels of function and hasten their return to work in this study, this warrants further work, since if information leaflets could influence knowledge, behaviour and function, this might result in health gain for 
the individual, and financial gains for the health services and society in general. It should be recognized that such gains are likely to be modest.

In addition, perhaps health professionals may feel reassured that by using information leaflets, they are able to impart more information to clients than would otherwise be possible during routine consultations, and this information can be re-read later. This may be a small factor which helps to alleviate some of the stress perceived by G.Ps, although it is imperative that information leaflets are not used as a substitute for good communication between the doctor and client ${ }^{(8)}$, remembering Hadler's words 'Does not everyone in pain deserve the empathy and human contact that can never occur in any "educational booklet?"”(9)

However, leaflets are only likely to be of use if a) the G.P. has read the content and can empower any salient points that are relevant to the client, b) the client reads and understands the content, c) the key messages are accurate, up-to-date and appropriately presented and d) the client wishes to take note of the content, and can relate it to their own life.

\subsection{Future work}

Following completion of this work, the first priority will be re-surveying the G.Ps to measure their current management of clients with acute low back pain. This will be a five-year follow-up for the work already published ${ }^{(10)}$, and will provide useful information on the impact of clinical guidelines such as those produced by the Clinical Standards Advisory Group ${ }^{(2)}$ and the Royal College of General Practitioners ${ }^{(1)}$, as the work reported in chapter three was prior to publication of these guidelines.

In addition, further work is anticipated to start in 2000, involving clients' beliefs and perceptions of control about their back pain, in contrast with others that are experiencing other musculoskeletal symptoms.

Ultimately a goal of this work would be to go back to the Backschool (which is where this work started) and revise both the content and presentation in the light of the research findings. 
Preliminary work has already begun, in the form of supervision of an undergraduate dissertation, which investigated clients' expectations before they attended a Backschool and whether these expectations were subsequently met $^{(12)}$. Following on from this work, supervision of a second project has recently been completed which has explored undergraduate students' attitudes towards Backschools ${ }^{(13)}$. The next link in this chain would be to ascertain attitudes of qualified clinicians towards back education, so that this evidence could be used alongside the work in this thesis to revise the content and presentation of the Backschool. Additional resources could be used such as the knowledge gained from the supervision of a recent Masters dissertation, which investigated how physiotherapy can meet the needs of people with chronic low back pain in an outpatient department ${ }^{(14)}$. This work will be useful, since the results of this study suggested that clients who received a cognitive-behavioural approach to treatment were likely to show greater functional improvements than clients who received mobilisation, exercises and advice (despite their pain levels remaining relatively high).

What is particularly advantageous is that both the study involving clients' expectations and the evaluation of the cognitive-behavioural approach took place in the same physiotherapy department, which minimizes differences in working practises between departments.

Having considered Backschools from clients', clinicians' and students' perspectives, further qualitative work would then need to be done, perhaps in the form of focus groups, to ensure that the Backschool material fully addressed the clients' agendas, which is paramount (as was seen in the development of the 'Back Home' leaflet in chapter five).

Apart from the future research work which is planned, priorities for dissemination include the findings from the control study and the systematic review of outcome measures.

\subsection{Conclusions}

Perhaps the most striking finding from the work in this thesis is the complexity of measuring psychosocial aspects of clients with acute low back pain. This challenge is best approached from a multi-professional perspective, particularly one that spans several fields, including both social and health sciences. 
Before the psychosocial aspects can be addressed, it is paramount that any research which studies low back pain includes a precise definition of the anatomical boundaries of the area under review, the sensations that are included, together with any exclusion criteria. Only then can study findings be meaningful. A standardized definition would greatly enhance comparability between studies, evaluate interventions and enable collection of accurate epidemiological data. The anatomical area proposed is that bounded by the $12^{\text {th }}$ thoracic vertebra and $12^{\text {th }}$ ribs superiorly, gluteal folds inferiorly and contours of the trunk laterally. The length of time that the client has experienced these symptoms should also be specified.

At the heart of clinical practise is the concept of a thorough assessment, which incorporates both client interview and examination. It is absolutely essential the clinician ensures that clients' symptoms are not likely to be caused by serious spinal pathology. Guidelines are being developed to assist clinicians in identifying these 'danger signs' or 'red flags', but it is important that these guidelines do not lure clinicians into a false sense of security, as they are not definitive. Vigilance is essential, as the consequences of failing to detect such pathology are potentially disastrous.

It is important that psychosocial factors are considered too, ensuring that a truly biopsychosocial approach is adopted. In the most recent clinical guidelines, psychosocial or 'yellow flags' have been addressed ${ }^{(15)}$, and it is likely that these will become increasingly important in the future as their full impact is recognized.

In both the research and clinical setting, measuring low back pain is an on-going challenge. Although much work has already been done on the development of outcome measures, there is still much to do. Being able to measure back pain is absolutely fundamental - it is the cornerstone to researching back pain, evaluating management strategies and clinical interventions. Only then can effectiveness be determined.

So far, these conclusions have considered issues primarily focused on the health professional. However, it is just as important to consider clients' perceived control over their health, which at present cannot easily be determined. Such perceptions are an evolving notion, and it has only been recently that their importance has been recognized. By understanding the clients' perspective, the clinician can start to unpack the complex, 
psychosocial issues, which are inherent in any client with low back pain. There is no better context in which to study these issues than within the social sciences.

\subsection{Summary of the chapter}

- An important part of any piece of research is to identify any assumptions made and limitations of the work undertaken, as these can influence the interpretation of the findings, and subsequent conclusions.

- Careful consideration was necessary throughout this work, to balance the desire to use 'ideal' research practises, whilst completing this work in a real-world, primary care situation, where at times, the need to be pragmatic was paramount.

- Researchers, particularly those who work alone, visiting people in the community, need to be aware of safety issues associated with this work, and take appropriate precautions to ensure their personal safety. This responsibility for personal safety also extends to other members of the research team, supporting agency and Ethics Committee, who endorse this work. 


\subsection{Personal reflections ..... alone in a crowd}

This research has been a new experience, which at times has been highly challenging. It has been an opportunity for me to learn, not only about acute back pain and psychosocial issues, but also to explore and challenge my own thoughts and assumptions.

The study was a learning experience both conceptually and practically. It was extremely beneficial (as a clinician) to observe people in their home environment, witnessing their behaviour, modifications to their lifestyle and sometimes, interactions with other family members or friends. This experience still impacts upon my current clinical practise, paying greater emphasis to clients' social situations and support networks. On reflection, I am aware that my own views have shifted, now valuing more highly the role of more qualitative data (such as field notes) and the importance of clients' beliefs. Indeed, one of the most interesting aspects of data collection was clients' informal comments, some of which have been included in this thesis.

Looking back at the research process, it has seemed analogous to a journey. There have been many twists and turns, a few wrong turnings, as well as the occasional detour. Now, nearing the end of this stage, the journey has been different to that which I expected to take at the start. It was a less direct route than anticipated, with more to see along the way. At times the road ahead was unclear, especially when selecting outcome measures, whilst at other times, the road did not appear to exist, in particular on two occasions: firstly when combining issues of control and acute low back pain; and secondly when approaching issues from a physiotherapy and social work perspective. New roads needed to be built to enable the journey to continue.

Although this journey has been made alone, it has been alone in a crowd, as there have been many useful signposts and sources of guidance along the way, as well as fellow travellers that have shared parts of the experience. I have learned much from this venture, and am deeply grateful to my navigators and recovery service who have helped me get this far! As the road stretches out ahead, the journey does not end here - this is only the beginning. There is still much more to learn, but regarding these first tentative footsteps, as Shakespeare ${ }^{(16)}$ said:

what's done cannot be undone: to bed, to bed, to bed.' 


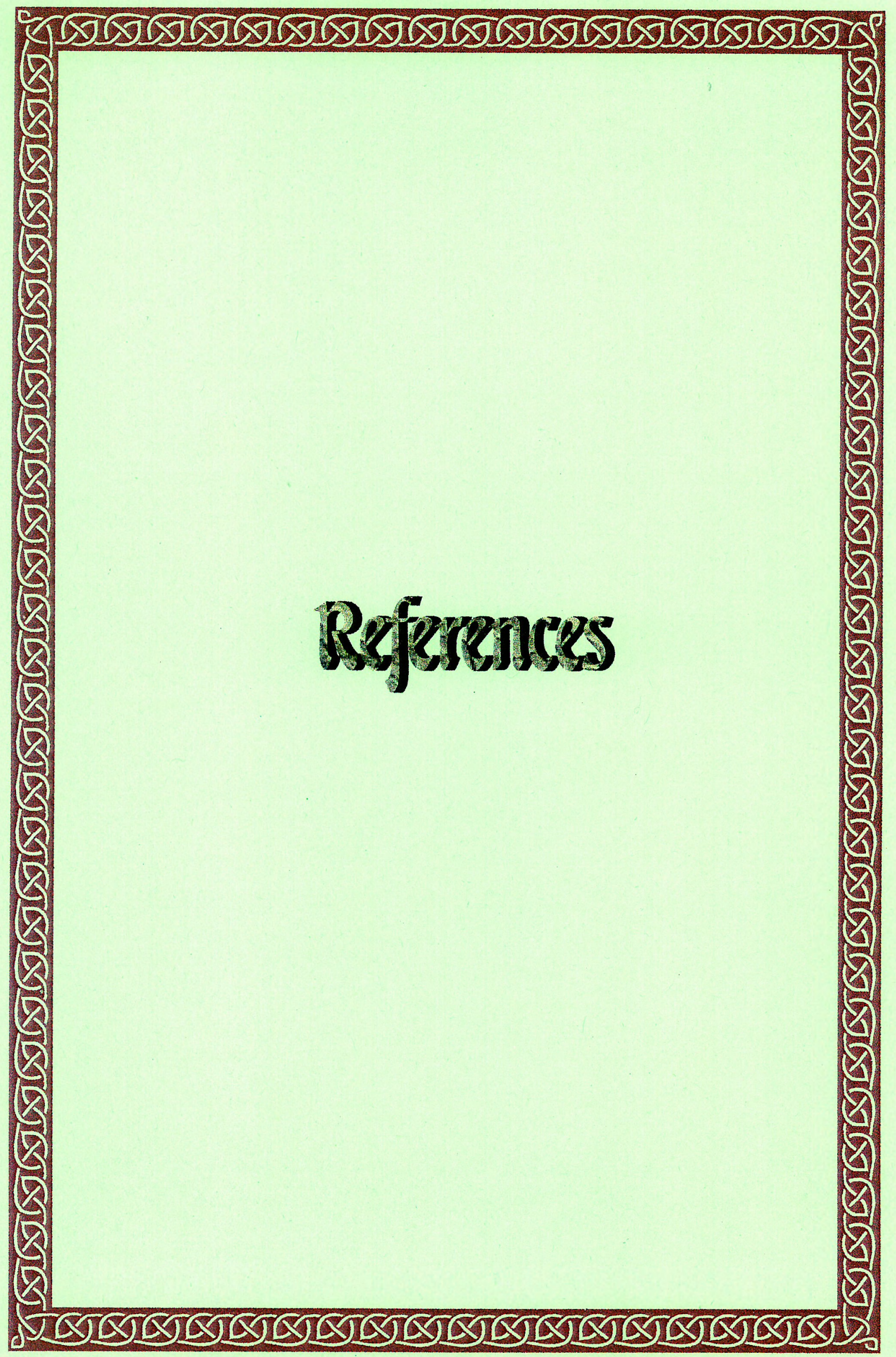




\section{References}

\section{Chapter 1: Introduction}

1. Klaber Moffett J.A. 1994 The role of psychological variables in the assessment and physiotherapeutic management of musculoskeletal disorders. $\mathrm{PhD}$ Thesis, University of London, pp18-9.

2. Kvale S. 1992 Postmodern psychology: A contradiction in terms? In: Psychology and postmodernism Steinar Kvale (ed.). Sage, London pp31-9.

3. Biggs S. User voice, interprofessionalism and postmodernity. Journal of Interprofessional Care 1997;11(2):195-203.

4. Howe D. Modernity, postmodernity and social work. British Journal of Social Work 1994;24:513-32.

5. Waddell G. 1998 The back pain revolution. Churchill Livingstone, Edinburgh pp228, 276-7.

6. Delitto A. Are measures of function and disability important in low back care? Physical Therapy 1994;74:452-62.

7. Waddell G. A new clinical model for the treatment of low-back pain. Spine 1987;12(7):632-44.

8. Gordon S. 1991 Sociality and social science. In: The history and philosophy of social science. Routledge, London pp1-15.

9. Oxford Compact English Dictionary 1996 Oxford University Press, Oxford pp782.

10.Open University School of Health and Social Welfare. $1998 \mathrm{New}$ directions for good practice in K302: Enhancing critical practice in health and social care pp2-22.

11.Bauman Z. 1995 Life in fragments. Essays in postmodern morality. Blackwell Publishers Ltd., Oxford pp80-2.

12.Gale T. 1993 Reflections of an old lag. Discussion paper for the Faculty Research Training Scheme. Department of Psychology, University of Southampton pp1-9.

13.Hicks C.M. 1995 Research for physiotherapists. Project design and analysis. Churchill Livingstone, Edinburgh 2nd edition pp6.

14. Wallston K.A. Personal communication (see appendix 6.1)

15.Spitzer W.O., LeBlanc F.E., Dupuis M. et al. Scientific approach to the assessment and management of activity-related spinal disorders. Spine 1987;12(7S):S1-S59.

16.The Health of the Nation, a strategy for health in England. 1992 HMSO, London pp 16,42 .

17.Agency for Health Care Policy and Research. 1994 Management guidelines for acute low back pain. U.S. Department of Health and Human Services, Rockville MD.

18.Clinical Standards Advisory Group. 1994 Back pain. Report of a CSAG committee on back pain. HMSO., London. 
19.Waddell G, Feder G, McIntosh A, Lewis M, Hutchinson A 1996. Low back pain evidence review. London: Royal College of General Practitioners pp1-34.

20.Kendall N.A.S., Linton S.J., Main C.J. 1997 Guide to assessing psychosocial yellow flags in acute low back pain: Risk factors for long-term disability and work loss. Accident Rehabilitation and Compensation Insurance Corporation of New Zealand and the National Health Committee, Wellington, New Zealand pp1-22.

21.Evans G., Richards S. 1996 Low back pain: an evaluation of therapeutic interventions. Health Care Evaluation Unit, University of Bristol pp8-15.

22.Wallston K.A., Wallston B.S., DeVellis R. Development of the multidimensional health locus of control (M.H.L.C.) scales. Health Education Monographs 1978;6(2):160-70.

\section{Chapter 2: Review of the literature}

1. Walsh K. 1992 An epidemiological study of low back pain. Doctoral Thesis, University of Southampton, Faculty of Medicine.

2. Kelsey J.L., White A.A. Epidemiology and impact of low-back pain. Spine $1980 ; 5(2): 133-42$.

3. Deyo R.A., Tsui-Wu Y.J. Descriptive epidemiology of low-back pain and its related medical care in the United States. Spine 1987;12(3):264-8.

4. Jayson M.I.V. 1992 Back pain. The facts. Oxford Medical Publications, Oxford. 3rd edition pp18-28.

5. Skovron M.L. Epidemiology of low back pain. Baillière's Clinical Rheumatology 1992;6(3):559-73.

6. Kuorinka I., Jonsson B., Kilborn A. et al. Standardised nordic questionnaires for the analysis of musculoskeletal symptoms. Applied Ergonomics 1987;18:233-7.

7. Anderson J.A.D. Epidemiological aspects of back pain. Journal of the Society of Occupational Medicine 1986;36:90-4.

8. Biering-Sørensen F. Low back trouble in a general population of 30-, 40-, 50-, and 60-year-old men and women. Danish Medical Bulletin 1982;29(6):289-99.

9. Bergquist-Ullman M., Larsson U. Acute low back pain in industry. Acta Orthopaedica Scandinavica 1977;Supp 170:1-117.

10.Magora A. Investigation of the relation between low back pain and occupation. Industrial Medicine 1970;39(11):31-7.

11.Valkenburg H.A., Haanen H.C.M. 1982 The epidemiology of low back pain. In: Proceedings of the American Academy of Orthopaedic Surgeons Symposium on low back pain. CV Mosby Co., St. Louis pp9-22.

12.Spitzer W.O., LeBlanc F.E., Dupuis M. et al. Scientific approach to the assessment and management of activity-related spinal disorders. Spine 1987;12(7S):S1-S59.

13.Klaber Moffett J., Richardson G., Sheldon T.A., Maynard A. 1995 Back pain. Its management and cost to society. Discussion paper 129, Centre for Health Economics, University of York pp5-67. 
14.Biering-Sørensen F. A prospective study of low back pain in a general population. I: Occurrence, recurrence and aetiology. Scandinavian Journal of Rehabilitation Medicine 1983;15:71-9.

15.Nagi S.Z., Riley L.E., Newby L.G. A social epidemiology of back pain in a general population. Journal of Chronic Diseases 1973;26:769-79.

16.Horal J. The clinical appearance of low back disorders in the city of Gothenburg, Sweden. Acta Orthopaedica Scandinavica 1969;Supp 118:1-109.

17.Nohejl J., Dostãl C., Fãberovã R., Malecek J., Roth Z., Tmavsky K. Preliminary results of an epidemiological study of back pain in a Prague population. Czechoslovak Medicine 1987;10:117-24.

18.Hult L. The Munkfors Investigation. Acta Orthopaedica Scandinavica 1954;Supp 16:1-76.

19.Cassidy J.D., Wedge J.H. 1988 The epidemiology and natural history of low back pain and spinal degeneration. In: Kirkaldy-Willis W.H. (ed.) Managing low back pain Churchill Livingstone, New York. $2^{\text {nd }}$ edition pp3-14.

20.Burton K.A., Tillotson K.M., Main C.J., Hollis S. Psychological predictors of outcome in acute and subchronic low back trouble. Spine 1995;20(6):722-8.

21.Waddell G., Feder G., McIntosh A., Lewis M., Hutchinson A. 1996. Low back pain evidence review. London: Royal College of General Practitioners. pp1-34.

22.A.C.C. and the National Health Committee 1997. New Zealand acute low back pain guide. Wellington, NZ. pp1-13.

23.Carey T.S., Evans A.T., Hadler N.M., Lieberman G., Kalsbeek W.D., Jackman A.M., Fryer J.G., McNutt R.A. Acute severe low back pain. A population-based study of prevalence and care-seeking. Spine 1996;21(3):339-44.

24.Kendall N.A.S., Linton S.J., Main C.J. 1997 Guide to assessing psychosocial yellow flags in acute low back pain: Risk factors for long-term disability and work loss. Accident Rehabilitation and Compensation Insurance Corporation of New Zealand and the National Health Committee, Wellington, New Zealand pp1-22.

25.Anderson J.A.D. Problems of classification of low back pain. Rheumatology and Rehabilitation 1977;16:34-6.

26.Anon. Progress in back pain? The Lancet 1981;1:977-9.

27.Roland M.O. The natural history of back pain. The Practitioner 1983;227:1119-22.

28. Waddell G. A new clinical model for the treatment of low-back pain. Spine 1987;12(7):632-44.

29.Fry J. The 'acute back'. 1974 In: Fry J. (ed.) Common diseases, their nature, incidence and care. Medical and Technical Publishing Co. Ltd., Lancaster pp157-63.

30.Frymoyer J.W., Pope M.H., Clements J.H., Wilder D.G., Macpherson B., Ashikaga T. Risk factors in low back pain. Journal of Bone and Joint Surgery 1983;65A(2):213-8.

31.Barker M.E. Pain in the back and leg: a general practice survey. Rheumatology and Rehabilitation 1977;16:37-45. 
32.Bernard T., Kirkaldy Willis W.H. Recognizing specific characteristics of nonspecific low back pain. Clinical Orthopaedics and Related Research. 1987;217:26680.

33.Currey H.L.F., Greenwood R.M., Lloyd G.G., Murray R.S. A prospective study of low back pain. Rheumatology and Rehabilitation 1979;18:94-104.

34.Riddle D.L. Classification and low back pain: A review of the literature and critical analysis of selected systems. Physical Therapy 1998;78(7):708-37.

35.Clinical Standards Advisory Group. 1994 Back pain. Report of a CSAG committee on back pain. HMSO., London.

36.Agency for Health Care Policy and Research. 1994 Management guidelines for acute low back pain. U.S. Department of Health and Human Services, Rockville MD.

37.Langworthy J.M., Breen A.C. Rationalizing back pain: The development of a classification system through cluster analysis. Journal of Manipulative and Physiological Therapeutics 1997;20(5):303-10.

38.Nachemson A.L. 1995 Low back pain in the industrial world. An overview with recommendations for solutions. In: Proceedings of the 'Low back pain' conference of the Arthritis and Rheumatism Council, Aberdeen pp1-5.

39.Reisbord L.S., Greenland S. Factors associated with self-report back-pain prevalence: A population-based study. Journal of Chronic Diseases 1985;38(8):691-702.

40.Saraste H., Hultman G. Life conditions of persons with and without low-back pain. Scand. Rehab. Med. 1987;19:109-13.

41.Mason V. 1994 The prevalence of back pain in Great Britain. A report on O.P.C.S. Omnibus Survey data produced on behalf of the Department of Health. HMSO, London.

42.Bergenudd H., Nilsson B. Back pain in middle age: Occupational workload and psychologic factors: An epidemiological survey. Spine 1988;13(1):56-80.

43.Armenian H.K., Halabi S.S., Khlat M. Epidemiology of primary health problems in Beirut. Journal of Epidemiology and Community Health 1989;43:315-8.

44.Dunnell K., Cartwright A. 1972 Medicine takers, prescribers and hoarders. Routledge And Kegan Paul, London pp8-22.

45.Heliövaara M., Sievers K., Impivaara O., Maatela J., Knekt P., Mäkelä M., Aromaa A. Descriptive epidemiology and public health aspects of low back pain. Annals of Medicine 1989;21:327-33.

46.Svensson H.O., Andersson G.B.J., Johansson S., Wilhelmsson C., Vedin A. A retrospective study of low-back pain in 38- to 64- year old women. Spine 1988;13(5):548-52.

47.Frymoyer J.W., Pope M.H., Costanza M.C., Rosen J.C., Goggin J.E., Wilder D.G. Epidemiologic studies of low back pain. Spine 1980;5(5):419-23.

48.Clinical Standards Advisory Group. 1994 Epidemiology review: The epidemiology and cost of back pain. HMSO., London. 
49.Walsh K., Cruddas M., Coggon D. Low back pain in eight areas of Britain. Journal of Epidemiology and Community Health 1992;46:227-30.

50.Painting S., Favarin I., SwalesJ. The management of acute industrial low back pain. Physiotherapy 1998;84(3):110-7.

51.Croft P.R., Macfarlane G.J., Papageorgiou A.C., Thomas E., Silman A.J. Outcome of low back pain in general practice: A prospective study. British Medical Journal 1998;316:1356-9.

52.Thomson A. Back pain — the growing epidemic. Physiotherapy 1995;81(2):59-60.

53.Jayson M.I.V. Back pain. British Medical Journal 1996;313:355-8.

54.Spitzer W.O. Low back pain in the workplace: attainable benefits not attained. British Journal of Industrial Medicine 1993;50:385-8.

55.Kelsey J.L. 1982 Idiopathic low back pain: Magnitude of the problem. In: Proceedings of the American Academy of Orthopaedic Surgeons Symposium on low back pain. CV Mosby Co., St. Louis pp5-8.

56.Evans P. The healing process at cellular level: A review. Physiotherapy 1980;66(8):256-9.

57.Waddell G. 1998 The problem. In: The back pain revolution. Churchill Livingstone, Edinburgh pp1-8.

58.Peterson C., Stunkard A.J. Personal control and health promotion. Social Science and Medicine 1989;28(8):819-28.

59.Skevington S.M. 1995 Psychology of pain. J. Wiley and Sons Ltd., Chichester pp13051.

60.Rotter J.B., Chance J.E., Phares E.J. 1972 Applications of a social learning theory of personality. Holt, Rinehart and Winston Inc., New York pp1-43.

61.Arakelian M. An assessment and nursing application of the concept of locus of control. Advances in Nursing Science 1980;3(1):25-42.

62.Smith E.R., Mackie D.M. 1995 Social Psychology. Worth Publishers, New York pp519-23.

63.Rosenstock I.M., Strecher V.J., Becker M.H. Social learning theory and the health belief model. Health Education Quarterly 1988;15(2):175-83.

64.Leventhal H., Benyamini Y., Brownlee S., Diefenbach M., Leventhal E.A., PatrickMiller L., Robitaille C. 1997 Illness representations: theoretical foundations. In: Petrie K.J., Weinman J.A. (eds.) Perceptions of health and illness. Harwood academic publishers, Amsterdam pp19-45.

65.Bonder B.R. Issues in assessment of psychosocial components of function. American Journal of Occupational Therapy 1993;47(3):211-6.

66.Main C.J., Waddell G. 1998 Psychologic distress. In: The back pain revolution. Churchill Livingstone, Edinburgh pp173-87.

67.Ruark J.E. 1990 Death, dying, and grief. In: Wedding D. (ed.) Behaviour and medicine. Mosby, St. Louis pp95-105. 
68.Leventhal E.A., Crouch M. 1997 Are there differences in perceptions of illness across the lifespan. In: Petrie K.J., Weinman J.A. (eds.) Perceptions of health and illness. Harwood academic publishers, Amsterdam pp77-102.

69. Oberle K. A decade of research in locus of control: what have we learned? Journal of Advanced Nursing 1991;16:800-6.

70.Worell L., Tumilty T.N. 1981 The measurement of locus of control among alcoholics. In: Lefcourt H.M. (ed.) Research with the locus of control construct. Volume 1 Assessment methods. Academic Press Inc., New York pp321-33.

71.Strickland B.R. Internal-external expectancies and health-related behaviours. Journal of Consulting and Clinical Psychology 1978;46(6):1192-1211.

72.Paulhus D., Christie R. 1981 Spheres of control: An interactionist approach to assessment of perceived control. In: Lefcourt H.M. (ed.) Research with the locus of control construct. Volume 1 Assessment methods. Academic Press Inc., New York pp161-88.

73.Wallston K.A. Hocus-pocus, the focus isn't strictly on locus: Rotter's social learning theory modified for health. Cognitive Therapy and Research 1992;16(2):183-99.

74.Smith R.E. Changes in locus of control as a function of life crisis resolution. Journal of Abnormal Psychology 1970;75(3):328-32.

75.McCreary C., Turner J. Locus of control, regression-sensitization and psychological disorder in chronic pain patients. Journal of Clinical Psychology 1984;40:897-901.

76.Aasen N. Interventions to facilitate personal control. Journal of Gerontological Nursing 1987;13(6):21-8.

77.Walding M.F. Pain, anxiety and powerlessness. Journal of Advanced Nursing 1991;16:388-97.

78.Wallston K.A., Wallston B.S. 1981 Health locus of control scales. In: Lefcourt H.M. (ed.) Research with the locus of control construct. Volume I Assessment methods. Academic Press Inc., New York pp189-243.

79.Crisson J.E., Keefe F.J. The relationship of locus of control to pain coping strategies and psychological distress in chronic pain patients. Pain 1988;35:147-54.

80.Ponto M.T. Relationship between students' locus of control and satisfaction. British Journal of Nursing 1999;8(3):176-81.

81.Levenson H. 1981 Differentiating among internality, powerful others and chance. In: Lefcourt H.M. (ed.) Research with the locus of control construct. Volume 1 Assessment methods. Academic Press Inc., New York pp15-63.

82.Gregory W.L. 1981 Expectancies for controllability, performance attributions and behaviour. In: Lefcourt H.M. (ed.) Research with the locus of control construct. Volume 1 Assessment methods. Academic Press Inc., New York pp67-124.

83.Calnan M. 1992 Lay beliefs about health and illness. In: French S. (ed.) Physiotherapy. A psychosocial approach Butterworth Heinemann Ltd., Oxford pp244-55. 
84.Wallston B.S., Wallston K.A. Locus of control and health: A review of the literature. Health Education Monographs 1978;6(2):107-17.

85.Bandura A. Self-efficacy mechanism in human agency. American Psychologist 1982a;37(2):122-47.

86.Eiser C., Kopel S.J. 1997 Children's' perceptions of health and illness. In: Petrie K.J., Weinman J.A. (eds.) Perceptions of health and illness Harwood academic publishers, Amsterdam pp47-76.

87.Weisenberg M. 1994 Cognitive aspects of pain. In: Wall P.D., Melzack R. (eds.) Textbook of pain Churchill Livingstone, Edinburgh $3^{\text {rd }}$ edition pp275-89.

88.Strong J. 1995 Self-efficacy and the patient with chronic pain. In: Shacklock M.O. (ed.) Moving in on pain. Butterworth-Heinemann, Australia pp97-102.

89. Tait R., DeGood D., Carron H. A comparison of health locus of control beliefs in low-back patients from the U.S. and New Zealand. Pain 1982;14:53-61.

90.Petrosky M.J., Birkimer J.C. The relationship among locus of control, coping styles and psychological symptom reporting. Journal of Clinical Psychology 1991;47(3):336-45.

91.Härkäpää K., Järvikoski A., Hurri H. Health locus of control beliefs in low back pain patients. Scandinavian Journal of Behaviour Therapy 1989;18:107-18.

92.Buckelew S.P., Shutty M.S., Hewett J., Landon T., Morrow K., Frank R.G. Health locus of control, gender differences and adjustment to persistent pain. Pain 1990;42:287-94.

93.Grace V.M. The marketing of empowerment and the construction of the health consumer: A critique of health promotion. International Journal of Health Services 1991;21(2):329-43.

94.Seeman M., Evans J.W. Alienation and learning in a hospital setting. American Sociological Review 1962;27:772-83.

95.Johnson L.R., Magnani B., Chan V., Ferrante F.M. Modifiers of patient-controlled analgesia efficacy. 1: Locus of control. Pain 1989;39:17-22.

96.Skevington S.M. 1979 Pain and locus of control - a social approach. In: Osborne D.J., Gruneberg M.M., Eiser J.R. (eds.) Research in psychology and medicine. Volume 1. Academic Press, London pp61-9.

97.Montbriand M.J., Liang G.P. Alternative health care as a control strategy. Journal of Advanced Nursing 1991;16:325-32.

98.Waddell G., Main C.J. 1998 Beliefs about back pain. In: The back pain revolution. Churchill Livingstone, Edinburgh pp187-202.

99.Horne R. 1997 Representations of medication and treatment: Advances in theory and measurement. In: Petrie K.J., Weinman J.A. (eds.) Perceptions of health and illness. Harwood academic publishers, Amsterdam pp155-88.

100.Waddell G., Main C.J. 1998 A new clinical model of low back pain and disability. In: The back pain revolution. Churchill Livingstone, Edinburgh pp223-240. 
101.Callery P. Moral learning in nursing education: a discussion of the usefulness of cognitive-developmental and social learning theories. Journal of Advanced Nursing 1990;15:324-8.

\section{Chapter 3: G.P. questionnaire}

1. Clinical Standards Advisory Group. 1994 Back pain. Report of a CSAG committee on back pain. HMSO., London.

2. Deyo R.A. Low-back pain. Scientific American Aug. 1998:28-33.

3. Cherkin D.C., Deyo R.A., Wheeler K., Ciol M.A. Physician variation in diagnostic testing for low back pain. Who you see is what you get. Arthritis and Rheumatism 1994; 37(1):15-22.

4. Walsh K. 1992 An epidemiological study of low back pain. Doctoral Thesis, University of Southampton, Faculty of Medicine.

5. Mason V. 1994 The prevalence of back pain in Great Britain. A report on OPCS Omnibus Survey data produced on behalf of the Department of Health. HMSO., London pp9.

6. Ingham J.G., Miller P. McC. Self-referral to primary care: symptoms and social factors. Journal of Psychosomatic Research 1986;30(1):49-56.

7. Cameron L., Leventhal E.A., Leventhal H. Seeking medical care in response to symptoms and life stress. Psychosomatic Medicine 1995;57:37-47.

8. Wilson A., McDonald P., Hayes L., Cooney J. Health promotion in the general practice consultation: a minute makes a difference. British Medical Journal 1992;304:227-30.

9. Little P., Williamson I., Warner G., Gould C., Gantley M., Kinmonth A.L. Open randomised trial of prescribing strategies in managing sore throat. British Medical Journal 1997;314:722-7.

10. Briscoe M.E. Why do people go to the doctor? Sex differences in the correlates of GP consultation. Social Science and Medicine 1987;25(5):507-13.

11.Walsh K., Cruddas M., Coggon D. Low back pain in eight areas of Britain. Journal of Epidemiology and Community Health 1992;46:227-30.

12.Grace V.M. The marketing of empowerment and the construction of the health consumer: A critique of health promotion. International Journal of Health Services 1991;21(2):329-43.

13. Skelton R. Nursing and empowerment: Concepts and strategies. Journal of Advanced Nursing 1994;19:415-23.

14. McKay B., Forbes J., Bourner K. Empowerment in general practice. The trilogies of caring. Australian Family Physician 1990;19(4):513-20.

15. Clifton B. Overpowering empowerment. Nursing Standard 1993;7(31):45.

16. Troop N., Treasure J., Schmidt U. From specialist care to self directed treatment. Empower the patient and spare the clinician. British Medical Journal 1993;307:5778. 
17. Caraher M. Patient education and health promotion: Clinical health promotion - the conceptual link. Patient Education and Counseling 1998;33:49-58.

18. Mayberry J.F., Mayberry M.K. Effective instructions for patients. Journal of the Royal College of Physicians of London 1996;30(3):205-8.

19. Skelton A.M., Murphy E.A., Murphy R.J.L., O'Dowd T.C. Patient education for low back pain in general practice. Patient Education and Counseling 1995;25:329-34.

20. Streiner D.L., Norman G.R. 1989 Reliability. In: Health measurement scales. A practical guide to their development and use. Oxford University Press, Oxford pp7996

21. Stephenson B. Personal communication 1994.

22. Little P, Smith L, Cantrell T, Chapman J, Langridge J, Pickering R. General practitioners' management of acute back pain: a survey of reported practice compared with clinical guidelines. British Medical Journal 1996;312:485-8.

23. Krilyk J., Eyles P. Personal communication 1994. McMaster University, Hamilton Ontario.

24. Williams P., Warwick R. (eds.) 1986 Gray's Anatomy. Churchill Livingstone, Edinburgh 36th edition p1407.

25. Bickerstaff E. 1973 Neurological Examination in Clinical Practice. Blackwell Scientific Publications, 199-200.

26. O'Reilly S. The extensor plantar response. Irish Medical Journal 1992;85(2):49.

27. Walshe F. The Babinski plantar response, its forms and its physiological and pathological significance. Brain 1956;79:529-56.

28. Grant R. The neurological assault on the great toe (1893-1911). Scottish Medical Journal 1987;32:57-59.

29. MacGregor J.M. That up-going toe. South African Medical Journal 1987;71(9):5924.

30. Maher J., Reilly M., Daly L., Hutchinson M. Plantar power: reproducibility of the plantar response. British Medical Journal 1992;304:482.

31. Mayo Clinic and Mayo Foundation. 1971 Clinical Examinations in Neurology. Philadelphia: W.B. Saunders Co., 175.

32. Guyer R., Collier R., Ohnmeiss D., Stith W., Hochschuler S., Rashbaum R., Vanharanta $H$., Loguidice V. Extraosseous spinal disease mimicking disc disease. Spine 1988;13(3):328-31.

33. Levy W.J., Latchaw J., Hahn J.F., Sawhny B., Bay J., Dohn D.F. Spinal neurofibromas: a report of 66 cases and a comparison with meningiomas. Neurosurgery 1986;18(3):331-4.

34. Delamarter R.B., Sachs B.L., Thompson G.H., Bohlman H.H., Makley J.T., Carter J.R. Primary neoplasms of the thoracic and lumbar spine. An analysis of 29 consecutive cases. Clinical Orthopaedics and Related Research 1990;(256):87-100. 
35. Nayernouri T. Neurilemomas of the cauda equina presenting as prolapsed lumbar intervertebral disks. Surgical Neurology 1985;23:187-8.

36. Donaldson W.F., Peppelman W.C., Yaw K.M. Symptomatic metastatic malignant melanoma to the spine. Journal of Spinal Disorders 1993;6(4):360-3.

37. Paterson J., Burn L. 1986 Examination of the back. Lancaster : MTP Press Limited, 62,91 .

38. Grieve G. Mobilisation of the spine. 1991 Churchill Livingstone Edinburgh pp53, 171.

39. Dinning T.A.R., Schaeffer H.R. Discogenic compression of the cauda equina: a surgical emergency. Australian and New Zealand Journal of Surgery 1993;63:92734.

40.Deyo R.A. Early diagnostic evaluation of low back pain. Journal of General Internal Medicine 1986;1:328-38.

41. Matthew P., Todd N. Diagnosis of intradural conus and cauda equina tumours. British Journal of Hospital Medicine 1993;50(4):169-74.

42. Boriani S., Capanna R., Donati D., Levine A., Picci P., Savini R. Osteoblastoma of the spine. Clinical Orthopaedics and Related Research 1992;278:37-45.

43. Evans G., Richards S. 1996 Low back pain: an evaluation of therapeutic interventions. Health Care Evaluation Unit, University of Bristol pp28-32.

44. RCR Working Party. 1995 Making the best use of a Department of Clinical Radiology. Guidelines for Doctors. The Royal College of Radiologists, London $3^{\text {rd }}$ edition.

45. RCR Working Party. 1998 Making the best use of a Department of Clinical Radiology. Guidelines for Doctors. The Royal College of Radiologists, London $4^{\text {th }}$ edition.

46. RCR Working Party. 1993 Making the best use of a Department of Clinical Radiology. Guidelines for Doctors. The Royal College of Radiologists, London $2^{\text {nd }}$ edition.

47. Agency for Health Care Policy and Research. 1994 Management guidelines for acute low back pain. U.S. Department of Health and Human Services, Rockville MD.

48.Spitzer W.O., LeBlanc F.E., Dupuis M. et al. Scientific approach to the assessment and management of activity-related spinal disorders. Spine 1987;12(7S):S1-S59.

49. Waddell G., Feder G., McIntosh A., Lewis M., Hutchinson A. 1996. Low back pain evidence review. London: Royal College of General Practitioners. pp l-34.

50. Rogers L.F. 1993 Bone tumors and related conditions. In: Juhl J.H., Crummy J.B. (eds.) Essentials of radiologic images. Lippincott pp139-86.

51. A.C.C. and the National Health Committee 1997. New Zealand acute low back pain guide. Wellington, NZ. pp1-13.

52. Halpin S.F.S., Yeoman L., Dundas D.D. Radiographic examination of the lumbar spine in a community hospital: an audit of current practice. British Medical Journal 1991;303:813-5. 
53. Klaber Moffett J., Richardson G., Sheldon T.A., Maynard A. 1995 Back pain. Its management and cost to society. Discussion paper 129, Centre for Health Economics, University of York pp20.

54. Bogduk N. 1994 Innervation, Pain Patterns and mechanisms of Pain Production. In: Twomey L., Taylor J. (eds.) Clinics in Physical therapy of the Low Back. Churchill Livingstone $2^{\text {nd }}$ edition pp93-109.

55. Buchanan M. Enabling patients to make informed decisions. Nursing Times 1995;91(18):27-9.

56. Roland M., Waddell G., Klaber Moffett J., Burton K., Main C., Cantrell T. 1996 The Back Book. The Stationery Office Ltd., Norwich pp1-23.

57. National Bed Federation. 'You'll feel better with a new bed'. Healthcare 1992; spring issue:26-7.

58. National Back Pain Association. Better backs for drivers leaflet

59. Hides J.A., Richardson C.A., Jull G.A. Multifidus muscle recovery is not automatic after resolution of acute, first-episode low back pain. Spine 1996;21(23):2763-9.

60. Frost H., Klaber Moffett J.A., Moser J.S., Fairbank J.C.T. Randomised controlled trial for evaluation of fitness programme for patients with chronic low back pain. British Medical Journal 1995;310:151-4.

61. Nachemson A.L. The lumbar spine. An orthopaedic challenge. Spine 1976;1(1):5971.

62. National Back Pain Association. Better backs for gardeners leaflet

63. Bed rest for back pain (leaflet). In: 'Diagnosis of the prolapsed intervertebral disc, conservative management and further treatment options'. Boots Pharmaceuticals U.K., Nottingham 1993

64. Rowling J., Roberts L. The use of acupuncture by chartered physiotherapists in the United Kingdom: A review of undergraduate students' attitudes and knowledge. Physical Therapy Reviews 1999;4:97-103.

65. Little P., Cantrell T., Roberts L., Chapman J., Langridge J., Pickering R. Why do GPs perform investigations?: the medical and social agendas in arranging back X-rays. Family Practice 1998;15(3):264-5.

\section{Chapter 4: Measurement}

1. Guyatt G., Feeny D., Patrick D. Measuring health-related quality of life. Annals of Internal Medicine 1993;118(8):622-9.

2. Chapman C.R. 1976 Measurement of pain: problems and issues. In: Bonica J.J., Albe-Fessard D. (eds.) Advances in Pain Research and Therapy. Vol. I. New York: Raven Press pp345-53.

3. Nelson E.C., Berwick D.M. The measurement of health status in clinical practice. Medical Care 1989;27(3):Supplement S77-90.

4. Task Force. Standards for tests and measurements in physical therapy practice. Physical Therapy 1991;71(8):589-622. 
5. Neuberger J. The public face of outcomes. Health Service Journal 1993;25 ${ }^{\text {th }}$ Feb:19.

6. Brooks R.G. 1995 The upsurge of interest in health status measurement. In: Health status measurement: A perspective on change. MacMillan Press Ltd., Basingstoke pp1-14.

7. Weisenberg M. 1994 Cognitive aspects of pain. In: Wall P.D., Melzack R. (eds.) Textbook of pain. Churchill Livingstone, Edinburgh $3^{\text {rd }}$ edition pp275-89.

8. Blaxter M. 1990 Introduction. In: Health and lifestyles. Routledge, London pp1-8.

9. Blaxter M. 1990 What is health? In: Health and lifestyles. Routledge, London pp1334.

10. Gill T.M., Feinstein A.R. A critical appraisal of the quality of quality-of-life measurements. Journal of the American Medical Association 1994;272(8):619-26.

11. Hirsch G., Beach G., Cooke C., Menard M., Locke S. Relationship between performance on lumbar dynamometry and Waddell score in a population with low back pain. Spine 1991;16(9):1039-43.

12. Snaith R.P. Bridge G.W.K., Hamilton M. The Leeds Scales for the assessment of anxiety and depression. British Journal of Psychiatry 1976;128:156-65.

13. Delitto A. Are measures of function and disability important in low back care? Physical Therapy 1994;74:452-62.

14. Parkerson G.R., Deyo R.A., Golden W.E., Blim R.D., Patrick D.L., Tuteur P.G., et al. Strategies for improving and expanding the application of health status measures in clinical settings. Medical Care 1992;30(5):Supplement MS210-8.

15. Guyatt G.H., Kirshner B., Jaeschke R. A Methodologic Framework for Health Status Measures: Clarity or Oversimplification? Journal of Clinical Epidemiology 1992;45(12):1353-5.

16. Deyo R.A., Tsui-Wu Y.J. Descriptive Epidemiology of Low-Back Pain and its Related Medical Care in the United States. Spine 1987; 12(3):264-8.

17.Bonder B.R. Issues in assessment of psychosocial components of function. American Journal of Occupational Therapy 1993;47(3):211-6.

18. Waddell G. A new clinical model for the treatment of low-back pain. Spine $1987 ; 12(7): 632-44$.

19.Guyatt G.H., Kirshner B., Jaeschke R. Measuring health status: What are the necessary measurement properties? Journal Clinical Epidemiology 1992;45(12):13415.

20. McDowell I., Newell C. 1987 Measuring health: a guide to rating scales and questionnaires. Oxford University Press, New York.

21. Law M. Measurement in occupational therapy: scientific criteria for evaluation. Canadian Journal of Occupational Therapy 1987;54(3):133-8.

22. Bombardier C., Tugwell P. Methodological consideration in functional assessment. Journal of Rheumatology 1987;14(supplement 15):6-10. 
23. Streiner D.L., Norman G.R. 1989 Health measurement scales. A practical guide to their development and use. Oxford University Press, Oxford.

24. Brooks R.G. 1995 An outline of the development of health status measures. In: Health status measurement: A perspective on change. MacMillan Press Ltd., Basingstoke pp15-44.

25. World Health Organization. 1980 International Classification of Impairments, Disabilities and Handicaps. Geneva, Switzerland.

26. Guccione A.A. Physical therapy diagnosis and the relationship between impairments and function. Physical Therapy 1991;71:499-504.

27. Streiner D.L., Norman G.R. 1989 Basic concepts. In: Health measurement scales. $A$ practical guide to their development and use. Oxford University Press, Oxford pp410.

28. Chambers L.W. Physical and emotional function of primary care patients: Scientific requirements for the measurement of functional health status. Journal of the Americal Medical Association 1983;249(24):3353-5.

29. Brooks R.G. 1995 Methodology. In: Health status measurement: A perspective on change. MacMillan Press Ltd., Basingstoke pp45-56.

30. Cole B., Finch E., Gowland C., Mayo N. 1994 Physical rehabilitation outcome measures. Canadian Physiotherapy Association, Toronto pp21-33.

31. Streiner D.L., Norman G.R. 1989 Reliability. In: Health measurement scales. A practical guide to their development and use. Oxford University Press, Oxford pp7996.

32. Adams M.A., Dolan P., Hutton W.C., Porter R.W. Diurnal changes in spinal mechanics and their clinical significance. Journal of Bone and Joint Surgery 1990;72(2):266-70.

33. Streiner D.L., Norman G.R. 1989 Selecting the items. In: Health measurement scales. A practical guide to their development and use. Oxford University Press, Oxford pp39-53.

34.Spitzer W.O., LeBlanc F.E., Dupuis M. et al. Scientific approach to the assessment and management of activity-related spinal disorders. Spine 1987;12(7S):S1-S59.

35.Croft P.R., Macfarlane G.J., Papageorgiou A.C., Thomas E., Silman A.J. Outcome of low back pain in general practice: A prospective study. British Medical Journal 1998;316:1356-9.

36. Waddell G., Main C. Assessment of severity in low-back disorders. Spine 1984;9(2):204-8.

37. Chartered Society of Physiotherapy. Standards for administering tests and taking measurements for chartered physiotherapists. Chartered Society of Physiotherapy pp1-37.

38. Streiner D.L., Norman G.R. 1989 Validity. In: Health measurement scales. A practical guide to their development and use. Oxford University Press, Oxford pp106-25. 
39. Johnston M.V., Keith R.A., Hinderer S.R. Measurement standards for interdisciplinary medical rehabilitation. Archives of Physical Medicine and Rehabilitation 1992;73:S3-S23.

40. Streiner D.L., Norman G.R. 1989 Measuring change. In: Health measurement scales. A practical guide to their development and use. Oxford University Press, Oxford pp126-37.

41. Kirshner B., Guyatt G. A methodological framework for assessing health indices. Journal of Chronic Diseases 1985;38(1):27-36.

42. Bowling A. 1989 Measuring disease. A review of disease-specific quality of life measurement scales. Open University Press, Buckingham.

43. Rotter J.B. Some problems and misconceptions related to the construct of internal versus external control of reinforcement. Journal of Consulting and Clinical Psychology 1975;43(1):56-67.

44. Wallston K.A., Wallston B.S., DeVellis R. Development of the multidimensional health locus of control (M.H.L.C.) scales. Health Education Monographs 1978;6(2):160-70.

45. Bradley C., Brewin C.R., Gamsu D.S., Moses J.L. Development of scales to measure perceived control of diabetes mellitus and diabetes-related health beliefs. Diabetic Medicine 1984;1:213-8.

46. Winefield H.R. Reliability and validity of the health locus of control scale. Journal of Personality Assessment 1982;46(6):614-9.

47. Keith R.A. Functional status and health status. Archives of Physical Medicine and Rehabilitation 1994;75:478-83.

48. Greenough C.G., Fraser R.D. Assessment of outcome in patients with low back pain. Spine 1992;17(1):36-41.

49. Fairbank J.C.T., Couper J., Davies J.B., O’Brien J.P. The Oswestry low back pain disability questionnaire. Physiotherapy 1980;66(8):271-3.

50. Roland M., Morris R. A study of the natural history of back pain - part 1: Development of a reliable and sensitive measure of disability in low back pain. Spine 1983;8(2):141-4.

51. Ware J.E., Sherbourne C.D. The M.O.S. 36-item short-form health survey (SF-36). Medical Care 1992;30:473-8.

52. Jenkinson C. Coulter A., Wright L. Short form 36 health survey questionnaire: Normative data for adults of working age. British Medical Journal 1993;306:143740.

53. Garratt A.M., Ruta D.A., Abdalla M.I., Buckingham J.K., Russell I.T. The SF36 health survey questionnaire: An outcome measure suitable for routine use within the N.H.S. British Medical Journal 1993;306:1440-4.

54. Brazier J.E. Validating the SF-36 health survey questionnaire: A new outcome measure for primary care. British Medical Journal 1992;305:160-4. 
55. Bergner M., Bobbitt R.A., Carter W.B., Gilson B.S. The sickness impact profile: development and final revision of a health status measure. Medical Care 1981;19(8):787-805.

56. Bergner M., Bobbitt R.A., Kressel S., Pollard W.E., Gilson B.S., Morris J.R. The Sickness Impact Profile: conceptual formulation and methodology for the development of a health status measure. International Journal Health Service 1976;6(3):393-415.

57. deBruin A.F., deWitte L.P., Diedriks J. Sickness Impact Profile: the state of the art of a generic functional status measure. Social Science and Medicine 1992;35(8):1003-14.

58. Hunt S.M., McKenna S.P., McEwen J., Backett E.M., Williams J., Papp E. A quantitative approach to perceived health status: a validation study. Journal of Epidemiology and Community Health 1980;34(4):281-6.

59. Kind P., Carr-Hill R. The Nottingham Health Profile: a useful tool for epidemiologists? Social Science and Medicine 1987;25:905-10.

60. Hunt S., McEwen J., McKenna S.P. Measuring health status: a new tool for clinicians and epidemiologists. Journal of the Royal College of General Practitioners 1985;35(273):185-8.

61. Hunt S.M., McKenna S.P., McEwen J., Williams J., Papp E. The Nottingham Health Profile: subjective health status and medical consultations. Social Science and Medicine 1981;15(A):221-9.

62. Ruta D.A., Garratt A., Wardlaw D., Russell I. Developing a valid and reliable measure of health outcome for patients with low back pain. Spine 1994;19(17):1887-96.

63. Pynsent P., Fairbank J., Carr A. 1993 Outcome measures in orthopaedics. Butterworth-Heinemann Ltd., Oxford.

64. Cole B., Finch E., Gowland C., Mayo N. 1994 Back and/or pain measures. In: Physical rehabilitation outcome measures. Canadian Physiotherapy Association, Toronto pp80-91.

65. LeClaire R., Blier F., Fortin L., Proulx R. A cross-sectional study comparing the Oswestry and Roland-Morris functional disability scales in two populations of patients with low back pain of different levels of severity. Spine 1997;22(1):68-71.

66. Anderson R.T., Aaronson N.K., Wilkin D. Critical review of the international assessments of health-related quality of life. Quality of life research 1993;2:369-95.

67. Bowling A. 1991 Measuring health. A review of quality of life measurement scales. Open University Press, Milton Keynes pp58-70.

68. Deyo R.A. Comparative validity of the sickness impact profile and shorter scales for functional assessment in low-back pain. Spine 1986;11(9):951-4.

69. Reynolds W.J., Rushing W.A., Miles D.L. The validation of a functional status index. Journal of health and social behaviour 1974;15:271-83.

70. Read J.L., Quinn R.J., Hoefer M.A. Measuring overall health: an evaluation of three important approaches. Journal of Chronic Diseases 1987;40 suppl.1:7S-21S. 
71. Pollard W.E., Bobbitt R.A., Bergner M., Martin D.P., Gilson B.S. The Sickness Impact Profile: Reliability of a health status measure. Medical Care 1976;14(2):14655.

72. Sim J., Waterfield J. Validity, reliability and responsiveness in the assessment of pain. Physiotherapy Theory and Practice 1997;13:23-37.

73. Kremer E., Atkinson J.H., Ignelzi R.J. Measurement of pain: patient preference does not confound pain measurement. Pain 1981;10(2):241-8.

74. Huskisson E. Measurement of pain. The Journal of Rheumatology 1982;9(5):768-9.

75. Rainville J., Ahern D.K., Phalen L., Childs L.A., Sutherland R. The association of pain with physical activities in chronic low back pain. Spine 1992;17(9):1060-4.

76. Melzack R. The McGill Pain Questionnaire: major properties and scoring methods. Pain 1975;1:277-99.

77. Wilkie D.J., Savedra M.C., Holzemer W.L., Tesler M.D., Paul S.M. Use of the McGill Pain Questionnaire to measure pain: a meta-analysis. Nursing Research 1990;39(1):36-41.

78. Scott J., Huskisson E. Graphic representation of pain. Pain 1976;2:175-84.

79. Huskisson E.C. Measurement of pain. The Lancet 1974;Nov. $9^{\text {th }} 2$ (7889):1127-31.

80. Scott J., Huskisson E.C. Vertical or horizontal visual analogue scales. Annals of the Rheumatic Diseases 1979;38:560.

81. Downie W.W., Leatham P.A., Rhind V.M., Wright V., Branco J.A., Anderson J.A. Studies with pain rating scales. Annals of the Rheumatic Diseases 1978;37(4):378-81.

82. Linton S.J., Götestam K.G. A clinical comparison of two pain scales: correlation, remembering chronic pain and a measure of compliance. Pain 1983;17:57-65.

83. Ohnhaus E.E., Adler R. Methodological problems in the measurement of pain: a comparison between the verbal rating scale and the visual analogue scale. Pain 1975;1:379-84.

84. Machin D., Lewith G.T., Wylson S. Pain measurement in randomized clinical trials. The Clinical Journal of Pain 1988;4:161-8.

85. Melzack R., Torgerson W.S. On the language of pain. Anaesthesiology 1971;34(1):50-9.

86. Melzack R. The short-form McGill Pain Questionnaire. Pain 1987;30:191-7.

87. Woodforde J.M., Merskey H. Some relationships between subjective measures of pain. Journal of Psychosomatic Research 1972;16:173-8.

88. Sriwatanakul K., Kelvie W., Lasagna L., Calimlim J.F., Weis O.F., Mehta G. Studies with different types of visual analog scales. Clinical Pharmacology and Therapeutics 1983;34(2):234-9.

89. Jensen M.P., Karoly P., Braver S. The measurement of clinical pain intensity: a comparison of six methods. Pain 1986;27:117-26. 
90. Streiner D.L., Norman G.R. 1989 Scaling responses. In: Health measurement scales. A practical guide to their development and use. Oxford University Press, Oxford pp20-38.

91.Waddell G. 1998 Pain and disability. In: The back pain revolution. Churchill Livingstone, Edinburgh pp27-44.

92. Spielberger C.D. Anxiety and Behaviour. (ed). Spielberger C.D. Academic Press, New York 1966 ppl2-9.

93. Spielberger C.D. Manual for the State-Trait Anxiety Inventory.

94. Marteau T.M., Bekker H. The development of a six-item short-form of the state scale of the Spielberger State-Trait Anxiety Inventory (S.T.A.I.) British Journal of Clinical Psychology 1992;31:301-6.

95. Zigmond A.S., Snaith R.P. The Hospital Anxiety and Depression Scale. Acta Psychiatr. Scandinavica 1983;67:361-70.

96. Powell T.J., Enright T.J. 1990 A useful battery of anxiety questionnaires. In: Anxiety and stress management. Routledge, London pp57-63.

97. Houtman I.L.D., Bakker F.C. The Anxiety Thermometer. Journal of Personality Assessment 1989;53(3):575-82.

98. Zuckerman M., Lubin B., Vogel L., Valerius E. Measurement of experimentally induced affects. Journal of Consulting Psychology 1964;28(5):418-25.

99. Lader M., Marks I. 1971 The measurement of anxiety. Clinical Anxiety Heinemann Ltd., London pp82-108.

100.Herrmann C. International experiences with the $\mathrm{HAD}$ Scale - a review of validation data and clinical results. Journal of Psychosomatic Research 1997;42(1):17-41.

101.Snaith R.P., Zigmond A.S. The hospital anxiety and depression scale (letter). British Medical Journal 1986;292:344.

102.Partridge C., Johnston M. Perceived control of recovery from physical disability: Measurement and prediction. British Journal of Clinical Psychology 1989;28:53-9.

103.Brooks R.G. 1995 Trends and issues. In: Health status measurement: A perspective on change. MacMillan Press Ltd., Basingstoke pp115-21.

104.Coxhead C.E., Inskip H., Meade T.W., North W.R.S., Troup J.D.G. Multicentre trial of physiotherapy in the management of sciatic symptoms. The Lancet 1981;May 16:1065-8.

\section{Chapter 5: The 'Back Home' study}

1. Donovan J.L., Blake D.R., Fleming W.G. The patient is not a blank sheet: lay beliefs and their relevance to patient education. British Journal of Rheumatology 1989;28:58-61.

2. Smith C.E. Overview of patient education. Opportunities and challenges for the twenty-first century. Nursing Clinics of North America 1989;24(3):583-7. 
3. Daltroy L.H., Liang M.H. Advances in patient education in rheumatic disease. Annals of the Rheumatic Diseases 1991;50:415-7.

4. Redman B.K. Patient education as a function of nursing practice. Nursing Clinics of North America 1971;6(4):573-80.

5. Close A. Patient education: A literature review. Journal of Advanced Nursing 1988;13:203-13.

6. Chartered Society of Physiotherapy. 1996 Rules of professional conduct. Chartered Society of Physiotherapy, London.

7. Ethics Committee. 1995 Code of ethics and professional conduct for Occupational Therapists. College of Occupational Therapists, London.

8. American Physical Therapy Association. 1995 Guidelines for physical therapy documentation. American Physical Therapy Association, Alexandria U.S.A.

9. Beisecker A.E., Beisecker T.D. Patient information-seeking behaviors when communicating with doctors. Medical Care 1990;28(1):19-28.

10.Kipling R. I keep six honest serving-men.

11.Harland C.C., Madeley R.J., Millard L.G. Information leaflets in the dermatology out-patient waiting area. British Journal of Dermatology 1992;127:492-6.

12.Luker K., Caress A.L. Rethinking patient education. Journal of Advanced Nursing 1989;14:711-8.

13.Cook T. Major research analysis provides proof: Patient education does make a difference. Promoting Health 1984:4-9.

14.Troop N., Treasure J., Schmidt U. From specialist care to self directed treatment. British Medical Journal 1993;307:577-8.

15.Cherkin D.C., Deyo R.A., Street J.H., Hunt M., Barlow W. Pitfalls of patient education. Limited success of a program for back pain in primary care. Spine 1996;21(3):345-55.

16.Nordin M. Back pain: lessons form patient education. Patient Education and Counseling 1995;26:67-70.

17.Tucker M., Kirwan J.R. Does patient education in rheumatoid arthritis have therapeutic potential? Annals of the Rheumatic Diseases 1991;50:422-8.

18.Cupples S.A. Effects of timing and reinforcement of preoperative education on knowledge and recovery of patients having coronary artery bypass graft surgery. Heart \& Lung 1991;20(6):654-60.

19.Donovan J. Patient education and the consultation: the importance of lay beliefs. Annals of the Rheumatic Diseases 1991;50:418-21.

20.Savage S.A., Hollin C.R., Hayward A.J. (in Troop). Self-help manuals for problem drinking: the relative effects of their educational and therapeutic components. British Journal of Clinical Psychology 1990;20:373-82.

21.Roux E. Developing an educational book about back pain: problems encountered possible solutions. Patient Education and Counseling 1995;26:363-6. 
22.Livesley P.J., Rider M.A. Joint replacement and patient education. International Orthopaedics 1993;17(1):34-6.

23.Moll J.M.H. Doctor-patient communication in rheumatology: Studies of visual and verbal perception using educational booklets and other graphic material. Annals of the Rheumatic Diseases 1986;45:198-209.

24.Hadler N.M. Point of view. Spine 1996;21(3):355.

25.Roland M, Dixon M. Randomized controlled trial of an educational booklet for patients presenting with back pain in general practice. Journal of the Royal College of General Practitioners 1989;244-6.

26.Burton K., Tillotson M., Symonds T., Sykes D. 1993 Back pain. Don't suffer needlessly. (leaflet) Spinal Research Unit, University of Huddersfield.

27.Roland M., Dixon M. 1995 The Back Book. Your guide to back care. (leaflet).

28.Chapman J., Langridge J. Physiotherapy health education literature. Physiotherapy 1997;83(8):406-12.

29. Krilyk J., Eyles P. Personal communication 1994. McMaster University, Hamilton Ontario.

30.Marteau T., Bekker H. The 6-item Short Form of the State Scale of the Spielberger State-Trait Anxiety Inventory. British Journal of Clinical Psychology 1992;31:301-6.

31.Ruta D., Garratt A., Wardlaw D., Russell I. Developing a valid and reliable measure of health outcome for patients with low back pain. Spine 1994;19(17):1887-96.

32.Jessep S. Comparison study of the effectiveness of two methods of back education: a pilot study. New Zealand Journal of Physiotherapy 1991;19(3):32-9.

33.Wallston K., Wallston B., DeVellis R. Development of the Multidimensional Health Locus of Control Scales. Health Education Monographs 1978;6(2):160-70.

34.Melzack R. The McGill Pain Questionnaire: major properties and scoring methods. Pain 1975;1:277-99.

35. Bradley C., Brewin C.R., Gamsu D.S., Moses J.L. Development of scales to measure perceived control of diabetes mellitus and diabetes-related health beliefs. Diabetic Medicine 1984;1:213-8.

36. Streiner D.L., Norman G.R. 1989 Reliability. In: Health measurement scales. $A$ practical guide to their development and use. Oxford University Press, Oxford pp7996.

37. Nachemson A.L. The lumbar spine. An orthopaedic challenge. Spine 1976;1(1):5971.

38. Moser J. 1990 An investigation into changes in knowledge and behaviour following attendance at a Backschool. M.Sc. Dissertation, University of Southampton, Faculty of Medicine. 


\section{Chapter 6: Validation of the M.H.L.C. form C}

1. Wallston K.A., Wallston B.S., DeVellis R. Development of the multidimensional health locus of control (M.H.L.C.) scales. Health Education Monographs 1978;6(2):160-70.

2. Shakespeare W. Julius Caesar. Act 4 Scene 3.

3. French S. 1993 Practical research. A guide for therapists. Butterworth Heinemann Ltd., Oxford pp11-2.

\section{Chapter 7: Control issues}

1. Wallston K.A. Personal communication (see appendix 6.1)

2. Norusis M.J. 1990 The S.P.S.S. guide to data analysis for release 4. SPSS Inc., Chicago pp143-6.

3. Härkäpää K., Järvikoski A., Hurri H. Health locus of control beliefs in low back pain patients. Scandinavian Journal of Behaviour Therapy 1989;18:107-18.

4. Tait R., DeGood D., Carron H. A comparison of health locus of control beliefs in low -back patients from the US and New Zealand. Pain 1982;14:53-61.

5. Smith R.E. Changes in locus of control as a function of life crisis resolution. Journal of Abnormal Psychology 1970;75(3):328-32.

6. Marteau T., Bekker H. The 6-item Short Form of the State Scale of the Spielberger State-Trait Anxiety Inventory. British Journal of Clinical Psychology 1992;31:301-6.

7. Hicks C.M. 1995 Research for physiotherapists. Project design and analysis. Churchill Livingstone, Edinburgh 2nd edition pp79-87, 119-146.

\section{Chapter 8: Follow-up G.P. questionnaire}

1. Clinical Standards Advisory Group. 1994 Back pain. Report of a CSAG committee on back pain. HMSO., London.

2. Waddell G., Feder G., McIntosh A., Lewis M., Hutchinson A. 1996. Low back pain evidence review. London: Royal College of General Practitioners. pp1-34.

3. Oppenheim A.N. 1992. Questionnaire design, interviewing and attitude measurement. Pinter Publishers Ltd London p102-4.

4. Robson C. 1993 Real world research. Blackwell Publishers, Oxford. p125.

5. Rudy E.B., Estok P.J., Kerr M.E., Menzel L. Research incentives: money versus gifts. Nursing Research. 1994;43(4):253-5.

\section{Chapter 9: Overview}

1. Spitzer W.O., LeBlanc F.E., Dupuis M. et al. Scientific approach to the assessment and management of activity-related spinal disorders. Spine 1987;12(7S):S1-S59.

2. Clinical Standards Advisory Group. 1994 Back pain. Report of a CSAG committee on back pain. HMSO., London. 
3. Croft P.R., Macfarlane G.J., Papageorgiou A.C., Thomas E., Silman A.J. Outcome of low back pain in general practice: A prospective study. British Medical Journal 1998;316:1356-9.

4. Wallston K.A. Hocus-pocus, the focus isn't strictly on locus: Rotter's social learning theory modified for health. Cognitive Therapy and Research 1992;16(2):183-99.

5. Task Force. Standards for tests and measurements in physical therapy practice. Physical Therapy 1991;71(8):589-622.

6. Walsh K., Cruddas M., Coggon D. Low back pain in eight areas of Britain. Journal of Epidemiology and Community Health 1992;46:227-30.

7. Moser J. 1990 An investigation into changes in knowledge and behaviour following attendance at a Backschool. M.Sc. Dissertation, University of Southampton, Faculty of Medicine.

8. Harland C.C., Madeley R.J., Millard L.G. Information leaflets in the dermatology out-patient waiting area. British Journal of Dermatology 1992;127:492-6.

9. Hadler N.M. Point of view. Spine 1996;21(3):355.

10. Little P, Smith L, Cantrell T, Chapman J, Langridge J, Pickering R. General practitioners' management of acute back pain: a survey of reported practice compared with clinical guidelines. British Medical Journal 1996;312:485-8.

11.Waddell G, Feder G, McIntosh A, Lewis M, Hutchinson A 1996. Low back pain evidence review. London: Royal College of General Practitioners pp1-34.

12.Sedaghat M. 1998 An investigation into what expectations people with low back pain have before they attend a Backschool and whether their expectations were met by attending a Backschool. Undergraduate dissertation, University of Southampton.

13.Prior J. 1999 An investigation into the knowledge and attitudes of undergraduate second year physiotherapy students towards Backschools. Undergraduate dissertation, University of Southampton.

14.Rothman C. 1997 How can physiotherapy meet the needs of people with chronic low back pain in an outpatient department? An investigative study. MSc Health Psychology dissertation, University of Southampton.

15.A.C.C. and the National Health Committee 1997. New Zealand acute low back pain guide. Wellington, NZ. pp1-13.

16.Shakespeare W. Macbeth Act 5 Scene 1. 


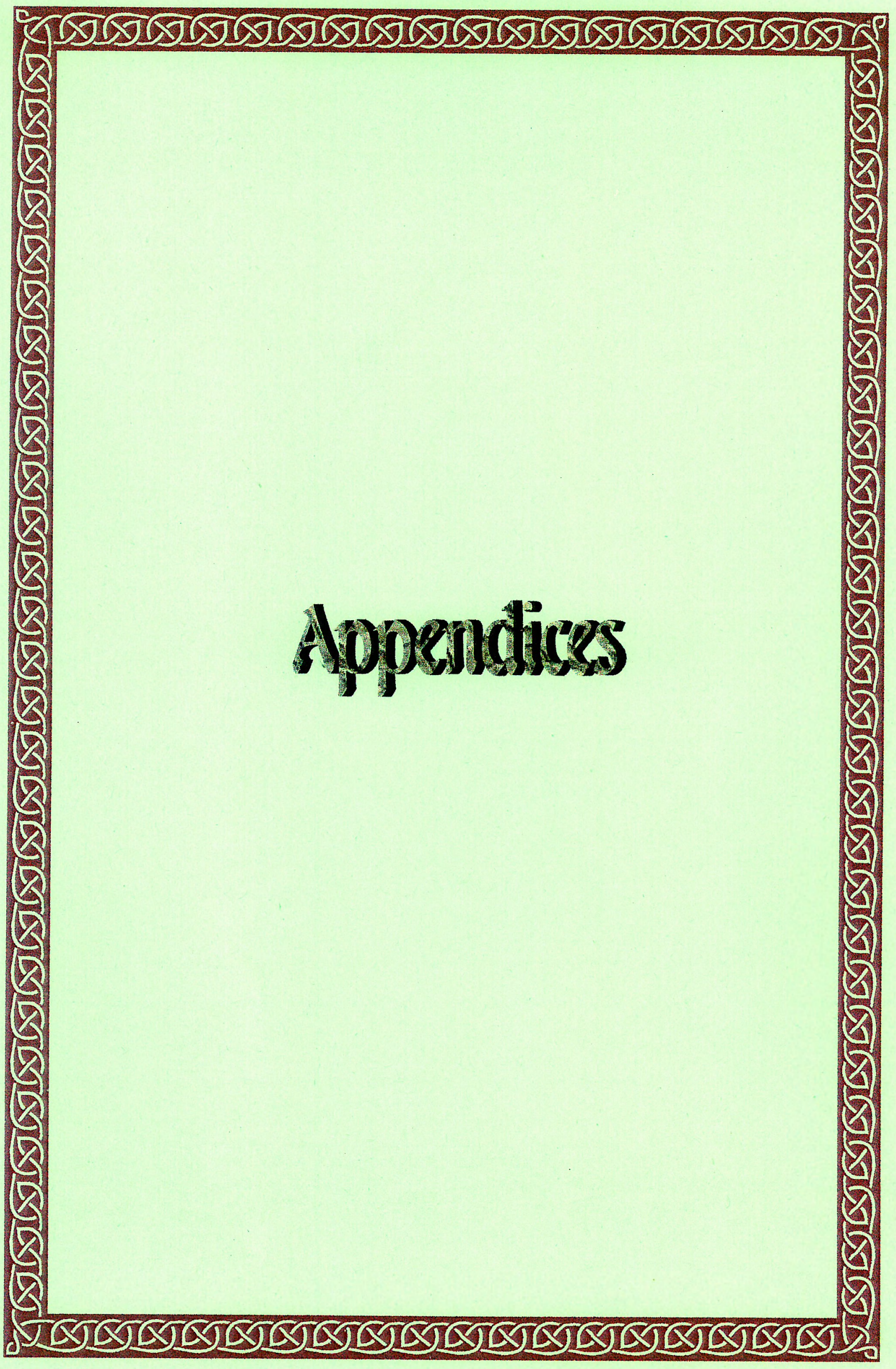




\section{Appendices}

\section{Chapter one}

1.1 Papers already published from this research

1.2 National / international platform presentations to date

\section{Chapter three}

3.1 Pilot G.P. questionnaire

3.2 Evaluation form for pilot questionnaire

3.3 Final G.P. questionnaire

3.4 Summary of quantitative data

3.5 Example of inaccurate leaflet advising bed rest

3.6 Diagnostic guide

\section{Chapter five}

5.1 Leaflet development stage 1: client questionnaire

5.2 Leaflet development stage 2: interview schedule

5.3 Final copy of the 'Back Home' leaflet

5.4 Evaluation sheet 'Tell us what you think'

5.5 G.P. consent form

5.6 Pack for recruiting clients into the study

5.7 Laminated A5 card of inclusion criteria

5.8 Client consent form

5.9 G.P. standardized empowering statement

5.10 Knowledge instrument

5.11 Attitude instruments

5.12 Satisfaction instrument

5.13 Observable behaviour instrument

\section{Chapter six}

6.1-4 Correspondence with Professor Wallston: First version of the M.H.L.C. form C 6.5 Final version of the M.H.L.C. form C

\section{Chapter eight}

8.1 Abstract of 'Back Home' study - dissemination for G.Ps

8.2 G.P. follow-up questionnaire 


\section{Appendix 1.1: Papers already published from this research}

\section{Papers / chapters}

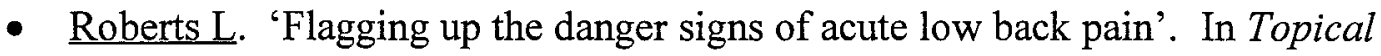
Issues in Pain II. (ed. Gifford L.) 1999 (in press).

- Little P.S., Cantrell E.C., Smith ${ }^{1}$ L., Chapman J., Langridge J., Pickering R. 'Why do GPs perform investigations?: the medical and social agendas in arranging back x-rays?' Family Practice November 1998;15(3):264-5.

- Chapman J., Smith L., Little P.S., Cantrell T., Langridge J., Pickering R. 'The "Back Home" leaflet: developing a self-management leaflet for people with acute low back pain'. Journal of Back and Musculoskeletal Rehabilitation 1997;9:61-3.

- Little P.S., Smith L., Cantrell T., Chapman J., Langridge J., Pickering R. 'General Practitioners' Management of Acute Back Pain: a Survey of Reported Practice Compared with Clinical Guidelines'. British Medical Journal 1996;312:485-8.

- Smith L., Chapman J., Cantrell T., Little P., Langridge J., Pickering R. "The 'Back Home' Study: a Working Paper. Southampton Health Journal 1996;12 (3):8-11.

\section{Conference proceedings}

- Roberts L.C., Little P., Chapman J.A., Cantrell E.G., Langridge J.C., Pickering R.M. 'Danger signs in lumbar spine assessment'. Proceedings of the 13th International Congress of the World Confederation for Physical Therapy. May 24-28 1999, Yokohama, Japan;219.

\footnotetext{
${ }^{1}$ Roberts (née Smith)
} 
- Smith L.C., Chapman J., Cantrell E.C., Little P., Pickering R., Langridge J. 'The "Back Home" Study: leaflets can change patients' knowledge and behaviour!' Proceedings of the Society for Back Pain Research. September 1997, Middlesbrough.

- Cantrell E.C., Smith L.C., Little P., Chapman J., Langridge J. 'Perception differences (GPs and radiologists): the value of plain x-ray for acute back pain'. Proceedings of the Society for Back Pain Research. October 1995, Bournemouth.

- Smith L.C., Chapman J., Cantrell E.C., Little P., Langridge J., Pickering R. 'Getting the Measure of Acute Low Back Pain'. Proceedings of the 12th International Congress of the World Confederation for Physical Therapy. June 25-30 1995, Washington DC, USA; 940.

\section{Other}

- Smith L.C., Chapman J., Cantrell E.C., Little P., Langridge J., Pickering R. 'Getting the Measure of Acute Low Back Pain' (abstract). Physiotherapy 1997;83(7):365.

- Smith L., Chapman J., Cantrell T., Little P., Pickering R., Langridge J. The "Back Home" Study: The practical value of a diagnostic protocol and patient information leaflet in the home management of back pain. Research and Development project grant R52.93 (research report). 


\section{Appendix 1.2: National / international platform presentations}

(given by the researcher on work in this thesis to date)

\begin{tabular}{|c|l||}
\hline Date & \multicolumn{1}{|c|}{ Details of conference } \\
\hline May '99 & $\begin{array}{l}13^{\text {th }} \text { World Confederation for Physical Therapy Congress, Yokohama, Japan } \\
\text { Platform presentation: 'Danger signs in lumbar spine assessment' }\end{array}$ \\
\hline Sept '97 & $\begin{array}{l}\text { Chartered Society of Physiotherapy Congress, Edinburgh. } \\
\text { Platform presentation: 'Getting the Measure of Acute Low Back Pain'. }\end{array}$ \\
\hline Sept '97 & $\begin{array}{l}\text { Society for Back Pain Research, Middlesbrough. } \\
\text { Poster and presentation: 'The "Back Home" study: leaflets can change patients } \\
\text { knowledge and behaviour'. }\end{array}$ \\
\hline Jun '97 & $\begin{array}{l}\text { The Physiotherapy Pain Association, Bristol. } \\
\text { Lecture: 'Flagging up the danger signs of acute low back pain'. (For report see } \\
\text { "Frontline' July 2, 1997, page 18). }\end{array}$ \\
\hline Jun '95 & $\begin{array}{l}12^{\text {th }} \text { World Confederation for Physical Therapy Congress, Washington DC, USA } \\
\text { Platform presentation: 'Getting the Measure of Acute Low Back Pain'. }\end{array}$ \\
\hline
\end{tabular}




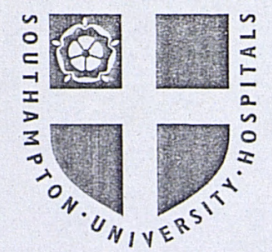

3.1 Pilot questionnaire

Dear Doctor, RE: CURRENT PRACTICE IN THE MANAGEMENT
OF LOW BACK PAIN

Please complete the Questionnaire by reading the statements and placing a tick in the box that most appropriately reflects your response.

Please answer the questions honestly! We are not interested in 'perfect practice', but we would like to know what current practice really is, (and this questionnaire will not include your name)!

1.1 In approximately what \% of acute low back pain patients do you advocate bed rest?

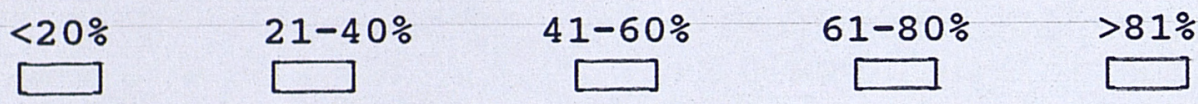

1.2 Do you specify a duration for resting in bed?

NO []$]$ If yes, please specify ............... days.

2. Do you give any specific advice regarding the following? Please tick the appropriate box and give a brief description of your advice.
a) Sleeping $\backslash$ mattress
b) Changing positions
c) Driving
d) Exercises
e) Sitting
f) Lifting
g) Gardening

NO YES

3. Do you:-

NO YES
a) Automatically follow the patient up?
b) Wait for the patient to seek further appointments with you?
c) Wait for the patient to seek telephone advice from you?


4.1 What criteria do you use when deciding whether $\mathrm{x}$-rays are appropriate for a patient with acute low back pain?

4.2 Approximately what \% of acute low back pain patients do you send for $x$-ray?

$$
\text { .................... }
$$

NO $\underline{\text { YES }}$

5. Do you give out any back pain leaflets?

IF YES, PLEASE ENCLOSE A COPY.

6.1 Do you routinely sign a sickness certificate for a first episode of acute low back pain?

6.2 If yes, what is the usual length of sickness certification that you sign:-

7 days 10 days 14 days 21 days 28 days other

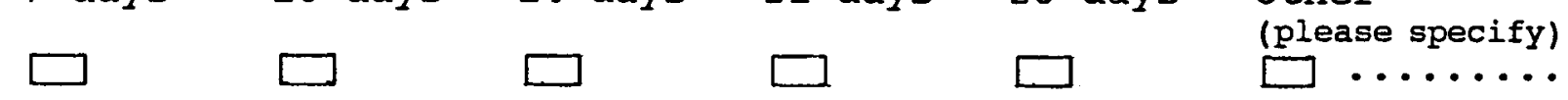

7. If people ask how long it will take to recover, what do you say?

8. How many patients do you see per month with AcUTE low back pain?

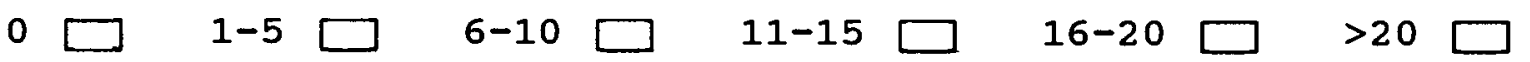

9.1 What signs and symptoms do you consider are 'danger signs' for an acute back pain patient, i.e. those which would necessitate (immediate) hospital attention?

Please specify

9.2 Do you routinely test the following in a first examination of an acute low back pain patient?

Straight leg raise $\frac{\text { NO }}{\square} \frac{\text { YES }}{\square}$

Reflexes $\square \square$ If yes, please specify which one(s) 
Muscle weakness $\quad \frac{\text { NO }}{\square} \frac{\text { YES }}{\square}$ If yes, please specify which muscles

Sensation

$\square \quad \square$ If yes, please specify modality (i.e pin prick,light touch etc

Palpation

10. Approximately what $\%$ of your patients with acute low back pain are eventually referred to different practitioners please place percentage numbers in the box to the right of the statement.

$\%$

- Hospital Consultant - Orthopaedic surgeon...

- Rheumatologist ...... $\square$

- Neurologistl

Neurosurgeon

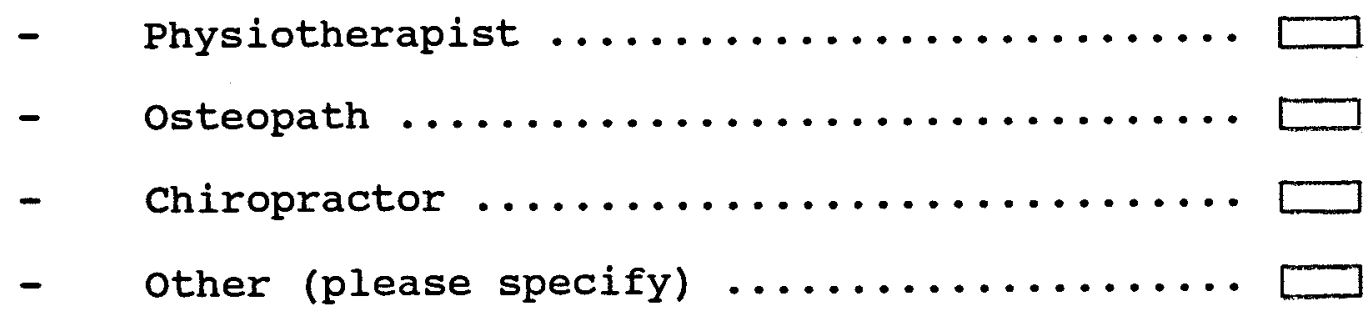

11. In approximately what $\%$ of acute low back pain episodes would you advocate the following drugs - please place percentage numbers in the box to the right of the statements.

$\%$

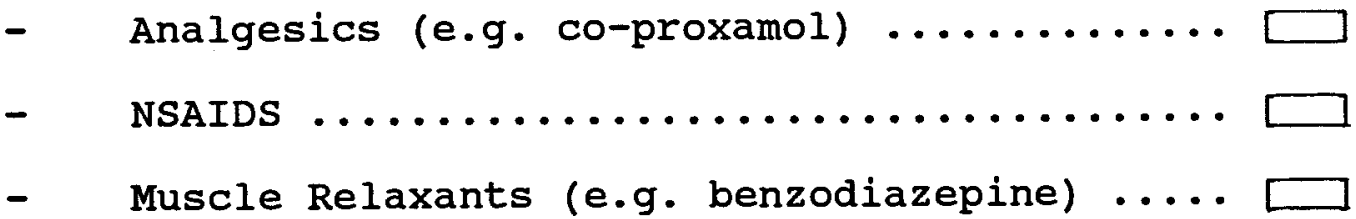

12.1 How many years have you been practising as a GP? ............ Years

12.2 What year did you enter General Practice? ..........

13. Have you completed any post-graduate courses on the management of acute low back pain?

No [ ]

YES [] If yes, please specify course .............. duration ................ (days)

which year 
14. Please mark with a vertical line on the scale below to indicate how satisfied you feel with your current approach to the management of acute low back pain:

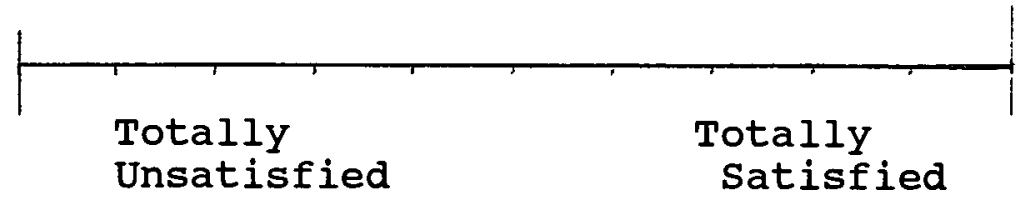

15. Please include any additional comments regarding your current management approach here.

THANK YOU FOR YOUR COOPERATION:

please return this form in the enclosed stamped addressed envelope to:

Lisa Smith
Research Physiotherapist
Physiotherapy Department
Southampton University Hospitals Trust
Tremona Road
Southampton SO9 $4 \mathrm{XY}$
Telephone : 0703796458

Ted Cantrell - Consultant Rheumatologist

Judith Chapman - Lecturer, School of Occupational \& Physiotherapy

John Langridge - Manager of Physiotherapy Services

Paul Little - Research Fellow in Academic General Practice 


\section{TELL US WHAT YOU THINK}

After completing the questionnaire "Current Practice in the Management of Acute Back Pain", please tell us what you think. Please complete this evaluation sheet by placing a tick in the box which reflects your opinion.

I found the questions:

$\begin{array}{lll}\text { Strongly Agree } & \text { Disagree } & \begin{array}{l}\text { Strongly Conments } \\ \text { Disagree }\end{array}\end{array}$

1. Easy to follow

2. Relevant to my current practice

3. Well constructed

4. Too time consuming

5. Easy to complete

6. Too detailed

7. Covered all aspects of my current management of acute low back pain

YES [ ] No [ ] - Please specify any omissions:-

Length of time taken to complete GPs questionnaire Any additional comments

Thank you for your cooperation.

Please return this evaluation sheet together with the completed GP questionnaire to:

Lisa Smith

Research Physiotherapist Physiotherapy Department Southampton University Hospitals Tremona Road Southampton SO9 $4 \mathrm{XY}$ 
BACK PAIN RESEARCH PROJECT

QUESTIONNAIRE

\section{CURRENT PRACTICE IN THE MANAGEMENT OF LOW BACK PAIN}

\section{Instructions:}

Please complete the Questionnaire by reading the statements and placing a tick in the box that most appropriately reflects your response.

Please answer the questions honestly! We are not interested in 'perfect practice', but we would like to know what current practice really is. Your responses to this questionnaire are kept confidential.

1. Do you give out any leaflets on back pain?

\section{IF "YES", PLEASE ENCLOSE A COPY}

2. Do you routinely test the following in a first examination of a patient with acute low back pain?

$$
\text { YES NO }
$$

Straight leg raise

Reflexes

if yes, please specify which one(s).

Muscle weakness

if yes, please specify which muscles.

Sensation

$\square \quad \square$ if yes, please specify modality

(i.e pin prick, light touch etc).

Palpation of spine 
3. What signs and symptoms do you feel justify immediate hospital advice, for a patient with acute low back pain?

\section{YES NO}
a) Severe local back pain
b) Bilateral leg signs
c) Loss of reflex at one level
d) Extensor plantar response
e) Constant night pain
f) Unilateral sciatic symptoms below the knee
g) Saddle anaesthesia
h) Neurological signs at multiple levels

4. Do you give any specific advice regarding the following? Please give a brief description of your advice.

\section{YES NO}
a) Sleeping/mattress
b) Driving
c) Specific back exercises
d) General fitness
e) Sitting
f) Lifting
g) Gardening

5. Do you routinely sign a sickness certificate for a person with a first episode of acute low back pain?

\section{YES NO}
a) Back pain alone
b) Root signs 
6. What criteria do you use when deciding whether $\mathrm{X}$-rays are appropriate for a patient with acute low back pain?

Medical:

Social:

7. Approximately what $\%$ of patients with acute low back pain do you send for X-ray? $\%$

8. What $\%$ of your patients with acute low back pain do you (yourself) treat with:

$\begin{array}{ccc}- & \text { Acupuncture } & \\ - & \text { Manipulation }\end{array}$

9. Do you encourage your patients with back pain alone to take responsibility for making follow-up appointments with you?
Always
Usually
Sometimes
Never

10. How many years have you been practising as a GP? Years.

11. What is your consultation interval in routine surgeries? Minutes.

12. Have you completed any post-graduate courses specially about the management of acute low back pain?

NO

YES $\square$ If yes, please specify:

Course:

Duration of Course: 
13. Please mark with a vertical line on the scale below, to indicate how satisfied you feel with your current approach to the management of acute low back pain:

\begin{tabular}{ll} 
Lotally & \\
Unsatisfied & Totally \\
\hline & Satisfied
\end{tabular}

14. Please include any additional comments regarding your current management approach, of patients with acute low back pain.

\section{THANK YOU FOR YOUR CO-OPERATION}

Please return this form in the enclosed stamped addressed envelope to:

\section{Lisa Smith}

Research Physiotherapist

Physiotherapy Department

Southampton University Hospitals Trust

Tremona Road

Southampton

S09 4XY

Telephone: 0703796459

\section{RESEARCH TEAM:}

Ted Cantrell - $\quad$ Consultant Rheumatologist

Judith Chapman - $\quad$ Lecturer, School of Occupational Therapy and Physiotherapy

John Langridge - $\quad$ Manager of Physiotherapy Services

Paul Little - - $\quad$ GP and Research Fellow in Academic General Practice

Lisa Smith $\quad$ - $\quad$ Research Physiotherapist 
ID. No:

3.4 Data summary

\section{BACK PAIN RESEARCH PROJECT}

QUESTIONNAIRE

CURRENT PRACTICE IN THE MANAGEMENT OF LOW BACK PAIN

\section{Instructions:}

Please complete the Questionnaire by reading the statements and placing a tick in the box that most appropriately reflects your response.

Please answer the questions honestly! We are not interested in 'perfect practice', but we would like to know what current practice really is. Your responses to this questionnaire are kept confidential.

$\frac{\text { SUMMARY OF } 1166 \text { RESULIS }}{\text { (QUANTITATIVE) }}$

1. Do you give out any leaflets on back pain?

2. Do you routinely test the following in a first examination of a patient with acute low back pain?

$$
\text { YES NO }
$$

Missing

Straight leg raise $\quad \square 147 \quad 16 \square \quad 03$

Reflexes

$\square 117 \quad 44 \square$ if yes, please specify which one(s).

$\infty$

Muscle weakness

$\square 64$

$92 \square$

if yes, please specify which muscles.

10

Sensation

$\square 70$

$88 \square$

if yes, please specify modality

(i.e pin prick, light touch etc). 
3. What signs and symptoms do you feel justify immediate hospital advice, for a patient with acute low back pain?

YES NO Missing
a) Severe local back pain
$\square 12$
144
10
b) Bilateral leg signs
$\square 84$
$\square 73$
$\infty$
c) Loss of reflex at one level
$\square 18$
$\square 136$
12
d) Extensor plantar response
$\square 83$
$\square 67$
16
e) Constant night pain
$\square 47$
$\square 103$
16
f) Unilateral sciatic symptoms below the knee
$\square$ C4
$\square 148$
14
g) Saddle anaesthesia
$\square 147$
$\square 10$
09
h) Neurological signs at multiple levels
$\square 135$
$\square 24$
07

4. Do you give any specific advice regarding the following?

Please give a brief description of your advice.

YES NO MISSING
a) Sleeping/mattress
$\square 148$
$\square 15$
03
b) Driving
$\square 108$
$\square 56$
02
c) Specific back exercises
$\square 95$
$\square 68$
03
d) General fitness
$\square 105$
$\square 53$
08.
e) Sitting
$\square 134$
$\square 28$
94
f) Lifting
$\square 159$
$\square 05$
02
g) Gardening
$\square 82$
$\square 76$
08

5. Do you routinely sign a sickness certificate for a person with a first episode of acute low back pain?

YES NO MSSING
a) Back pain alone
$\square 61$
$\square 97$
08
b) Root signs
$\square, 120$
口38
08 
6. What criteria do you use when deciding whether $\mathbf{X}$-rays are appropriate for a patient with acute low back pain?

Medical:

Social:

7. Approximately what $\%$ of patients with acute low back pain do you send for X-ray? $\underline{\%}$

8. What \% of your patients with acute low back pain do you (yourself) treat with:

$\begin{array}{ll}- & \text { Acupuncture } \\ - & \text { Manipulation }\end{array}$

9. Do you encourage your patients with back pain alone to take responsibility for making follow-up appointments with you?

$\begin{array}{lllll}\text { Always } & \text { Usually } & \text { Sometimes } & \text { Never } & \text { Mossine } \\ \square 44 & \square 97 & \square 21 & \square 02 & 02\end{array}$

10. How many years have you been practising as a GP? Years.

11. What is your consultation interval in routine surgeries? Minutes.

12. Have you completed any post-graduate courses specially about the management of acute low back pain?

NO

YES $\square$ If yes, please specify:

Course:

Duration of Course: 
13. Please mark with a vertical line on the scale below, to indicate how satisfied you feel with your current approach to the management of acute low back pain:

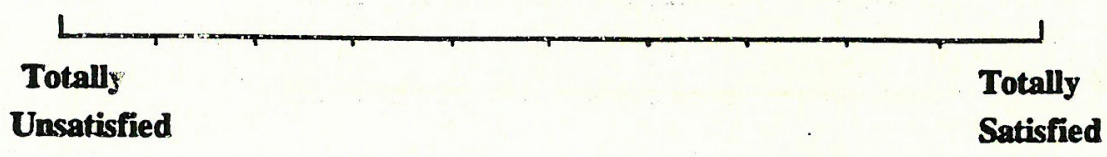

14. Please include any additional comments regarding your current management approach, of patients with acute low back pain.

\section{THANK YOU FOR YOUR CO-OPERATION}

Please return this form in the enclosed stamped addressed envelope to:

\section{Lisa Smith}

Research Physiotherapist

Physiotherapy Department

Southampton University Hospitals Trust

Tremona Road

Southampton

S09 4XY

Telephone: 0703796459

\section{RESEARCH TEAM:}

Ted Cantrell - Consultant Rheumatologist

Judith Chapman - Lecturer, School of Occupational Therapy and Physiotherapy

John Langridge - $\quad$ Manager of Physiotherapy Services

Paul Little - $\quad$ GP and Research Fellow in Academic General Practice

Lisa Smith - Research Physiotherapist 


\section{BED REST FOR BACK PAIN}

Bed rest means spending 7 - 14 days horizontally on a firm bed with your knees bent (see diagram). Unfortunately sitting in a chair will put pressure on your spine which will not help your back.

Bed rest resolves $90 \%$ of back problems within three weeks. However, it is important that you follow your GP's instructions carefully.

Pain killers can be taken to help the pain (please consult your doctor for advice).

If you can feel improvement after $7-14$ days, bed rest should be continued for up to 6 weeks.

If you feel no improvement after two weeks, and you have been following this treatment correctly, you should tell your GP.
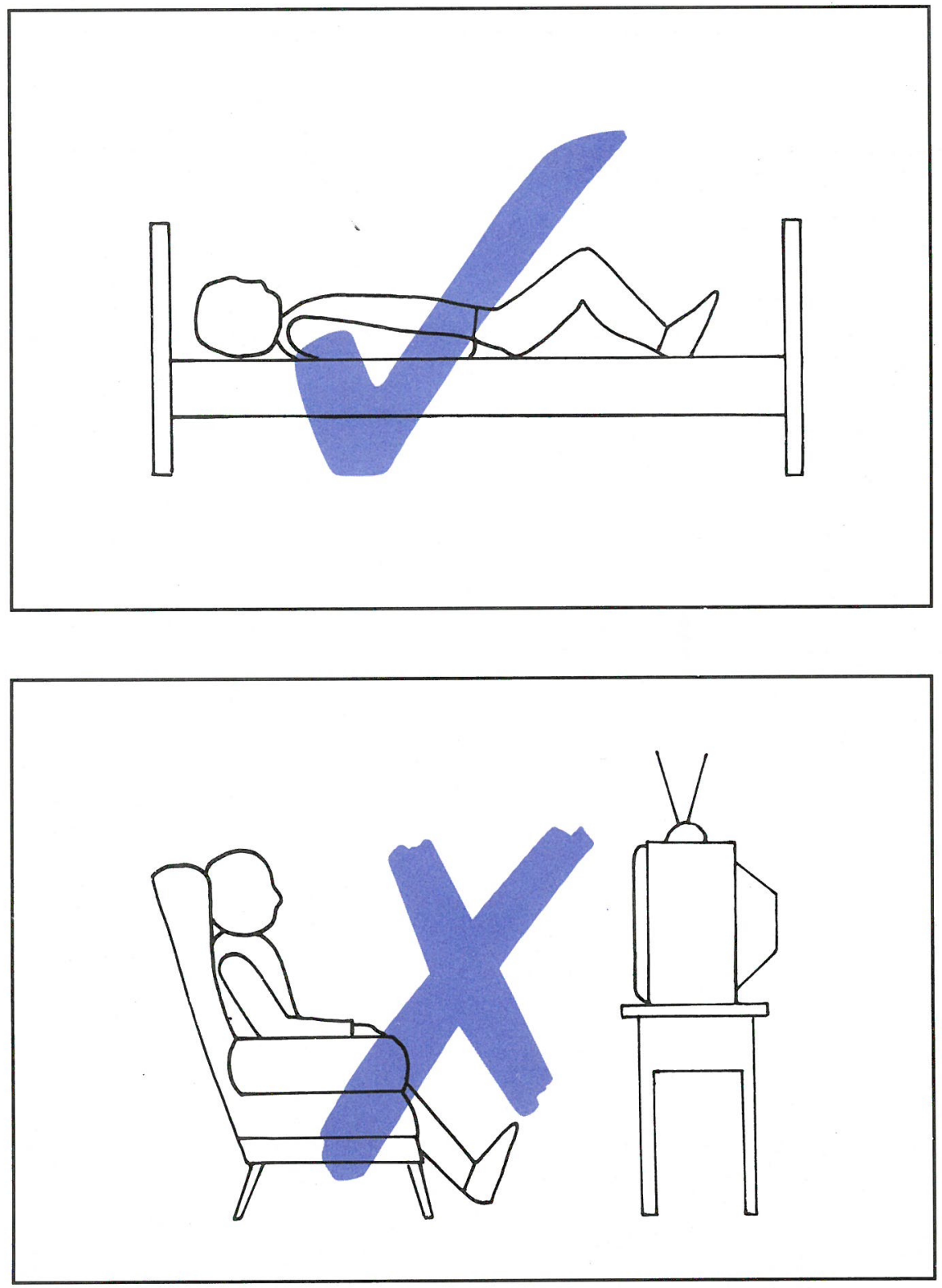

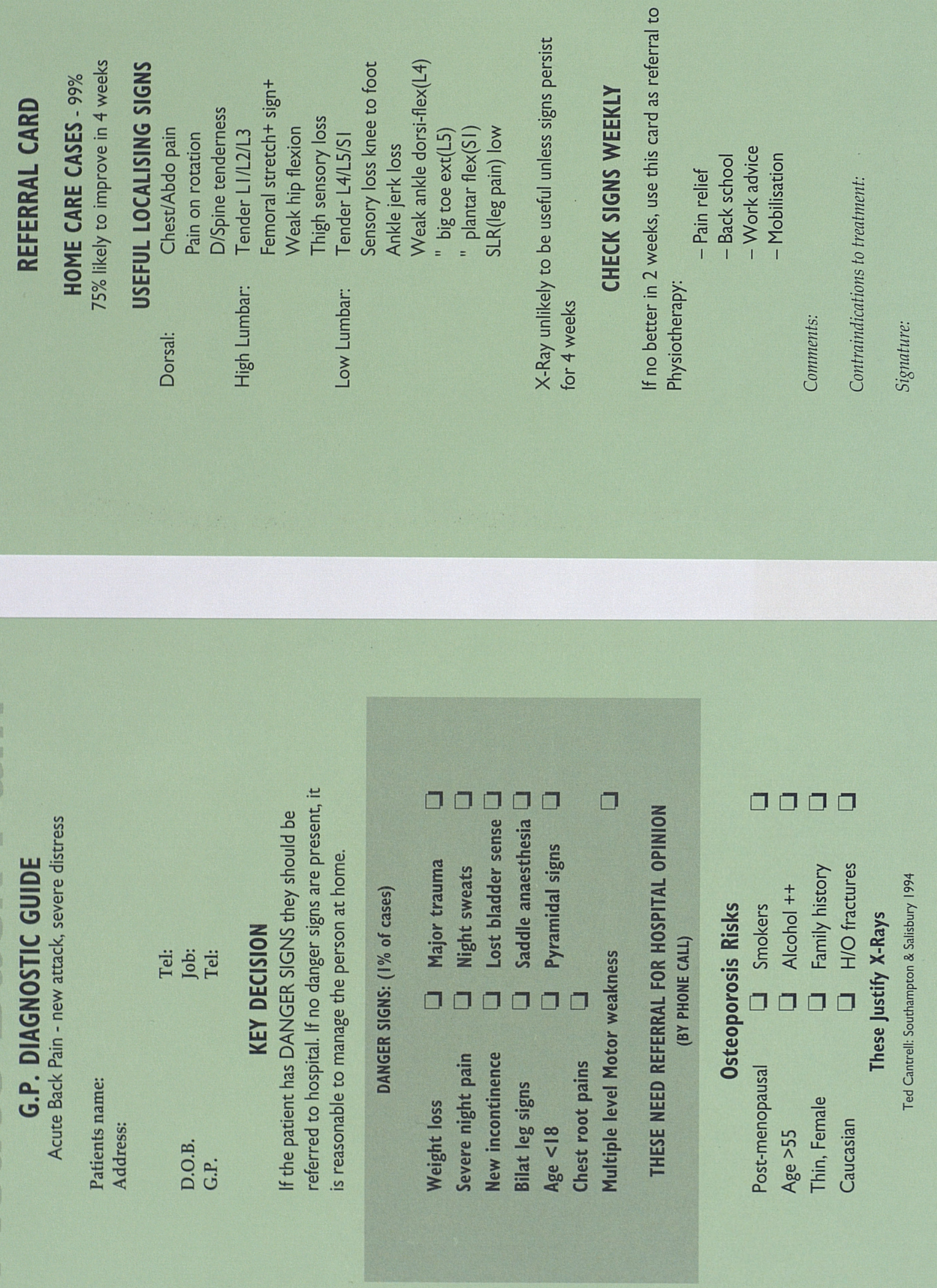
From: Dr. E Cantrell, Rheumatologist, Southampton General Hospital

Ms. J Chapman, Lecturer, School of Physiotherapy, University of Southampton

Mr. J Langridge, Director Physiotherapy Services, Southanupton University Hospitals Unit

Dr. P Little, Research Fellow in Academic General Practice, University of Southampton

Ms. L Smith, Physiotherapist \& Research Assistant

We are preparing a leaflet on how to manage acute low back pain.

Please could you help us?!

We would like to know what information you would find helpful in managing your low back pain.

Please answer the question on this page first. Then turn over and complete the questionnaire on the back. Please place your answers in this stamped, addressed envelope.

You do not need to put your name on this paper, so your answers will be quite confidential.

1. Please use the rest of the space on this page to list any questions that you would like to ask your doctor about low back pain and how to manage it. (List any questions that you have already asked your doctor too) 
2. How important are the following items for you to know about now? For each item, place a tick in the column that best applies to you.

1. How to position myself (Lying, sitting, standing)

2. How to sleep or rest comfortably

3. How to get about

4 How to do my daily tasks

5. The easiest way to get washed and dressed

6. Exercises

7. How to lift things

8. Getting back to work

9. How to drive

10. How to control my pain by using tablets

11. How to control my pain by using self-help measures (eg. liniments, hot water bottles)

12. Where to get help

13. Getting back to sports

14. How to cough and sneeze

15. Getting back to DIY

16. Getting back to gardening

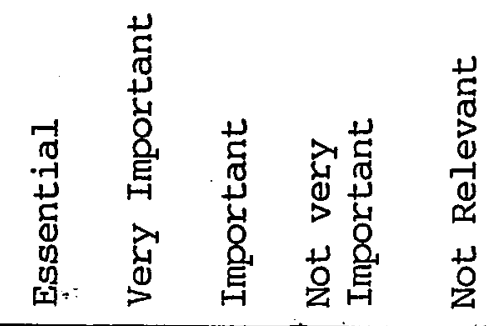

\begin{tabular}{|c|l|l|l|l|}
\hline 13 & & & & \\
\hline 14 & & & & \\
\hline 5 & & & & \\
\hline 12 & & & & \\
\hline 7 & & & & \\
\hline 13 & & & & \\
\hline 16 & & & & \\
\hline 13 & & & & \\
\hline 11 & & & & \\
\hline 6 & & & & \\
\hline 10 & & & & \\
\hline 10 & & & & \\
\hline 7 & & & & \\
\hline 6 & & & & \\
\hline 4 & & & & \\
\hline 6 & & & & \\
\hline
\end{tabular}


1) What bothers you most about your back pain?

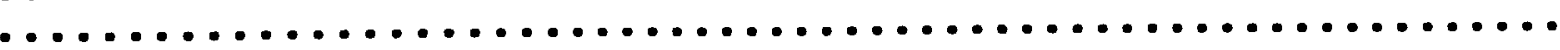

2) Is there anything you find difficult at the moment?

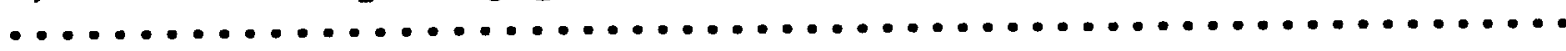

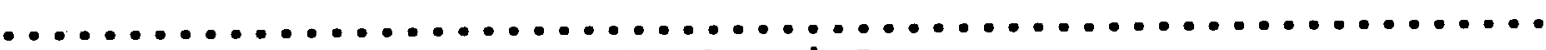

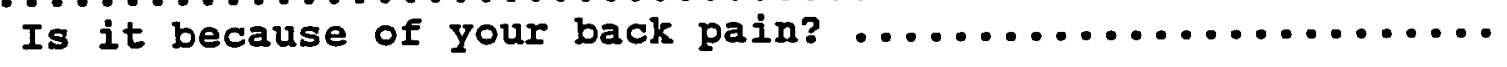

3) Is it your first episode of back pain?

4) Do you find that your back pain is worse at any particular time of day?

5) Does any particular activity make your back pain worse?

6) How do you balance the pain with the gain? ie. if you know that something is likely to hurt your back, do you not do it or bear the pain?

7) Do you ask for help or do you struggle?

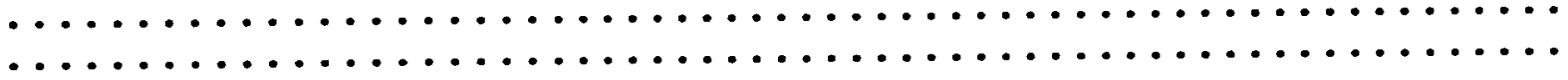

8) If you stir your back pain up doing something, what do you do?

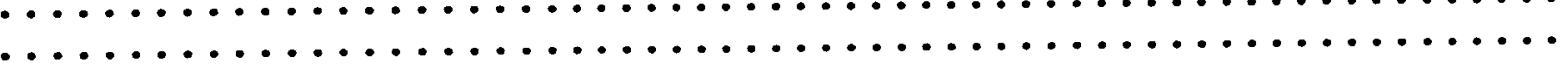

9) If you stir your pain up, how quickly does it settle?

10) What would you most like advice about? It may be from your doctor, physio or other health professional).

11) If a leaflet was written for someone with back pain, what

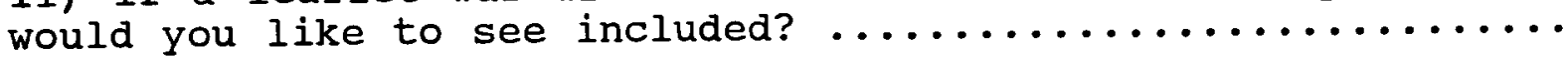

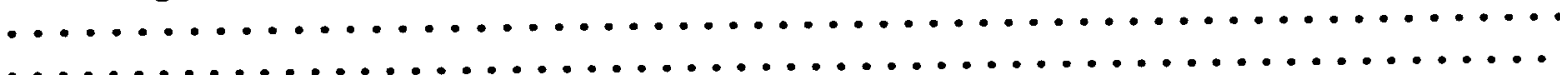




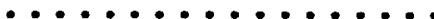

$\ldots \ldots$

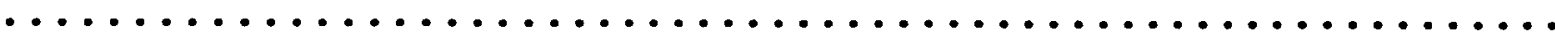

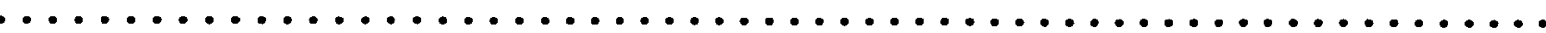

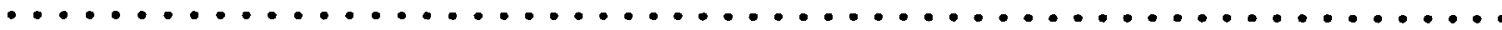

12) How important do you think it is to include the following? (See sheet).

13) Which five of these do you think are the most important? 1st.

2nd.

$3 r d$

4 th

5 th

14) This leaflet is being developed at the moment for people with low back pain and it is hoped a person will be given it by their doctor when they first go and see him/her. What do you think of it?.

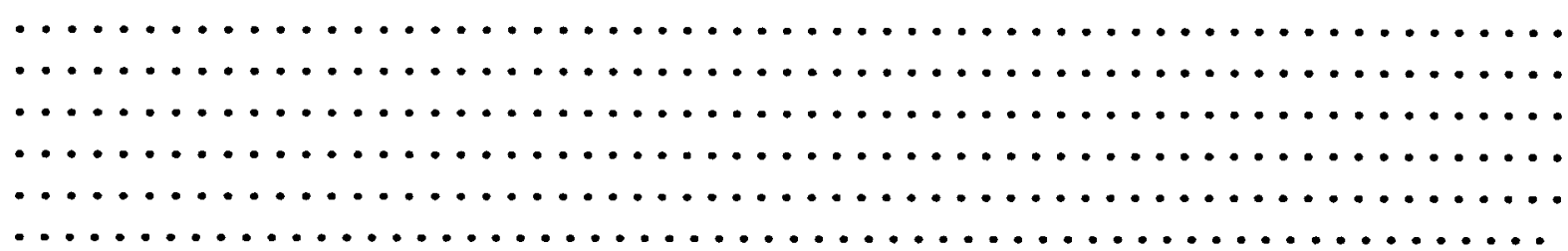

15) Is there anything missing that you think would be important?

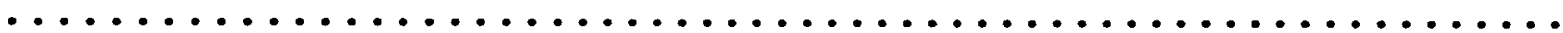

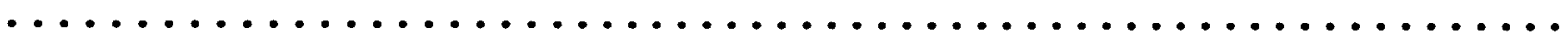

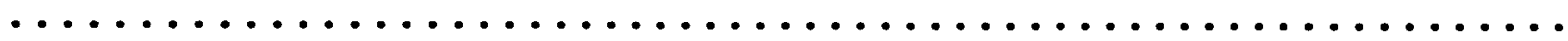

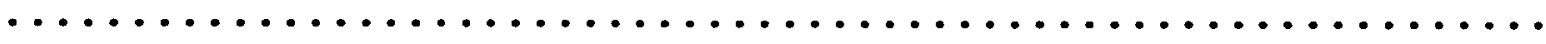

16) Is the information relevant? Could anything be taken out?

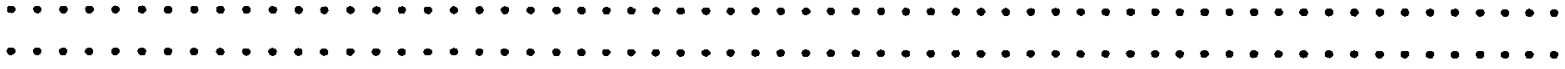

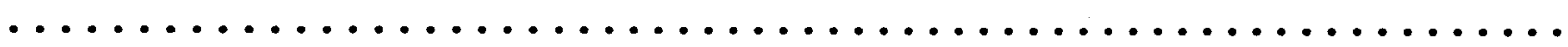

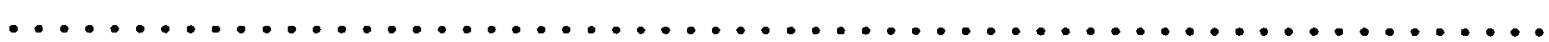

17) How useful would something like this have been to you when you first went to see your doctor?

18) Any other comments?

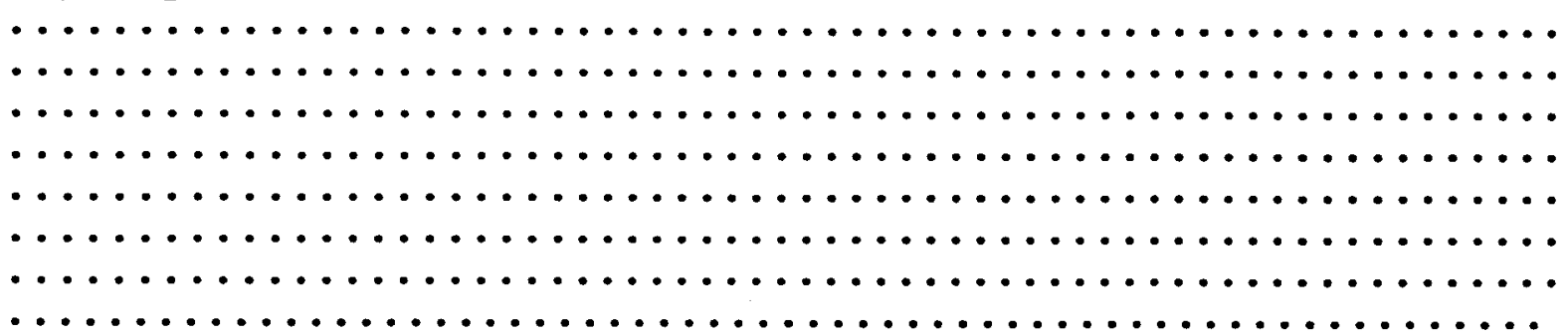

Thank you for your time and effort! 
How to

- position myself (lying, sitting etc)

- sleep or rest comfortably

- get about

- do my daily tasks

- get washed or dressed easily

- lift things

- drive

- control my pain using tablets

- control my pain using self-help measures (eg. hot water bottle, ice packs etc)

- cough or sneeze

- modify my life to prevent my back pain coming back

- manage the shopping

- modify my bedding \& pillows to help my pain

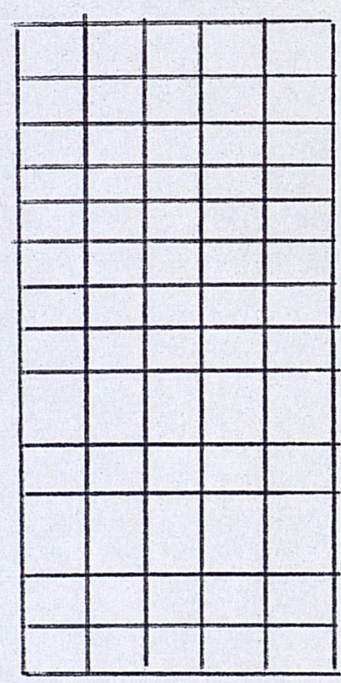

what

- exercises to do

- treatment is available

- are some of the causes of low back pain

- can I do to get/keep myself fit

- makes up the spine

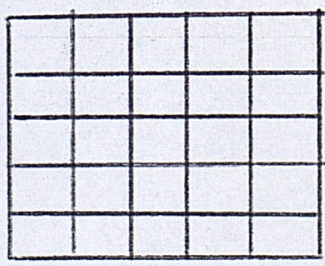

getting back- to social and leisure activities

- to work

- to sports

- to D.I.Y.

- to gardening

- to physical relationships

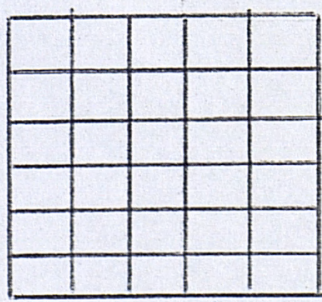

where - to get help/further advice 


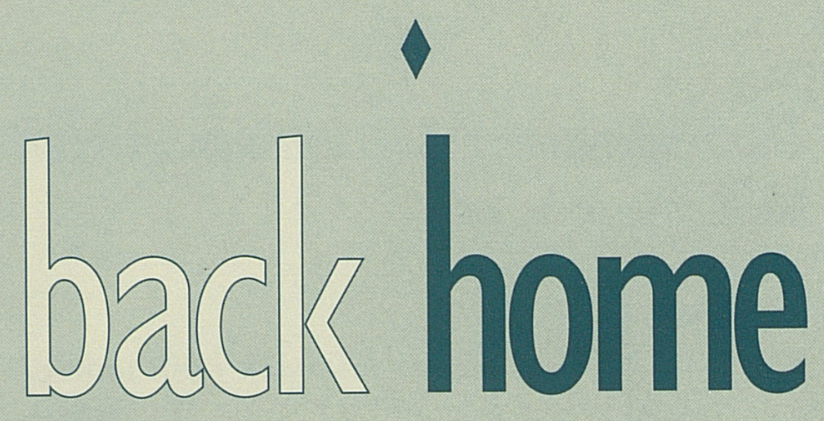

How to look after

an attack

of low back pain

at home

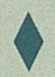

... you can ease your pain!

$\checkmark$

... most people do get better within

four weeks

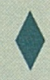


This leaflet aims to help you manage your back pain at home.

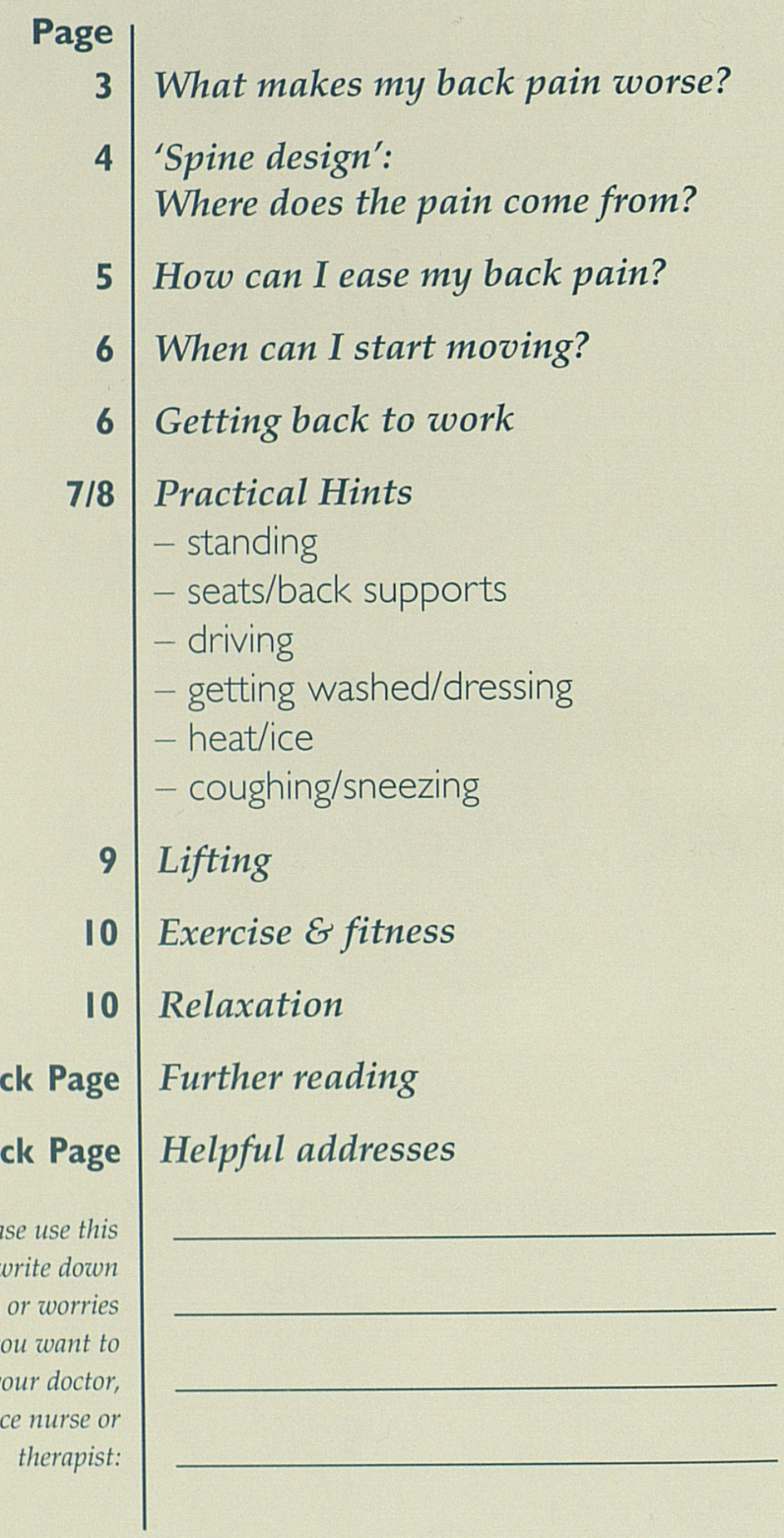

\section{What makes my back pain worse?}

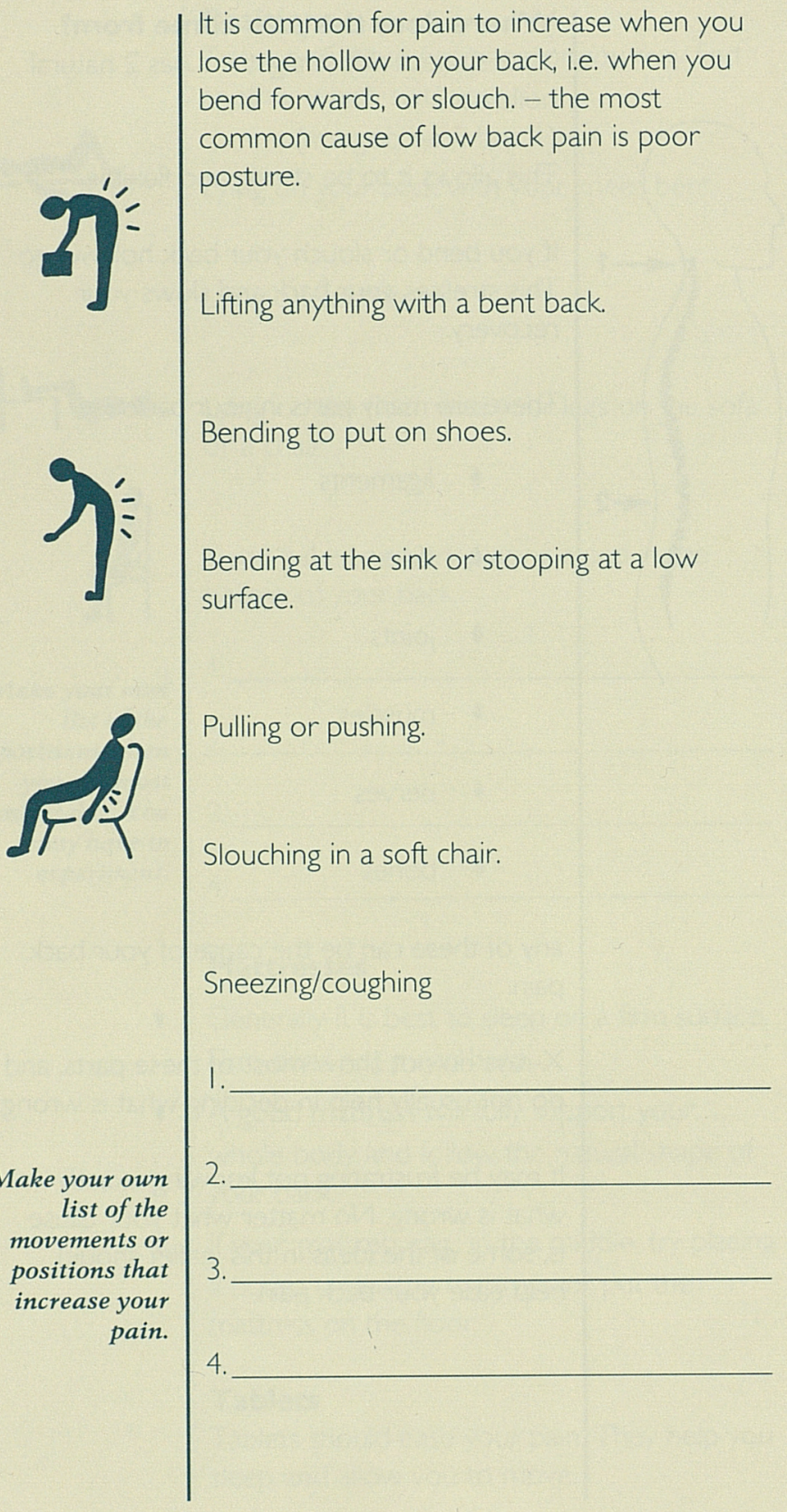

3 


\section{Spine Design}

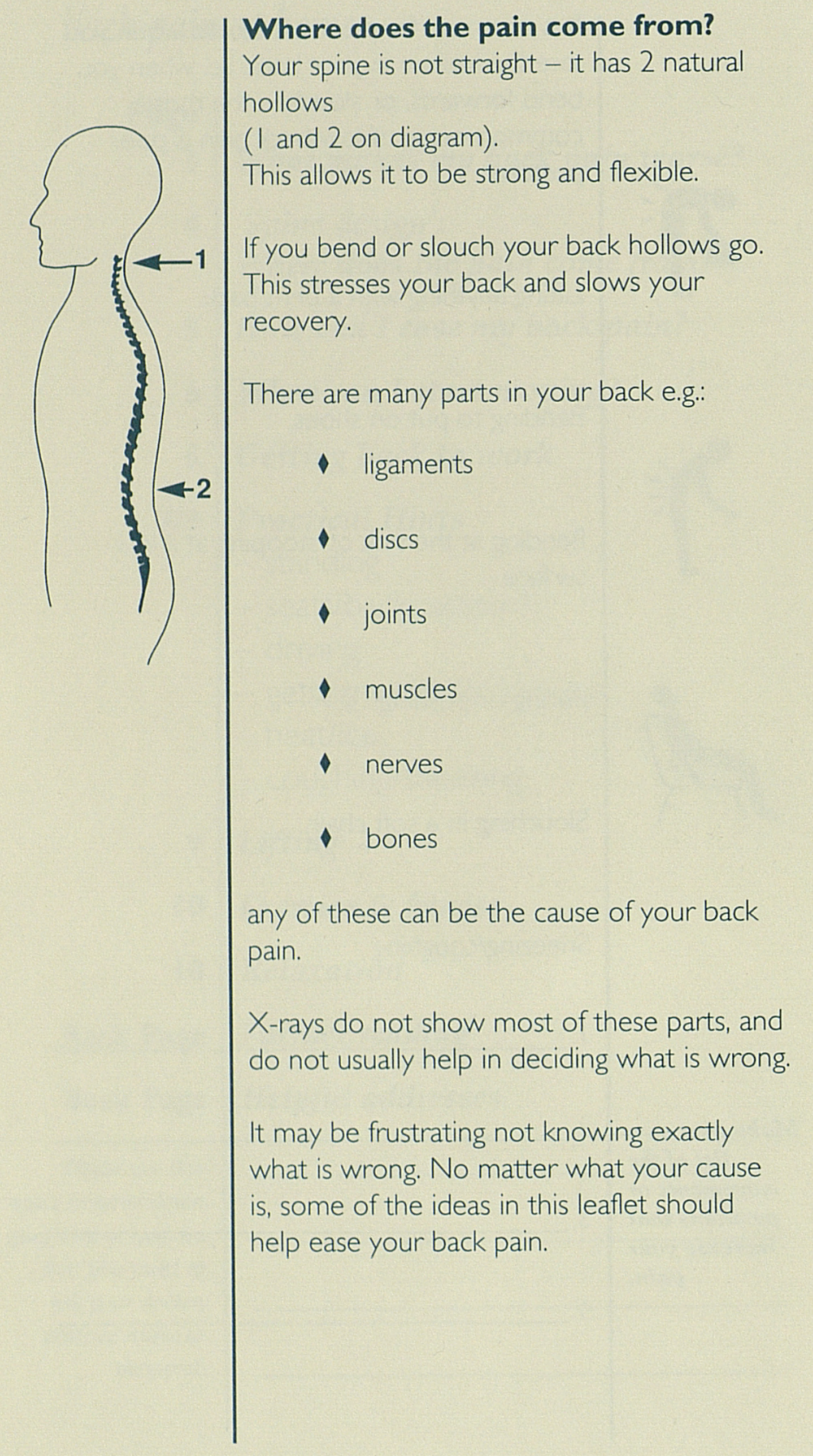

\section{How can I ease my back pain?}
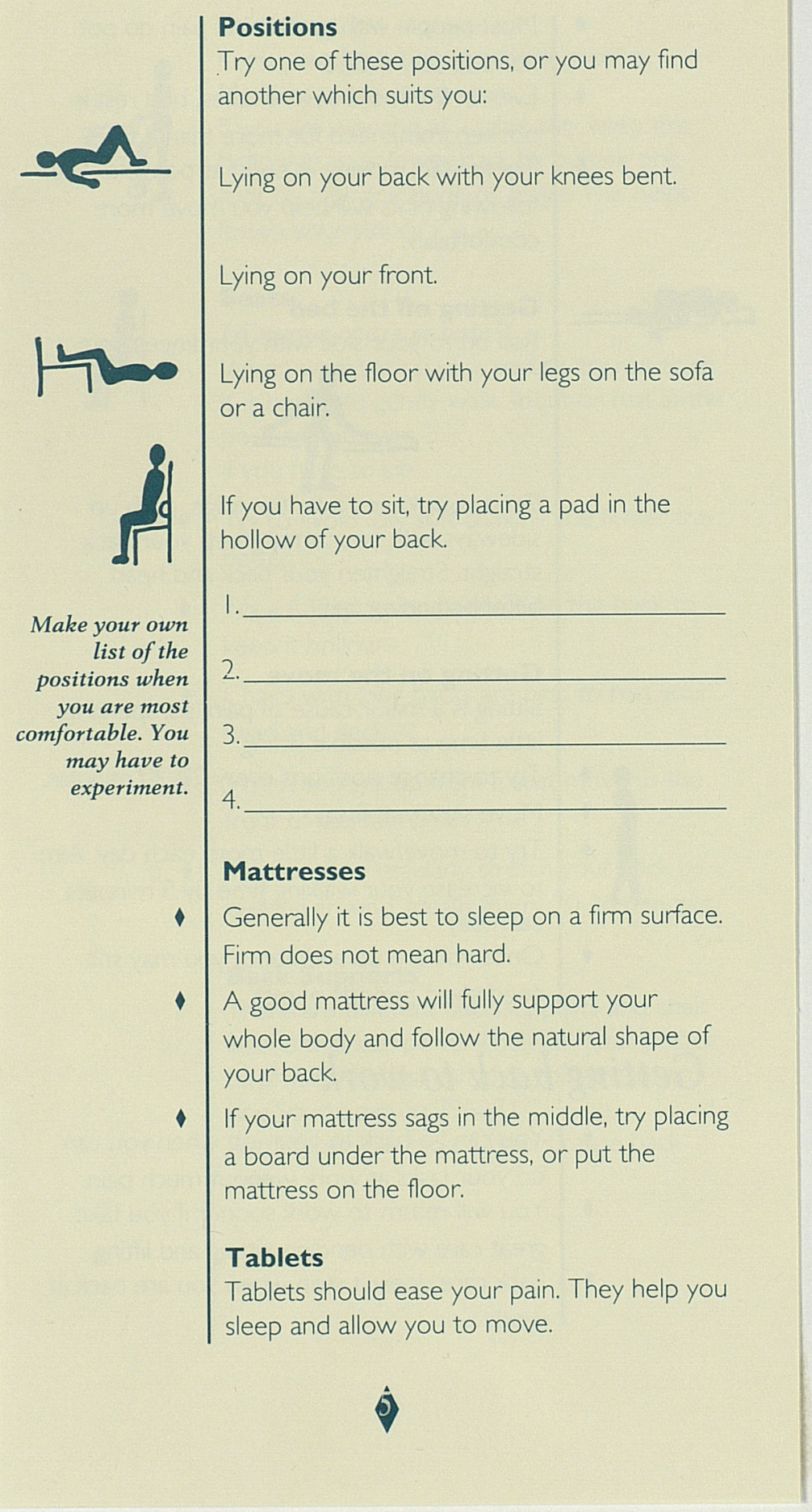


\section{When can I start moving?}

- Most people with acute back pain do not need to stay in bed.

- Even in the more severe cases, bed rest is not recommended for more than 2 days.

- Try to start moving as soon as possible. The following hints will help you move more comfortably.

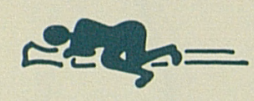

Getting off the bed

Roll on to your side with your knees bent

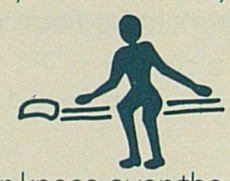

Bend your knees over the edge, push up sideways with your arms. Keep your back straight. Straighten your back and head before standing up.

\section{Getting on the move}

Sitting is a major cause of pain, so, spend as little time as possible sitting.

j:

Try to change positions every 20-30 minutes. Move slowly at first.

Try to move/walk a little more each day. Aim to increase your walking time by 5 minutes each day.

- Once you are up and about you may still need short periods of rest.

\section{Getting back to work}

- You can go back to your job when you can do your tasks at work without much pain.

- You will return to work sooner if you take great care with bending, sitting and lifting.

- Back care doesn't stop when you are back at work.

\section{Practical Hints}

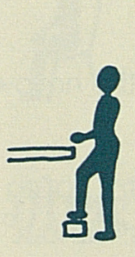

\section{Standing}

Keep your back hollow where possible.

Move as much as you can.

If you are working at a table top, keep the hollow in your lower back by putting one foot on a phone book or ledge. This helps lessen your stoop.

\section{Seats}

(A major cause of pain!)

When your back pain is severe, try not to sit. It is better to gently walk about or rest in the positions on page 5

If you have to sit: $\rightarrow$

\section{Back Supports}

There are different back supports and chair inserts that can be very helpful. Your doctor. or physiotherapist can give you advice, or ring Southampton 796631

Remember low or soft chairs cause back pain. 


\section{Practical Hints}

$\checkmark$

$\checkmark$

When your back pain is severe try not to drive for longer than 20 minutes without getting out and having a stretch.

\section{Getting Washed}

$\checkmark$

$\checkmark$

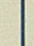
much worse because of the position you are in.

\section{Shoes}

$\checkmark$ Try putting on your socks/tights and shoes from a lying down position on the bed.

\section{Heat}

(For sore muscles)

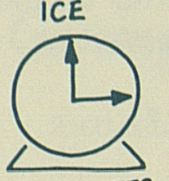

A hot water bottle wrapped in towels and placed over the painful area, or a shower, can help to ease the pain.

\section{Ice}

A bag of crushed ice or a bag of frozen peas wrapped in a towel and placed over the painful area can help to ease the pain. (If you use ice or heat use it for 15 minutes only and check that your skin is not blotchy red, which is a sign to stop).

\section{$\{\geqslant 8$}

Lifting

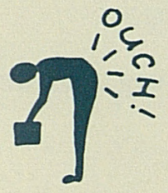

This is not just lifting at work - it may be lifting a baby, the shopping, even a sock! Be very careful when lifting people, animals etc.

Plan. Do I have to lift this now? If you do, follow this checklist:

$\checkmark$ Get as close to the object as possible.

$\checkmark$ Bend your knees, not your back.

$\checkmark$ Straighten your head and look up before lifting (1 on diagram)

$\checkmark$ Keep your arms close to your body (2 on diagram).

Follow the same rules when lowering an object. $\checkmark$ can be very helpful.

Even if you cannot lift exactly as advised, try to include as many of these points as possible. 


\section{Exercise \& Fitness}

- People in poor physical shape are likely to get back pain.

There are no universal exercises for back pain - it depends on the nature of your back problem, so seek professional advice before doing any specific exercises.

- Walking helps most recovering backs.

For any exercise, try following a basic guideline:

Always warm-up by:

- beginning slowly

progressing gradually.

- respect any pain.

- avoid collapsing in a soft chair afterwards! (remember the advice on Page 7)

Some of the easier sports to get back to after having back pain include walking, swimming, yoga, aquarobics, Tai Chi, etc.

\section{Relaxation}

Relaxing eases stress and lessens back pain, therefore allow yourself time to relax.

It's your back, it's your choice, but remember, you can ease your pain! 


\section{Tell us what you think !}

\section{After reading "Back Home"}

please tell us what you think about the information.

Your answers and comments help us to know how we can give out information that helps people to learn.

For each statement, mark your answer with a tick.

There is a place to add your comments on the back of the page.

I found the words and sentences:

1 too simple

$\frac{1}{9}$ just right

o too difficult

I found the print size:

5 too small

5 just right

o too large

I found the page size:

3 too small

7 just right

o too big

I found the colours:

— made the words easy to read made the words hard to read

I found the pictures:

5 helped me to learn about the information

I did not help me to learn about the information 
I got this information:

$\frac{0}{1}$ too soon
$\frac{1}{q}$ too late

I found the information:

1 too simple

9 just right

o too difficult

to understand

I found the amount of information

2 not enough

8 about right

o too much

I found the information:

5 very helpful

5 a little helpful

not helpful at all

( 1 mussing)

I would have liked more information about:

Posture

Exercusen to otnengtien mys hack

I would have like less information about:

Please write your comments here:

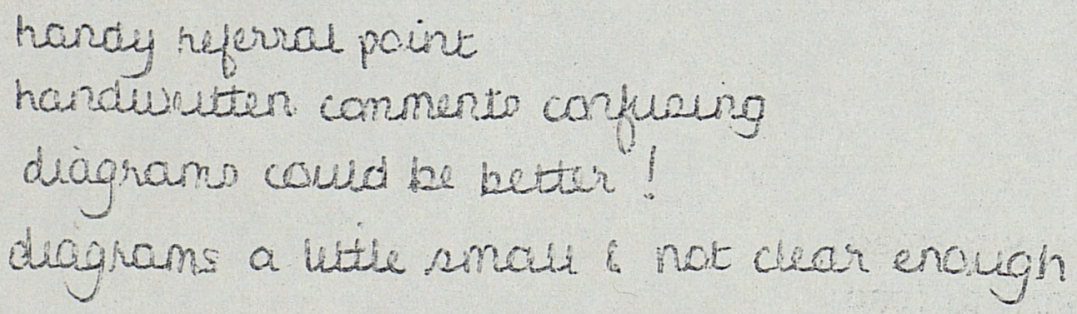

\section{Thank you for your help !}

Please return to: Lisa Smith

Physiotherapy Dept

Southampton General Hospital

Tremona Road

Southampton SO9 $4 \mathrm{XY}$ 


\section{"Back Home" Project}

\section{Consent to take part in a study on the management of patients with acute low back pain at home.}

I consent to participate in the study looking at the effectiveness of a leaflet containing information on the management of acute low back pain at home.

* I agree to complete a questionnaire at the start of the study which explores my approach to the management of patients with acute low back pain

* If I am in the leaflet group of the study, I will strongly support the use of the leaflet

* I agree not to use any other leaflets on back pain for the duration of the study

* $\quad$ Otherwise, I agree not to change the advice that I give to patients with acute low back pain for the duration of the study ( $+/-12$ months).

* I will identify appropriate patients with acute low back pain and get their written consent to participate in this study.

* I will notify you if the patient's treatment plan changes. eg. Referrals elsewhere

* I am prepared to record any questions that patients ask when contacting the Practice during the trial.

* I will complete an end of study questionnaire.

I know that I can stop being in this study at any time without consequence.

I know that all information collected in this study will be strictly confidential.

NAME:

SIGNATURE

DATE: 


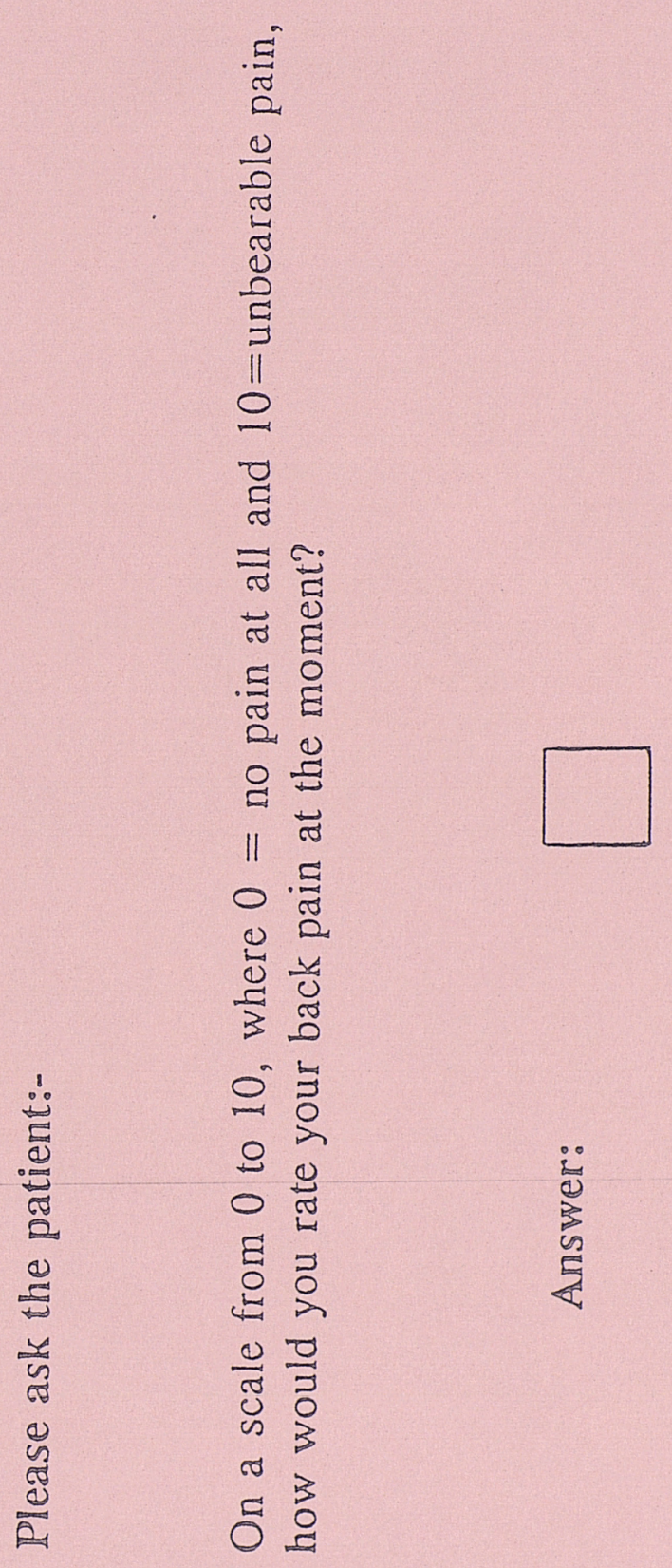


GP's Name:

\section{"BACK HOME" PROJECT}

\section{PROCEDURES FOR INCLUDING A PATIENT IN THE ACUTE LOW BACK PAIN STUDY}

When you are contacted/consulted by a person with acute low back pain, bad enough to require time off work:-

1)

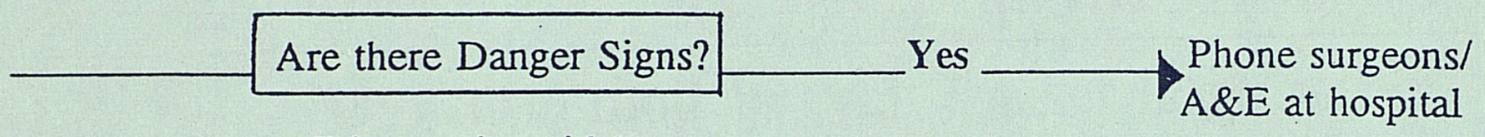

2)

(Diagnostic guide)

A\&E at hospital

3)

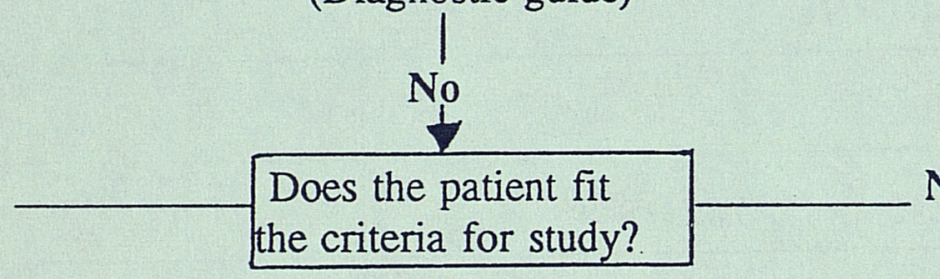

No Excluded from Study

(List of criteria)

Yes

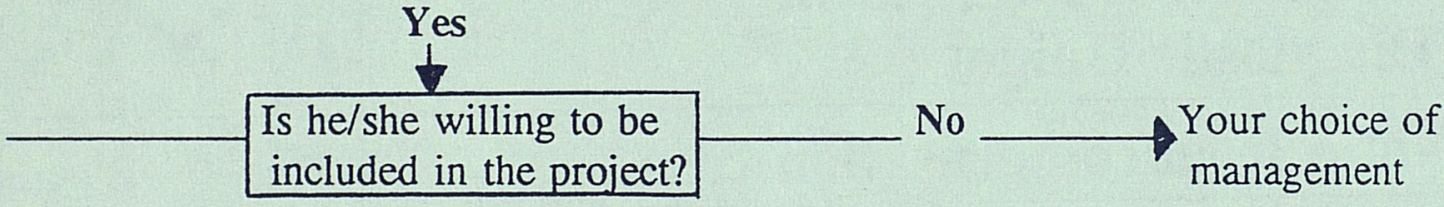

(Consent form)

4)

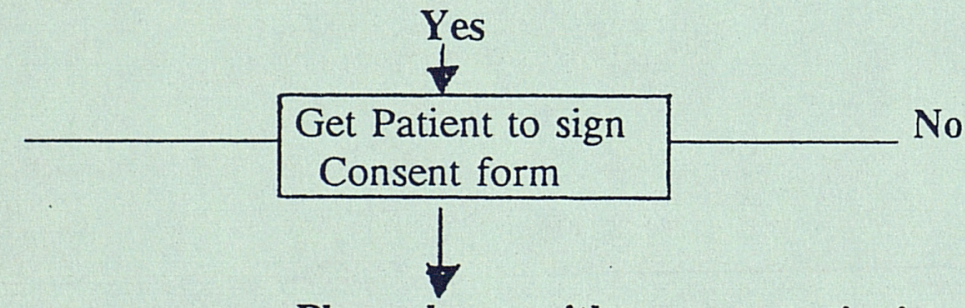

No Your choice of management

5)

Please leave with your receptionist:-

a) Patient's signed consent form

b) Your signature on Criteria Form $\begin{aligned} & \text { + ALLOTHER } \\ & \text { PAPERWORK! }\end{aligned}$

6) Please telephone the project ansaphone:-

(0703) 794564

\& record the patient's name, address and telephone number 


\begin{tabular}{|c|c|}
\hline $\begin{array}{l}\text { CRITERIA FOR INCLUSION IN } \\
\text { ACUTE LOW BACK PAIN STUDY }\end{array}$ & $\begin{array}{r}\text { TICK } \\
\text { IF YES }\end{array}$ \\
\hline \multicolumn{2}{|l|}{ 16-60 Age Group } \\
\hline \multicolumn{2}{|l|}{$\begin{array}{l}\text { Is taking at least } 3 \text { days at home (off } \\
\text { work) or works at home }\end{array}$} \\
\hline \multicolumn{2}{|l|}{ Willing to take part } \\
\hline \multicolumn{2}{|l|}{ No back pain in past 6 months } \\
\hline \multicolumn{2}{|l|}{ Not attended Back School } \\
\hline \multicolumn{2}{|l|}{$\begin{array}{l}\text { Danger signs not present } \\
\text { (Diagnostic Guide) }\end{array}$} \\
\hline \multicolumn{2}{|l|}{ Not a legal/compensation case } \\
\hline $\begin{array}{l}\text { Name of Patient: } \\
\text { Address: }\end{array}$ & ID: \\
\hline \multicolumn{2}{|l|}{ Phone No: } \\
\hline \multicolumn{2}{|l|}{ G.P. Signature: } \\
\hline G.P. Phone No: & \\
\hline
\end{tabular}

\footnotetext{
NB NO TREATMENT (ie. CHIRO/OSTEO/PHYSIO) FCR THIS EPISODE
} 
5.7 Inclusion criteria 


\section{"Back Home" Project}

\section{Consent to take part in a study on the management of people with acute low back pain at home}

I agree to join this study. This study is looking at two different ways of taking care of people with acute low back pain.

I have talked to my GP about what happens in this study. I understand that:

In the first week of the study

* A Research Assistant visits me in my home to ask me questions about my low back pain.

* I fill in some questionnaires.

This happens again 2-3 weeks later and after 3 months

I know that I will receive the usual treatment from my GP.

I also know that I can stop being in the study at any time that I choose. This will, in no way, affect the care that I receive from my GP's Practice.

I know that the answers that I give to the questions are kept in confidence. That means that my name does not appear on the questionnaires and is only seen by the staff working on this study. My name will not be recognisable in any reports or presentations done on this study.

My signature here means that all these things have been explained to me and I agree to take part in the study.

Name:

Signature:

Witness:

Date: 


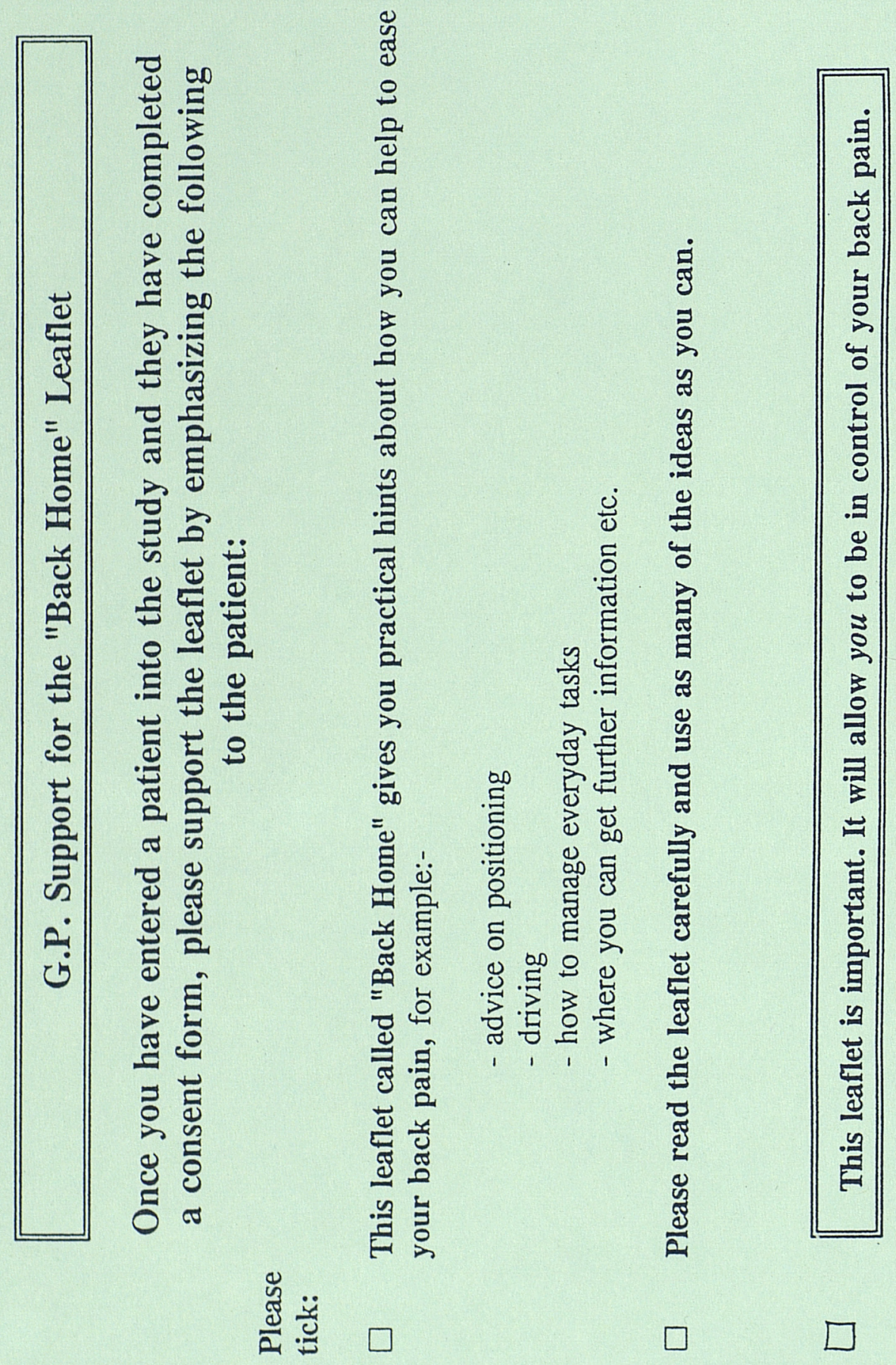


I.D. No.

Assessment

Date

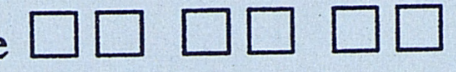

\section{"Back Home" Questionnaire}

Please select one answer only to each of the following questions. Choose the answer which you think is the best and circle the letter as shown: (b)

1) What do you think is the commonest cause of back pain?
(a) arthritis
(b) longstanding bad posture
(c) becoming overweight
(d) sudden accident or injury
(e) a slipped disc
(f) don't know

2) Which position puts least stress on your back?

(a)

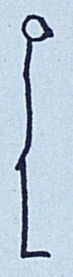

(b)

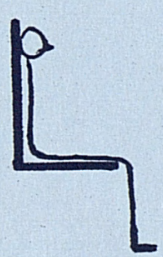

(c)

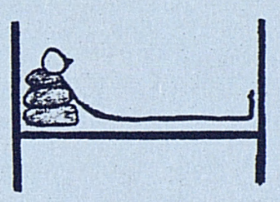

(d)

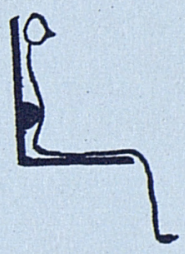

(e)

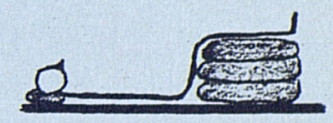

3) Which of these is most likely to make your pain worse?
(a) walking about
(b) leaning against a wall
(c) sitting in a soft chair
(d) lying on a firm bed
(e) lying on your front
(f) don't know

4) What is the easiest position in which to put your socks/tights on, when your back is really sore?
(a) standing up
(b) sitting down
(c) stooping forward
(d) lying down
(e) kneeling down
(f) don't know 
5) If you apply ice to your back (eg. frozen peas wrapped in a damp towel), how long should you leave it on your skin?
(a) 5 minutes
(b) 10 minutes
(c) 15 minutes
(d) 20 minutes
(e) 30 minutes
(f) don't know

6) If you sit or stand, how frequently should you change your position?
(a) every 5-10 minutes
(b) every 20-30 minutes
(c) every 40-50 minutes
(d) every hour
(e) every 2 hours
(f) don't know

7) The following parts of your back can cause back pain. Applying heat (eg. a hot water bottle or heat pad), can best relieve pain in which one of these?
(a) muscles
(b) nerves
(c) discs
(d) joints
(e) ligaments
(f) don't know

8) How should you sit?

(a)

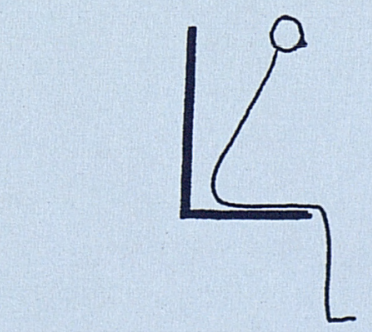

(b)

(c)

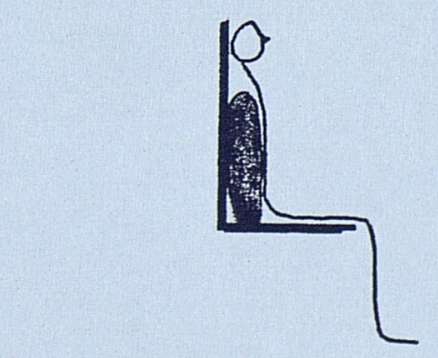

(d)

(e)
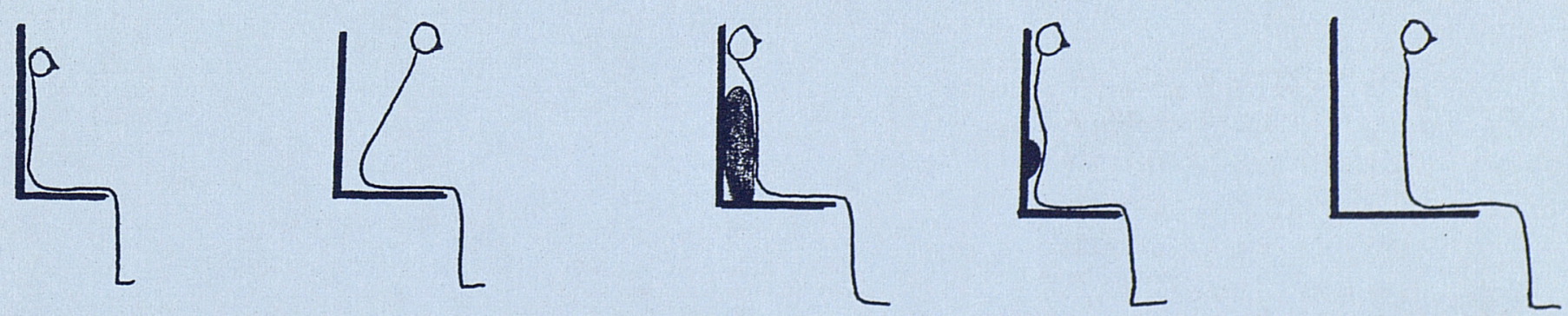

9) What is the best form of exercise once you are up and about?
(a) walking
(b) jogging
(c) running
(d) cycling
(e) dancing
(f) don't know 
I.D. No. $\square \square \square$

Assessment $\square$

Date $\square \square \square \square \square \square$

\section{Attitude (A)}

What do you think caused your back pain?

Please list all the possible factors that may have contributed:-

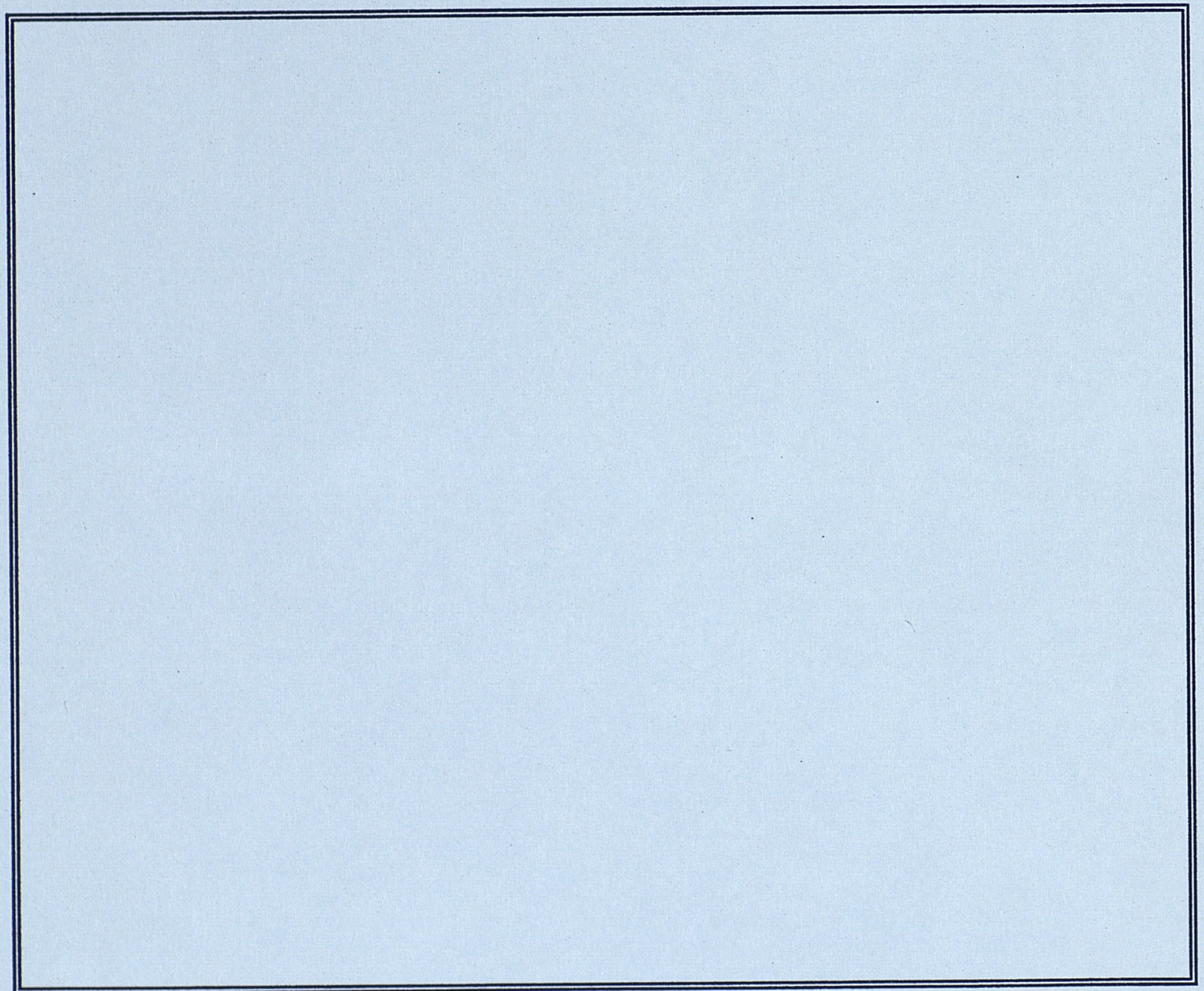


I.D. No. $\square \square \square$

Assessment $\square$

Date

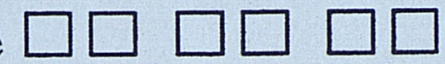

\section{Attitude (B)}

1) Which of the following do you think caused your back pain?

Please tick all that apply eg. $\square$

Random bad luck

Arthritis

A bone or joint "out of place"

Longstanding bad posture

Bad lifting technique

Bad sitting posture

Generally unfit

A bed that is too hard

A bed that is too soft

A sudden accident or injury

Becoming overweight

Too much gardening

Too much sport

Getting out of bed

Getting out of a chair

Picking something up from the floor

Sudden movements eg. coughing

Family history of back trouble

Other (please specify) 
2) Can you reduce your back pain by any of the following?

Please tick all that apply.
a) Tablets
b) Resting in bed
c) Keeping on the move
d) Supporting your back
e) Gentle exercise

3) Do you think you can manage your back pain on your own?

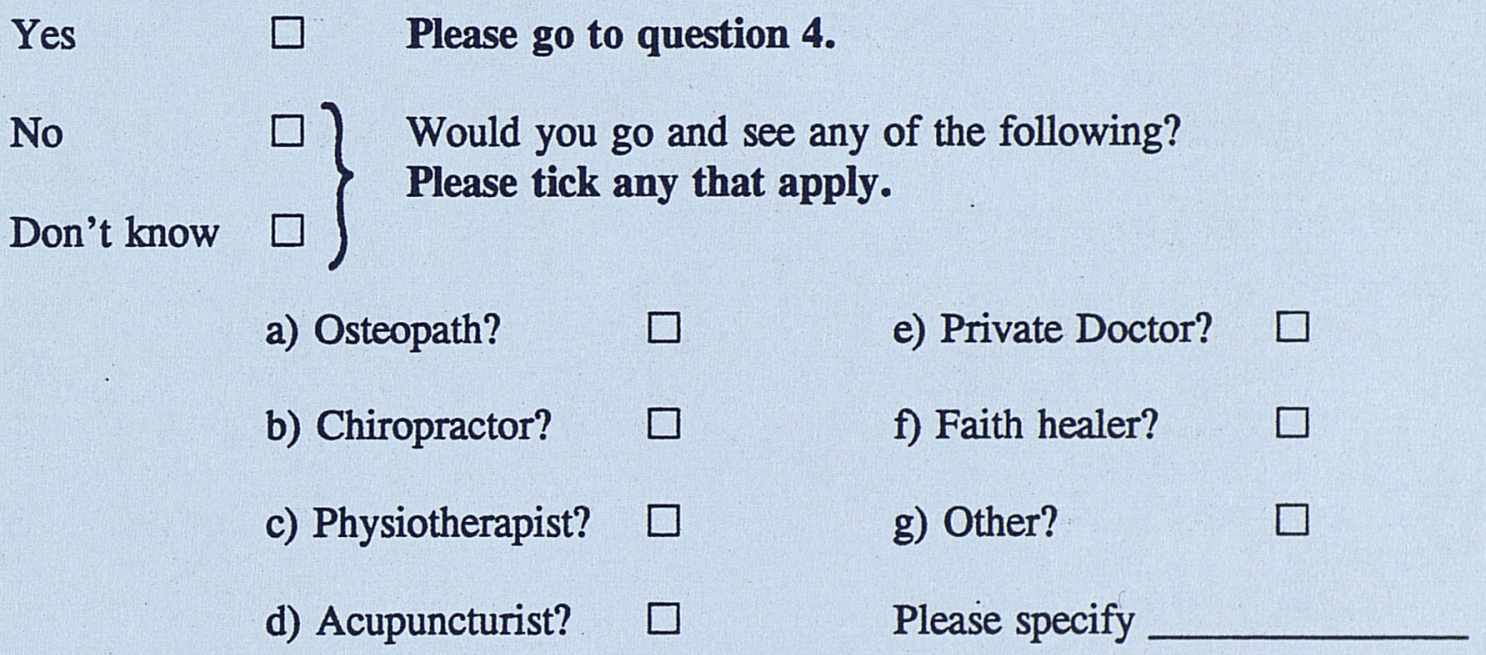

4) Do you feel clear about:-
a) How to control your pain?
Yes $\square$ No $\square$ Don't know
b) How to manage at home?
Yes $\square$ No $\square$ Don't know
c) When to return to work?
Yes $\square$ No $\square$ Don't know
d) How to get fit for work?
Yes $\square$ No $\square$ Don't know
e) How to prevent the pain coming back? Yes $\square$ No $\square$ Don't know

5) Do you think you can prevent your pain coming back again?

Yes $\square \quad$ No $\square \quad$ Don't know $\square$


I.D. No. $\square \square \square$

Assessment $\square$

Date $\square \square \square \square \square \square$

For office use

Total $\square \square \square$

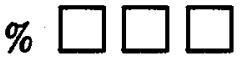

\section{Satisfaction Questionnaire 2}

The line below represents a continuous scale. In the example a person with toothache has marked the line to show that he is reasonably satisfied with the care he received from his dentist:-

Example

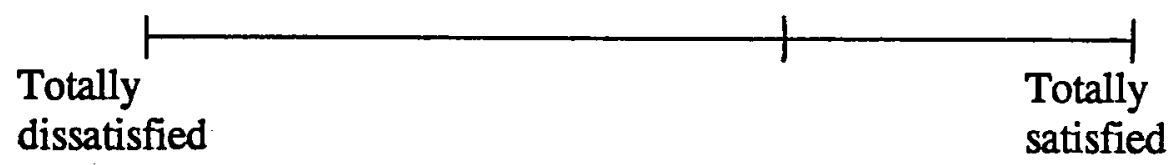

In a similar way please mark the line at the point which shows your level of satisfaction with the following:-

1) How satisfied are you with the amount of information you have received for your back pain?

For office use

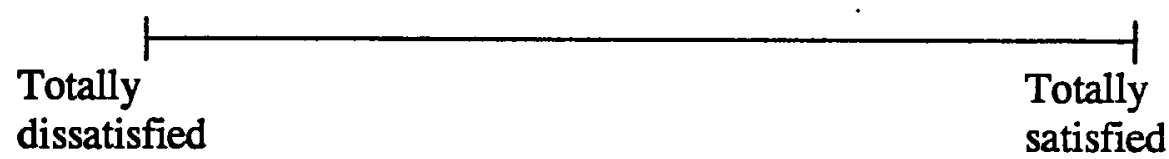

2) How satisfied are you with the content of information you have received for your back pain?

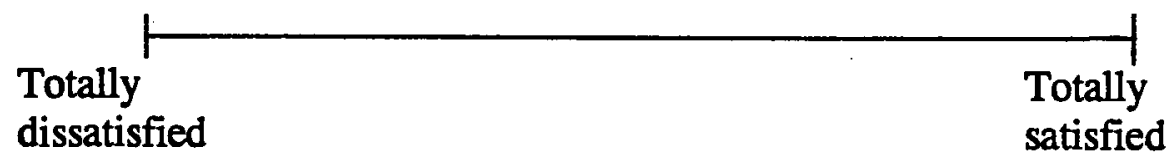

3) How satisfied are you with the way your back pain is being managed?

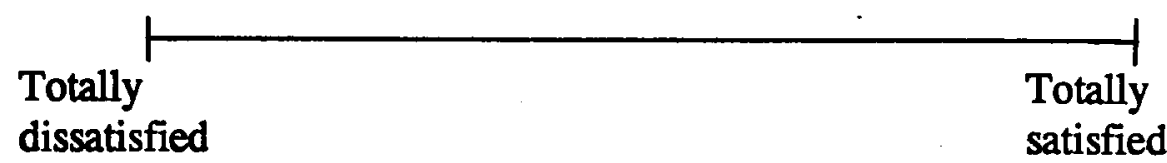


I.D. No. $\square \square \square$

Assessment $\square$

Date $\square \square \square \square \square \square$

\section{Observable Behaviour Checklist}

\section{Activity 1}

Getting up from lying down.

1) Did they roll onto their side first?

\section{Activity 2}

Sitting down (eg. during the interview).

2) Did they attempt to support the lumbar lordosis

Yes No (eg. with a pillow, cushion etc.)?

\section{Activity 3}

Getting up from sitting down (eg. at the end of the interview).

3) Did they move to the front of the chair first?

\section{Activity 4}

Picking a light object up from the floor.

4) Did they attempt to bend their knees?

5) Did they have a suitably wide base of support? (ie. feet apart $>$ or $=$ to distance of the shoulders). 


\section{- Vanderbilt UniversityMedical Center}

School of Nursing

Health Care Research Project

Direct Phone: (615) 322-2520

E-mail: WALLSTKA@CTRVAX.VANDERBILT.EDU
Godchaux Hall

Nashville. TN 37240-0008

Telephone (615) $322-4400$ FAX (615) 343-7711

To:

Fellow Health Researcher

From:

Kenneth A. Wallston, Ph.D. KAW

Re:

The Multidimensional Health Locus of Control (MHLC) Scales

Thank you for your recent inquiry about our MHLC scales. Enclosed you will find copies of all three forms of the MHLC (Forms A, B, \& C) along with scoring instructions for the forms.

Forms A \& B are the "general" health locus of control scales that have been in use since the midlate 1970's (and were first described in Wallston, Wallston, \& DeVellis, 1978, Health Education Monographs, $6,160-170$.) Each of these two "equivalent" forms contain three 6 item subscales: internality; powerful others externality; and chance externality. In the past $15+$ years, forms A/B have been used in nearly a thousand studies and have been cited in the literature hundreds of times.

Form $C$ is a relatively new version of the scale that we first started to develop in 1987 . Form $\mathrm{C}$ is designed to be "condition-specific" and can be used in place of Form A/B when studying people with an existing health/medical condition. [The way you make this happen is to replace the word "condition" in each item with whatever condition (e.g., arthritis, diabetes, pain, etc.) your subjects have.] Form C has not yet been published, but sufficient work has been done with it that we are confident it is in its final usable form. Like Forms A/B, Form C also has 18 items, but, instead of a single 6 item powerful others subscale, Form $\mathrm{C}$ has two, independent 3 item subscales: doctors, and other people.

We consider all three forms of the MHLC to be "in the public domain." That means that you are free to use the scales in your research (and to alter them for your research in any way you choose) without obtaining our explicit permission. We do ask, however, that you cite the scales correctly if/when you use them ${ }^{1}$. If you profit monetarily from the use of our scales, we expect that a suitable contribution would be made to "The Vanderbilt Health Care Research Project." If you are a student, you have our permission to include a copy of our scale(s) in the appendix to your thesis or dissertation; otherwise, it would be unethical to publish these scales without obtaining our explicit written permission to do so.

I have recently written and copyrighted a manual for the use of the MHLC scales. It is not necessary for you to purchase a copy of this manual in order to use the scales, but, if you would like to purchase a copy, please send a check for $\$ 10.00$ (US) made out to "Vanderbilt University" to: Health Care Research Project; School of Nursing; Vanderbilt University Medical Center; Nashville, TN 37240.

1 If you need/want to cite Form C, you may use the following citation:

Wallston, K.A., Stein, M.J., \& Smith, C.A. (1993). Form C of the MHLC Scales: A conditionspecific health locus of control scale. Unpublished manuscript. Vanderbilt University. Nashville, TN. 
RUCTIONS: Each item below is a belief statement about condition with which you may agree lisagree. Beside each statement is a scale which ranges from strongly disagree (1) to , Jngly agree (6). For each item we would like you to circle the number that represents the ent to which you disagree or agree with the statement. The more strongly you agree with a jament, then the lower will be the number you circle. Please make sure that you answer ry item and that you circle only one number per item. This is a measure of your personal isefs; obviously; there are no right or wrong answers.

\section{1=STRONGLY DISAGREE 2=MODERATELY DISAGREE $3=$ SLIGHTLY DISAGREE}

If my condition worsens, it is my own behavior which determines how soon I feel better again.

As to my condition, what will be will be.

If I see my doctor regularly, I am less likely to have problems with my condition.

Most things that affect my condition happen to me by chance.

Whenever my condition worsens, I should consult a medically trained professional.

If my condition worsens, it is my own behavior which determines how soon I feel better again.

other people play a big role in whether my condition improves, stays the same, or gets worse.

Whatever goes wrong with my condition is my own fault.

Luck plays a big part in determining how my condition improves.

In order for my condition to improve, it is up to other people to see that the right things happen.

Whatever improvement occurs with my condition is largely a matter of good fortune.

The main thing which affects my condition is what I myself do.

I deserve the credit when my condition improves and the blame when it gets worse.

Following doctor's orders to the letter is the best way to keep my condition from getting any worse.

If my condition worsens, it's a matter of fate.

If I am lucky, my condition will get better.

If my condition takes a turn for the worse, it is because I have not been taking proper care of myself.

The type of help I receive from other people determines how soon my condition improves.

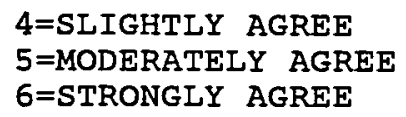

\begin{tabular}{|c|c|c|c|c|c|}
\hline SD & $M D$ & D & A & $M A$ & $S A$ \\
\hline 1 & 2 & 3 & 4 & 5 & 6 \\
\hline 1 & 2 & 3 & 4 & 5 & 6 \\
\hline 1 & 2 & 3 & 4 & 5 & 6 \\
\hline 1 & 2 & 3 & 4 & 5 & 6 \\
\hline 1 & 2 & 3 & 4 & 5 & 6 \\
\hline 1 & 2 & 3 & 4 & 5 & 6 \\
\hline 1 & 2 & 3 & 4 & 5 & 6 \\
\hline 1 & 2 & 3 & 4 & 5 & 6 \\
\hline 1 & 2 & 3 & 4 & 5 & 6 \\
\hline 1 & 2 & 3 & 4 & 5 & 6 \\
\hline 1 & 2 & 3 & 4 & 5 & 6 \\
\hline
\end{tabular}

$\begin{array}{llllll}1 & 2 & 3 & 4 & 5 & 6 \\ 1 & 2 & 3 & 4 & 5 & 6 \\ 1 & 2 & 3 & 4 & 5 & 6\end{array}$

$\begin{array}{llllll}1 & 2 & 3 & 4 & 5 & 6 \\ 1 & 2 & 3 & 4 & 5 & 6\end{array}$




\author{
Physiotherapy Department \\ Level B \\ West Wing
}

31 May 1994

Dr K A Wallston

Director, Healthcare Research Project

School of Nursing

Vanderbilt University Medical Centre

Godchaux Hall

21st Avenue $\mathbf{S}$

Nashville

TN 37240

Dear Dr Wallston

\title{
Re: Multidimensional Health Locus of Control Scales
}

Thank you for forwarding the information I recently requested regarding the Multidimensional Health Locus of Control Scales, which we are using in a back pain study in Southampton, England.

I was particularly interested in the new form $\mathrm{C}$, and would like to know:-

1) the reasons why questions 1 and 6 are identical in form $C$, unlike forms $A \& B$

2) whether you are aware of any studies, or have any data outlining the use of form $\mathrm{C}$ in patients with low back pain?

I look forward to hearing from you, and once again, many thanks for your help and support.

Yours sincerely

\section{Lisa Smith}

Research Physiotherapist

For and on behalf of the "Back Home" Research Team. 


\title{
פVanderbilt University Medical Center
}

School of Nursing

June 11, 1994

Lisa Smith

Physiotherapy Department

Level B, West Wing

Southhampton General Hospital

Tremona Road

Southhampton S09 4XY

ENGLAND

Dear Ms. Smith:

\section{Re: Your letter dated 31 May 1994}

Your letter caused quite a stir back here. You asked why questions 1 and 6 were identical in form C (of our MHLC Scales) when they weren't identical in forms A/B. Well, they weren't supposed to be identical, but the typist made a mistake and typed the same item twice, and none of us caught it. Unfortunately, we've been sending out these incorrect versions of Form $\mathrm{C}$ all over the world since January. You are the first (and, so far, only) person who has pointed out our error, and we are taking steps to correct it immediately. Luckily, we keep a data base of all persons to whom we send basic MHLC packets, so we can at least send them a new form $\mathrm{C}$ (enclosed) and a letter of apology. Thank you for pointing out (politely) our goof.

As for your wanting to know if I am aware of any studies using form $\mathrm{C}$ with low back pain patients, I am afraid that I do not know of any published studies. About four years ago, Don Penzien and his colleagues at the University of Mississippi Medical Center were studying low back pain patients using a pain locus of control scale that was similar to form $\mathrm{C}$, but not quite identical. I do not know what became of that work. Should you wish to get in touch with Dr. Penzien, his address is:

\author{
Department of Psychiatry and Fiuman Behavior \\ University of Mississippi Medial Center \\ 2500 N. State Street \\ Jackson, MS 39216-4505
}

Good luck with your research, and thanks again for pointing out our mistake.

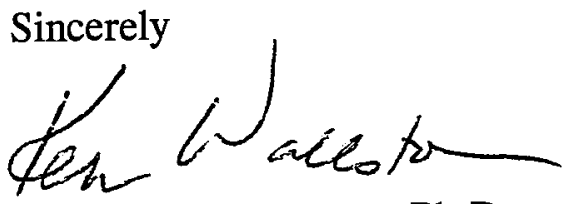

Kenneth A. Wallston, Ph.D.

Professor

Enclosure 


\section{QVanderbilt UniversityMedicalCenter}

Yenneth A. Wallston, Ph.D.

Professor of Psychology in Nursing .

Director, Healthcare Research Project

June, 1994

To:

From:

Whom It May Concern

Re: $\quad$ Form $\mathrm{C}$ of the MHLC Scales

Our records indicate that sometime in the past six months we sent you a packet of information about our Multidimensional Health Locus of Control Scales. Recently, somone pointed out that there was an error in one of the items on the version of Form $\mathrm{C}$ which was included in that packet. On that version, items 1 and 6 were identical. We are, of course, embarrassed for not having caught the typographical mistake, and we hope that it has not caused you any difficulty in your research ${ }^{1}$. A new, corrected version of Form $\mathrm{C}$ is enclosed. Please get rid of the incorrect version and replace it in your files with the corrected version.

The article about the development and initial psychometric properties of Form $\mathrm{C}$ has been accepted for publication in the Journal of Personality Assessment and should come out sometime this year.

${ }^{1}$ If, by some chance, you went ahead and used the incorrect version of Form $\mathrm{C}$ in your research without realizing the mistake, all is not lost. If you have not yet done your data analysis, average the two identical items (\#s 1 and 6). Then add this average to the other four items on the internality subscale (items $8,12,13, \& 17$ ) and multiply the sum by $6 / 5$ ths. [Luckily, all this is easy on a computer!] 
I.D. No. $\square \square \square$

Assessment

Date $\square \square \square \square \square \square$

\section{M.H.L.C. (c)}

Please place a tick in the box $\varangle$ to represent how you feel about each of these statements:-

1) If my back pain worsens, it is my own behaviour which determines how soon I feel better again.

2) As to my back pain, what will be will be.

3) If I see my doctor regularly, I am less likely to have problems with my back pain.

4) Most things that affect my back pain happen to me by chance.

5) Whenever my back pain worsens, I should consult a medically trained professional.

6) I am directly responsible for my back pain getting better or worse.

7) Other people play a big role in whether my back pain improves, stays the same, or gets worse.

8) Whatever goes wrong with my back pain is my own fault.

9) Luck plays a big part in determining how my back pain improves.

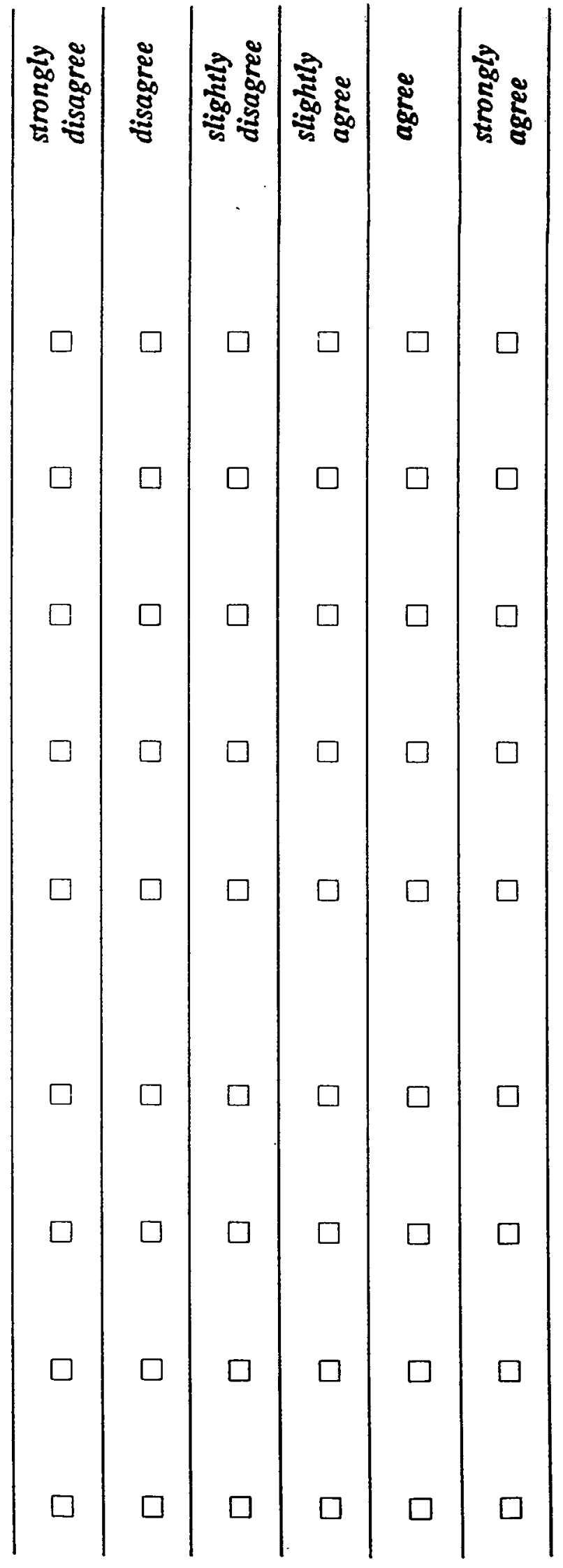


10) In order for my back pain to improve, it is up to other people to see that the right things happen.

11) Whatever improvement occurs with my back pain is largely a matter of good fortune.

12) The main thing which affects my back pain is what I myself do.

13) I deserve the credit when my back pain improves and the blame when it gets worse.

14) Following doctor's orders to the letter is the best way to keep my back pain from getting any worse.

15) If my back pain worsens, it's a matter of fate.

16) If I am lucky, my back pain will get better.

17) If my back pain takes a turn for the worse, it is because I have not been taking proper care of myself.

18) The type of help I receive from other people determines how soon my back pain improves. 


\section{The "Back Home" Study: The Practical Value of a Diagnostic Protocol and Patient Information Leaflet in the Home Management of Back Pain.}

R \& D Project Grant R.52.93.

$$
01.06 .93-31.03 .97
$$

${ }^{1}$ Lisa Smith

${ }^{2}$ Judith Chapman ${ }^{\dagger}$ -

${ }^{3}$ Ted Cantrell ${ }^{\dagger}$

- Superintendent Physiotherapist

${ }^{4}$ Paul Little - GP and Wellcome Training Fellow

${ }^{5}$ Ruth Pickering - $\quad$ Lecturer in Medical Statistics

${ }^{1} J_{0 h n}$ Langridge ${ }^{\dagger} \quad$ - Manager of Physiotherapy Services

${ }^{1}$ Department of Physiotherapy, Southampton University Hospitals Trust (SUHT)

${ }^{2}$ School of Occupational Therapy and Physiotherapy, University of Southampton

${ }^{3}$ Department of Rheumatology and Rehabilitation, SUHT

${ }^{4}$ Primary Care, Faculty of Medicine, University of Southampton

${ }^{5}$ Department of Statistics and Computing, University of Southampton

Correspondence to Lisa Smith

${ }^{\dagger}$ denotes grant holders 

patient Information Leaflet in the Home Management of Back Pain.

Objectives: The hypothesis is that "an optional G.P. diagnostic guide supplemented by a patient advice leaflet improves the ability of people with acute low back pain to manage the episode of pain at home".

Setting: This study was undertaken in primary care where G.Ps recruited 64 patients with acute low back pain. Assessments took place in the patients' home.

Design: The study design was a (blinded) randomised controlled trial. Randomisation occurred at the G.P. practice level into: a) the control group, where patients continued with the G.Ps current management, or b) the experimental (leaflet) group, where patients also received the "Back Home" leaflet. Patients were visited at 2 days, 2 weeks and 3 months, where they completed a total of 15 self-assessment measures. Aspects of their behaviour were discretely recorded by a 'blinded' researcher.

Main outcome measures: Four primary outcomes were identified: knowledge, attitude, behaviour, and function.

Results: One question in the knowledge questionnaire which specifically related to knowledge about sitting posture was significantly different between the two groups: $\mathrm{P}=0.0038$. This change in knowledge was also reflected in a change in behaviour (sitting with the lumbar lordosis supported e.g. with a cushion, pillow etc.), which the patient was unaware they were being observed on. This was significantly different $P=0.0027$. This difference was still reflected at the 3 month assessment. Results show that there was no significant difference in the functional outcome throughout. The leaflet group were also significantly better at maintaining a wide base of support when lifting a light object from the floor, than the control group: $\mathrm{P}=0.0004$.

Discussion: These results suggest that an educational intervention can change knowledge and behaviour in relation to sitting, which has implications for the management of acute low back pain, with potential health gain. The study underlines the importance of clear advice and empowerment at the initial G.P. consultation for patients with acute low back pain. 


\section{Recruitment in the \\ "Back Home" Study}

The recruitment of patients into the "Back Home" study was slower than had been anticipated (64 patients in $2 \frac{1}{2}$ years). We are anxious to follow this up as it has obvious implications for further research studies in General Practice.

Please could you spare a few minutes to complete the following questions and return your answers in the enclosed envelope.

1) Please list any reasons that you consider may have contributed to the slow recruitment into the "Back Home" study:-

2) Are you interested in receiving a copy of the following:
- papers published to date from the Back Home study
Yes No
- a summary of the study findings

If "Yes", please complete:

Your name:

G.P. practice: 
3) To what extent do you think the following factors contributed to the slow recruitment into the "Back Home" study? Please use the scale indicated:

\begin{tabular}{||l|l|}
\hline 1 & $=$ not at all \\
2 & $=$ mildly \\
3 & $=$ moderately \\
4 & $=$ very significantly \\
\hline
\end{tabular}

a) Pressure of work

b) Other research projects also underway in your practice

c) The exclusion criteria prevented the inclusion of many patients

d) G.Ps forgot to include potentially suitable patients

e) It was difficult to find the paperwork

f) The research was hospital-led

g) The research team was multidisciplinary

h) G.Ps had to decide when to enter a patient for the study

i) The process for entering a patient.was too time-consuming

j) The study design (i.e. a randomised controlled trial)

k) Randomisation occurred at the practice rather than the patient level .....

1) G.P.s are over researched

m) Other (please specify)

4) Any other comments.

\section{Thank you for your comments.}

